\title{
Métodos tipo dual simplex para problemas de otimização linear canalizados*
}

Ricardo Silveira Sousa

Orientador: Prof. Dr. Marcos Nereu Arenales

Tese apresentada ao Instituto de Ciências Matemáticas e de Computação - ICMC-USP, como parte dos requisitos para obtenção do título de Doutor em Ciências - Ciências de Computação e Matemática Computacional.

\section{"VERSÃO REVISADA APÓS A DEFESA"}

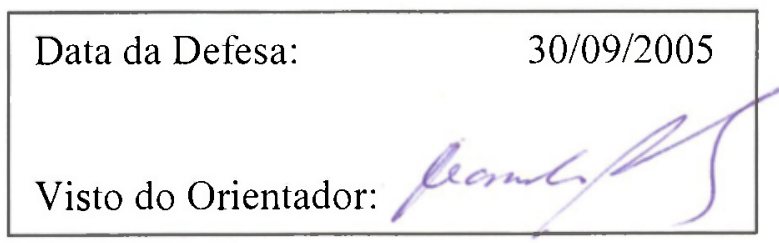

USP - São Carlos

Outubro/2005

*Este trabalho teve o apoio da FAPESP. 
Aos meas queridos pais

Joro Geralde de Sollä Mariar de Lourdes Silveira e co meu irmão Rodrigo em gratidão pelo extensivo apoio no conquistar noves desafios... 


\section{Agradecimento}

Mencionar todas as pessoas que me apoiaram na realização desta tese seria um trabalho impossivel, todavia, desejo expressar minha sincera e profunda gratidão agradecendo:

Ao meu orientador Marcos Nereu Arenales pela suas valiosas orientações, amizade, paciência e incentivo permanente, que me conduziram na realizaçăo desta tese durante os varios anos de pesyulisa.

A Maria Luiza R. Gomes a minha doce filha Yara Comes Sousa pelo grande amor, carinho e incentivo durante todo o desenvolvimento deste trabalho, assim como seus familiares, em especial à Maria Mazaarello.

Ao Prot. Aurélio, a Carla e aos amigos Cássio e Silvio por suas grandes contribuições.

Aos colegas de trabalho: Araxá, Maristela Santos, Marco Lopes, Anderson, Profa. Fran. Kelly, Adriana, Ired, Josemar, Viviane, Sônia, João (jóis, Adriano, Sandra, Lilian, Katliana, Marcelo Cad.

Aos lendários amigos de todos os tempos: Moreno, Beiço, Pio Redondo. Thiago (jabiru, Barra, Bob Lsponja. Werley, Bibi, Thiago Rhampone, Juliano e ao Fernando Perey com grandes momentos de descontração.

$\Lambda$ todos meus amigos de Muriaé em especial: Rodrigo, Thiago, Pepeu. Toninho e seus familiares.

A LAPLSP pelo grande apoio financeiro.

Professores e luncionários do ICMC - USP pelo convívio diário. amizade e respeito.

Principalmente a Deus, por estar ao meu lado a todo instante. 


\section{Resumo}

A otimização linear tem sido objeto de intenso estudo desde a publicaçào do método simplex de Dantzig em 1947. sendo revigorada a partir de $1984 \mathrm{com}$ a publicação de un método de pontos interiores por Karmarkar, o qual demonstrou ser computacionalmente eficiente e com propriedade de convergência polinomial no estudo do pior caso. Embora muitas variantes do málodo simplex não tenham complexidade polinomial, elas apresentam um comportamento polinomial em termos do número de restrições do problema, para inúmeros problemas práticos, constituindo o chamado "lolelore" simplex. Nos últimos anos, tem creseido o interesse pela pescyusa sobre eliciência dos métodos lipo simplex. Há uma pergunta subjacente, que talve» constitua o maior desafio da atualidade na teoria da otimizaçăo linear: "ĺ possível construir um algorimo tipo simplex com complexidade polinomial? Além disso, eficiente do ponto de vista prático?" A resposta a esta pergunta não deve ser trivial e talvez seja negativa. restando por enquanto a tarefa árdua da investigaçào da complexidade, caso a caso, dos métodos propostos. Xeste trabalho aprolindamos a investigação sobre a versão mais utilizada dos métodos do tipo simplex: o método dual simplex, especializado para a forma geral (restrições canalizadas), cujo problema dual é lincar por partes. $A$ importância da forma geral não somente porque as demais formats sâo lacilmente representadats nela, mas porque muitos problemas práticos surgem naturatmente desta maneira e técnicas de pré-processamento que buscam apertar limitantes levam a ela. Foram investigadas buscas unidimensional lineares por partes, como regras anti-ciclagem influenciam positivamente sobre o efeito 'estagnação' decorrente de soluções degeneradas, a regra de Jantyign nomalizada e algumas técnicas de resolução de sistemas lineares. incluindo o método (k) gradiente bi-conjugado. que alimenta grande expectativa no aumento da cticiência computacional para resolução de problemas de grande porle.

Palav ras-chave: Otimização Lincar, Métodos lipo Dual Simplex. Sistemals Lineares Esparsos. 


\section{Abstract}

Linear optmization has been studied in-depth since 1947, when George Dantizg published the Simplex method. From 1984. research on linear optmization was enormously intensified because of the publication of the lnterior Point method by Narendra Karmarkar. which showed to be computationally cificient for many large and sparse problems, and with polynomial time complexity. Although many variants of the Simplex method do not have polynomial time complexity, they perform well for a number of practical problems, presenting polynomial behaviour, and this constitutes a folklore of Simplex type methods. Recently, the interest in the efliciency of Simplex methods has increased. There is an underlying question, which is perhaps the biggest challenge nowadays in the Optimization theory: "Is it possible to build a time polynomial simplex type algorithm, which is efficient from the computational point of vicw?" The answer to this culuestion is certainly not trivial, and probably it is negative. One is faced with the hard task of investigating the complexity, one by one, of the proposed methods. In this work, we study the most used version of the simplex method: the Dual Simplex method. specialized for the general form (two sided constraints), whose dual is a linear piecewise optimization problem. The importance of the general form is not only because it can easily deal with other forms, but many important practical problems arise naturally in this way, and pre-processing techniques of tighting bounds lead to this form. The following aspects were investigated: different piecewise linear line scarching, the effect of stalling phenomena due to degenerate solutions and how anti-cyclic rules can influence it positively, the steepest edge rule, and some techniques to handle imbedded linear systems, including the iterative bi-conjugated gradient method, in which an expectation arises to improve the computational performance for very large and sparse problems.

Keywords: Linear Optimization. Simplex Type methods. Sparse linear systems. 


\section{Sumário}

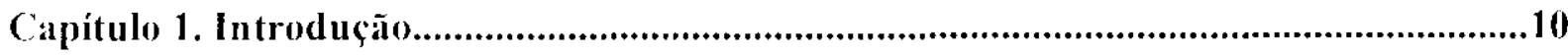

1.1. () Problema de Otimização Linear Canalizado ............................................................11

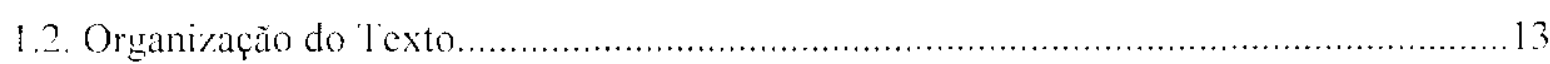

Capítulo 2. Otimização Linear: História, Métodos Tipo Simplex e de Pontos

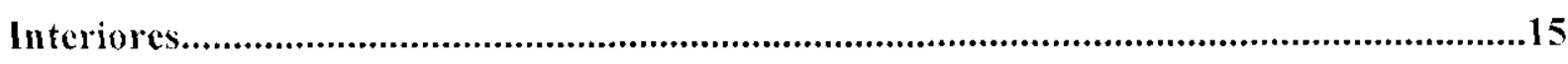

2.1. $1 \mathrm{~m}$ Breve llistórico de Otimizaçầ I.inear .................................................................... 15

2.2. A E.ficiència e a Complexidade Computacional do Método Simplex ............................ 18

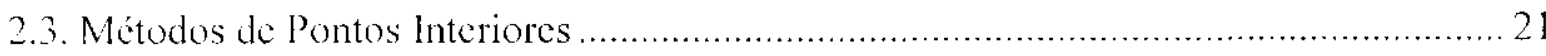

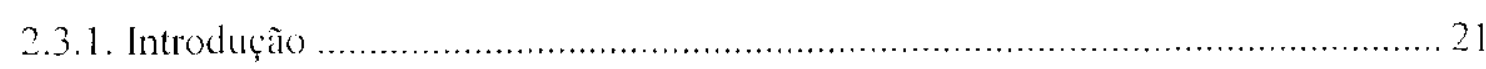

2.3.2. Métodos de Pontos Interiores...................................................................... 22

Capítulo 3. Problemas de Otimização Linear Canalizado.......................................................27

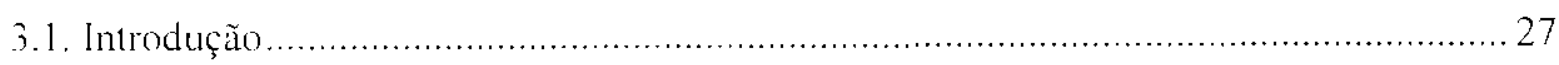

3.2. O Problema de Otimização Linear Canalizado ...................................................... 27

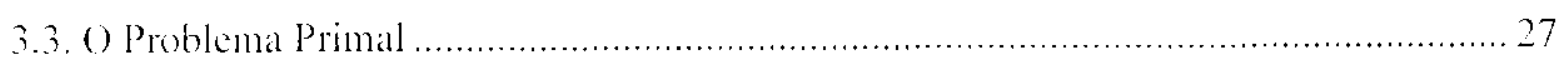

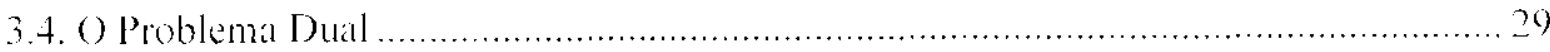


3.5. A Estratégia Dual Simplex

3.6. Algoritmo Dual Simplex com Busca Unidimensional (DSBI I)..................................

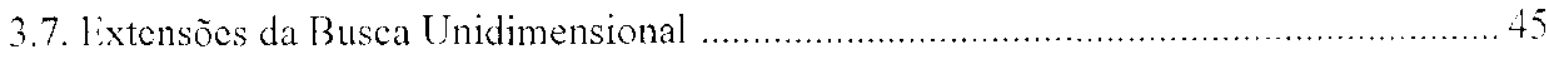

3.7.1. Primeira Modificação no Procedimento de Busca - Busca Parcial (BP))...........to

3.7.2. Segunda Modificação no Procedimento de Busca - Busca Completa (BC _ 2)...46

3.7.3. Um Método de Busca Unidimensional Inexata - Regra de Armijo $(\Lambda R) \ldots \ldots \ldots .47$

Capítulo 4. Técnicas de Esparsidade para o Método Simplex ............................................. 49

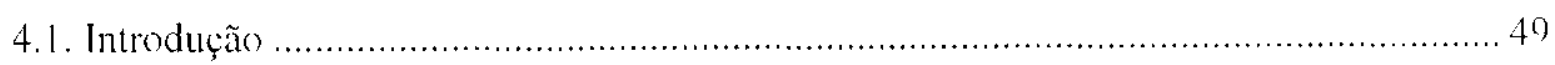

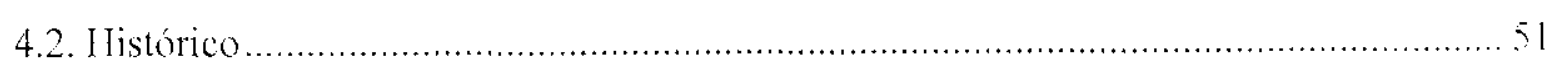

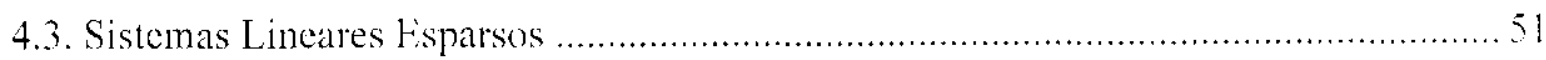

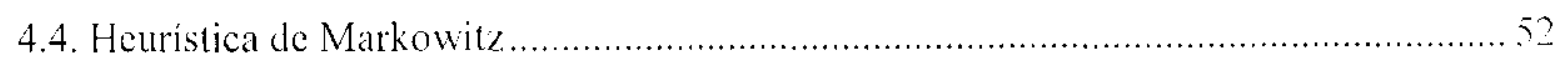

4.5. Heuristica de Grau Mínimo................................................................................... 54

4.6. Método de Atualização de Bartels e Golub ...............................................................54

4.7. Método de Atualização de lorrest e T'omlin .............................................................. 56

4.8. Método de Atualização de Reid .............................................................................. 58

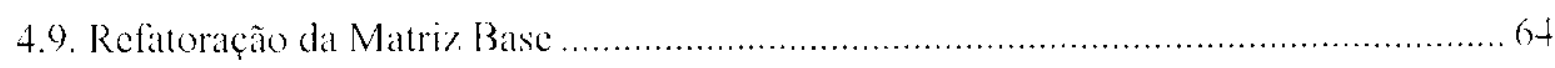

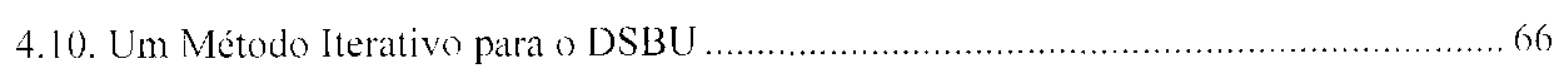

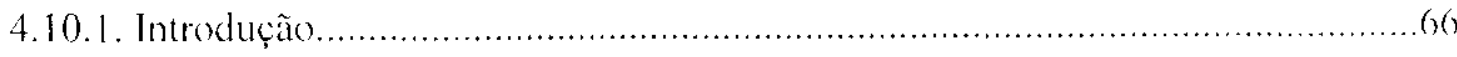

4.10.2. Método do Gradiente Bi-Conjugado ((GBC) ...........................................67

Capítulo 5. Ciclagem, Degeneração e Variantes do Método Simplex.................................. 70

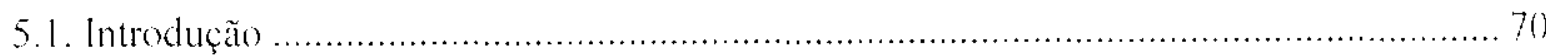

5.2. Ciclagem em Problemas de Otimização Linear .......................................................... 71

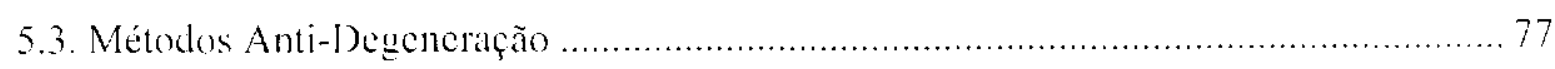

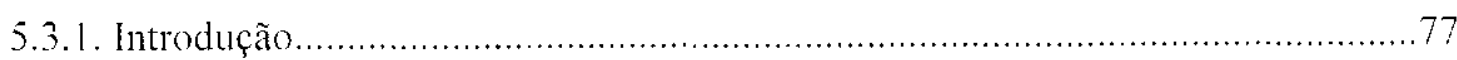

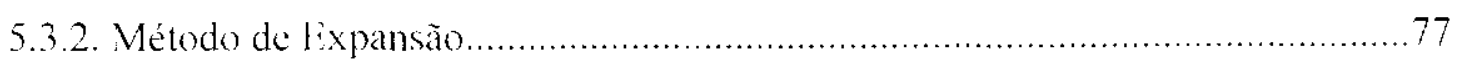

5.3.3. O Método de Perturbação..............................................................................

5.4. Breve Revisão de Variantes do Método Simplex ......................................................

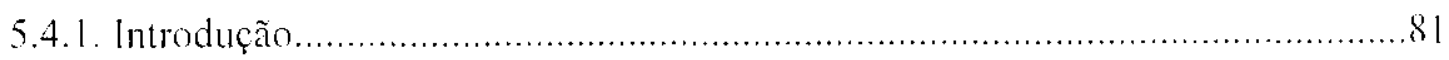

5.4.2. Considerações Sobre Métodos Tipo Simplex..................................................

5.4.3. Métodos Primal-Dual Exterior (Criss-cross) ................................................. 8.

5.4.4. Comentários Sobre Outras Variantes Simplex...............................................84 
Capítulo 6. Esparsidade e Estrutura de Dados

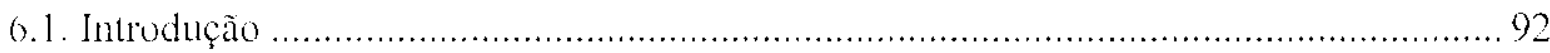

6.2. Estrutura de Dados sugerida por Reid (1982) ........................................... 93

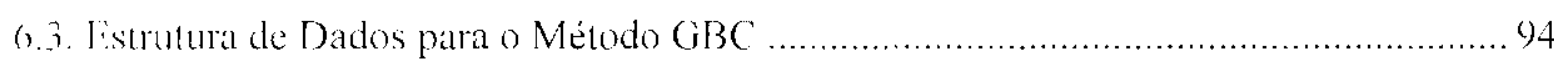

6.4. Lima Estrutura de Dados para o Método Simplex.................................................. 96

6.5. Considerações na Implementação ....................................................... 98

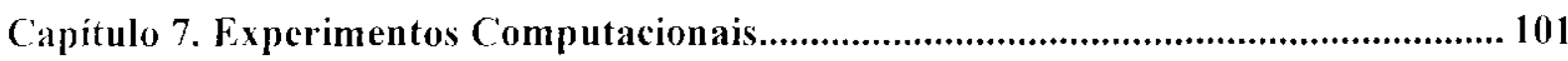

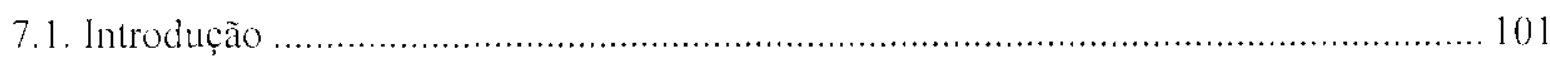

7.2. I leurísticas e Métodos de Atualização da Base .................................................. 101

7.3. Lxtensões na Busca Unidimensional .......................................................... 107

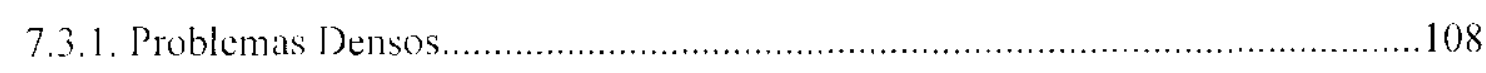

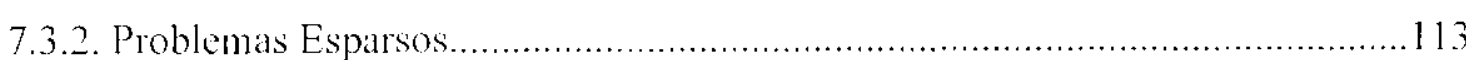

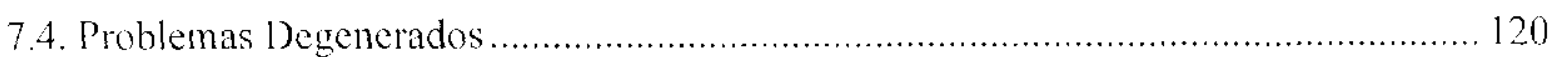

7.5. O Método DSBU com Regra de Dantzig Normalizada ...................................... 124

7.6. O Método DSBU com o Gradiente Bi-Conjugado ............................................. 130

Capitulo 8. Conclusões e Trabalhos Futuros .................................................................... 133

Referências Bibliográficas........................................................................................136

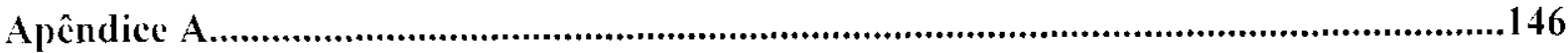

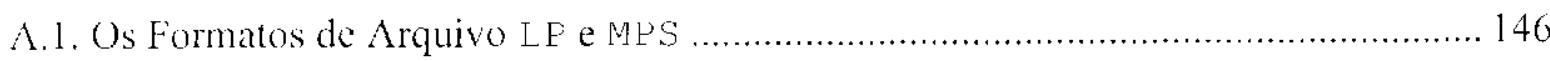

A.2. Hog CPLLX Optimizer e XPRESS Optimizer................................................. 152

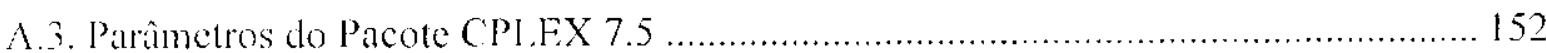

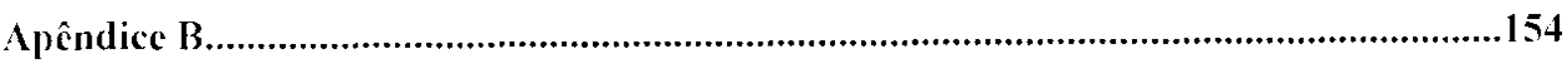

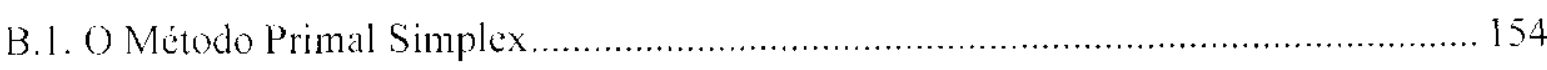

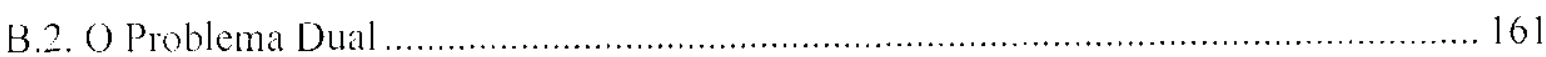

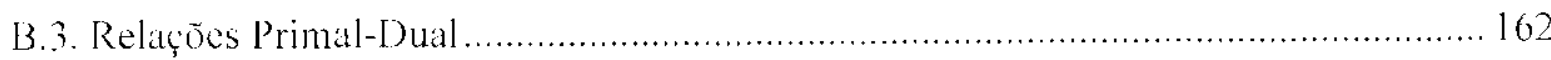




\section{Lista de Figuras}

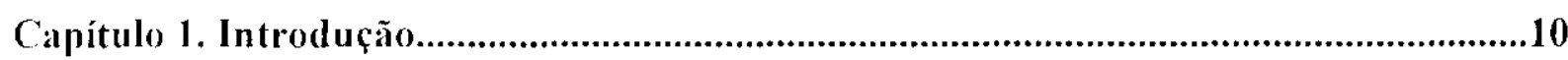

Capítulo 2. Otimização Linear: História, Métodos Tipo Simplex e de Pontos

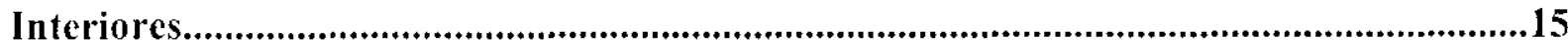

Figura 2.1 - Fxumplar de Klee e Minty com très variáveis................................................17

Figura 2.2 -.. Desempenho médio do método simplex aplicado ao problema de corte...........19

Capítulo 3. Problemas de otimização linear canalizado..........................................................27

Figura 3.1 - Função objetivo dual linear por partes........................................................... 31

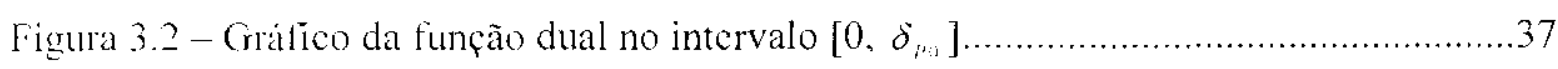

Figura 3.3 - Gráfico da função dual após a mudança de sinal de uma variável básica.........40

Hig̣ura 3.4 - Grálico da função dual: $\delta_{\mathrm{p} 2}$ é o ponto de máximo............................................40

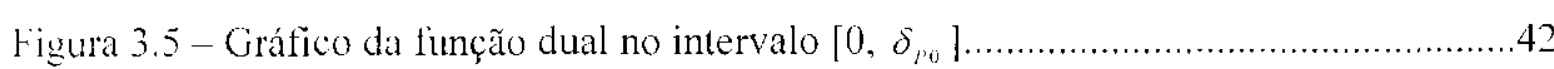

Figura 3.6 - Regra de Armijo para a função dual linear por partes......................................48

Capitulo 4. Téenicas de Esparsidade para o Método Simplex...........................................49

Figura 4.1 - Permutação que preserva a esparsidade na decomposição LU ..........................52 
Figura 4.2 - Matriz triangular superior com uma coluna espeto, em destaque.....................55

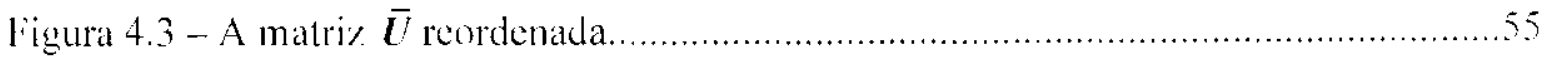

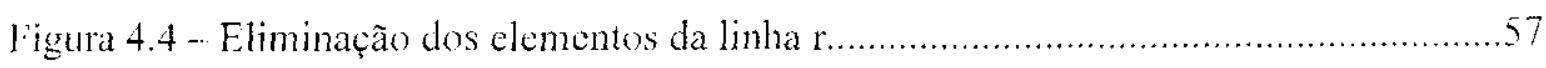

Figura 4.5 - Comparação dos métodos: Bartels \& Golub $\times$ Forrest \& Tomlin..................... 58

Figura 4.6 - A submatriz (pontilhada) no método de Forrest e Tomlin...............................5)

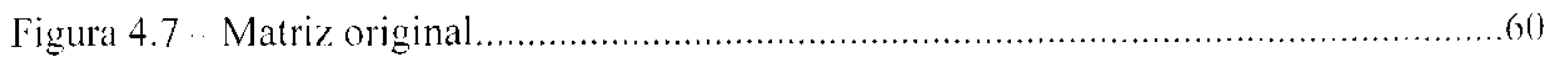

Higura 4.8 - Coluna com único elemento movida para frente da submatriz.......................61

Fïgura 4.9 - I inha com único elemento movida para abaixo da submatriz.......................62

Figura 4.10 - Linha com único elemento movida para abaixo da submatriz.......................6?

ligura 4.11 -- Permutações que resultaram na Hessenberg superior....................................6.3

Figura 4.12 - Coluna com único elemento movida para frente da submatriz.....................63

Figura 4.13 - Coluna com único elemento movida para frente da submatriz........................64

Capítulo 5. Ciclagem, Degeneração e Variantes do Método Simplex..................................70

Capítulo 6. Esparsidade e Estrutura de Dados.................................................................92

Figura 6.1 - Representação da matrił acessando os elementos por coluna..........................94

Figura 6.2 - A matriz $\Lambda 1$ representada pelos vetores ija e sa....................................... 96

Fïgura 6.3 - A matriz $\Lambda 2$ representada por uma coleção de vetores coluna.........................97

Fïgura 6.4 - Representação da matriz na forma geral.......................................................99

Capítulo 7. Experimentos Computacionais....................................................................101

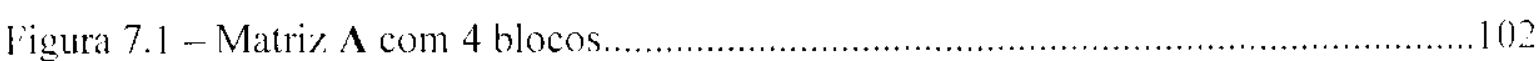

Figura 7.2 - Tempo de Resolução - Problemas Densos....................................................113

Figura 7.3 - Número de Iterações - Problemas Esparsos 20 Blocos.................................128

Capítulo 8. Conclusões e Trabalhos Futuros..............................................................133

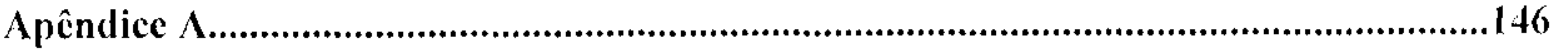

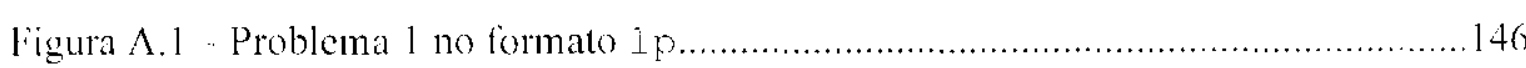

Figura A.2 - O problema 1 no formato mos................................................................... 147

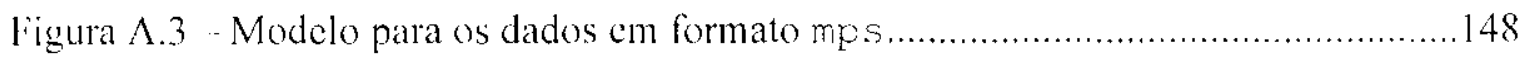

Figura A.4 - A restrição restl representada de duas maneiras........................................150 


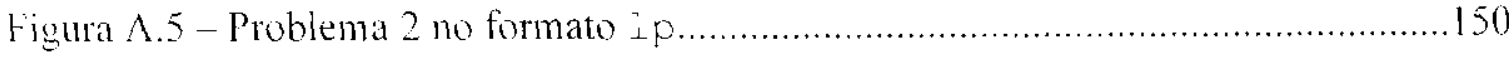

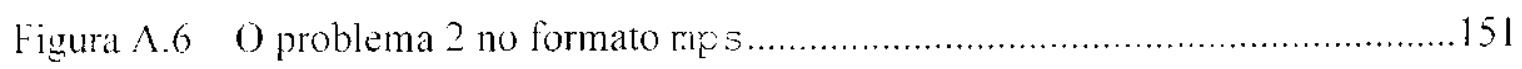

Apêndice B.... 


\section{Lista de Tabelas}

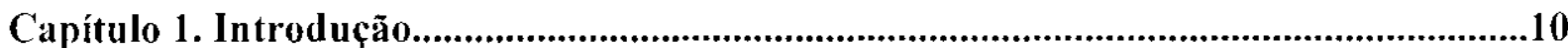

Capítulo 2. Otimização Linear: História, Métodos Tipo Simplex e de Pontos

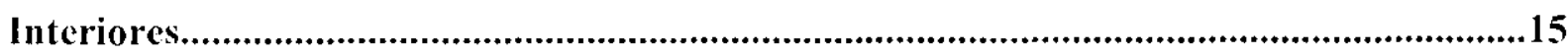

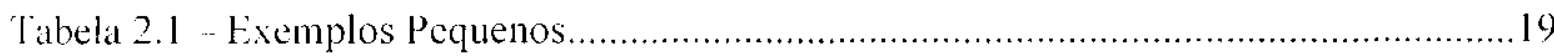

Tabela 2.2 Exemplos Grandes....................................................................... 19

Capítulo 3. Problemas de otimização linear canalizado..................................................27

Capítulo 4. Técnicas de Esparsidade para o Método Simplex..........................................49

Capítulo 5. Ciclagem, Degeneração e Variantes do Método Simplex...............................70

Tabela 5.1 - Experimentos computacionais de problemas com ciclagem..........................76

Capítulo 6. Esparsidade e Estrutura de Dados.............................................................92

Capítulo 7. Experimentos Computacionais.................................................................101 
Tabcla 7.1 - Comparação entre o tempo de resolução.

Tabela 7.2 - Comparação entre alguns valores para o número de tualizações....................104

Tabela 7.3 - Tempo total de resolução de problemas usando gerador escada .....................105

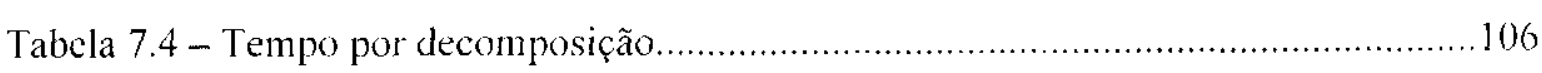

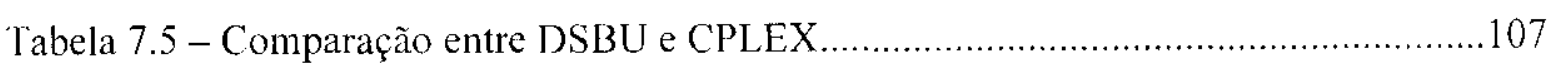

Tabela 7.6 … Busca Completa_l $\times$ Sem Busca - Problcmas Densos.................................109

Tabela 7.7 - Busca Completa_l $\times$ Sem Busca - Problemas Densos.................................... 109

Tabela 7.8 - Busca Completa_l $\times$ Regra de Armijo - Problemas Densos.........................110

Tabcla 7.9 - Tempo Total $\times$ Tempo de Ordenação..........................................................110

Tabcla 7.10 - Número de $\delta$ 's e Número de $\ell$ 's - Problemas Densos..................................111

Tabela 7.11 - Busca Completa_1 × Busca Parcial -- Problemas Densos............................112

Tabela 7.12 - Busca Completa_1 $1 \times$ Busca Completa_2 ……….....................................112

Tabela 7.13 - Busca Complcta_1 1 Sem Busca - Problemas Esparsos 4 Blocos...............114

Tabela 7.14 - Busca Complcta_1 $\times$ Regra de Armijo - Problcmas Esparsos 4 Blocos.....115

Tabela 7.15 - Busca Completa_ $1 \times$ Sem Busca - Problemas Esparsos 20 Blocos............115

Tabela 7.16 - Busca Completa_1 $\times$ Regra de Armijo - Problemas Esparsos 20 Blocos...116

Tabela 7.17 - Tempo Total $\times$ Tempo de Ordenação......................................................116

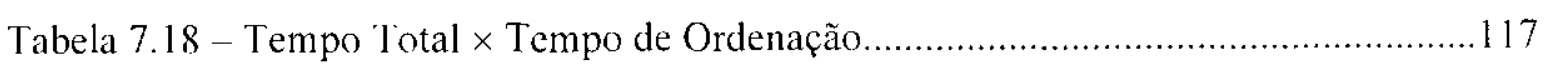

Tabela 7.19 - Número de $\delta$ s e Número de $\ell$ 's -4 blocos...............................................118

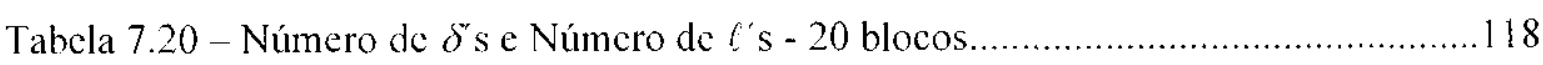

Tabela 7.21 - Busca Completa $1 \times$ Busca Completa_2-Problemas Esparsos 4 Blocos.....119

Tabela 7.22 ‥ Busca Completa_1 $\times$ Busca Complcta_2-Problemas Esparsos 20 Blocos...120

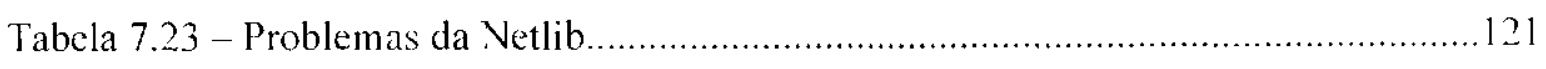

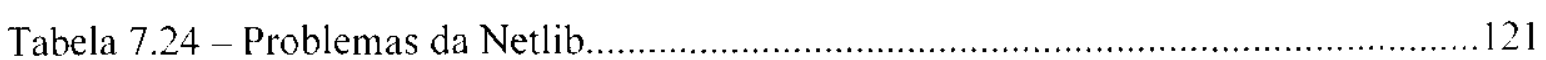

Tabcla 7.25 - Problemas degenerados resolvidos pelo DSBL usual...............................122

Tabela 7.26 - Problemas degenerados resolvidos pelo DSBU expansão...........................123

Tabela 7.27 - Problemas degenerados resolvidos pelo CPLEX .......................................123

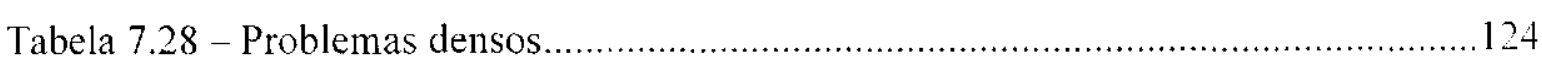

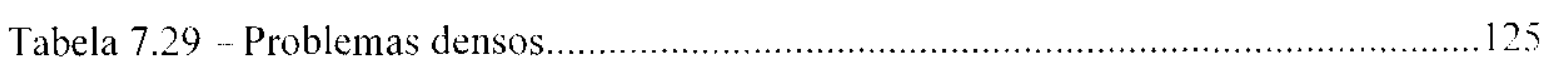

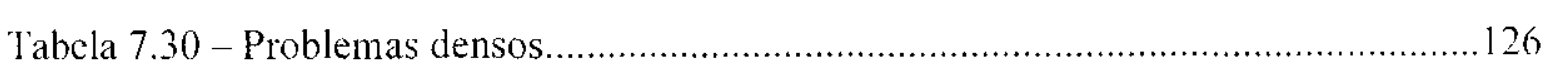

Tabela 7.31 - Dantzig $\times$ Dantzig Normalizada - Problemas Esparsos 4 Blocos................126

Tabela 7.32 - Dantzig $\times$ Dantzig Normalizada -.. Problemas Esparsos 20 Blocos.............127 
Tabela 7.33 - Problemas esparsos para caso de 20 Blocos..............................................128

Tabela 7.34 - Problemas esparsos com vários tamanhos de blocos...................................129

Tabela 7.35 - Problemas da Netlib resolvidos pelo CPLIXX............................................130

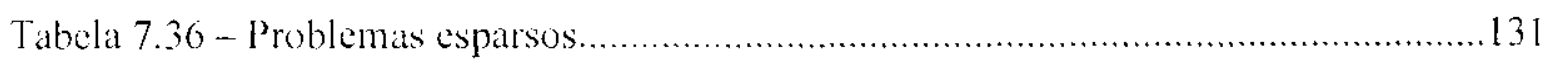

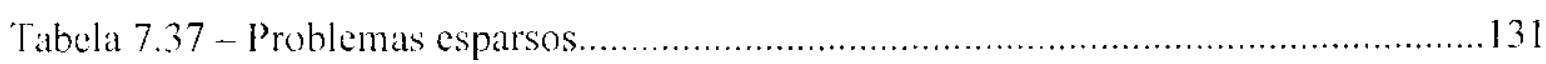

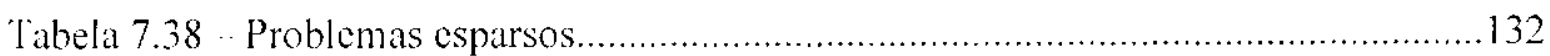

Capítulo 8. Conclusões e Trabalhos Futuros..................................................................133

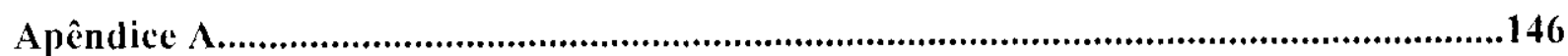

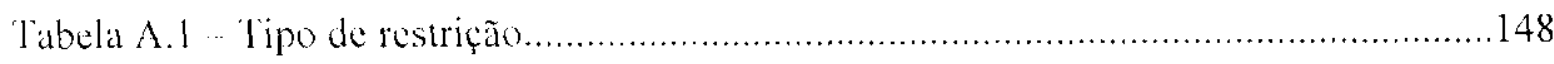

'Tabela A.2 Tipo de restrição e valores para os limitantes..............................................149

Tabela A.3 - Tipo de restrição e o significado............................................................. 149

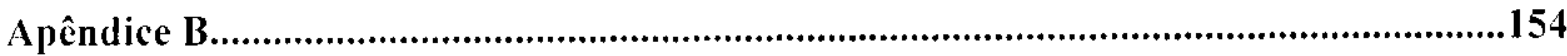




\section{Capítulo 1 - Introdução}

$\Lambda$ habilidade de modelar e resolver problemas de otimização linear tem um marco histórico cm 1947, sendo extraordinariamente ampliada com a evolução da ciência da computação. "Tão rápido tem sido o avanço em otimização que poucos lembram as contribuições de grandes pioneiros que começaram tudo isso" diz Dantzig (2002). Alguns deles são Von Neumann, Kantorovich, Leontief e Koopmans. Os dois primeiros foram matemáticos lamosos e os três últimos receberam o prêmio Nobel em economia em 1975, que segundo Todd (2001) caberia ao próprio Dantzig.

De acordo com Todd (2001), após o surgimento do método simplex em 1947, os anos cinqüenta foi a década de desenvolvimento de suporte teórico de otimização linear, de estender sua aplicabilidade na indústria e a certos problemas combinatoriais. Na década 60 emergiram problemas de grande porte, técnicas de explorar a estrutura especial de certos problemas (Dantzig et al. $1955 \mathrm{e}$ Dantzig e Wolfe, 1961) e de extensões à programação quadrática e complementaridade linear. Assim, as décadas de 50 e 60 foram vistas como um período de grande entusiasmo em otimização linear com o método simplex, mas segundo Todd, os anos 70 foram a década da dúvida, com a publicação de Klee e Minty (1972) de que o método simplex é exponencial, no estudo do pior caso.

A publicação de Khachiyan em 1979, propondo o método dos elipsóides com complexidade polinomial e do surgimento de métodos de pontos interiores (MPI), de Karmarkar (1984). intensificaram a pesquisa em otimização linear, reacendendo a questão da complexidade do método simplex, como por exemplo, limitantes para o número de iterações do método simplex com suposições probabilisticas nos dados dos problemas (Borgwardt, 1987). () aumento da capacidade de processamento e memória dos computadores e o avanço das técnicas de implementação, 
tornando os algoritmos mais eficientes, fizeram com que problemas práticos (dificeis de serem resolvidos) disponibilizados na Netlib (veja apêndice $\Lambda$ ) podem ser resolvidos por pacotes de otimizacao linear, como o CPLFX ou XPRESS. Tudo isso revigorou e afirmou a importância dos métodos de resolução da otimização linear. Os anos 80 foran uma década de grandes avanços computacionais para o método simplex. No capítulo 2, apresentamos mais detalhadamente os trabalhos também relevantes no contexto da história da otimização linear.

\subsection{O Problema de Otimização Linear Canalizado}

Uma classe de problemas de otimização linear de grande interesse prático é a classe dos problemas canalizados ou forma geral (Vanderbei, 1997) que podem ser formulados por:

$$
\begin{aligned}
& \text { Minimizar } f(x)=c^{\mathrm{J}} \boldsymbol{x} \\
& \text { Sujcito a: } \boldsymbol{d} \leq \boldsymbol{A x} \leq \boldsymbol{e} .
\end{aligned}
$$

O problema dual de (1.1) é um problema linear por partes e o método simplex pode ser especializado ao problema dual linear por partes, executando uma busca unidimensional na direção simplex (capitulo 3). A esta especialização chamamos de método dual simplex com busca unidimensional (DSBU) (Arenales, 1984 e Maros. 2003ㄱ). Em Sousa (2000), para exemplares de problemas na forma (1.1), com a matriz $\boldsymbol{A}$ densa gerados alcatoriamente, foi observado que a busca unidimensional linear por partes fe\% reduzir, sem um custo adicional, significantemente o número de iterações, inferior ao número de iterações do algoritmo dual simplex do pacote CPLEX 4.0.9, o qual era considerado uma das melhores implementações no método simplex. Isto nos motivou a investigar mais profundamente a abordagem dual-simplex com busca unidimensional. Investigamos também alternativas para o método ISSBU, como a direção simplex normalizada, que segundo (Forrest e (joldfarb, 1992) faz reduzir significantemente o número de iterações simplex cm alguns problemas de grande porte.

Neste trabalho aprofundamos a investigação sobre o método dual simplex com busca unidimensional, propondo e analisando variantes para a busca unidimensional linear por partes. exatas ou inexatas. que inclui a adaptação da regra clássica de Armijo e outras especílicas à função linear por partes, além de investigar problemas densos e esparsos. com algumas técnicas de resolução de sistemas lineares, incluindo o método do Gradiente Bi-Conjugado (GBC), que 
alimentou grande expectativa no aumento da eficiência computacional para problemas de grande porte.

Bixby et al., (2000) realizaram testes computacionais para os métodos primal simplex, dual simplex e um método de pontos interiores primal-dual e os autores concluíram que, para uma séric de problemas grandes, o método dual simplex com a regra de Danzig Normalizada foi o mais rápido. Eles colocaram algumas questões em aberto sobre a eficiência dos métodos de otimização linear: "O que se pode dizer e'm geral sohre a methor maneira de resolver problemas grandes? Qual algoritmo é o methor? Se estas questões fossem colocadas $\mathrm{cm} 1998$, nossa reposta seria que o métode de pontos interiores com harreira era claramente o melhor para resolver problemas grandes. Se ela fosse respondida agora, nossa resposta seria que não está claro qual é o melhor ulgoritmo". Segundo Bixby et al. (2000) (conforme citado por Todd, 2001). cada um dos métodos primal simplex, dual simplex e de pontos interiores é superior em um número significante de exemplares importantes de otimização linear.

Portanto, o campo da pesquisa em métodos (primal e/ou dual) do tipo simplex e de pontos interiores é ativo e de grande interesse e pode contribuir muito para otimização linear, com todas as implicações na otimização inteira e não-linear.

De acordo com Maros (2003), o método dual simplex tem atraído considerável interesse também. particularmente devido à importante aplicação nos métodos de otimização linear inteiro misto, os quais resolvem uma seqüência de problemas de otimização linear, com característica de que uma solução básica dual factível de boa qualidade é sempre disponível para o problema seguinte da sequiência. Segundo Bixby (2001), testes computacionais mostram que o desempenho do método dual simplex pode ser superior ao método primal simplex.

O problema central desta tese, que é resolver problemas de otimização linear canalizado, tem sido pouco abordado na literatura. Esse modelo tem particular interesse, não só por representar facilmente qualquer problema de olimização linear, como também por ser uma forma que surge naturalmente com as técnicas de pré-processamento, quando limitantes naturais sobre as variáveis são utilizados para 'apertar' os limitantes das restrições.

O Método Dual Simplex foi também escollhido como ferramenta de resolução, por ser mais 'natural' para problemas canalizados, uma vez que quanto mais restrito o problema primal, mais relaxado é o problema dual.

Algumas técnicas foram adaptadas para o método dual simplex com busca unidimensional (I)SB(1) com o objetivo de tratar a esparsidade que se faz presente em muitos problemas práticos e de grande porte. 
Foram objetos de estudo desta tese também, a decomposição $L U$ com uso de heurísticas e os métodos de atualização da base de Forrest e Tomlin (1977) c Reid (1982).

No cenário de problemas canalizados e csparsos. apresentamos o método dual simplex com busca unidimensional utilizando o método iterativo do gradiente bi-conjugado para resolver os sistemas lineares a cada itcração, para o qual não temos conhecimento de nenhum trabalho na literatura de otimização linear com o uso do método simplex. Este método iterativo é tão competitivo, que para a grande maioria dos problemas que resolvemos, comportou-se equivalente ao pacote CPLEX 7.5, que é considerado uma das melhores implementações dos métodos lipo simplex, em particular, o método dual simplex.

\subsection{Organização do Texto}

O texto está dividido $\mathrm{em} 8$ capítulos e 2 apêndices de acordo com os seguintes conteúdos:

Neste capítulo, demos uma pequena introdução da história da ()timização linear (OL) que será descrita em mais detalhes no capitulo 2 e destacamos o problema canalizado de OL que trabalhamos $\mathrm{e}$ as ferramentas que foram utilizadas nos métodos tipo dual simplex que desenvolvemos.

No capítulo 2, inicialmente uma breve história de otimizaçăo linear é descrita e apresentamos o modelo clássico, destacando-se os trabalhos mais relevantes na literatura. Além disso, relatamos sobre o desempenho do método simplex de Dantzig e apresentamos uma seção para os métodos de pontos interiores que são uma outra classe de métodos para resolver os problemas de otimizacao linear eficientemente.

No capítulo 3 é apresentado o desenvolvimento do método dual simplex com busca unidimensional (DSBU) especializado em resolver os problemas de otimização linear canalizados. c onde propomos três tipos de busca unidimensional.

No capitulo 4, relatamos primeiramente os sistemas lineares esparsos e as heurísticas utilizadas para decompor a base utilizando a decomposição LU (método direto). pois a cada iteração do método simplex três sistemas lineares precisam ser resolvidos, cuja resolução representa a grande parte do gasto computacional do método simplex, o que torna importante a investigação na resolução desses sistemas, principalmente $\mathrm{cm}$ problcmas de grande porte e esparso. Em seguida. são apresentados os métodos mais eficientes para atualização da base no método simplex, aproveitando a decomposição já realizada. Por último, apresentamos o método iterativo do Gradiente BiConjugado (GBC), que incorporamos na implementação do método dual simplex com busca unidimensional. 
No capítulo 5 é relatado o problema de degeneração com risco de ciclagem no método simplex, com apresentação de algumas técnicas para evitar tal fenômeno. Em seguida, relatamos brevemente algumas variantes simplex e apresentamos a regra de Dantzig Normalizada para o método dual simplex com busca unidimensional. Veremos nos experimentos computacionais (capítulo 7) que tais técnicas, além de inibirem a ciclagem, introduz um efeito positivo para diminuir a estagnação do método (repetição de bases oriundas ao mesmo vértice)

No capítulo 6, são apresentadas as estruturas de dados que utilizamos nas implementações das buscas unidimensionais descritas no capitulo 3 e dos métodos descritos no capítulo 4.

No capítulo 7 apresentamos os experimentos computacionais obtidos com as heurísticas para decomposição da base e métodos de atualização. utilizando-se a decomposição LU, com as buscas unidimensionais propostas e, para o DSBU utilizando o método do gradiente bi-conjugado.

No capitulo 8 apresentamos as conclusões do trabalho $\mathrm{c}$ as pesquisas futuras nesta área de conhecimento.

No apêndice A procuramos detalhar os formatos de arquivo lp e mps para os problemas de Otimização I inear, cm seguida uma rápida descrição sobre os pacotes comerciais de otimização linear: CPIEX e XPRESS.

Por ultimo. no apêndice B, apresentamos resumidamente o desenvolvimento do clássico método primal simplex para problemas de otimização linear na forma padrão e as relações primaldual. Limbora estes tópicos sejam bem difundiưos, entendemos que estas notações e nomenclaturas podem auxiliar na leitura deste texto. 


\section{Capítulo 2 - Otimização Linear: História, Métodos Tipo Simplex e de Pontos Interiores}

\subsection{Um Breve Histórico de Otimização Linear}

A otimização linear consiste no cstudo (desenvolvimento de teoria e métodos de solução) de problemas de minimização ou maximização de uma função linear, definida sobre um domínio descrito por equações e/ou inequações lineares, que pode ser representada por:

$$
\begin{aligned}
& \text { Minimizar } f(x)=c^{\top} x \\
& \text { Sujeito a: } A \boldsymbol{x}=\boldsymbol{b} \\
& x \geq 0,
\end{aligned}
$$

em que $\boldsymbol{A}$ é uma matriz $m \times n, c$ um vetor $n \times 1$ e $\boldsymbol{b}$ um vetor $m \times 1$. A notação $\boldsymbol{x} \geq \boldsymbol{0}$ quer dizcr: $x_{j} \geq 0, j=1, \ldots, n$. A função linear $f: \mathrm{R}^{n} \rightarrow \mathrm{R}$ é chamada função objetivo. Uma solução que satisfaça as restrições $\boldsymbol{A x}=\boldsymbol{b}, \boldsymbol{x} \geq \boldsymbol{0}$ é dita solução factível.

Resolver um problema de otimização linear, consiste em encontrar uma solução factível $\boldsymbol{x}^{*}$ tal que, $f\left(x^{*}\right) \leq f(x)$ para toda solução factível $x$. Dizemos que $x^{*}$ é uma solução ótima.

A história da otimização linear é marcada a convivência com situações que estimulam a procura de métodos para otimizar o uso de recursos escassos, visando compartilhá-los entre 
atividades competitivas. Embora de origem menos recente, convencionou-se como o início da pesquisa operacional, o período da segunda grande guerra, quando problemas estratégicos e táticos de origem militar foram solucionados. Aplicações industriais de sucesso se sucederam.

Grandes organizações, como as companhias de petróleo, foran as primeiras a adotar o novo conjunto de metodologias para a resolução de seus problemas de decisão, crescendo o uso da programação linear no planejamento da produção em larga escala. Mais tarde, pequenas companhias puderam usufruir do conhecimento acumulado. $\Lambda$ otimização linear, como instrumento de planejamento e gestão ótima de sistemas, é um dos tópicos mais difundido e utilizado da pesquisa operacional.

O problema de otimização linear foi formalizado por George B. Dantzig em 1947 que. cm seguida, desenvolveu o Método Primal Simplex para resolvê-lo. (Veja o apêndice B para o desenvolvimento do método). Desde então, um número grande de pesquisadores tem contribuído para o campo da otimização linear de diferentes maneiras, incluindo desenvolvimentos teóricos, aspectos computacionais e novas aplicações. Muitas variantes do método simplex foram e, ainda hoje, são desenvolvidas.

A abordagem pela otimização linear desfruta de ampla aceitação devido a habilidade de modelar importantes e complexos problemas na tomada de decisões e da capacidade de seus métodos para produzir soluções em tempo razoável. Hoje em dia, problemas com milhares de restrições e variáveis são resolvidos em microcomputadores.

Klee e Minty em 1972 apresentaram um exemplar, para o qual o método simplex necessita de $2^{n}-1$ iterações, percorrendo todos os vértices da região factível do problema, a qual é definida como sendo uma distorção do hipercubo $n$-dimensional que tem $2^{n}$ vértices.

Matematicamente, o problema em $\mathrm{R}^{n}$ pode ser descrito da seguinte forma:

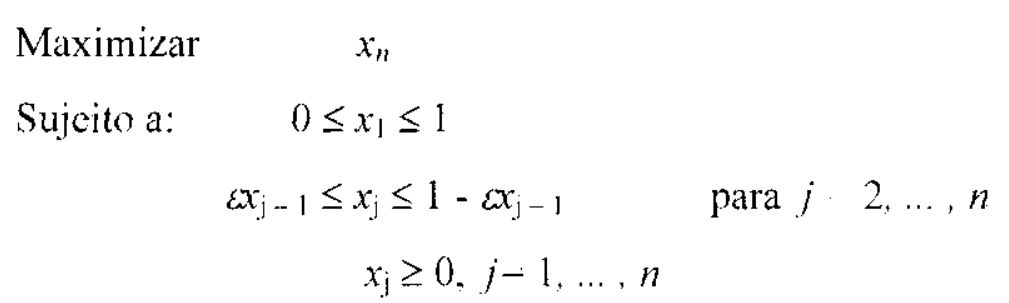

em que $\varepsilon e ́$ um número racional no intervalo $(0,1 / 2)$.

Após uma transformação sutil deste problema (Bazaraa et. al., 1990), usando a regra de Dantzig e tendo como uma solução básica factível inicial a origem, o algoritmo simplex caminha em $2^{n}-1$ arestas desse politopo e visita todos os $2^{n}$ vértices da região factível. 
Assim, temos que algoritmo simplex não pertence à classe de algoritmos com tempo polinomial. $\Lambda$ Figura 2.1 representa o caso $n=3$, em que a região factível é a região interna do cubo deformado, dado pela seguinte formulação (em formato .lp, veja apêndice $A$ ):
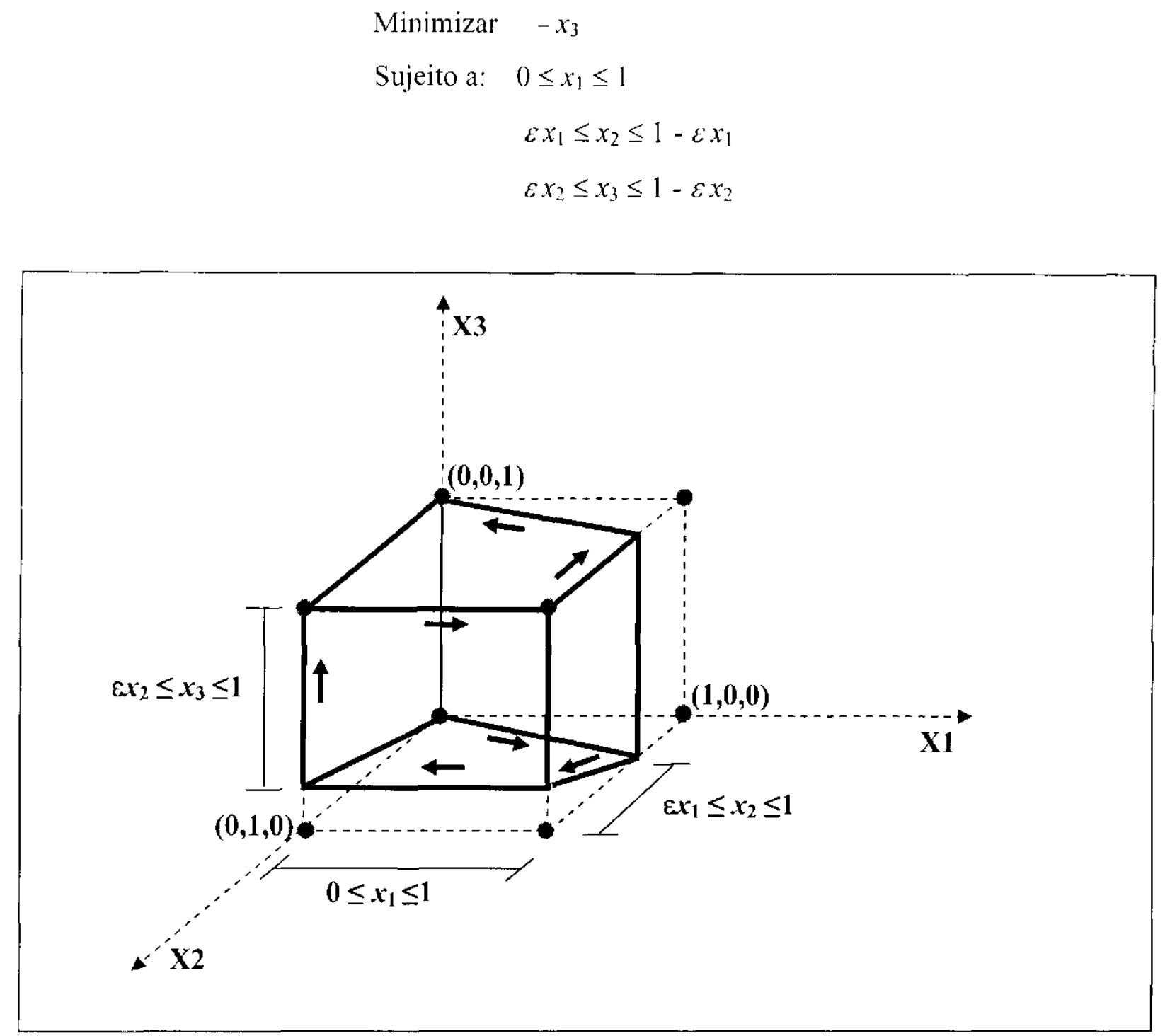

Figura 2.1-Exemplar de Klee e Minty com três variáveis

Também foi possivel construir excmplares, para os quais as principais variantes do método simplex também exccutou um número exponencial de iterações (Terlaky e 7hang, 1993).

No final da década de setenta, Khachiyan (1979) apresentou o Algoritmo dos Elipsóides para a otimização linear, com tempo polinomial. Este trabalho resolveu uma questão teórica da época, colocando problemas de otimização linear na classe de problemas polinomiais. Porém, o desempenho do novo algoritmo foi ineficiente, pois o número de iterações tende a ser muito grande 
e a quantidade de cálculos associados em cada iteração é muito maior comparado com o método simplex. Além disso, o algoritmo teve problema de instabilidade numérica.

Mais tarde, Karmarkar (1984) publicou um Método de Pontos Interiores capaz de encontrar uma solução ótima em tempo polinomial. Além disso, o autor afirmou que seu método cra muito mais rápido do que o método simplex (em alguns casos 50 vezes), o que estimulou uma avalanchc de pesquisa na área de olimização linear, incluindo-se questões desafiadoras sobre a efíciência computacional de métodos do tipo simplex. Segundo Dantzig (Dantzig e Thapa, 1997) nos primórdios da otimização linear, Von Neumann propôs um método que hoje seria chamando de pontos interiores, porém as implementações feitas na ćpoca deram grande vantagem computacional ao método simplex, inibindo as pesquisas nessa direção.

\subsection{A Eficiência e a Complexidade Computacional do Método Simplex}

A questão da eficiência do método simplex sempre loi tema de pesquisa desde a sua publicação. No início, a convergência finita do método, garantida com regras que evitassem ciclagem, era suficiente para os pesquisadores, apesar do número de iterações ser muito grande.

No algoritmo simplex, o principal passo computacional é a resolução dos sistemas básicos a ser realizado a cada iteração, que pode ser feito $\mathrm{em} O\left(\mathrm{~m}^{3}\right)$ operações elementares com método de eliminação de Gauss. $\Lambda$ ssim, o esforço necessário para resolver (2.1), pelo algoritmo simplex pode ser medido pelo número de iterações.

Algumas questões surgiram: Qual é o número médio de iterações para resolver um problema de otimização linear? É possível expressar este número médio como uma função de $m$ e $n$ ? Esta função é conhecida como complexidade computacional média do algoritmo simplex.

Da experiência computacional com um número grande de problemas resolvidos em vários anos (Murty, 1983), uma resposta empírica para estas questões surgiu, indicando que o número médio de iterações é uma função linear de $m$, que parece ser menor do que $3 m$. Segundo Shamir (1987), na maioria dos problemas reais, o número de iterações seria em média $\alpha m$, em que $\alpha$ é um valor entre 2 e 3 . De diversas experiências computacionais, surge um folclore de que o número médio de iterações do método simplex é uma função polinomial de $m$, de grau baixo.

Reforçando este folclore, um estudo computacional para o problema bidimensional de corte de peças em estoque (que envolve milhares de variáveis, Gilmore e Gomory, 1965) mostra que o número de iterações do método simplex é da ordem de $m^{2}$ (Pinto, 1999 e Sousa, 2000). 
As Tabelas 2.1 e 2.2 mostram o número de iterações para diferentes valores do número de tipos de peças $M$ ( $M$ fornece o número de restrições do modelo de Gilmore e Gomory e cada coluna corresponde a uma maneira particular de se cortar uma placa em estoque, chamada padrão de corte').

Se os tamanhos dos itens a serem cortados são pequenos, à placa em estoque, então o númcro de padrões de corte (i.e., número de variáveis do modelo) é grande. dizemos que o problema é grande: caso contrário, dizemos que é pequeno. Se escrevermos a relação linear IT = $\alpha M$ (IT é o número de iterações), vemos que $\alpha$ é uma função linear de $M$, isto ć, $\alpha=\beta M$ sendo que para problemas pequenos $\beta \cong 0,12$ e para problemas grandes $\beta \cong 0,21$. Em resumo $\mathrm{IT}=\beta M^{2}$ (veja Figura 2.1).

Tabela 2.1 - Exemplos Pequenos

\begin{tabular}{|c|c|c|}
\hline$M$ & $\alpha$ & $1 T$ \\
\hline 5 & 0.812 & 4,06 \\
\hline 10 & 1.122 & 11,22 \\
\hline 20 & 2,164 & 43,28 \\
\hline 40 & 5.094 & 203,76 \\
\hline
\end{tabular}

Tabela 2.2 - Exemplos Grandes

\begin{tabular}{|c|c|c|}
\hline$M$ & $\alpha$ & $M$ \\
\hline 5 & 1,28 & 6,4 \\
\hline 10 & 2,112 & 21,12 \\
\hline 20 & 3,976 & 79,52 \\
\hline 40 & 8,006 & 320,24 \\
\hline
\end{tabular}
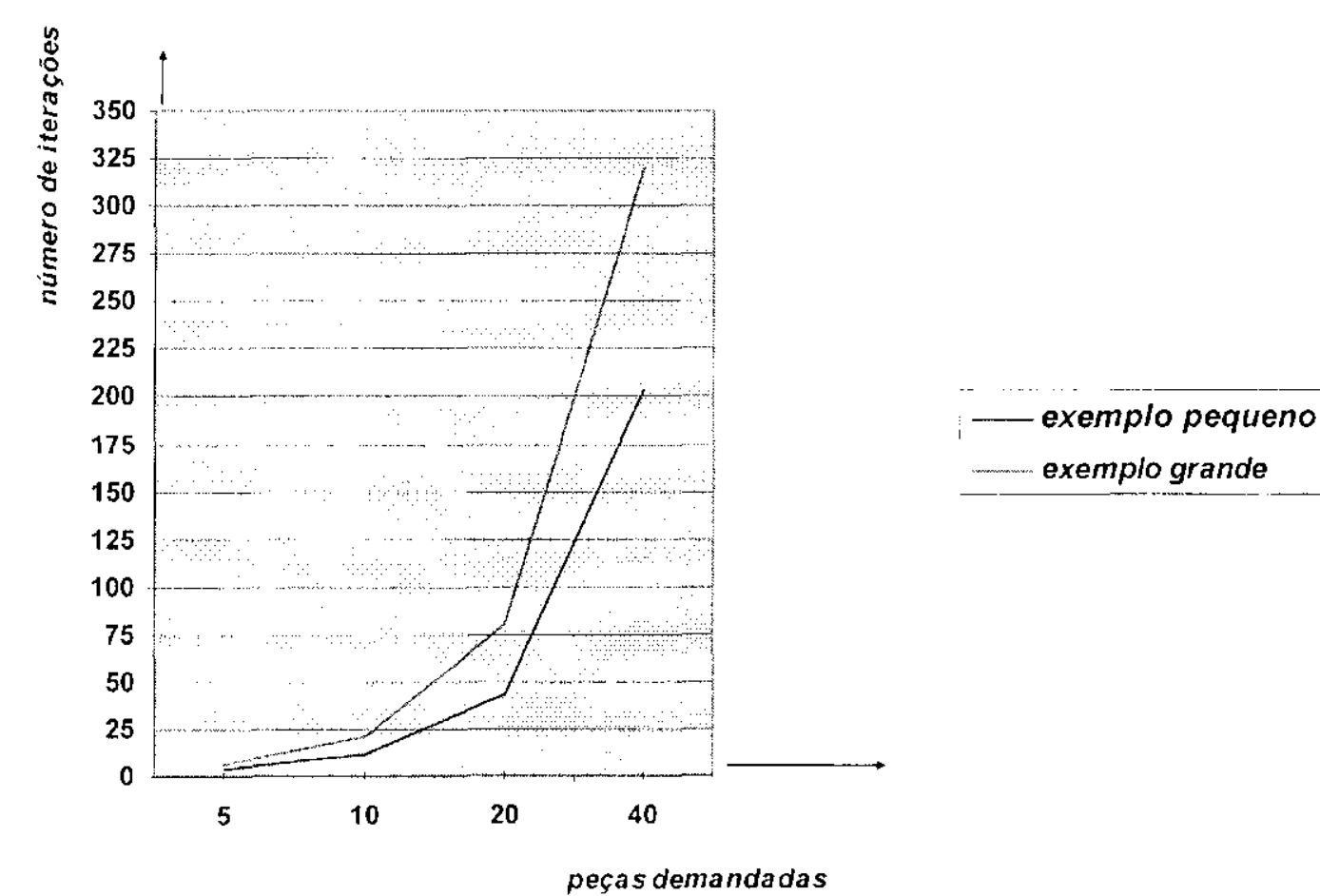

Figura 2.2 - Desempenho médio do método simplex aplicado ao problema de corte 
Como já observamos, a complexidade computacional com o estudo do pior caso. classifica o método simplex como um algoritmo não-polinomial (já que existe um exemplar que cxigc um número exponencial de iterações), embora, como também observamos, seu desempenho médio é polinomial, principalmente para problemas práticos típicos.

As principais variantes do método simplex também são classificadas como não-polinomiais. no estudo do pior caso. Surge então, uma questão instigante (Segundo Tcrlaky e Zhang, 1993, a questão aberta mais instigante da teoria de otimização linear): existe um algoritmo do tipo simplex (i.e. que percorre soluções básicas) que seja polinomial nos dados do problema?

É importante observar que a evolução das implementações computacionais do algoritmo do tipo simplex teve um papel fundamental no progresso da Otimização I.inear. Um exemplo disto é o pacote CPLEX, que vem sendo melhorado desde sua primeira versão lançada em 1988. Bixby (1992) apresentou resultados para O CPLEX $1.0 \mathrm{em} 90$ problemas da Netlib. Para 72 problemas, o número total de iteraçōes foi no máximo 3 vezes o tamanho das restrições: para 16 problemas a razão foi entre 3 e 7 c para os três problemas restantes esta razão foi 10.7 .39 .5 e $469.1 \mathrm{com}$ dimensões $1000 \times 8806,24 \times 1026$ e $25 \times 10500$ respectivamente, sendo que os dois últimos todas as variáveis têm limitantes superior e inferior. E, em um conjunto de 8 problemas grandes Bixby (1994) observou que as razões para o CPLEX 2.2 foram de 0.46 a 1.99 em três problemas; de 4.52 a 9.02 em 4 problemas e 17.58 no último. Fsses problemas (especialmente o maior deles) foram escolhidos por apresentar dificuldades para os algoritmos de otimização linear. Assim, segundo Todd (2001) o desempenho favorável deles é então surpreendente.

Bixby (2001) também relatou que a implementação do método dual simplex, na primcira versão do pacote CPLEX 1.0 rodado numa estação de trabalho CitraSparc de $300 \mathrm{MHz}$, resolvia um problema de 16223 restrições e 28568 variáveis, com 88340 elementos não nulos em 1217.4 segundos e, o mesmo método na última versão CPLEX 7.5 na mesma máquina, resolvia o mesmo problema em 22,6 segundos. Ou seja, um mesmo método pode se tornar 50 veres mais rápido dependendo de sua implementação. Vale observar que esta relação de desempenho também foi obtida por Karmarkar (1984). que alirmou que o método de pontos interiores era 50 vezes mais rápido do que o método simplex. Obviamente, estruturas de dados adequadas são fundamentais para o desempenho de uma implementação computacional. A despeito disto. um mesmo método pode permitir um número grande de variantes, com desempenhos bastante diversos, como por exemplo, a regra de Dantizg Normalizada apresentada no capítulo 5 (conhecida na literatura de língua inglesa por 'steepest edge rule'. Veja Forrest e Goldfarb, 1992). 


\subsection{Métodos de Pontos Interiores}

\subsubsection{Introdução}

Esta seção sobre método de pontos interiores, está bascada no trabalho de Freund e Mizuno (2000), que apresentam um panorama de tais métodos no campo da otimização linear que até 1984 foi dominado pela pesquisa em métodos tipo simplex (Terlaky e Zhang, 1993).

Karmarkar (1984) apresentou um método de Pontos Intcriores polinomial, e a partir dai a pesquisa em otimização linear se concentrou nesses métodos, sendo que mais de 3000 artigos foram escritos no assunto, $\mathrm{c}$ várias implementações eficientes comerciais e científicas foram feitas.

Segundo Roos e Terlaky (1996). depois de uma intensa pesquisa em métodos de pontos interiores tanto na teoria como na prática, esses métodos são significantemente mais eficientes do que o método simplex, em alguns problemas de otimização linear, perfazendo 10 a 40 iterações.

Métodos de pontos interiores mudaram o panorama da teoria, da prática e da computação em progranação matemática. A otimização linear não é mais sinônimo do clássico método simplex e muitos pesquisadores tendem a ver agora este campo de pesquisa mais como um caso especial de otimização não linear devido ao desenvolvimento desses métodos.

Segundo Freund e Mizuno, a "pedagogia" de métodos de pontos interiores atrasou parcialmente sua popularidade até há pouco tempo, porque estes métodos (i) usam ferramentas matemáticas mais avançadas do que métodos do tipo simplex. (ii) a análise matemática é tipicamente muito mais complicada, e (iii) us métodos são menos receptivo à intuição gcométrica. Até pouco tempo, os educadores lutaram com a questão de como e onde introduzir métodos de pontos interiores no curriculo de programação linear e não lincar e como convincentemente cxpor tais métodos para estudantes (e pesquisadores da mesma categoria). Como a pesquisa em métodos de pontos de interiores para programação linear se acalmou (e a pesquisa em pontos interiores para programação não linear aqueceu), vários projetos de livro novos em programação linear e/ou métodos de pontos interiores apareceram recentemente no cenário, prometendo superar estas dificuldades. 


\subsubsection{Os Mćtodos de Pontos Interiores}

Para introduzir tais métodos para otimização linear. considere o para primal-dual:

$$
\begin{aligned}
& \text { Minimizar } c^{\top} x \\
& \text { Sujeito a: } \boldsymbol{A x}=\boldsymbol{b} \\
& x \geq 0
\end{aligned}
$$

em que $\boldsymbol{A}$ é uma matriz $m \times n$,

$$
\begin{aligned}
& \text { Maximizar } \boldsymbol{b}^{\mathrm{T}} \boldsymbol{w} \\
& \text { Sujeito a: } \boldsymbol{A}^{\mathrm{T}} \boldsymbol{w}+\boldsymbol{s}=\boldsymbol{c} \\
& \qquad \boldsymbol{s} \geq \boldsymbol{0}
\end{aligned}
$$

Dada uma solução factível $\boldsymbol{x}$ de (P) e uma soluçâo factível $(\boldsymbol{w}, \boldsymbol{s})$ de (D), o gap de dualidade é facilmente expresso por:

$$
\begin{aligned}
& c^{\mathrm{T}} x-b^{\mathrm{T}} w=c^{\mathrm{T}} x-x^{\mathrm{T}} A^{\mathrm{T}} w \\
& c^{\mathrm{T}} \boldsymbol{x}-b^{\mathrm{T}} w=x^{\mathrm{T}}\left(c-\boldsymbol{A}^{\mathrm{T}} w\right) \\
& c^{\mathrm{T}} \boldsymbol{x}-\boldsymbol{b}^{\mathrm{T}} w=x^{\mathrm{T}} s \geq \mathbf{0} .
\end{aligned}
$$

O gap de dualidade é zero se, e somente se, $\boldsymbol{x}$ é ótimo $\mathrm{cm}$ (P) e $\boldsymbol{w}$ é ótimo em (D). Assim, o gap de dualidade serve como uma boa medida para verificar o quanto as soluções factíveis $\mathbf{x}$ e $\mathbf{w}$ estão próximo das soluções ótimas para (P) e (D).

As condições do teorema 7 (tcorema das folgas complementares, apêndice B) para o par (P) - (D) podem ser equivalentemente escritas por uma das propriedades:

1. $\left(s^{*}\right)^{\mathrm{T}} \boldsymbol{x}^{*}=0$

2. $x^{*}, s^{*}{ }_{j}=0 \quad j=1, \ldots, n$;

3. Se $s_{j}^{*}>0$ então $x_{j}^{*}=0$.

Utilizaremos a seguinte notação mais conveniente para o desenvolvimento de métodos de pontos interiores. Uma solução factível $x$ de $(\mathrm{P})$ é interior se $\boldsymbol{x}>\boldsymbol{0}$ e uma solução factível $(\boldsymbol{w}, \boldsymbol{s})$ de 
(D) é interior se $s>0$. Seja $\hat{e}=(1, \ldots, 1)^{\mathrm{T}}$. A matri $X$ é definida como sendo a matriz diagonal $n$ $\times n$, em que cada elemento da diagonal é justamente as componentes do vetor $\boldsymbol{x}$, isto é:

$$
\boldsymbol{X}=\left(\begin{array}{cccc}
x_{1} & 0 & \cdots & 0 \\
0 & x_{2} & \cdots & 0 \\
\vdots & \vdots & \ddots & \vdots \\
0 & 0 & \cdots & x_{n}
\end{array}\right)
$$

Note que $X \hat{\boldsymbol{e}}=\boldsymbol{x}$, e (supondo $\boldsymbol{x}>\boldsymbol{0}) X^{1} \hat{\boldsymbol{e}}=\left(1 / x_{1}, \cdots, 1 / x_{n}\right)^{\mathrm{T}}$, e que $X$ e $X^{-1}$ são matrizes simétricas definidas positivas.

A maioria dos métodos de pontos interiores estão numa das três principais categorias: Métodos Afim-Escala, Métodos de Redução Potencial, e Métodos da Trajetória Central. Resumiremos brevemente estas três categorias.

a) Métodos Afim-Escala. A estratégia básica do método Alim-Escala é: dada uma solução interior $\bar{x}$ de $(P)$. construa um elipsóide simples local $E_{\bar{x}}$ na região factível de $(P)$ centrado $\mathrm{em} \overline{\boldsymbol{x}}$. Então. otimize a função objetivo $c^{\mathrm{T}} x$ sobre $E_{\bar{x}}$, e use a direção resultante com um passo apropriado para definir um nova solução. As particularidades desta estratégia são:

Dada solução interior $\bar{x}$ de (P), o Elipsóide em $\bar{x}$ é definido como:

$$
E_{\bar{x}}=\left\{\boldsymbol{x} \in R^{n} \mid \boldsymbol{A x}=\boldsymbol{b},(\boldsymbol{x}-\overline{\boldsymbol{x}})^{\mathrm{T}} \bar{X}^{-2}(\boldsymbol{x}-\overline{\boldsymbol{x}}) \leq 1\right\}
$$

em que $\bar{X}^{2}$ é o quadrado de $\bar{X}^{-1}$ para o ponto interior $\bar{x}$ (esta vizinhança de $\bar{x}, E_{\bar{x}}$ está contida na região factível de $(P)$ pois $\bar{x}$ é interior.) $A$ direção $\Lambda$ fim $\mathrm{em} \bar{x}$ é então a solıção do seguinte problema:

$$
\begin{array}{r}
\text { Minimizar } c^{\mathrm{T}} d \\
\text { Sujeito a: } \quad \boldsymbol{A} d=0 \\
\boldsymbol{d}^{\mathrm{T}} \bar{X}^{-2} \boldsymbol{d} \leq 1
\end{array}
$$

Note que $(\mathbf{P} \bar{x})$ é um problema convexo com todas as restrições lineares exceto para uma restrição quadrática convexa. Pode ser resolvido analiticamente, a partir das condições necessárias e 
suficientes de $1^{\text {a }}$ ordem de Karush-Kuhn-Tucker. A solução $\overrightarrow{\boldsymbol{x}}$ é perturbada na direção $\boldsymbol{d}$, com um passo que mantém o novo ponto interior e repete-se o procedimento.

Segundo Freund e Mizuno (2000), este método é atracnte devido a sua simplicidade e seu bom desempenho na prática. (Porém. seu desempenho é bastante sensivel para o ponto inicial do algoritmo.) Não existe qualquer resultado sobre complexidade computacional. mas há fortes indícios que o algoritmo seja exponencial no pior caso.

Algumas variantes/extensões no algoritmo básico Afim-Escala é o algoritmo Afim-Escala Dual (desenvolvido para desigualdades, veja Adler et al. 1986 e Balbo 1991), como também várias versões que trabalham simultaneamente no primal e no dual, usando uma variedade de construções do elipsóide no espaço primal e dual.

b) Métodos de Redução Potencial. Métodos de redução potencial são tipicamente projetado para encontrar soluçôcs melhores para o seguinte problema de otimização não linear:

\section{Problema de Redução Potencial (PRP)}

$$
\begin{gathered}
\text { Minimizar } \quad f(\boldsymbol{x}, \boldsymbol{w}, \boldsymbol{s})=q \ln \left(\boldsymbol{c}^{\mathrm{T}} \boldsymbol{x}-\boldsymbol{b}^{\mathrm{T}} \boldsymbol{w}\right)-\sum_{j-1}^{n} \ln \left(x_{j}\right) \\
\text { Sujeito a : } \begin{array}{c}
\boldsymbol{A} \boldsymbol{x}=\boldsymbol{b} \\
\boldsymbol{x}>\boldsymbol{0} \\
\boldsymbol{A}^{\mathrm{T}} \boldsymbol{w}+\boldsymbol{s}=\boldsymbol{c} \\
\boldsymbol{s} \geq \boldsymbol{0}
\end{array}
\end{gathered}
$$

em que a função objetivo $f(x, w, s)$ é chamada a função potencial, e $q$ um parâmetro. Foi este tipo de problema que Karmarkar trabalhou em scm artigo. Note que a "primeira parte" da função potencial é $q$ vezes o logaritmo do gap de dualidade, e deseja-se conduzir esta parte para $-\infty$ A Segunda parte da função potencial é a função barreira logaritma introduzida para "repelir" soluções na fronteira da região factivel.

O algoritmo original de Karmarkar (1984) usou uma forma muito específica de (PRP) e também o procedimento de transformações projetivas no algoritmo e na prova de garantia do desempenho do algoritmo (Gonzaga 1992). Segundo Freund e Mizuno (2000). apesar da mística original, transformações projetivas não são necessárias para algoritmos de redução potencial. sça na teoria como na prática. porém, nas opiniões de alguns autores, a estrutura de trabalho de 
transformações projetivas é, contudo, de suprema importância, pelo menos conceitualmente, na compreensão de métodos de pontos interiores em geral.

Em geral, todo método de redução potencial tem como objetivo conduzir a função potencial para - $\infty$ usando uma variedade de algoritmos primal, dual ou primal-dual. Quase todos métodos de redução de potencial desfrutam do desempenho garantido de complexidade polinomial. Métodos de redução de potencial não receberam muita atenção em termos de testes computacionais, devido talvez às dificuldades anteriores (que foram superadas) de aplicar métodos de redução potencial $\mathrm{em}$ um ambiente combinando Fase I e Fase II. Para um estudo aprofundado sobre esses métodos, referencia-se o trabalho de Todd (1997).

c) Métodos de Trajetória Central. Métodos de pontos interiores baseados na trajetória central são, sem dúvida, os mais úteis na teoria, e o mais usado na prática. A trajetória central do problema linear $(\mathrm{P})$ é obtida como a solução para uma versão compensada de $(\mathrm{P})$, em que um termo barreira logarítmica parametrizada é somado à função objetivo. Considere o problema de barreira logarítmico BP $(\mu)$, parametrizado em $\mu>0$ :

$$
\begin{aligned}
& \text { Minimizar } \quad c^{\mathrm{T}} x-\mu \sum_{j=1}^{n} \ln \left(x_{j}\right) \\
& \text { Sujeito a : } \quad \boldsymbol{A} \boldsymbol{x}=\boldsymbol{b} \\
& x>0
\end{aligned}
$$

As condições necessárias e suficientes de $1^{a}$ ordem de Karush-Kuhn-Tucker para BP( $\mu$ ) são:

$$
\left\{\begin{array}{l}
A x=b, x>0 \\
c-\mu X^{-1} \hat{e}=A^{\mathrm{T}} w
\end{array}\right.
$$

Se definirmos $s=\mu X^{-1} \hat{e}$, então podemos reescrever essas condições de otimalidade como;

$$
\left\{\begin{array}{l}
A x=b, x>0 \\
A w+s=c, \quad s>0 \\
X S \hat{e}-\mu \hat{e}=0
\end{array}\right.
$$


Seja $(x(\mu), w(\mu), s(\mu l))$ a solução para o sistema (2.3) para um dado parâmetro $\mu$ positivo. Então o conjunto $I^{\prime}=\{(x(\mu), w(\mu), s(\mu))$ tal que $\mu>0\}$ é definido como trajetória central do problema linear (P). Das duas primeiras equaçõcs do sistema (2.3), vemos que uma solução $(\boldsymbol{x}, \boldsymbol{w}, \boldsymbol{s})$ ao longo da trajetória central é interior para o problema primal e para o problema dual, e que o gap de dualidade na trajetória central é $\boldsymbol{x}^{\top} \boldsymbol{s}=\hat{\boldsymbol{e}}^{\top} \boldsymbol{X} \boldsymbol{S} \hat{\boldsymbol{e}}=\mu \hat{\boldsymbol{e}}^{\top} \hat{\boldsymbol{e}}=\mu \mathrm{n}$, que segue da terceira equação do sistema (2.3). Substituindo esta equação na terceira do sistema (2.3), obtemos a seguinte caracterização da trajetória central, livre de parâmetro que é equivalente ao anterior:

$$
\left\{\begin{array}{l}
A x=b, x>0 \\
A^{\mathrm{T}} w+s=c, s>0 \\
X S \hat{e}-\left(x^{\mathrm{T}} s / n\right) \hat{e}=0
\end{array}\right.
$$

A terceira equação no sistema (2.3) ou (2.4) não é linear e, em geral, não é possível resolvcr (2.3) ou (2.4) de forma fechada, exceto no caso trivial.

A estratégia na maioria dos métodos de trajetória central consiste em obter soluções aproximadas da trajetória central e diminuir. na seqüência, o gap de dualidade (ou equivalentemente, do parâmetro de barreira $\mu$ ) que tende a se anular no limite. Há várias maneiras para realizar esta estratégia. Por exemplo, para um dado valor do gap de dualidade ou do parâmetro barreira $\mu$, pode-se escolher aproximadamente otimizar $\mathrm{BP}(\mu)$ ou, equivalentemente, resolver aproximadamente (2.2), (2.3), ou (2.4), ou resolver aproximadamente alguma outra caracterização equivalente da trajetória central. Também, pode-se escolher várias maneiras para resolver aproximadamente o sistema de equações não linear (o método de Newton é uma escolha óbvia. como são os métodos preditor-corrector, métodos do gradiente conjugado pré-condicionado, etc.). Por último, há uma liberdade considerável em desenvolver uma estratégia de reduzir o gap de dualidade (ou o parâmetro de barreira $\mu$ ) a cada iteração. (Por exemplo, encolher o gap de dualidade agressivamente parece uma idéia boa, mas também aumenta o número de iterações do método de Newton, ou outro método, que é usado para solucionar aproximadamente o novo sistema de equações não lineares). 
Capitulo 3 - Problema de Otimização Linear Canalizado

\subsection{Introdução}

Neste capítulo são apresentados o problema primal de otimização linear na forma canalizada, em seguida o problema dual e o método dual simplex com busca unidimensional. Na última seção, são apresentadas algumas extensões da busca unidimensional.

\subsection{O Problema de Otimização Linear Canalizado}

A classe de problemas (1.1) de otimização linear com restrições canalizadas é de grande interesse prático, pois representam vários problcmas reais, tais como problemas de mistura, planejamento, etc., cujas restrições, ou partc delas, são definidas por limitantes tanto superiores como inferiores, decorrentes de tolcrâncias de especificações técnicas, demanda, etc.

\subsection{O Problema Primal}

O problema primal de otimização linear com restrições canalizadas (ou, forma geral. conforme Vanderbci, 1997) é definido por: 


$$
\begin{aligned}
& \text { Minimizar } f(x)=c^{\top} x \\
& \text { Sujeito a: } d \leq A x \leq e, \quad \text { (primal) }
\end{aligned}
$$

cm que $\boldsymbol{A} \in R^{n \times n} ; \boldsymbol{d}, \boldsymbol{e} \in R^{m}$ com $d_{i} \leq e_{i} \quad i-1, \ldots, m ; c, x \in R^{n} . \Lambda$ s restrições de igualdade são representadas por $d_{i}=e_{i}$, restrições do tipo maior ou igual com $e_{i}=\infty \mathrm{c}$ do tipo menor igual com $d_{i}=-\infty$.

Proposição 3.1: Suporemos, sem perda de gencralidade, que posto $(\boldsymbol{A})=n$, isto é, suas colunas formam um conjunto de vetores de $R^{m}$ linearmente independente. $\Lambda$ dependência linear indicaria casos triviais de inexistência de soluções ótimas $(f(x) \rightarrow-\infty$, admitindo-se que o problema seja factível), ou que o problema independe da variável cuja coluna é combinação linear das demais.

Vejamos. Suponha que a n-ésima coluna seja combinação linear das demais: $\boldsymbol{a}_{n}-\sum_{j=1}^{n-1} \gamma_{j} \boldsymbol{a}_{j}$. Considere a matriz elementar

$$
\boldsymbol{E}=\left(\begin{array}{cccc}
1 & & & -\gamma_{1} \\
& \ddots & & \vdots \\
& & 1 & -\gamma_{n-1} \\
& & & 1
\end{array}\right)
$$

Note que a matriz $A E$ tem as $n-1$ primeiras colunas iguais às $n-1$ primeiras colunas da matriz $\boldsymbol{A}$ e a última coluna é nula. Assim, considerando a identidade: $A \boldsymbol{x}=(A E)\left(E^{-1} x\right)$ e, com a mudança de variável $\boldsymbol{y}=E^{-1} \boldsymbol{x}$, as restrições em (3.1) independem de $y_{n}$. Isto corresponde a fazer uma mudança de variáveis: $x_{i}=y_{i}-\gamma_{i} y_{n}, i=1, \cdots, n-1$ e $x_{n}=y_{n}$. Com esta mudança de variável, a função objetivo $f(\boldsymbol{x})-\boldsymbol{c}^{\mathrm{T}} \boldsymbol{x}=\left(\boldsymbol{c}^{\mathrm{T}} \boldsymbol{E}\right) \boldsymbol{y}$ tem a coordenada de $y_{n}$ dada por: $-\sum_{j=1}^{n-1} \gamma_{j} \mathrm{c}_{j}+\mathrm{c}_{n}$, que se for nula, então $y_{n}$ pode assumir qualquer valor em toda solução ótima, ou se for não nula, então $f(x) \rightarrow-\infty$.

Observe que o problema (1) contempla restrições que tipicamente ocorrem na prática, como por exemplo, a condição de não negatividade ou canalização das variáveis. Consideramos, sem perda de generalidade, que as variáveis sejam canalizadas, isto é, existam restrições do tipo: $d_{i} \leq x_{i} \leq e_{i}$, de modo que a matriz $A$ contém una matriz identidade $n \times n$ e, portanto, posto $(A)-n$. Observe também que restrições de igualdade são representadas no forma geral (3.1) por: $d_{i}-e_{i}$. 
O problema (3.1) pode ainda ser rescrito no forma padrão (com variáveis canalizadas), por se definir $\boldsymbol{y}=\boldsymbol{A} \boldsymbol{x}$, e então (3.1) é equivalente a:

$$
\begin{aligned}
\text { Minimizar } f(x) & =\boldsymbol{c}^{\mathrm{T}} \boldsymbol{x} \\
\text { Sujeito a: } \boldsymbol{A} \boldsymbol{x}-\boldsymbol{y} & =\boldsymbol{0} \\
\boldsymbol{d} \leq \boldsymbol{y} & \leq \boldsymbol{e} .
\end{aligned}
$$

A forma equivalente (3.2) será empregado na construção do problema dual lagrangiano a seguir.

\subsection{O Problema Dual}

Podemos determinar o problema dual de (3.1) usando o problema equivalente (3.2). Para isto definimos a função lagrangiana:

$$
\begin{aligned}
& L(\boldsymbol{x}, \boldsymbol{y}, \lambda)=\boldsymbol{c}^{\mathrm{T}} \boldsymbol{x}+\lambda^{\mathrm{T}}(\boldsymbol{y}-\boldsymbol{A} \boldsymbol{x}) \\
& L(\boldsymbol{x}, \boldsymbol{y}, \lambda)=\left(c^{\mathrm{T}}-\lambda^{\mathrm{T}} \boldsymbol{A}\right) \boldsymbol{x}+\lambda^{\mathrm{T}} \boldsymbol{y}, \quad \lambda \in R^{m} .
\end{aligned}
$$

Considere o problema Langrangiano:

$$
h(\lambda)=\min _{\langle x, y}, L(x, y, \lambda), \text { sujeito } a d \leq y \leq e .
$$

Segue diretamente da definição de (3.4) a clássica desigualdade: $f(x) \geq h(\lambda)$, para todo $\boldsymbol{x}$ factivel $\left(\boldsymbol{y}-\boldsymbol{A} \boldsymbol{x}=\boldsymbol{0}\right.$, em que $\left.L(\boldsymbol{x}, \boldsymbol{y}, \lambda)=\boldsymbol{c}^{\mathrm{T}} \boldsymbol{x}\right)$ e para todo $\lambda \in R^{m}$. Ou seja, $h(\lambda)$ fornece um limitante inferior para $f(x)$. Esta desigualdade motiva a definição do chamado problema dual lagrangiano, ou simplesmente problema dual, que consiste em determinar o melhor limitante inferior: 
Maximizar $h(\lambda), \lambda \in R^{m}$.

Note que $\lambda$ qualquer. como definido até agora, pode levar a limitantes inferiores triviais e sem interesse, isto é: $h(\lambda)=-\infty$. Assim, restringimos $\lambda$ tal que o mínimo $\mathrm{cm}$ (3.4) exista. Como $x$ é irrestrito de sinal e $y$ é canalizado, analisando (3.3), devemos escolher $\lambda$ tal que:

$$
c^{\mathrm{T}}-\lambda^{\mathrm{T}} \boldsymbol{A}=\boldsymbol{0} \text { ou } \boldsymbol{A}^{\mathrm{T}} \lambda=\boldsymbol{c} .
$$

Com $\lambda$ satisfazendo o sistema de equações lineares em (3.5), é possível expressar $h(\lambda)$ por se resolver o problema de minimização em (3.4). Usando a restrição (3.5) em (3.3), temos:

$$
L(x, y, \lambda)=\lambda^{\mathrm{T}} y=\sum_{i=1}^{m} \lambda_{i} y_{i}
$$

Então,

$$
h(\lambda)=\min L(x, y, \lambda)=\sum_{i=1}^{m} \min _{d_{i} \leq y_{i} \leq e_{i}} \lambda_{i} y_{i}=\sum_{i / \lambda_{i}>0} \lambda_{i} d_{i}+\sum_{i / \lambda_{i}<0} \lambda_{i} e_{i} .
$$

A soma de cada mínimo na expressão acima é válida, pois as variáveis $y_{i}$ são independentes. Se $\lambda_{i}=0$, então $y_{i}$ pode assumir qualquer valor no intervalo $\left[\begin{array}{ll}d_{i} & e_{i}\end{array}\right]$.

Assim, podemos explicitar a função objetivo dual como sendo:

$$
h(\lambda)=\sum_{i=1}^{m} h_{i}\left(\lambda_{i}\right)
$$

em que

$$
h_{i}\left(\lambda_{i}\right)= \begin{cases}e_{i} \lambda_{i} & \text { se } \lambda_{i} \leq 0 \\ d_{i} \lambda_{i} & \text { se } \lambda_{i} \geq 0 .\end{cases}
$$

Ou seja, o problema dual é um problema de otimização cuja a função objetivo é côncava linear por partes ilustrada na Figura 3.1. 


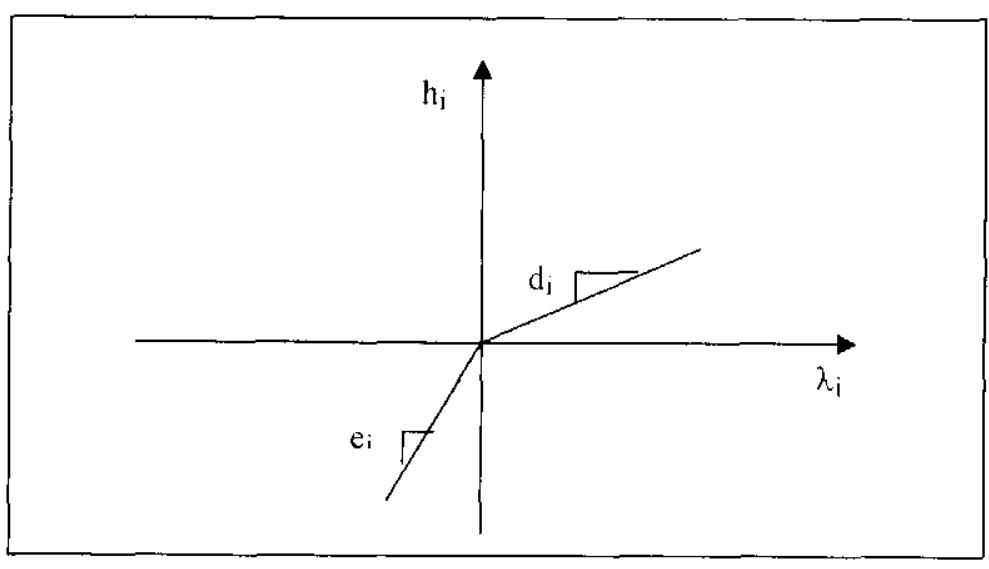

Figura 3.1- Função objetivo dual linear por partes

Desta forma, tendo resolvido o problema de minimização que surge na definição da função dual (3.4), podemos explicitar o problema dual:

$$
\text { Maximizar } h(\lambda)=\sum_{i=1}^{m} h_{i}\left(\lambda_{i}\right)
$$$$
\text { (dual) }
$$

Sujeito a: $A^{\mathrm{T}} \lambda=c$.

\subsection{A Estratégia Dual Simplex}

O problema dual sempre será factível, pois o posto $(\boldsymbol{A})=n \mathrm{e} \lambda$ irrestrito de sinal. Portanto, se o problema dual tiver solução ótima, então existirá uma solução básica ótima.

$\Lambda$ definição de uma solução básica para um problema de otimização linear por partes, com a função objetivo separável como em (3.6), é uma simples extensão da definição de solução básica da otimização linear, bastando que as variáveis não básicas sejam fixadas $\mathrm{cm}$ pontos de nãodiferenciação (Cavichia e Arenales, 2000). No caso particular da função dual em (3.6), cada função $h_{i}$ é não diferenciável apenas em $\lambda_{i}=0$. Enquanto uma solução básica factível é um vértice da região das soluções factíveis de um problema de otimização linear, uma solução básica de um problema de otimização linear por partes pode pertencer ao interior relativo da região das soluções factíveis, de modo que a solução ótima pode ser um ponto interior. Em particular, a região das soluções factíveis de (3.6) consiste num subespaço afim de $\mathrm{R}^{m}$ (subespaço transladado) e, portanto, não tem vértices

Para a construção de uma solução básica para o problema (3.6), considere uma partição básica qualquer nas colunas de $\boldsymbol{A}^{\mathrm{T}}: \boldsymbol{A}^{\mathrm{T}}=\left(\boldsymbol{B}^{\mathrm{T}}, \boldsymbol{N}^{\mathrm{T}}\right)$ (i.e., selecione uma sub-matriz invertível $n \times n$ de $\boldsymbol{A}^{\mathrm{T}}$, denotada por $\boldsymbol{B}^{\mathrm{T}}$, e denote por $\boldsymbol{N}^{\mathrm{T}}$ a sub-matriz $n \times(m-n)$ formada pelas colunas restantes de $\boldsymbol{A}^{\mathrm{T}}$ ). 
Esta partiçâo nas colunas de $A^{\mathrm{T}}$ introduz uma partição no vetor de variáveis: $\lambda=\left(\lambda_{B}, \lambda_{1}\right)$ ). $O$ i-ésimo elemento de $\lambda_{B}$ é denotado por $\lambda_{B_{i}}$ (ou, também: $\lambda_{i}, i \in B$ ). $\Lambda$ i-ésima coluna de $\boldsymbol{B}^{\top}$ é denotada por $a_{B_{i}}^{T}$ e a q-ésima coluna de $N^{T}$ é denotada por $a_{N_{q}}^{T}$.

Assim, podemos escrever a soluçâo geral do sistema de equações em (3.5):

$$
\boldsymbol{A}^{\mathrm{T}} \lambda=c \Leftrightarrow \boldsymbol{B}^{\mathrm{T}} \lambda_{B}+N^{\mathrm{T}} \hat{\lambda}_{N}=c \Leftrightarrow \lambda_{B}=\left(\boldsymbol{B}^{\mathrm{T}}\right)^{-1} c-\left(\boldsymbol{B}^{\mathrm{T}}\right)^{-1} N^{\mathrm{T}} \lambda_{N}
$$

Uma solução particular, chamada solução básica dual, associada à partição básica é dada por:

$$
\hat{\lambda}=\left[\begin{array}{l}
\hat{\lambda}_{B} \\
\hat{\lambda}_{N}
\end{array}\right], \text { cm que } \hat{\lambda}_{h}^{\mathrm{T}}=c^{\mathrm{T}} \mathrm{B}^{-1} \mathrm{e} \hat{\hat{\lambda}}_{k}^{\mathrm{T}}=0
$$

A soluçâo definida $\mathrm{cm}$ (3.8), independentemente da partição básica, satisfaz as restrições do problema dual e, portanto, é chamada dual factivel.

Para a solução básica dual, $\hat{\lambda}_{\mathrm{B}}$ pode ser positivo ou negativo (se nulo, é o caso de degeneração dual) e, portanto, para a avaliação da função dual $h(\lambda)$ o valor de $y_{B_{t}}$ fica determinado por:

$$
\hat{y}_{B_{i}}=\left\{\begin{array}{lll}
d_{B_{i}} & \text { se } & \hat{\lambda}_{B_{1}} \geq 0 \\
\mathcal{e}_{B_{1}} & \text { se } & \hat{\lambda}_{B_{1}} \leq 0 .
\end{array}\right.
$$

Em caso de degeneração da solução dual, i.e., $\hat{\hat{\lambda}}_{B_{1}}=0, y_{B_{i}}$ pode, a principio, assumir qualquer valor no intervalo $\left\{d_{B_{i}} e_{B_{i}}\right]$ que. por conveniência, escolheremos $\hat{y}_{B_{i}}=d_{B_{i}}$ ou $\hat{y}_{B_{i}}=e_{B_{i}}$. Deste modo, se escolhermos $\hat{y}_{B_{1}}=d_{B_{1}}$, então para efeito de desenvolvimentos futuros, a variável $\lambda$ é vista como positiva (apesar de ter seu valor nulo). Assim, se a variável $\lambda_{B_{j}}$ sofre um acréscimo, $\hat{y}_{B_{i}}$ não se altera conforme (3.9); caso contrário, um decréscimo em $\lambda_{B_{t}}$ tomaria a variável negativa e, portanto, $\hat{y}_{B_{1}}$ deveria ser $e_{B_{i}}$. 
Observe também que a partição básica fornece uma partição nas equações de (3.2):

$$
\left[\begin{array}{l}
\boldsymbol{B} \\
N
\end{array}\right] \boldsymbol{x}=\left[\begin{array}{l}
y_{B} \\
y_{N}
\end{array}\right] \Leftrightarrow\left\{\begin{array}{l}
\boldsymbol{B} \boldsymbol{x}=\boldsymbol{y}_{B} \\
N \boldsymbol{x}=\boldsymbol{y}_{N}
\end{array}\right.
$$

Da primeira cquação acima, com $y_{B}=\hat{\boldsymbol{y}}_{B}$, define a solução básica primal

$$
\hat{\boldsymbol{x}}=\boldsymbol{B}^{-1} \hat{y}_{B}
$$

Além disso, a segunda equação define o valor de $y_{N}$, de modo que as restrições $\boldsymbol{y}=\boldsymbol{A} \boldsymbol{x}$ (exceto as canalizaçôes) do problema primal estão satisfeitas:

$$
\hat{\boldsymbol{y}}_{\lambda}=N B^{-1} \hat{y}_{B} \text {. }
$$

Assim, a solução $(\hat{\boldsymbol{x}}, \hat{\boldsymbol{y}})$ dada por (3.9)-(3.11) satisfaz as restrições $\boldsymbol{A} \boldsymbol{x}=\boldsymbol{y}$ e $\boldsymbol{d}_{B} \leq \boldsymbol{y}_{B} \leq \boldsymbol{e}_{B}$, enquanto que as restrições $d_{N} \leq y_{N} \leq \boldsymbol{e}_{N}$ podem estar violadas.

Definição 3.1: Considere uma partição básica sobre as colunas de $\boldsymbol{A}^{\mathrm{T}}$. As soluções em (3.8) e (3.9)-(3.11) são chamadas soluções básicas primal dual e primal, respectivamentc, associadas à partição básica. Se $\boldsymbol{d}_{N} \leq \hat{\boldsymbol{y}}_{N} \leq \boldsymbol{e}_{N}$, então a solução básica primal é factível, ou simplesmente, primal factível.

Para o par de soluções básicas, dual e primal, associadas a uma partição básica, temos: $h(\hat{\lambda})=f(\hat{x})$, pois:

$$
h(\hat{\lambda})=\sum_{i=1}^{m} h_{i}\left(\hat{\lambda}_{i}\right)=\sum_{i \in B} h_{i}\left(\hat{\lambda}_{i}\right)+\sum_{i \in N} h_{i}\left(\hat{\lambda}_{i}\right)=\sum_{i \in B} h_{i}\left(\hat{\lambda}_{i}\right)=\hat{\lambda}_{B}^{T} y_{B}=c^{\mathrm{T}} \boldsymbol{B}^{-1} \hat{\boldsymbol{y}}_{B}=\boldsymbol{c}^{\mathrm{T}} \hat{\boldsymbol{x}}=f(\hat{\boldsymbol{x}}) .
$$

Como $h(\hat{\lambda})$ é um limitante inferior para $f(x)$, segue o seguinte teorema. 
Teorema 3.1: Considere uma partição básica qualquer e as soluções básicas primal e dual associadas. Se a solução for factivel (i.e.., $\boldsymbol{d}_{N} \leq \hat{\boldsymbol{y}}_{N} \leq \boldsymbol{e}_{N}$ ) então as soluções básicas primal e dual são soluções ótimas dos problemas primal e dual respectivamente.

Em geral, para a resolução de um problema de otimização linear qualquer usando-se o método simplex, se faz necessária a fase I, que consiste em outro problema de otimização linear a ser resolvido. Entretanto. o método simplex aplicado ao problema dual (3.6) não requer a fase l e pode ser iniciado a partir de qualquer partição básica, a qual fornece sempre uma solução dual factivel, conforme (3.8).

Como é comum em problemas práticos, restrições do tipo: $\boldsymbol{l} \leq \boldsymbol{x} \leq \boldsymbol{u}$ (implicitamente considcradas em $\boldsymbol{d} \leq \boldsymbol{A} \boldsymbol{x} \leq \boldsymbol{e}$ ) fornecem uma partição óbvia com $\boldsymbol{B}=\boldsymbol{I}$. Caso não haja limitantes naturais para uma variável $x_{i}$, pode-se considerar $l_{i}=-\mathrm{M}$, ou $u_{i}=\mathrm{M}$, com $M$ é um valor suficientemente grande e a partição básica inicial segue válida.

Considere uma partição básica e suas soluções básicas associadas (3.8) e (3.9)-(3.11). Se as condições do teorema 3.1 não são satisfeitas, perturbamos a solução básica dual, de modo a aumentar a função objetivo dual $h(\lambda)$

$\Lambda$ estratégia dual simplex consiste na perturbação de apenas uma variável dual não básica (uma componente de $\lambda_{N}$ ). Temos dois casos a considerar. Por simplicidade, supomos a solução dual não degenerada, caso contrário, $\hat{\lambda}_{B_{1}}=0$ para algum $i \in B$.

Caso a) A variável $\lambda_{v_{y}}$ é acrescida: $\left\{\begin{array}{l}\lambda_{N_{q}}=\delta, \text { com } \delta \geq 0 \text { e pequeno } \\ \lambda_{v_{1}}=0, \quad i=1, \ldots, m-n, i \neq q .\end{array}\right.$

Ou seja:

$$
\lambda_{N}=\delta \tilde{\boldsymbol{e}}_{q},
$$

em que $\tilde{\boldsymbol{e}}_{q}$ é a $q$-ésima coluna da matriz identidade $(m-n) \times(m-n)$.

Com esta perturbação nas variáveis não básicas, devemos determinar a perturbação nas variáveis básicas de modo que o sistema $A^{\top} \lambda=c$ seja satisfeito. Para isto, substituindo (3.12) $\mathrm{cm}$ (3.7) segue: 


$$
\lambda_{B b}=\left(\boldsymbol{B}^{\mathrm{T}}\right)^{-1} c-\delta\left(\boldsymbol{B}^{\mathrm{T}}\right)^{-1} \mathbf{a}_{N_{q}}^{\mathrm{T}}
$$

De (3.8), tcmos que:

$$
\lambda_{B}=\hat{\lambda}_{B}+\delta \boldsymbol{\eta}_{B},
$$

com $\boldsymbol{\eta}_{\beta}=-\left(\boldsymbol{B}^{\mathrm{T}}\right)^{-1} \boldsymbol{a}_{N_{4}}^{T}$ é o vetor das componentes básicas da direção dual simplex.

Portanto a nova solução dual pode ser escrita por:

$$
\lambda=\left(\begin{array}{l}
\lambda_{B} \\
\lambda_{N}
\end{array}\right)=\left(\begin{array}{l}
\hat{\lambda}_{B} \\
\hat{\lambda}_{N}
\end{array}\right)+\delta\left(\begin{array}{cc}
-\left(\boldsymbol{B}^{\mathrm{T}}\right)^{-1} & \boldsymbol{a}_{N_{q}}^{\mathrm{T}} \\
\tilde{\boldsymbol{e}}_{q}
\end{array}\right)
$$

ou ainda,

$$
\lambda=\hat{\lambda}+\delta \eta
$$

em que $\boldsymbol{\eta}=\left(\begin{array}{cc}-\left(\boldsymbol{B}^{\mathrm{T}}\right)^{-1} & \boldsymbol{a}_{N_{q}}^{\mathrm{T}} \\ \tilde{\boldsymbol{e}}_{q} & \end{array}\right)$ é chamada direção dual simplex.

Escrevendo a função objetivo dual para a nova solução (3.14), considerando $\delta$ suficientemente pequeno, de modo que a função $h(\lambda)$ é linear e nenhuma variável dual troca de sinal, temos:

$$
h(\lambda)=h(\hat{\lambda}+\delta \boldsymbol{\eta})=\sum_{i=1}^{n} h_{B_{i}}\left(\hat{\lambda}_{B_{i}}+\delta \eta_{B_{i}}\right)+\sum_{i=1}^{m-n} h_{N_{1}}\left(\hat{\lambda}_{N_{1}}+\delta \eta_{N_{i}}\right)
$$

Como supomos a solução básica não degenerada, i.e., $\hat{\lambda}_{B_{\triangleleft}} \neq 0, i=1, \ldots, n$ e lembrando que apenas a q-ésima variável não básica foi perturbada: $\lambda_{N_{q}}=\delta \geq 0$ (portanto o coeficiente de $\lambda_{N_{q}}$ na função dual é $d_{N_{q}}$, veja Figura 3.1), segue:

$$
\begin{aligned}
& h(\lambda)=h(\hat{\lambda}+\delta \boldsymbol{\eta})=\sum_{i-1}^{n} \hat{y}_{B_{i}}\left(\hat{\lambda}_{B_{i}}+\delta \eta_{B_{i}}\right)+\delta d_{N_{k}}=\sum_{i=1}^{n} \hat{y}_{B_{i}} \hat{\lambda}_{B_{i}}+\delta\left(\sum_{i=1}^{n} \hat{y}_{B_{i}} \eta_{B_{i}}+d_{N_{k}}\right) \\
& h(\lambda)=h(\hat{\lambda})+\delta\left(\hat{y}_{B}^{\top} \boldsymbol{\eta}_{B}+d_{N_{k}}\right)
\end{aligned}
$$


Substituindo na expressão acima as componentes básicas da direçẫo dual simplex dadas $\mathrm{cm}$ (3.13) e a solução básica primal em (3.10), vem que:

$$
\begin{aligned}
& h(\lambda)=h(\hat{\hat{\lambda}})+\delta\left(\hat{\boldsymbol{y}}_{B}^{\mathrm{T}}\left(-\boldsymbol{B}^{\mathrm{T}}\right)^{-1} \boldsymbol{a}_{N_{q}}+d_{N_{4}}\right) \\
& h(\lambda)=h(\hat{\hat{\lambda}})+\delta\left(-\boldsymbol{a}_{N_{\mathrm{K}}}^{\mathrm{T}} \hat{\boldsymbol{x}}+d_{N_{q}}\right) .
\end{aligned}
$$

Em resumo, a perturbação da solução básica dual na direção dual simplex dada por (3.14), decorrente da estratégia dual simplex (3.12), promove uma variação da função objetivo dual dada por (3.15).

Portanto, se $d_{N_{K}}-\boldsymbol{a}_{N_{k}}^{\mathrm{T}} \hat{\boldsymbol{x}}>0$, ou seja, se o limite inferior da $k$-ésima restrição primal for violado, a dirę̧ão dual simplex é uma direção de subida.

A expressão (3.15) é valida, desde que $\lambda_{B}$ em (3.13) não sofra mudança de sinal, pois caso contrário, o coeficiente da função objetivo dual é alterado. Se $\hat{\lambda}_{B_{1}}$ c $\eta_{B_{i}}$ têm sinais opostos. então $\lambda_{B_{1}}$ pode trocar de sinal com o crescimento de $\delta$. Portanto temos dois casos a considerar:

Caso (i) Se $\hat{\lambda}_{B_{i}}<0$ e $\eta_{B_{1}}>0$, então impondo que: $\hat{\lambda}_{B_{i}}+\delta \eta_{B_{i}} \leq 0$, segue que: $\delta \leq-\frac{\hat{\lambda}_{B_{i}}}{\eta_{B_{t}}}$

Caso (ii) Se $\hat{\lambda}_{B_{t}}>0$ e $\eta_{B_{i}}<0$, então impondo que: $\hat{\lambda}_{B_{r}}+\delta \eta_{B_{i}} \geq 0$, segue que: $\delta \leq-\frac{\hat{\lambda}_{B_{i}}}{\eta_{B_{i}}}$.

Assim, para que não haja mudança de sinal em $\lambda_{B_{1}}$ devemos ter:

$$
\delta \leq \delta_{i}=-\frac{\hat{\lambda}_{B_{i}}}{\eta_{B_{i}}} \text {, para todo } i=l \ldots . . n \text {, tal que } \hat{\lambda}_{B_{i}} \text { c } \eta_{B_{i}} \text { tenham sinais opostos. }
$$

Seja $p_{0}$ um índice básico tal que:

$$
\delta_{p_{11}}=-\frac{\hat{\lambda}_{B_{P_{0}}}}{\eta_{B_{P_{0}}}}=\min \left\{-\frac{\hat{\lambda}_{B_{i}}}{\eta_{B_{i}}}, \text { tal que }:-\frac{\hat{\lambda}_{B_{i}}}{\eta_{B_{i}}} \geq 0, i=1, \ldots . n\right\}
$$


Para $0 \leq \delta \leq \delta_{P_{0}}$ temos que: $h(\lambda)=h(\hat{\lambda})+\delta\left(d_{y_{1}}-\hat{y}_{N_{1}}\right)$. Quando $\delta=\delta_{P_{0}}$ temos de (3.13) e (3.16) que $p_{i}$-ésima restrição básica torna-se nula: $\lambda_{B_{l_{0}}}=0$, o que sugere uma redefinição da partição básica, onde $\lambda_{B_{p_{0}}}$ torna-se não básica e $\lambda_{N_{q}}=\delta_{P_{*}}>0$ torna-se básica. A Figura 2 ilustra a situação.

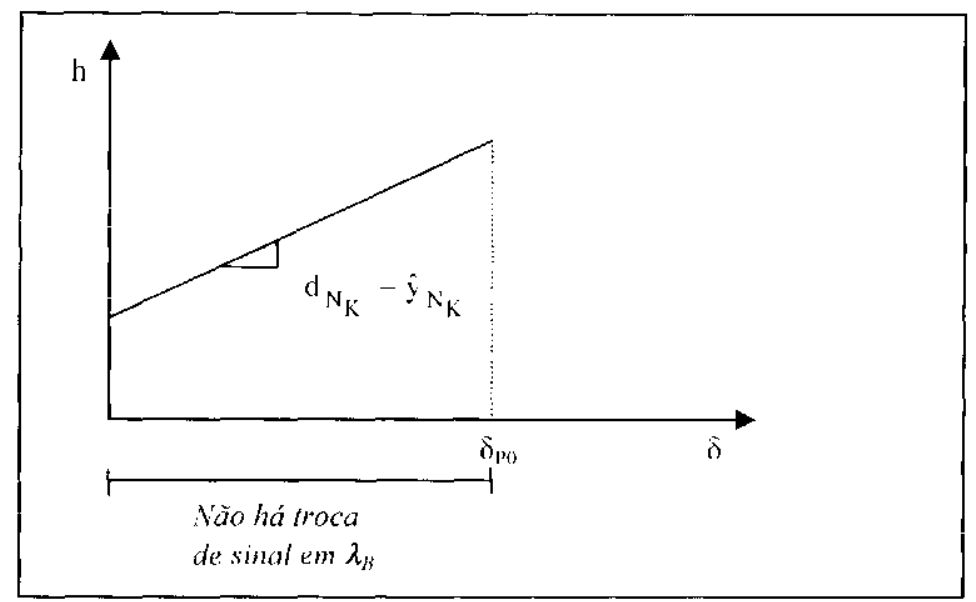

Figura 3.2-Gráfico da função dual no intervalo $\left[0, \delta_{r^{\prime}}\right]$

Obviamente, como as variáveis duais são irrestritas de sinal, o crescimento de $\delta$ não precisa ser limitado por $h(\hat{\lambda})$. Para valores de $\delta>\delta_{P_{0}}$, a expressão da função dual se altera, mas a direção dual pode ainda ser de subida. Isto sugere uma busca na direção $\eta$ para a escolha de $\delta$. Esta busca ć linear por partes, devido à característica da função objetivo dual.

Considere que: $h(\lambda)=h(\hat{\lambda})+\delta h_{0}, \mathrm{~cm}$ que $h_{01}=\left(d_{N_{4}}-\hat{y}_{N_{4}}\right)$.

Se dermos um pequeno acréscimo ao valor de $\delta_{P_{0}}$, ou seja, $\delta=\delta_{P_{0}}+\varepsilon$, em que $\varepsilon>0 \mathrm{c}$ pequeno, então $\lambda_{B_{r_{0}}}$ troca de sinal. (Observe que $\hat{\lambda}_{B_{p_{0}}}=0$ para $\delta=\delta_{P_{0}}$ ).

Analisaremos somente o caso $(i): \hat{\lambda}_{B_{P_{0}}}<0$ e $\eta_{B_{i}}>0$, o outro caso $\left(\hat{\lambda}_{B_{l_{0}}}>0\right.$ e $\left.\eta_{B_{1}}<0\right)$ segue de maneira análoga. Então, para $0<\delta<\delta_{P_{0}}$ a variável $\lambda_{B_{b_{b}}}<0$ e para $\delta>\delta_{P_{G}}$, a variável $\lambda_{B_{b_{b}}}>0$. Substituindo $\delta=\delta_{p_{0}}+\varepsilon$ em (3.14) temos: 


$$
\begin{aligned}
& \lambda=\hat{\lambda}+\left(\delta_{p_{1}}+\varepsilon\right) \eta \\
& \lambda=\left(\hat{\lambda}+\delta_{P_{0}} \eta\right)+\varepsilon \eta \\
& \lambda=\lambda^{1}+\varepsilon \eta,
\end{aligned}
$$

em que $\lambda^{\prime}=\hat{\lambda}+\delta_{p_{0}} \boldsymbol{\eta}$. Observe que $\lambda_{N_{4}}=\delta_{p_{i,}}+\varepsilon$ e $\lambda_{N_{i}}=0, i \neq q$.

Assim, a função objetivo dual pode ser expressa por:

$$
\begin{aligned}
& h(\lambda)=\sum_{i=1}^{n} h_{B_{i}}\left(\hat{\lambda}_{B_{i}}\right)+\sum_{i=1}^{m-n} h_{N_{i}}\left(\hat{\lambda}_{N_{i}}\right) \\
& h(\lambda)=\sum_{i=1}^{n} h_{B_{i}}\left(\lambda_{B_{i}}^{1}+\varepsilon \eta_{B_{i}}\right)+d_{N_{k}}\left(\delta_{p_{0}}+\varepsilon\right) .
\end{aligned}
$$

Suporemos por simplicidade que $\lambda_{R_{1}}^{1} \neq 0, i \neq p_{\theta}$, ou seja. que o mínimo em (3.16) ocorre somente para o índice $p_{0}$, apenas a variável $\lambda_{\beta_{p_{1}}}$ se anula com $\delta=\delta_{p_{01}}$.

Lembrando que $\lambda_{B_{p_{0}}}^{1}=\hat{\lambda}_{B_{P_{0}}}+\delta_{P_{0}} \eta_{B_{l_{0}}}=0 \mathrm{e} \varepsilon>0$ e pequeno, temos que:

$$
h(\lambda)=\sum_{\substack{i=1 \\ i \neq p_{i}}}^{n} \hat{y}_{B_{i}}\left(\hat{\lambda}_{B_{i}}^{1}+\varepsilon \eta_{B_{i}}\right)+h_{B_{p_{0}, 0}}\left(\varepsilon \eta_{B_{p_{0}}}\right)+\left(\delta_{p 0}+\varepsilon\right) d_{N_{4}}
$$

Como $\eta_{B_{p_{0}}}>0$, pois estamos analisando o caso (i), temos que $h_{B_{p_{0}}}\left(\varepsilon \eta_{B_{p_{0}}}\right)=\varepsilon d_{B_{p_{0}}} \eta_{B_{p_{0}}}$. Logo,

$$
h(\lambda)=\sum_{i=1}^{n} \hat{y}_{B_{i}}\left(\lambda_{B_{i}}^{1}\right)+\delta_{p_{0}} d_{N_{q}}+\varepsilon\left(\sum_{\substack{i=1 \\ i \neq p_{0}}}^{n} y_{B_{i}} \eta_{B_{i}}+d_{B_{b_{b}}} \eta_{B_{b_{0}}}+d_{N_{q}}\right)
$$

As seguintes igualdades são asseguradas: 


$$
\begin{aligned}
& \text { I) } h\left(\lambda^{1}\right)=h\left(\hat{\dot{\lambda}}+\delta_{P_{i}} \eta\right)=\sum_{i=1}^{n} h_{B_{i}}\left(\hat{\lambda}_{B_{i}}+\delta_{P_{o}} \eta_{B_{i}}\right)+\sum_{i=1}^{m-n} h_{N_{i}}\left(\hat{\lambda}_{N_{i}}+\delta_{P_{0}} \eta_{N_{i}}\right) \\
& h\left(\lambda_{i}^{1}\right)=\sum_{i=1}^{n} h_{B_{i}}\left(\lambda_{B_{i}}^{1}\right)+\delta_{p_{0}} d_{N_{q}} ;
\end{aligned}
$$

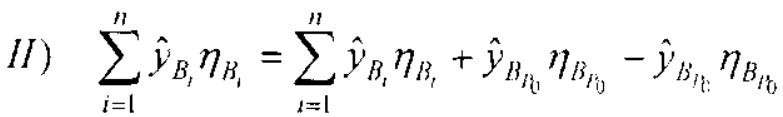

$$
\begin{aligned}
& \sum_{\substack{i=1 \\
i \neq p_{i}}}^{n} \hat{y}_{B_{1}} \eta_{B_{i}}=\sum_{i=1}^{n} \hat{y}_{B_{1}} \eta_{B_{i}}-\mathfrak{e}_{B_{\beta_{0}}} \eta_{B_{B_{b}}} \\
& \text { III) } \sum_{i=1}^{n} \hat{y}_{B_{1}} \eta_{B_{i}}=\hat{\boldsymbol{y}}_{B}^{T} \boldsymbol{\eta}_{B}=\hat{\boldsymbol{y}}_{B}^{\prime}\left(-\left(\boldsymbol{B}^{\mathrm{T}}\right)^{\prime} \boldsymbol{a}_{N_{N_{4}}}\right)=-\mathbf{a}_{N_{4}}^{\mathrm{T}}\left(\boldsymbol{B}^{-1} \hat{\boldsymbol{y}}_{B}\right)=-\boldsymbol{a}_{N_{4}}^{\mathrm{T}} \hat{\boldsymbol{x}} .
\end{aligned}
$$

Portanto, a expressão (3.17) pode ser reescrita usando $(I),(I I)$ e $(I I)$ :

$$
h(\lambda)=h\left(\lambda^{i}\right)+\varepsilon\left(h_{0}-\left(e_{B_{h_{1}}}-d_{B_{h_{0}}}\right) \eta_{B_{p_{0}}}\right),
$$

em que $h_{0}=-\boldsymbol{a}_{N_{q}}^{\mathrm{T}} \hat{\boldsymbol{x}}+d_{N_{q}}$.

As expressões descritas anteriormente para a função dual são válidas desde que $\varepsilon>0 \mathrm{e}$ suficientemente pequeno. Analisaremos agora, qual o maior valor para o incremento $\varepsilon$, ou seja, determinaremos o tamanho do passo de modo que $\hat{\lambda}_{k_{s}}+\left(\delta_{p_{0}}+\varepsilon\right) \eta_{B_{q}}$ não mude de sinal, isto é, $\hat{\lambda}_{B_{i}}$ tem o mesmo sinal de $\hat{\lambda}_{B_{i}}+\left(\delta_{p_{0}}+\varepsilon\right) \eta_{B_{i}}, i \neq p_{n} . \Lambda$ ssim, os valores de $\mathcal{E}$ que promovem mudança de sinal em $\lambda_{B_{1}}$ são dados por:

$$
\varepsilon_{i}=-\frac{\hat{\lambda}_{B_{i}}+\delta_{p_{0}} \eta_{B_{i}}}{\eta_{B_{i}}}=-\frac{\hat{\lambda}_{B_{i}}}{\eta_{k_{i}}}-\delta_{p_{0}}>0, i \neq p_{0}
$$

Denominando $\delta_{i}=\varepsilon_{i}+\delta_{p_{0}}=-\frac{\hat{\lambda}_{B_{1}}}{\eta_{B_{1}}}$, o valor máximo de $\delta$ que evita a segunda mudança de sinal em $\lambda_{B_{i}}$ é dado por:

$$
\delta_{p_{1}}=-\frac{\hat{\lambda}_{B_{p_{1}}}}{\eta_{B_{p_{1}}}}=\min \left\{-\frac{\dot{\lambda}_{B_{1}}}{\eta_{B_{1}}}>0, \quad i \neq p_{0}\right\}
$$


No cálculo do índice $p_{0}$ (veja (3.16)) as mesmas razões já haviam sido calculadas e $p_{0}$ foi o índice que forneceu a menor razão. Agora $p_{l}$ é o indice da segunda menor razão. A Figura 3.3 representa o gráfico da função objetivo dual.

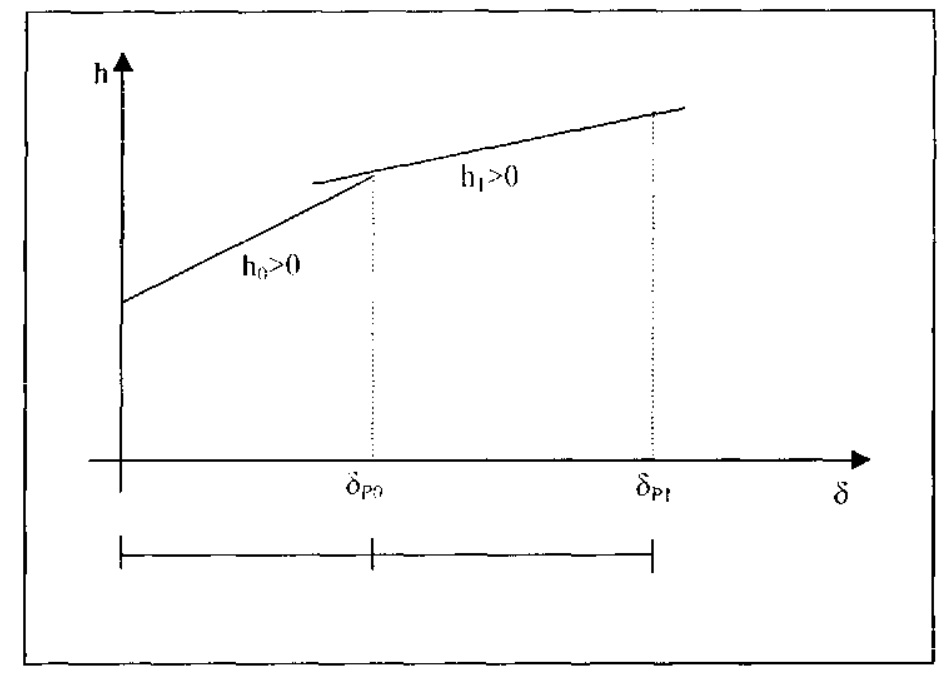

Figura 3.3 - Gráfico da função dual após a mudança de sinal de uma variável básica

Podemos examinar o passo dual além de $\delta_{p_{1}}: \delta>\delta_{p_{1}}$ de maneira análoga ao que foi feito até aqui. Em geral temos: $h_{s+1}=h_{s}-\left(e_{B_{p_{s}}}-d_{R_{p_{s}}}\right) \eta_{B_{p_{s}}}$. A Figura 3.4 ilustra a função dual e como uma busca na direção dual simplex pode ser feita para maximizar $h(\hat{\lambda}+\delta \eta)$.

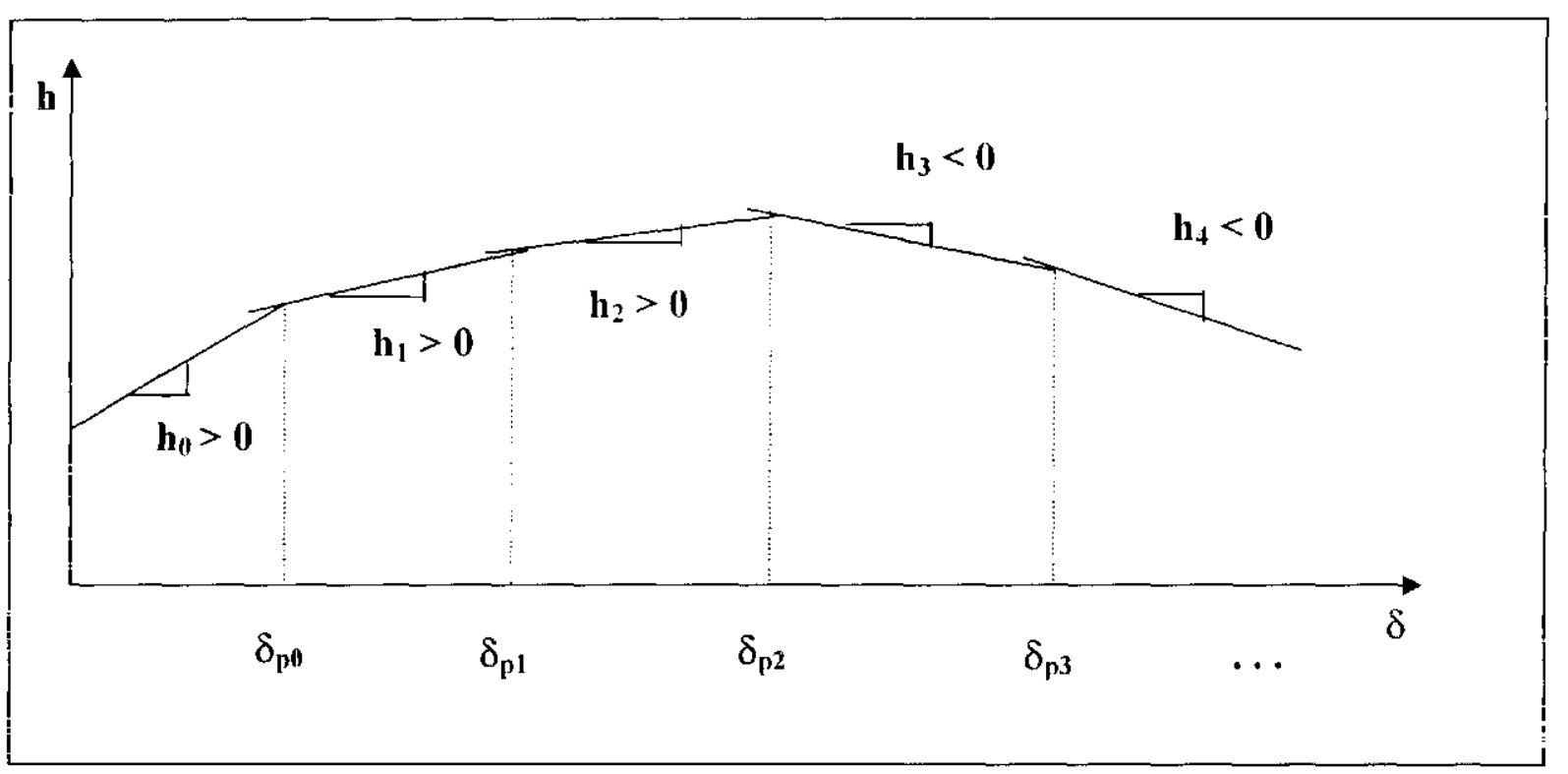

Figura 3.4 - Gráfico da função dual: $\delta_{\mathrm{p} 2}$ ć o ponto de máximo 
A busca unidimensional na direção dual simplex consiste em escolher um índice $\ell$ tal que:

$$
\mathrm{h}_{i}>0 \text { e } \mathrm{h}_{i, 1} \leq 0 \text {. }
$$

Caso b) A variável $\lambda_{N_{q}}$ é decrescida: $\left\{\begin{array}{l}\lambda_{N_{N_{q}}}=-\delta, \text { com } \delta \geq 0 \text { e pequeno } \\ \lambda_{N_{1}}=0, i=1, \ldots, m-n, i \neq q .\end{array}\right.$

Ou seja:

$$
\lambda_{N}=-\delta \tilde{\boldsymbol{e}}_{q}
$$

O procedimento é análogo ao caso a), notando agora que:

$$
\lambda_{B}=\hat{\lambda}_{B}-\delta \eta_{B}
$$

em que $\boldsymbol{\eta}_{B}=-\left(\boldsymbol{B}^{\mathrm{T}}\right)^{-1} \boldsymbol{a}_{N_{q}}^{\mathrm{T}}$ é o vetor das componentes básicas da direção dual simplex. Portanto a nova solução dual pode ser escrita por:

$$
\lambda=\left(\begin{array}{l}
\hat{\lambda}_{B} \\
\lambda_{N}
\end{array}\right)=\left(\begin{array}{l}
\hat{\lambda}_{B} \\
\hat{\lambda}_{N}
\end{array}\right)-\delta\left(\begin{array}{c}
-\left(\boldsymbol{B}^{\mathrm{T}}\right)^{-1} \boldsymbol{a}_{N_{q}}^{\mathrm{T}} \\
\tilde{\boldsymbol{e}}_{q}
\end{array}\right)
$$

ou ainda,

$$
\lambda=\hat{\lambda}-\delta \eta
$$

Fazendo o mesmo desenvolvimento da função objetivo dual para a nova solução (3.19) e considerando as devidas mudanças, segue que:

$$
h(\lambda)=h(\hat{\lambda})+\delta\left(\boldsymbol{a}_{N_{4}}^{\mathrm{T}} \hat{\boldsymbol{x}}-e_{N_{4}}\right)
$$

Portanto, se $\boldsymbol{a}_{N_{q}}^{\mathrm{T}} \hat{\boldsymbol{x}}-e_{N_{q}}>0$, ou seja, se o limite superior da $q$-ésima restrição primal for violado, então a direção dual simplex é uma direção de subida. 
A expressão (3.20) é valida desde que $\hat{\lambda}_{B}$ não sofra mudança de sinal. Logo se $\hat{\lambda}_{B}$ e $\boldsymbol{\eta}_{B}$, tem o mesmo sinal, então $\lambda_{B}$ pode trocar de sinal com o crescimento de $\delta$. Portanto, de maneira análoga ao caso a), para que não haja mudança de sinal em $\lambda_{B_{,}}$devemos ter:

$$
\delta \leq \delta_{i}=\frac{\hat{\lambda}_{B_{i}}}{\eta_{B_{1}}},
$$

seja $p_{o}$ um índice básico tal que:

$$
\delta_{p_{0}}=\frac{\hat{\lambda}_{B_{b_{0}}}}{\eta_{B_{p_{0}}}}=\min \left\{\frac{\hat{\lambda}_{B_{r}}}{\eta_{B_{i}}}, \text { tal que } \frac{\hat{\lambda}_{B_{i}}}{\eta_{B_{i}}}<0, i=1, \ldots, m\right\}
$$

Para $0 \leq \delta \leq \delta_{P_{0}}$ temos que: $h(\hat{\lambda})=h(\hat{\lambda})+\delta\left(\hat{y}_{N_{y}}-e_{N_{q}}\right)$. Quando $\delta=\delta_{P_{0}}$ temos que a $p_{\theta}$-ésima restrição básica torna-se nula, enquanto que $\lambda_{N_{q}}=-\delta \leq 0$, o que sugere uma redefinição da partição básica. $\Lambda$ Figura 3.5 ilustra a situação.

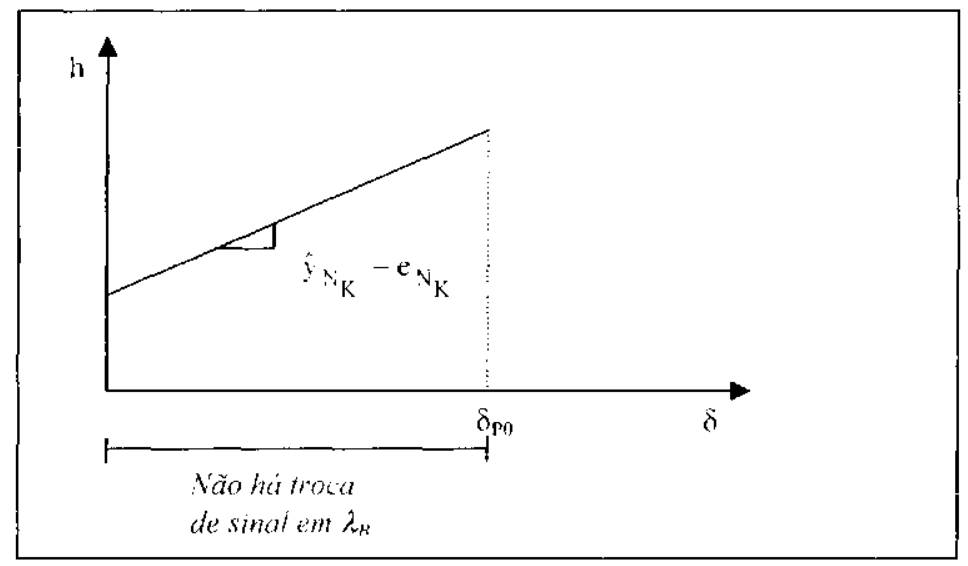

Figura 3.5 - Gráfico da função dual no intervalo $\left[0, \delta_{r_{0}}\right]$

Como já observado no caso a), o valor de $\delta$ pode ser maior do que $\delta_{P_{0}}$ pois as variáveis duais não têm restrição de sinal. Para analisar a função dual para valores além de $\delta_{P_{0}}$ consideramos $\delta=\delta_{p_{0}}+\varepsilon, \quad \varepsilon>0$ e suficientemente pequeno. Logo a nova solução dual é dada por: 


$$
\lambda=\left[\begin{array}{l}
\hat{\lambda}_{B} \\
\hat{\lambda}_{N}
\end{array}\right]-\left(\delta_{P_{0}}+\varepsilon\right)\left[\begin{array}{l}
\boldsymbol{\eta}_{B} \\
\boldsymbol{\eta}_{N}
\end{array}\right]
$$

Analogamente ao caso a), obtemos a seguinte expressão para a função dual:

$$
h(\lambda)=h\left(\hat{\lambda}-\delta_{P_{0}} \boldsymbol{\eta}\right)+\varepsilon h_{1}, \quad \text { onde } \quad h_{1}=h_{0}-\left(e_{B_{p_{0}}}-d_{B_{p_{0}}}\right) \eta_{B_{P_{0}}} \quad \text { c } \quad h_{0}=\boldsymbol{a}_{N_{4}}^{\mathrm{T}} \hat{\boldsymbol{x}}-e_{N_{4}} \text {. }
$$

A busca unidimensional é realizada da mesma forma que foi feita para o caso $(a)$. Esta busca foi considerada na implementação do algoritmo, para a qual fizemos algumas alterações, que serão apresentadas na seção 3.7 .

Embora Maros $\left(2003^{\mathrm{a}}\right)$ tenha desenvolvido o algoritmo dual simplex para problemas com variáveis canalizadas, baseada na característica da função dual (linear por partes), o autor não considera a canalização nas restrições como fizemos, explorando as particularidades deste caso.

Na próxima seção, apresentamos o algoritmo para o método dual simplex com a busca undimensional

\subsection{Algoritmo Dual Simplex com Busca Undimensional (DSBU)}

Resumimos o método dual simplex com busca unidimensional linear por partes exata especializado para problemas no formato geral dado por (3.1):

\section{Passo 0: INICIALIZAÇÃO}

Determine uma partição básica nas colunas de $\boldsymbol{A}^{\mathrm{T}}=\left(\boldsymbol{B}^{\mathrm{T}}, \boldsymbol{N}^{\mathrm{T}}\right)$.

Calcule a solução dual: $\hat{\lambda}=\left[\begin{array}{l}\hat{\lambda}_{k} \\ \hat{\lambda}_{N}\end{array}\right] \operatorname{com} \hat{\lambda}_{k}^{T}=c^{\mathrm{T}} \boldsymbol{B}^{-1}$ e $\hat{\lambda}_{N}^{\mathrm{T}}=0$.

Calcule a solução primal: $\hat{\boldsymbol{x}}=\boldsymbol{B}{ }^{\prime} \hat{\boldsymbol{y}}_{B}, \operatorname{com} \hat{y}_{B_{t}}=\left\{\begin{array}{lll}d_{B_{t}} & \text { se } & \hat{\lambda}_{B_{s}} \geq 0 \\ e_{B_{t}} & \text { se } & \hat{\lambda}_{B_{i}} \leq 0 .\end{array}\right.$

$I T=\mathbf{0}$. 


\section{Passo 1: OTIMALIDADE}

Escolha um índice $q, 1 \leq q \leq m-n$ que forncce a maior violação, ou seja,

caso a): $d_{N_{4}}-a_{N_{q}}^{\mathrm{T}} \hat{\boldsymbol{x}}>0 \quad$ (limite inferior violado), $h_{0}=d_{N_{4}}-\boldsymbol{a}_{N_{4}}^{\mathrm{T}} \hat{\boldsymbol{x}}$

ou

caso b): $\boldsymbol{a}_{N_{q}}^{\mathrm{T}} \hat{\boldsymbol{x}}-e_{N_{q}}>0$ (limite superior violado), $h_{0}=\boldsymbol{a}_{N_{4}}^{\mathrm{T}} \hat{\boldsymbol{x}}-e_{N_{q}}$

SE não cxistc tal índice, isto $\dot{e}, \boldsymbol{d}_{N} \leq \boldsymbol{a}_{N^{\mathrm{T}}}^{\mathbf{T}} \hat{\boldsymbol{x}} \leq \boldsymbol{e}_{N}$, ENTãO pare

(a solução atual é ótima obtida na iteração IT.)

\section{PaSSO 2: DIREÇÃO DUAL SIMPLEX}

Determine as componentes básicas da direção dual simplex: $\boldsymbol{\eta}_{B}=-\left(B^{\mathrm{T}}\right)^{-1} \boldsymbol{a}_{N_{q}}^{\mathrm{T}}$.

\section{Passo 3: TAManho do passo}

Passo 3.1: PONTOS DE NÃO-DIFERENCIAÇÃO

Encontre $\delta_{i}$ por:

Caso a): $\delta_{i}=\left\{-\frac{\hat{\lambda}_{B_{i}}}{\eta_{B_{i}}}\right.$, lal que $\left.-\frac{\hat{\lambda}_{B_{t}}}{\eta_{B_{i}}} \geq 0, i=1, \ldots, n\right\}$

ou

Caso b): $\delta_{i}=\left\{\frac{\hat{\lambda}_{B_{1}}}{\eta_{B_{1}}}\right.$, tal que $\left.\frac{\hat{\lambda}_{B_{i}}}{\eta_{B_{1}}} \geq 0, i=1, \ldots, n\right\}$

(suponha que são $r$ valores de $\delta_{l}$ ).

SE $r=0$ ENTãO pare

(ou seja, não existe nenhum delta, o dual não tem solução ótima finita

e, portanto, o primal é infactível.)

\section{Passo 3.2: BUSCA UNIDIMENSIONAL}

Passo 3.2.1: Ordene o vetor de $\delta_{i}$ de forma a obter: $\delta_{p_{0}} \leq \delta_{p_{1}} \leq \ldots \leq \delta_{p_{r}}$.

Passo 3.2.2: Determine $\ell \leq r$ tal que: $\mathrm{h}_{i}>0$ e $\mathrm{h}_{r+1} \leq 0$,

em que $h_{0}>0$ é dado no passo 1 .

$h_{f+1}=h_{f}-\left(e_{R_{p i}}-d_{B_{r \prime}}\right)\left|\eta_{k^{\prime \prime}}\right|$

faça $\delta=\delta_{p_{1}}$. 
Passo 3.2.3: SE $h_{r+1}>0$ ENTãO pare $(h(\hat{\lambda}+\delta \eta) \rightarrow \infty$, isto é, o problema dual não tem solução ótima. logo o primal é infactível.)

\section{Passo 4: ATUALIZAÇÃo}

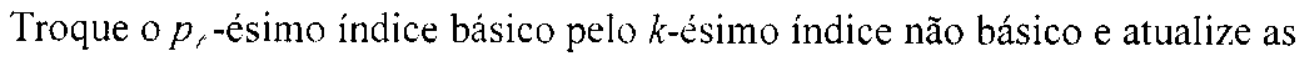
soluções primal e dual $B_{p_{i}} \leftrightarrow N_{g,}$.

$I T \leftarrow I T+1$, repita o passo 1 .

O passo 1 do algoritmo, caso a otimalidade não seja encontrada, determina a variável que entra na base $\left(\lambda_{N_{q}}\right)$ e o passo 3.2 , determina a variável que sai da base $\left(\lambda_{B_{p_{4}}}\right)$, caso o problema primal não seja infactível. Quando se fixa $t=1$, o método não faz a busca unidimensional e, assim temos o método dual simplex padrão ou usual, que iremos denotar por SB, isto é, sem busca.

A este procedimento de busca unidimensional exata, definido no passo 3.2, denominamos Busca Completa com ordenação preliminar ( $\mathrm{BC}_{-} 1$ ). Na seção a seguir, serão apresentadas algumas extensões para a busca unidimensional.

\subsection{Algumas Extensões da Busca Unidimensional}

Os métodos de busca para resolução de problemas de otimização, por exemplo,

Maximizar $\Phi(x)$

$x \in \Omega$,

têm dois passos fundamentais: a partir de uma solução $\hat{\boldsymbol{x}} \in \Omega$ i) determinação da direção de busca em $\hat{\boldsymbol{x}}: \boldsymbol{d}$ (normalmente direção de subida $\mathrm{em} \hat{\boldsymbol{x}}$ ) c, ii) determinação do tamanho do passo: $\hat{\delta}$ tal que $\Phi(\hat{\boldsymbol{x}}+\hat{\delta} \boldsymbol{d})>\Phi(\hat{\boldsymbol{x}})$. Para a determinação do tamanho do passo, muitas vezes é definido o subproblema:

Maximizar $\varphi(\delta)=\Phi(\hat{x}+\delta d), \delta \geq 0$. 
Entretanto, o esforço computacional para a resolução deste sub-problema pode ser grande e desnecessário, pois a direção de busca $\boldsymbol{d}$ é importante na solução $\hat{\boldsymbol{x}}$ e pode ser equivocada longe de $\hat{\boldsymbol{x}}$, sugerindo que uma nova direção de busca seja determinada. Embora o sub-problema para a função dual linear por partes seja de fácil resolução (passo 3.2 do algoritmo dual simplex com busca unidimensional exata), resta ainda saber se uma busca inexata (i.e., o abandono da direção de busca antes que sub-problema seja resolvido) pode ser efetiva. Investigamos três procedimentos de busca. O primeiro é busca inexata, que é baseado na estrutura linear por partes da função objetivo, somente uma parcela dos pontos de não-diferenciação são examinados, o segundo é um procedimento de busca exata, com uma modificação em BC 1 (Busca Completa versão dada no algoritmo), na busca inexata, adaptamos a clássica regra de Armijo, a qual apresenta um teste para evitar passos 'muito grandes".

\subsubsection{Primeira Modificação no Procedimento de Busca - Busca Parcial (BP)}

Este procedimento é uma alteração simples do passo 3.2.1 do algoritmo, por permitir a busca apenas entre uma parte dos pontos de não-diferenciação, ou seja, a busca no passo 3.2 .2 é feita entre os $\alpha r$ menores valores de $\delta$ s com $\alpha \cong 0,3$.

O passo 3.2.3 é também ligeiramente modificado pois não indica necessariamente que o primal seja infactível, isto é, dual ilimitado.

A motivação para a busca parcial decorreu da experimentação numérica que indicava $\ell \cong 0,3 r$ nas iterações iniciais para um grande número de problemas resolvidos (isto é, o número de deltas utilizados cra $\mathrm{cm}$ torno de $30 \%$ ).

\subsubsection{Segunda Modificação no Procedimento de Busca - Busca Completa (BC_ 2$)$}

Este procedimento é uma pequena modificação no passo 3.2 do algoritmo, que pode ser descrito pelo seguinte sub-algoritmo:

passo 3.2.1: Determine $p_{0}$ tal que: $\delta_{p_{1}}=\min \left\{\delta_{1}, i=1, \ldots, r\right\}$

Seja $h_{0}=d_{N_{q}}-\hat{y}_{N_{4}}$ ou $\hat{y}_{N_{4}}-e_{N_{4}}$ conforme o caso a) ou b),

$\ell=0$, e $E=\left\{p_{\theta}\right\}$. 
Passo 3.2.2: Determine $p_{i+1}$ tal que: $\delta_{p_{i+1}}=\min \left\{\delta_{,}, i=1, \ldots, r, i \notin E\right\} \mathrm{e}$

$$
\begin{aligned}
& h_{\ell+1}=h_{f}-\left(e_{B_{p_{i}}}-d_{B_{p_{i}}}\right)\left|\eta_{B_{p^{\prime}}}\right| \\
& E=E+\left\{p_{\ell+1}\right\}
\end{aligned}
$$

Passo 3.2.3: Se $h_{+1} \leq 0$ pare.

Senão

Se $\ell \leq r-1$ cntão $\ell-\ell+1$ e repita o passo 3.2.1;

Senão $\left(h_{r}>0\right)$ então pare

Note que o critério de parada no passo $2\left(h_{r}>0\right)$, indica que o problema dual não tem solução ótima e, portanto, o primal é infactível).

Este procedimento faz a busca exata sem que seja necessário a ordenação completa do vetor de pontos de não diferenciação, o que pode ser vantajoso caso $\ell$ seja muito menor do que $r$ como indica os experimentos numéricos (capítulo 7).

\subsubsection{Um Método de Busca Unidimensional Inexata - Regra de Armijo (AR)}

Descreveremos brevemente aqui, a regra de Armijo (Luenberger, 1984) especializada à função objetivo dual linear por partes.

Em essência, a regra de Armijo evita passos muito grandes.

Para que o tamanho do passo não seja muito grande, devemos ter (Figura 3.6):

$$
h(\hat{\lambda}+\delta \eta) \geq h(\hat{\lambda})+\delta\left(\varepsilon h_{0}\right)
$$

em que $\varepsilon \in(0,1)$. Então, enquanto:

$$
h\left(\hat{\lambda}+\delta_{p_{1}} \eta\right) \geq h(\hat{\lambda})+\delta_{p_{1}}\left(\varepsilon h_{0}\right)
$$

a busca continua.

Inicialmente começamos $\operatorname{com} \delta=\delta_{p_{0}}$, depois $\delta=\delta_{p 1}$ e assim sucessivamente até que: 
$h_{r, 1} \leq 0$ (i.c.. a busca exata foi concluída), ou

$h\left(\hat{\hat{\lambda}}+\delta_{p_{i-1}} \eta\right) \leq h(\hat{\hat{\lambda}})+\delta_{m_{i-1}}\left(\varepsilon h_{0}\right)$. (i.e., o passo $\delta_{p_{t+1}}$ é grande). $\Lambda$ dota-se $\delta=\delta_{p_{i}}$. Note que o menor passo para a regra de Armijo, vai ser sempre $\delta=\delta_{p_{11}}$. A Figura 3.6 ilustra a situação.

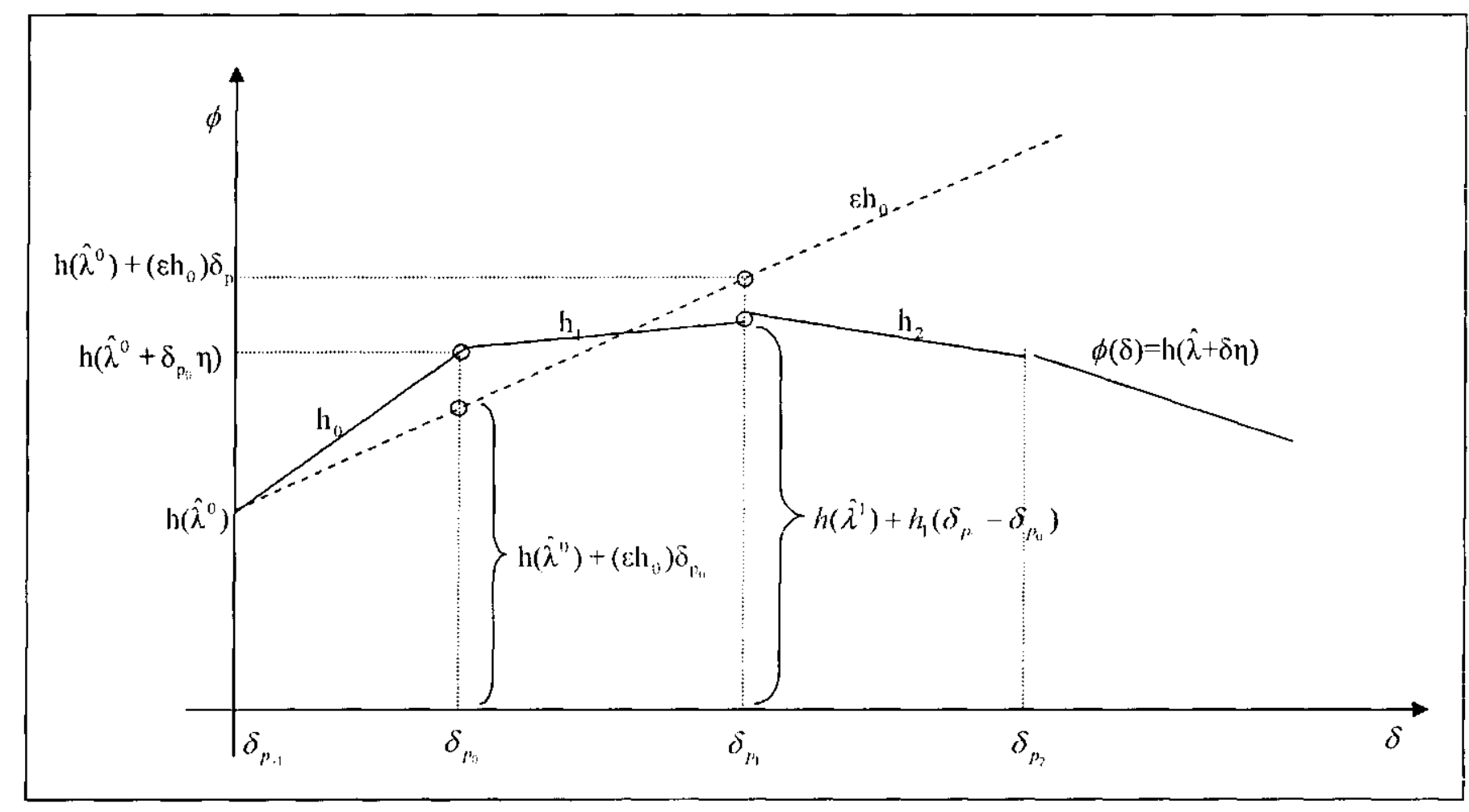

Figura 3.6 - Regra de Armijo para a função dual linear por partes

Para estes diferentes tipos de busca unidimensional descritos neste capítulo. realizamos extensivos testes computacionais e comparamos com o método dual simplex usual $t=p_{0}$ (Lemke, 1954). Os resultados são dados no capitulo 7. 


\section{Capítulo 4 - Técnicas de Esparsidade para o Método Simplex}

Neste capítulo são apresentadas algumas das técnicas mais utilizadas na implementação do método simplex para resolver problemas de otimização linear de grande porte quando se faz uso da decomposição LU na matriz base. Assim, uma atenção especial é dada às técnicas de esparsidade tais como as heurísticas de decomposição (fatoração) Grau Mínimo (Vanderbei, 1997) e Markowitz (1957) e os métodos de atualização da base de Forrest e Tomlin (1972) e de Reid (1982). Antes da apresentação desses métodos, descreveremos brevemente o trabalho clássico de Bartels e Goloub (1969), já que ele é a base para o desenvolvimento dos métodos citados acima.

$\mathrm{Na}$ última seção, apresentamos uma ferramenta que não encontramos na literatura no contexto do método simplex, conhecida como método iterativo do gradiente bi-conjugado (Lanczos, 1952), que foi utilizado na resolução dos sistemas básicos do método dual simplex com busca unidimensional descrito no capítulo 3.

\subsection{Introdução}

Sabemos que a fatoração $L U$ necessita da ordem de $m^{3}$ operações e as substituições progressiva e regressiva são algoritmos de ordem $m^{2}$ para matrizes densas. SegundoVanderbei (1997), tipicamente a fatoração leva de 10 a 100 vezes mais tempo do que substituições progressiva e regressiva. Portanto, é importante realizar o menos possível a fatoração $\boldsymbol{L U}$. Como no método 
simplex os sistemas básicos diferem por apenas uma coluna de uma iteração para a outra, então pode-se utilizar a matriz básica atual para o cálculo de uma nova basc. Scjam $\boldsymbol{B}$ a base atual (para a qual a fatoração já foi realizada) e $\overline{\boldsymbol{B}}$ a base na nova itcração. Então $\overline{\boldsymbol{B}}$ é simplesmente a matriz $\boldsymbol{B}$ com a coluna $\boldsymbol{a}_{i}$ (associada à variável que sai da base) substituída pela coluna $\boldsymbol{a}_{j}$ (associada à variável que entra na base) e isto pode ser algebricamente descrito da seguinte forma:

$$
\bar{B}=\boldsymbol{B}+\left(\boldsymbol{a}_{j}-\boldsymbol{a}_{i}\right) \boldsymbol{e}_{i}^{T},
$$

em que $\boldsymbol{e}_{i}$ é a $i$-ésima coluna da matriz identidade.

Como $\boldsymbol{B}$ é inversível, cntão a equação (4.1) pode ser escrita como:

$$
\bar{B}=\boldsymbol{B}\left(I+\boldsymbol{B}^{-1}\left(\boldsymbol{a}_{j}-\boldsymbol{a}_{i}\right) \boldsymbol{e}_{i}^{T}\right)
$$

Seja $\boldsymbol{E}$ a matriz entre parênteses. Denotando $\boldsymbol{d}_{B}=\boldsymbol{B}^{-1} \boldsymbol{a}_{j}$ e observando que $\boldsymbol{B}^{-1} \boldsymbol{a}_{i}=\boldsymbol{e}_{l}$, a matriz $\boldsymbol{E}$ pode ser reescrita da seguinte forma:

$$
E=I-\left(d_{B}-e_{i}\right) e_{i}^{T}
$$

Note que a matriz $\boldsymbol{E}$ na equação (4.3) é uma matriz esparsa e fácil de ser obtida. Se $\boldsymbol{E}$ tem inversa, então pode-se usa-lá com a decomposição $\boldsymbol{L} \boldsymbol{U}$ de $\boldsymbol{B}$, para fornecer uma maneira eficiente de resolver os sistemas envolvendo a nova base $\overline{\boldsymbol{B}}$. A não singularidade de $\boldsymbol{E}$ decorre do fato que $\boldsymbol{d}_{B i} \neq$ 0 , na escolha do passo na direção simplex.

Assim a idćia de escrever a próxima base como produto da base atual por uma matriz facilmente inversivel, pode ser estendida para várias iterações. Por exemplo, para $t$ iterações do método simplex, pode-se escrever:

$$
B^{t}=B^{\prime \prime} E^{\prime)} E^{l} \ldots E^{\prime-1}
$$

em que $\boldsymbol{B}^{t}$ é matriz básica na iteração $t$.

Ao evitar a fatoração até a $t$-ésima iteração, temos uma economia de tempo computacional, já que a decomposição foi realizada apenas uma vez para a base inicial $\boldsymbol{B}^{0}$. Por outro lado, os erros na aritmética do ponto flutuante tendem a crescer com as iterações tornando-se significativos e a quantidade de memória necessária para representar as novas matrizes $E^{j}$ na equação (4.4) também 
cresce, além dos erros de arredondamento. Assim, se fàz necessária uma refatoração periódica da base. Segundo Vanderbei (1997), na prática o período entre as fatorações pode ser um parâmetro definido pelo usuário, e um valor usual seria em torno de 100 iterações do método simplex. Na seção 4.9 abordamos o assunto mais detalhadamente. Neste capítulo, estudamos como devem ser feita a decomposição das matrizes básicas esparsas e suas atualizações. Na próxima seção damos um pequeno histórico de alguns trabalhos relevantes que existem na literatura, para $\mathrm{cm}$ seguida descrevê-los.

\subsection{Histórico}

Em 1969, Bartels e Golub propuseram uma maneira de atualizar a base, que apresenta bons resultados em relações aos erro de arredondamento, porém não considera a esparsidade. No trabalho de Forrest e Tomlin (1972) foi feita a decomposição da base utilizando a heurística de Markowitz (1957) e a atualização levando-se em conta a esparsidade, sem se preocupar com os erros de arredondamento. Mais tarde Reid (1982) propôs uma variante do método de Bartels e Golub, que preserva a esparsidade e reduz a propagação dos erros. Reid também utilizou a heurística de Markowitz (1957) para a decomposição. O livro do Vanderbei (1997) reúne esses trabalhos de uma forma resumida, propondo ainda uma heurística de decomposição chamada de Grau Mínimo, que será descrita posteriormente.

\subsection{Sistemas Lineares Esparsos}

A resolução de sistemas lineares esparsos do tipo $\boldsymbol{B} \boldsymbol{x}=\boldsymbol{b}$ de ordem $n \times n$, é de grande interesse em diversas áreas da ciência. Sendo assim, é natural que vários métodos tenham sido desenvolvidos a fim de facilitar a realização dessa tarefa nas últimas décadas. Um sistema linear é dito esparso quando a matriz $\boldsymbol{B}$ contém um pequeno número de elementos não-nulos.

Existem basicamente dois tipos de métodos de resolução de sistemas lineares: direto $\mathrm{e}$ iterativo. Entre os métodos dirctos, destacam-se o método de eliminaçâo de Gauss e variantes. Para os métodos iterativos destacamos o método do Gradiente Conjugado c Bi-Conjugado (Lanczos 1952, método de Gaus-Seidel e etc.

Os métodos diretos que realizam operações sobre a matriz do sistema alterando seus elementos, podem perder eficiência à medida que cresce o número de equações e o sistema torna-se mais esparso (ordem de milhares). Geralmente. esses métodos produzem muitos preenchimentos 
(surgimento de novos elementos não-nulos) o que não ocorre com os métodos iterativos e a complexidade $\left(O\left(n^{2}\right)\right)$ é muito alta para sistemas esparsos de grande porte.

Por outro lado, os métodos iterativos dependem de condições de convergência, mas quando aplicáveis tendem a ser mais eficientes para resolver sistemas de grande porte.

Os métodos diretos não são indicados para a resolução de sistemas lineares esparsos de grande porte, apesar da decomposição $L U$ conseguir bons resultados para o método simplex (Suhl e Suhl, 1990). (Duff et al, 1986) e (Silva. 2002). Mas enquanto os métodos diretos têm convergência garantida, os métodos iterativos dependem de critérios de convergência.

\subsection{Heurística de Markowitz}

Quando se trabalha com matrizcs esparsas, a necessidade de fazer permutações de linhas e/ou colunas é alta devido à possibilidade de se ter pivôs nulos e também de manter a esparsidade nas matrizes $L$ e $U$. Porém, encontrar a melhor permutação é um problema NP-difícil (Suhl e Suhl, 1990). Assim, justifica-se o uso de heurísticas simples que ajudam a preservar a esparsidade nas matrizes triangulares. Um exemplo pequeno e simples de uma permutação (ordenação) que evita o aparecimento de novos elementos diferentes de zero na decomposição é dado na Figura 4.1.
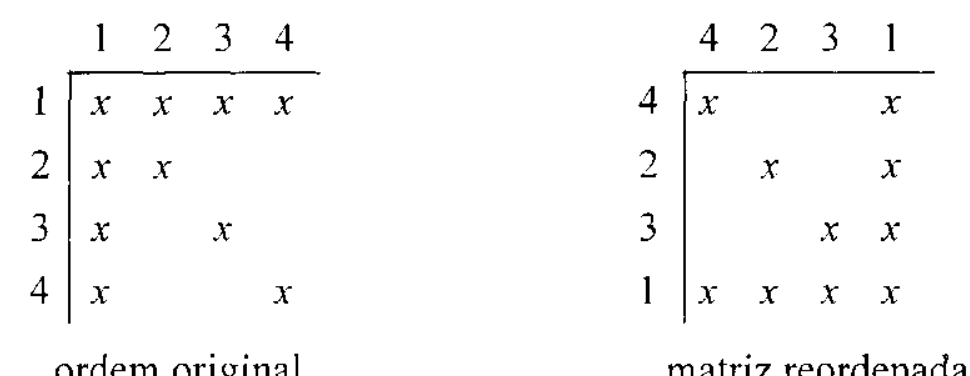

Figura 4.1-Permutação que preserva a esparsidade na decomposição $\boldsymbol{L U}$.

Uma heurística muito simples, mas que tem uma estratégia eficiente para manter a esparsidade na decomposição, é a Heurística de Markowitz (1957). A cada passo da eliminação seleciona-se como pivô um elemento da submatriz em que está sendo feita a decomposição, que representa o menor produto entre o número de elementos não nulos na sua linha e o número de elementos não nulos da coluna em que ele se encontra. Essa estratégia de ordenação ć uma das mais usadas na prática e apresenta resultados extremamente satisfatórios (Duff et al., 1986). 
Suponha que a eliminação Gaussiana se encontra no passo $k$, a matriz original que chamamos aqui de $\boldsymbol{B}$ (quadrada) tem dimensão $m$ e na submatriz ativa de ordem $(m-k), r_{i}^{(k)}$ é o númcro de elementos não nulos da linha $i$ nessa submatriz. e $c^{k}$, é o número de clementos não nulos da coluna $j$. A heurística de Markowitz seleciona como pivô o clemento $a_{i j}^{(k)}$ na submatriz que não seja tão pequeno numericamente e que minimize a expressão:

$$
\left(r_{i}^{(k)}-1\right)\left(c^{(k)}-1\right)
$$

Observe que, se existe uma linha ou coluna com um único elemento não nulo, esta heurística seleciona tal elemento como pivô, evitando assim o aparecimento de qualquer novo elemento diferente de zero.

A estratégia usual para controlar o erro de arredondamento na decomposição da matriz, é o pivoteamento parcial, que consiste em escolher como pivô o maior clemento em módulo da $k$-ésima coluna da submatriz ativa, ou seja, o novo pivô $b_{k k}^{(k)}$ será o elemento da $k$-ésima coluna que satisfaz a desigualdade abaixo:

$$
\left|b_{k k}^{(k)}\right| \geq\left|b_{t k}^{(k)}\right|, \quad i>k
$$

Se a linha que contém o novo pivô for a linha $p \neq k$, então deve-se fazer a troca da linha $p$ com a linha $k$, de modo que o elemento $b_{p k}^{(k)}$ fique na posição $(k, k)$ da submatriz.

Para garantir estabilidade numérica, é importante que o elemento pivô não seja muito pequeno em relação ao tamanho dos outros elementos da submatriz ativa. Sabemos que o pivoteamento parcial produz, em geral, uma certa estabilidade numérica, mas quando a esparsidade está presente, apenas cste método não é suficiente, pois ele não se preocupa com a geração de novos elementos não nulos, o que pode ser chamado de "preenchimento". Fntão, para que a estabilidade seja mantida na heurística de Markowitz, o candidato a pivô deve também satisfazer a desigualdade:

$$
\left|b_{i k}^{(k)}\right| \geq u \max _{p}\left|b_{p k}^{(k)}\right|, k=1, \ldots, m
$$

em que $u$ é um parâmetro no intervalo $0<u \leq 1$. 
Este procedimento para obter cstabilidade pode não ser muito útil se os elementos de $\boldsymbol{B}$ são de magnitudes extremamente diferentes (Suhl c Suhl, 1990). Quando isso ocorre, faz-se uma mudança de escala nos elementos da matriz.

\subsection{Heurística de Grau Mínimo}

Uma outra heurística bastante simples que pode scr utilizada para a decomposição de uma matriz $\mathrm{cm} \boldsymbol{L U}$, preservando a esparsidade é a Heurística de Grau Mínimo (Vanderbei, 1997), que pode ser descrita pelos seguintes passos:

1- Selecione a linha mais esparsa como linha pivô na submatriz ativa, digamos linha $i$ :

2- Sclecione um clemento não nulo nesta linha, cuja coluna na submatriz tenha o menor número de elementos diferente de zero, digamos coluna $j$;

3- O elemento $(i, j)$ é o pivô.

O número de elementos diferente de zero na submatriz ativa de uma linha ou coluna é chamado Grau da linha ou da coluna.

Não se pode afirmar qual destas heurísticas (Markowitz e Grau Mínimo) é a melhor, porém experimentos computacionais indicam que a de Markowitz é superior, (Duff et. al., 1986, Suhl e Suhl, 1990). Tal heurística vem sendo utilizada em alguns pacotes de programação linear (Suhl e Suhl, 1990), como por exemplo, o software CPLEX. No capitulo 7, apresentamos os resultados computacionais obtidos com cada uma destas heurísticas para uma classe de problemas esparsos.

\subsection{Método de Atualização de Bartels e Golub}

O esquema de decomposição de Bartels e Golub (1969) usado para a atualização da matriz básica tem propriedades numéricas interessantes, porém apresenta dificuldades práticas na implementação para problemas de grande porte e esparsos.

Inicialmente, faz-se a decomposição da matriz básica recorrendo à técnica de pivoteamento parcial, para evitar a propagação descontrolada dos erros de arredondamento.

Suponha que a matriz $B$ (permutada) seja decomposta $\mathrm{cm} L U$, em que $U$ é uma matriz triangular superior e $L$ é uma matriz triangular inferior, em que os elementos da diagonal desta última são iguais a 1. Observe que a decomposição $\boldsymbol{B}=\boldsymbol{L} U$ é equivalente a eliminação de Gauss no 
sentido que multiplicar $\boldsymbol{B}$ a esquerda por $\boldsymbol{L}^{-1}$ tem o efeito de fazer operações com as linhas de $\boldsymbol{B}$ para obter a matriz triangular superior $\boldsymbol{U}$.

Considere que a base inicial seja $\boldsymbol{B}, r$ seja a coluna básica que deixa a base $\mathrm{e} k$ a coluna de $N$ que entra na base, na primeira iteração. A nova matriz básica difere da anterior apenas por uma coluna. Assim o produto $\boldsymbol{L}^{-1} \boldsymbol{B}^{(1)}=\bar{U}$ difere da matriz $U$ também por uma única coluna. A matriz $\bar{U}$ é ilustrada pela Figura 4.2 .

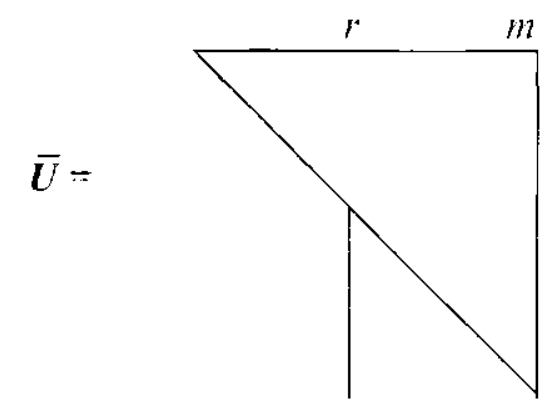

Figura 4.2 - Matriz triangular superior com uma coluna espeto, em destaque.

Definição 4.1 - Coluna (linha) espeto é a coluna (linha) que contém pelo menos um elemento diferente de zero abaixo da diagonal principal.

Bartels e Golub (1969) propuseram usar a seguinte permutação de colunas na matriz $\bar{U}$ acima: mova a coluna espeto para a última posição na matriz, e mova todas as colunas que estão a direita da coluna espeto uma posição para a esquerda, para obter a matriz denotada por $\boldsymbol{H}$, que chamamos de sub-triangular superior, (veja a Figura 4.3).

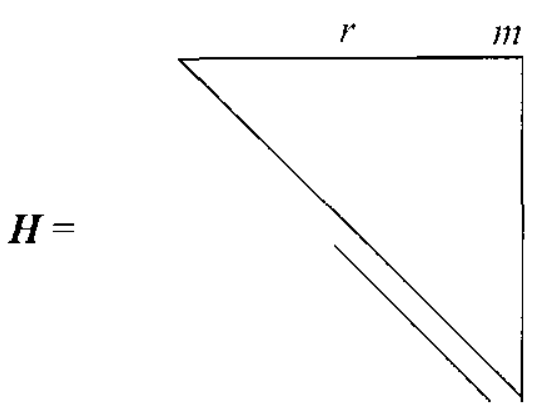

Figura 4.3- A matriz $\bar{U}$ reordenada 
Para reduzir $\boldsymbol{H}$ a uma matriz triangular superior pode-se usar operações elementares para eliminar os clementos da subdiagonal da coluna $r$ até a coluna $m$. A seleção do pivô é realizada em cada passo da eliminação, podendo haver ou não troca entre duas linhas adjacentes. Assim, a matriz triangular superior é obtida aplicando uma sequiència de operações elementares por linha em $\boldsymbol{H}$ (isto é equivalente a multiplicar $\boldsymbol{H}$ a esquerda por matrizes elementares). Este procedimento garante que os multiplicadores serão em módulo menores ou iguais a 1, mas ele tem a desvantagem que potencialmente todos os elementos nas linhas e colunas de $r$ a $m$ da matriz $\boldsymbol{H}$ são mudados e, assim, a matriz resultante triangular superior pode conter muitos novos elementos diferentes de zero. Isto reduz a eficiência do método para problemas esparsos de grande porte, já que esses novos elementos serão armazenados, utilizando a operação de inserção numa lista (tipo de estrutura de dados usada na implementação), a qual não é trivial e requer mais memória. Forrest e Tomlin (1972) propuseram uma maneira diferente de obter a matriz triangular superior, a partir da matriz $\boldsymbol{H}$, que se encontra na próxima seção.

\subsection{Método de Atualização de Forrest e Tomlin (1972)}

Os autores desenvolveram um método para a atualização da inversa da base, que transforma a matriz $\boldsymbol{H}$ (Bartels e Golub, 1969) numa matriz triangular superior preservando a esparsidade.

Inicialmente, a matriz básica $B$ ć decomposta em $L U$ utilizando a heurística de Markowitz. (1957), para reduzir o aparecimento de novos elementos diferente de zero na decomposição.

$\Lambda$ estratégia adotada consistc $\mathrm{cm}$ primciro eliminar os elementos da linha $r$, nas colunas de $r$ a $m$-1 da matriz de $\boldsymbol{H}$, pela multiplicação a esquerda de $\boldsymbol{H}$ por uma matriz $\boldsymbol{S}$ e, em seguida, realizar permutação de linhas para torná-la uma matriz triangular superior. Seja $\boldsymbol{S}=\boldsymbol{I}-\boldsymbol{e}_{r} \boldsymbol{S}^{\mathrm{T}}$, em que $\boldsymbol{I}$ é a matriz identidade, $\mathbf{e}_{\mathrm{r}}$ é o vetor de zeros exceto pela $r$-ésima posição que é 1 , e $\boldsymbol{s}^{\mathrm{T}}=\overline{\boldsymbol{u}} U^{1}$, para $\overline{\boldsymbol{u}}=\left(0, \ldots, 0, u_{r} u_{r+1}, \ldots, u_{r+m}\right)$. Matricialmente, temos:

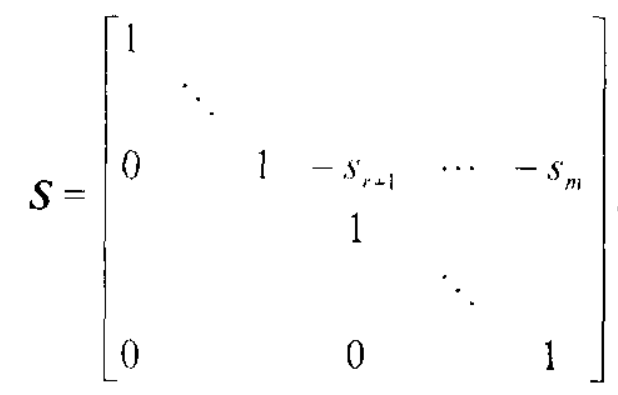

Observe que: 


$$
S U=\left(I-e_{r} S^{\mathrm{T}}\right) U=U-e_{r} \bar{u} U^{1} U=U-e_{r} \bar{u}
$$

Esta última expressão é simplesmente a matriz $U$ com os elementos nas posições $(r, r+1), \ldots,(r, m)$ nulos.

Agora, seja $\boldsymbol{V}=\boldsymbol{S} \boldsymbol{H}$, então $\boldsymbol{V}$ difere de $\boldsymbol{H}$ pelos elementos nas posições $(r, r), \ldots,(r, m-1)$ que foram substituídos por zero. Aplica-se nesse ponto uma matriz de permutação em $V$ para resultar numa matriz triangular superior.

A Figura 4.4 ilustra uma aplicação do procedimento descrito acima.

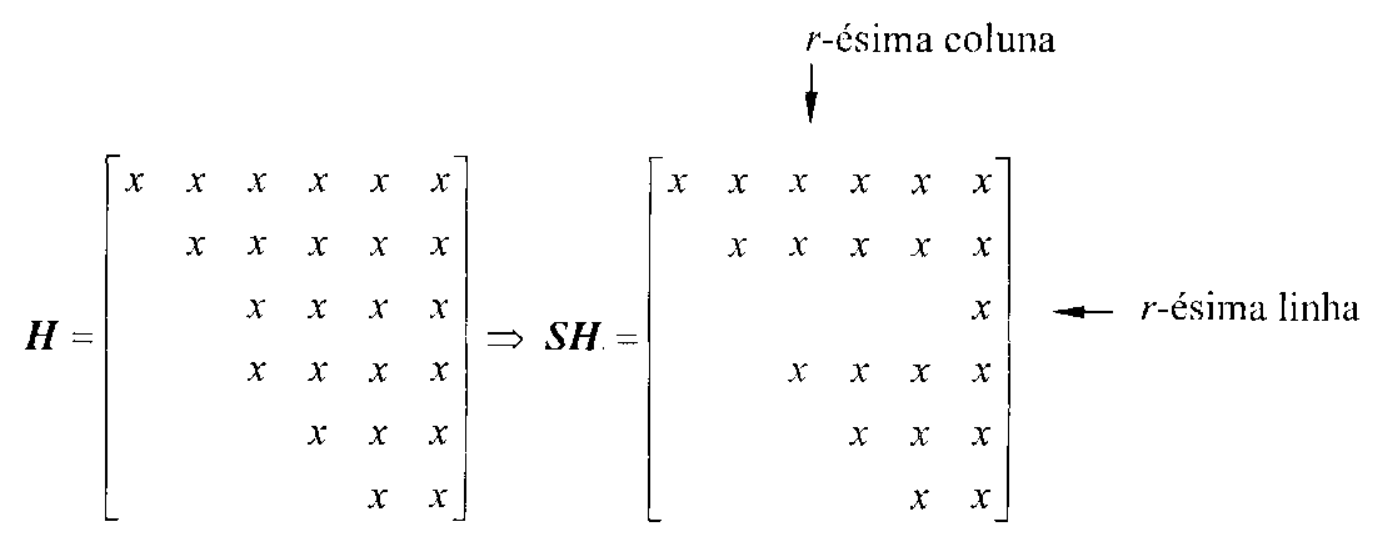

Figura 4.4 - Eliminação dos elementos da linha $r$.

Após esta operação, faz-se a uma permutação nas linhas da matriz $\boldsymbol{S H}$. Na Figura 4.4, scria colocar a $r$-ésima linha no lugar da $m$-ésima linha, e subir as linhas $r+1, r+2, \ldots . m$ uma posição acima, obtendo a seguinte matriz:

$$
\boldsymbol{P} \boldsymbol{V}=\left[\begin{array}{llllll}
x & x & x & x & x & x \\
& x & x & x & x & x \\
& & x & x & x & x \\
& & & x & x & x \\
& & & & x & x \\
& & & & & x
\end{array}\right]
$$

Observe que este procedimento faz com que a esparsidade seja preservada para a matriz triangular superior.

$\Lambda$ presentaremos a seguir um outro exemplo. em que na Figura 4.5 comparamos a diferença do método de Bartels e Golub (1969) e o de Forrest e Tomlin (1972). 


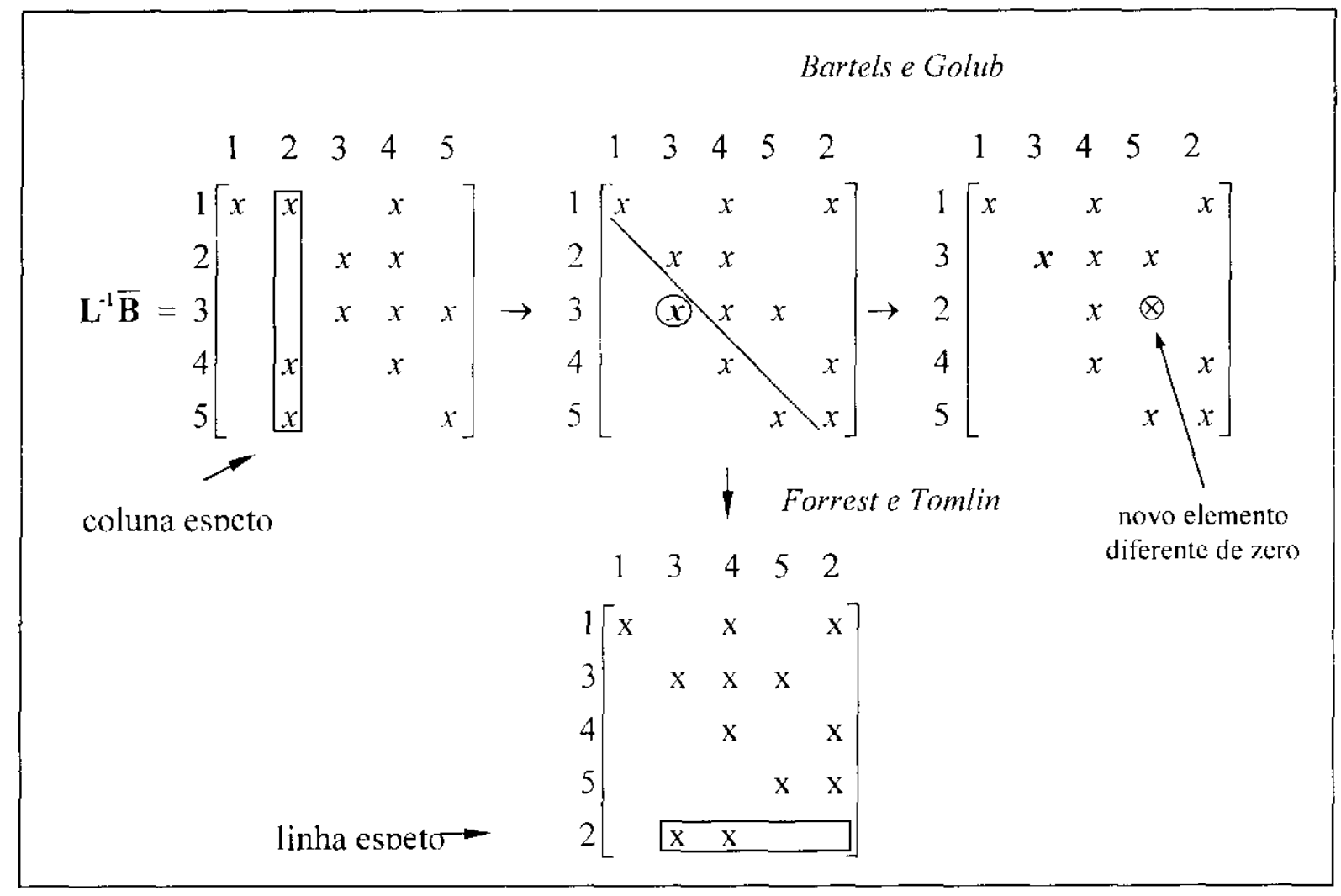

Figura 4.5-Comparação dos métodos: Bartels \& Golub $\times$ Forrest \& Tomlin.

Observe na Figura 4.5 que o primeiro passo no método de Forrest e Tomlin foi fazer a troca das linhas c depois será feita a eliminação dos elementos na linha espeto $(r=2)$, diferente do que foi descrito. Isto foi feito somente para esclarecer o método, sem alterá-lo $\mathrm{cm}$ essência.

Assim, o aparecimento de novos elementos diferente de zero fica confinado à linha espeto (veja a Figura 4.5), que muitas vezcs é csparsa. Reid (1982) melhorou este procedimento, que descrevemos na próxima seção.

\subsection{Método de Atualização de Reid}

Para descrevermos o método de atualização da base proposto por Reid (1982), considere uma matriz 9×9 ilustrada na Figura 4.6, para a qual o método de Forrest e Tomlin (1972) foi aplicada até 
o momento de colocá-la na forma de uma matriz com uma linha espeto, utilizando-se para isso, somente permutações.

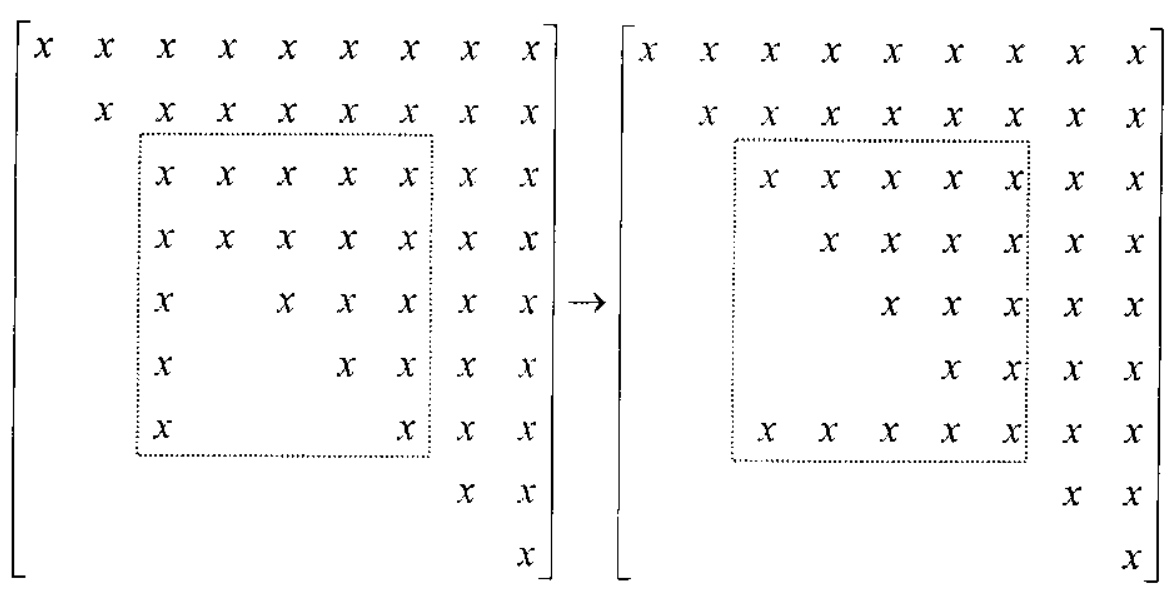

Fugura 4.6-A submatriz (pontilhada) no método de Forrest e Tomlin.

A submatriz não triangular em destaque na Figura 4.6, é criada da seguinte forma: como a linha espeto está na coluna 3 , as colunas vão desta posição até à coluna que corresponde a linha do último elemento não nulo (posição 7) da linha espeto. $\Lambda$ primeira linha da submatriz começa na posição que corresponde a coluna espeto e vai até o último elemento não nulo da coluna.

Seguindo o método de Forrest e Tomlin (1972), teríamos os elementos da última linha da submatriz zerados, através de operações elementares. Então Reid (1982) teve a idéia de reduzir a ordem desta submatriz até quando for possível, fazendo permutações de linhas/colunas e desse modo colocar a matriz original na forma triangular superior ou então na forma subtriangular superior, com menos elementos não nulos na subdiagonal, diminuindo portanto, o número de eliminações a serem realizadas.

Esta redução é feita da seguinte forma: determinada a coluna espeto, a submatriz não triangular $\mathrm{e}$ fazendo as permutações necessárias para obter a linha espeto, o elemento mais a esquerda da linha espeto é zero e a coluna que o contém é uma coluna que possui apenas um elemento não nulo e na diagonal. Então, essa coluna que possui apenas um elemento não nulo e na diagonal e a linha topo correspondente, não farão parte da submatriz não triangular.

A mesma idéia pode ser estendida para qualquer coluna da submatriz não triangular, que possui apenas um elemento não nulo e na diagonal, só que agora deve-se mover tal coluna para a posição mais a esquerda da submatriz ativa, colocando as colunas intermediárias uma posição para a direita, e aplicar a mesma permutação para as linhas, movendo as linhas intermediárias uma posição para baixo, de modo que o elemento não nulo desta coluna fique na posição $(1,1)$ da 
submatriz não iriangular. Depois de permutar as linhas e colunas, a submatriz não triangular pode ser reduzida no tamanho novamente. Esse processo deve ser realizado até que não se tenha mais colunas que possuam apenas um clemento nào nulo $\mathrm{e}$ na diagonal, na submatri\% não triangular.

$O$ procedimento acima se aplica para as linhas da submatriz não triangular que possucm apenas um clemento não nulo $\mathrm{e}$ na diagonal. Caso exista uma linha com tal caractcrística, devemos movê-la para a parte de baixo da submatriz. não triangular $\mathfrak{e}$ a coluna correspondente deve ser colocada na posição mais à direita da submatriz ativa. Depois dessas permutações, a coluna a direita e a linha na parte de baixo podem ser removidas da submatriz não triangular (a submatriz é redefinida, excluindo-se a última linha e última coluna).

Quando não houver mais colunas $\mathrm{c}$ nem linhas que possuam somente um elemento não nulo $\mathrm{c}$ na diagonal, permutação de colunas devem ser feitas com o objetivo de colocar a submatriz. não triangular na forma subtriangular superior. Essa tarefa consiste em colocar a coluna espeto no final da submatriz não triangular e mover as outras colunas da submatriz uma posição a esquerda.

Observe que como a dimensão da submatriz não triangular fica reduzida, as chances de encontrar mais colunas ou linhas que possuem somente um elemento não nulo e na diagonal aumentam.

Apresentaremos a seguir, um exemplo (Reid. 1982), onde o procedimento descrito por elc está ilustrado nas Figuras 4.7 a 4.13 .

Considere uma matriz (dimensão $9 \times 9$ ) que não está na forma triangular superior por causa da coluna espeto, para ser colocada na forma totalmente triangular superior.

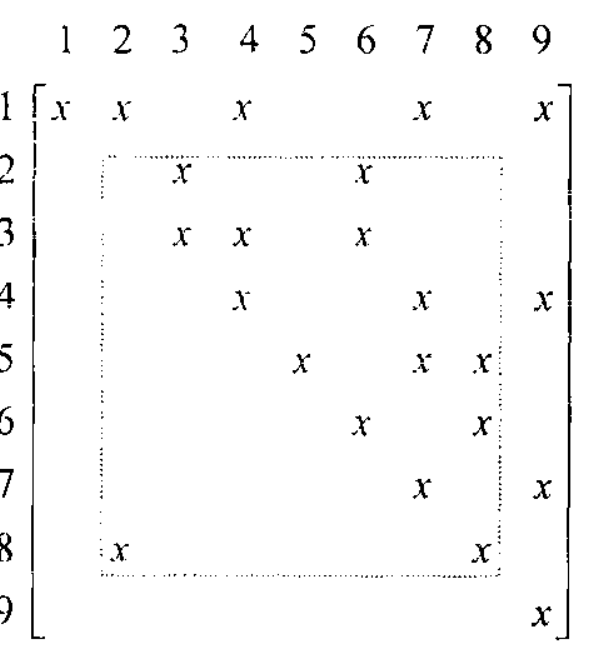

Figura 4.7 - Matriz original. 
Como vemos, a linha espeto é a linha 8 e o elemento mais a sua esquerda é zero. A coluna deste elemento é uma coluna que possui apenas um elemento não nulo e na diagonal, então a primeira coluna e a primeira linha não farão parte da submatriz não triangular. A coluna cspeto é a coluna $2 \mathrm{e}$ o seu último elemento não nulo está na linha 8 . Assim, a coluna $9 \mathrm{e}$ linha 9 também não farão parte da submatriz não triangular e, portanto, a matriz submatriz não triangular de dimensão $7 \times 7$ é composta pelas linhas e colunas de 2 a $8 . \Lambda$ linha espeto é realmente muito esparsa, sendo possivel aproveitar deste fato e reduzir a dimensão da submatriz não triangular, até não encontrarmos mais colunas ou linhas que possuam um único clemento não nulo e na diagonal.

Examinando a submatriz não triangular, vemos que a coluna 5 é uma coluna que possui um único elemento não nulo $\mathrm{c}$ na diagonal. Movendo essa coluna para a segunda coluna da matriz e a linha 5 para a segunda linha da matriz, temos:

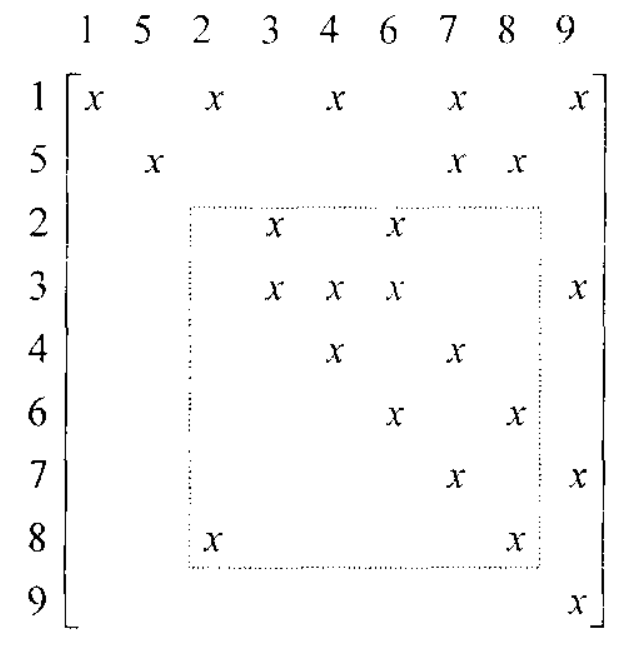

Figura 4.8 - Coluna com único elemento movida para frente da submatriz.

Então a linha 2 e coluna 2 serão removidas da submatriz não triangular e, com isso, a nova submatriz não triangular passa a ter dimensão $6 \times 6$.

Agora, notamos que a linha 7 é uma linha que possui um único elemento não nulo e na diagonal. Vamos, então, colocá-la na parte de baixo da submatriz não triangular, ou seja, na oitava linha da matriz e mover a coluna 7 para a oitava coluna da matriz, obtendo: 


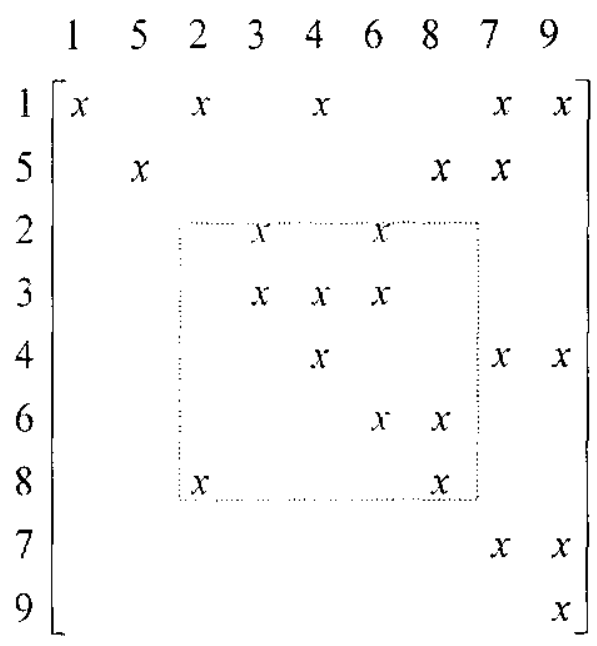

Figura 4.9 - Linha com único elemento movida para abaixo da submatriz.

Continuando, vemos que a linha 4 é uma linha que possui um único elemento não nulo e na diagonal. Vamos então colocá-la na sétima linha da matriz e mover a coluna 4 para a sétima coluna da matriz, obtendo:

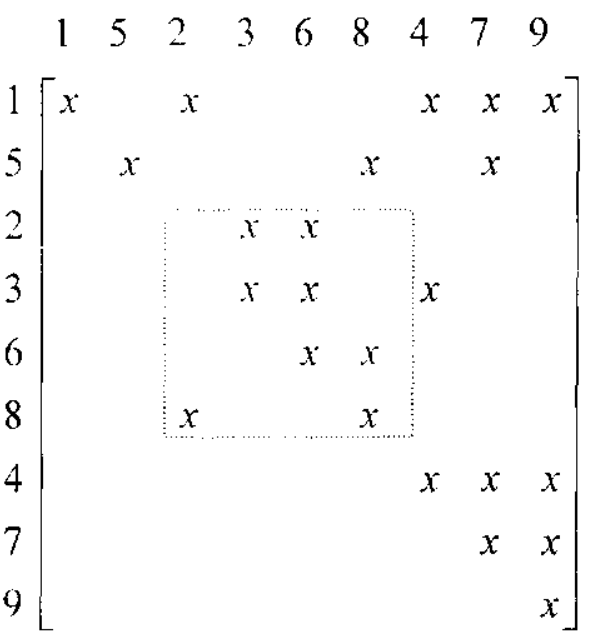

Figura 4.10 - Linha com único elemento movida para abaixo da submatriz.

Agora, colocaremos a matriz na forma subtriangular superior, movendo a coluna 2 para a sexta coluna da matriz, obtendo: 


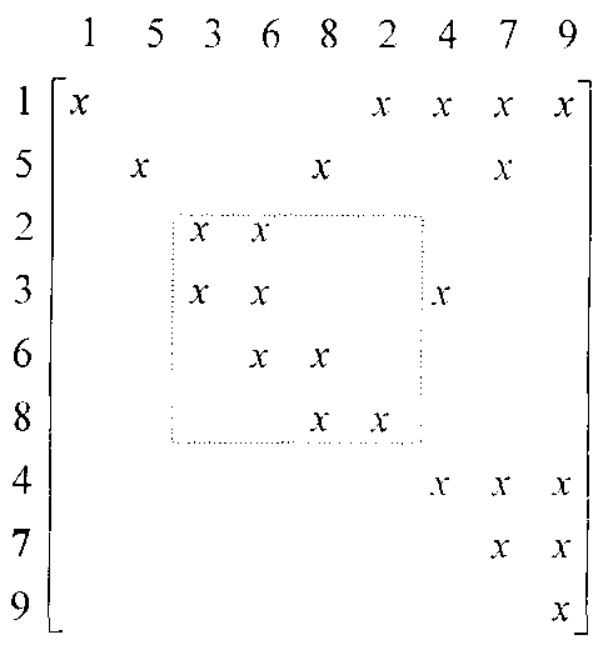

Figura 4.11 - Permutações que resultaram na Hessenberg superior.

Novamente, a coluna 2 é uma coluna que possui um único elemento não nulo e na diagonal. Movendo essa coluna para a terceira coluna da matriz e a linha 8 para a terceira linha da matriz, temos:

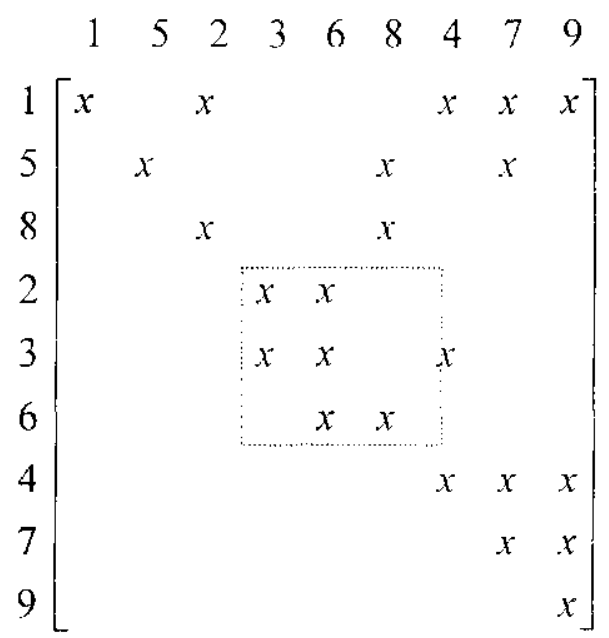

Figura 4.12 - Coluna com único elemento movida para frente da submatriz

Aqui, a coluna 8 é uma coluna que possui um único elemento não nulo e na diagonal. Permutamo-na com a quarta coluna da matriz e a linha 6 com a quarta linha da matriz e obtermos: 


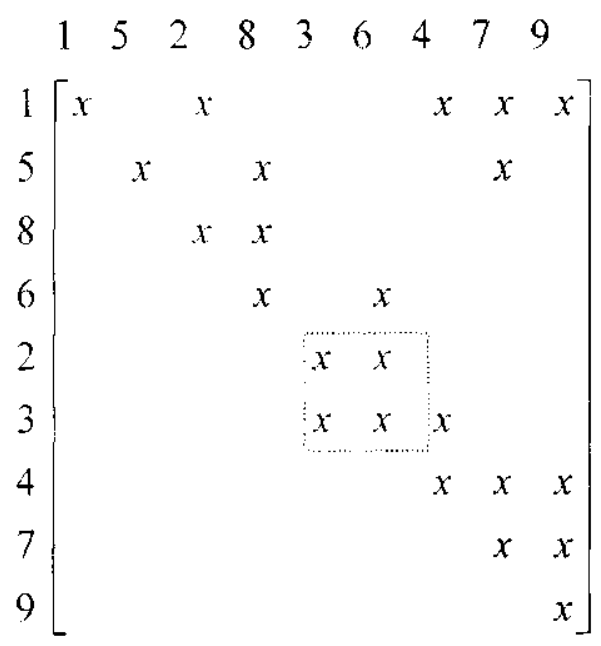

Figura 4.13 - Coluna com único elemento movida para frente da submatriz.

Finalmente, observamos que a submatriz nâo triangular não tem mais colunas ou linhas que possuam somente um elemento nâo nulo e na diagonal. Isso quer dizer que o processo terminou e a matriz resultante está mais próxima da forma triangular superior, faltando eliminar um número reduzido de elementos fora da diagonal.

\subsection{Refatoração da Matriz Básica}

Nas seções anteriores descrevemos duas heurísticas de decomposição e 3 métodos de atualização da matriz básica. O fato de decompor a matriz básica inicialmente e aproveitar esta decomposição para que possa ser atualizada durante todo o processo do método simplex até que a solução ótima seja encontrada, pode acarretar em acúmulo nos erros de arredondamento mesmo se empregar um critério de estabilidade numérica, como descrito na heurística de Markowitz. Por outro lado, decompor várias vezes a matriz básica tem um custo computacional alto. Assim, surge a seguinte questão: Quando c com que freqüência deve se fazer uma refatoração? Daremos a seguir alguns fatos que podem responder esta pergunta.

Podemos dizer que uma refatoração da matriz é necessária nos seguintes casos (Vanderbei, 1997):

- Quando a qualidade da solução está ameaçada. Tal ameaça, vem principalmente, com o aumento no tamanho dos elementos da matriz. Portanto, o tamanho do maior elemento deve ser monitorado, e uma refatoração deve ser feita se ele torna-se demasiadamente grande cm módulo. 
- Quando o número de opcrações elementares por linha $E_{\mathrm{i}}$ aumenta e o número de não nulos em $\boldsymbol{U}$ também aumenta no todo, vale a pena fazer uma refatoração completa da base atual.

Baseado na situação descrita anteriormente a questão agora é, com que freqüência uma refatoração da matriz deve ser feita. Scgundo Vanderbei (1997), podemos proceder da seguinte forma para se chegar em uma resposta:

Sejam

G - o número de operações aritméticas necessárias para fazer uma decomposição $L U$;

S - o número de operações para fazer a substituição progressiva e regressiva;

E - o número de operações para multiplicar uma matriz por um vetor.

Então, o número de operações para a iteração inicial do método simplex é:

$\mathrm{G}+2 \mathrm{~S}$

pois, é preciso fazer uma decomposição $L U$ e duas substituições progressiva/regressiva, uma para o sistema envolvendo a base e a outra para o sistema envolvendo a transposta da base.

Assim, na próxima iteração é necessário fazer duas substituições progressiva/regressiva e dois cálculos da inversa. Cada iteração subseqüente é igual a anterior, exceto que existem dois cálculos extras da inversa. Portanto, o número médio de operações aritméticas por iteração, se a refatoração for feita no passo $t$, é:

$$
\begin{aligned}
& N(t)=\frac{1}{t}((G+2 S)+2(S+E)+2(S+2 E)+\ldots+2(S+(t-1) E)) \\
& N(t)=\frac{1}{t} G+2 S+(t-1) E
\end{aligned}
$$

Relaxamos a integralidade de $t$, e determinamos o mínimo de $N(t)$ :

$$
\frac{\mathrm{dN}(\mathrm{t})}{\mathrm{dt}}=-\frac{1}{\mathrm{t}^{2}} G+E=0
$$

Portanto, 


$$
t=\sqrt{\frac{G}{E}}
$$

Assim, como E é de ordem $m$ e. se a matriz básica é densa, G é de ordem $m^{3}$, portanto t deve ser da ordem de $\sqrt{\frac{m^{3}}{m}}=\sqrt{m^{2}}=m$ iterações, ou seja, a refatoração deve ser feita depois de $m$ itcraçõcs, aproximadamente.

Agora, se G é uma matriz esparsa, G é da ordem de $m^{2}$ o que indica que a refatoração deve ser feita depois de $\sqrt{\frac{m^{2}}{m}}=\sqrt{m}$ iterações.

Na prática, o valor de $t$ é um parâmetro ajustável. Se não for informado usa-se $t=100$ (Vanderbei, 1997).

O pacote de otimização CPLEX não especílica o valor utilizado, segundo o manual é determinado automaticamente. Fm nossas implementações, utilizamos alguns valores para este parâmetro, os resultados estão relatados no capítulo 7 .

\subsection{Um Método Iterativo para o DSBU}

\subsubsection{Introdução}

Para resolver sistemas lineares do tipo $\boldsymbol{B} \boldsymbol{x}=\boldsymbol{b}$, em que a matriz $\boldsymbol{B}$ tem alguma propricdade, como por exemplo, simétrica definida positiva. os métodos iterativos convergem rapidamente (Saad, 2003).

Oishi (2004) utilizou o método do gradiente bi-conjugado (Lanczos, 1952) com sucesso nos problemas em dinâmica dos fluídos computacionais, em que a matriz dos coeficientes não era simétrica.

As matrizes básicas do método DSBU também não têm propriedades, como smetria ou definida positiva. Apesar disso, resolvemos investigar e implementar o método do gradiente biconjugado como ferramenta para resolver os sistemas básicos (lineares) a cada iteração do método duai simplex. 


\subsubsection{Método do Gradiente Bi-Conjugado (GBC)}

No método simplex grande parte do seu desempenho, em termos de tempo computacional, depende diretamente da resolução de três sistemas lineares que surgem a cada iteração.

Portanto, a escolha do método de resolução de sistemas lineares é um ponto importante para a resolução de cada problema. Um método de resolução eficiente armazena apenas os elementos não nulos utilizando estruturas de dados apropriadas, para que possa cconomizar operações aritméticas e memória.

Apresentamos agora, o método iterativo do gradiente bi-conjugado (GBC) para a solução de sistemas lineares esparsos do tipo $\boldsymbol{B} \boldsymbol{x}=\boldsymbol{b}$, com a matri dos coeficientes $\boldsymbol{B}$ não simétrica (Press et al. 1992).

O método constrói quatro sequêencias de vetores, $\boldsymbol{r}_{k}, \bar{r}_{k}, \boldsymbol{p}_{k} \mathrm{e} \overline{\boldsymbol{p}}_{k}, k=1,2, \ldots$. Deve-se fornecer os vetores iniciais $\boldsymbol{r}_{l}, \overline{\boldsymbol{r}}_{l}, \quad \boldsymbol{p}_{i}=\boldsymbol{r}_{i} \mathrm{e} \overline{\boldsymbol{p}}_{i}=\overline{\boldsymbol{r}}_{l}$. Desta forma, utiliza-se as seguintes fórmulas de recorrência:

$$
\begin{aligned}
& \alpha_{k}=\frac{\boldsymbol{r}_{k}^{\prime} \overrightarrow{\boldsymbol{r}}_{k}}{\overline{\boldsymbol{p}}_{k}^{\prime} \boldsymbol{B} \boldsymbol{p}_{k}} \\
& r_{k i}=r_{k}-c_{k} B p_{k} ; \\
& \vec{r}_{k j}=\bar{r}_{k}-\alpha_{k} B^{\prime} \bar{p}_{k} \\
& \beta_{k}=\frac{\overline{\boldsymbol{r}}_{k \cdot l}^{\prime} \boldsymbol{r}_{k \cdot j}}{\overline{\boldsymbol{r}}_{k}^{\prime} \boldsymbol{r}_{k}} ; \\
& \boldsymbol{p}_{k, 1}=\boldsymbol{r}_{k, 1}: \beta_{k} \boldsymbol{p}_{k} \\
& \bar{p}_{k j}=\bar{r}_{k},+\beta_{k} \bar{p}_{k}
\end{aligned}
$$

Esta seqüência de vetores satisfaz a condição de bi-ortogonalidade:

$$
\overline{\boldsymbol{r}}_{i}^{T} \boldsymbol{r}_{1}=\boldsymbol{r}_{i}^{T} \overline{\boldsymbol{r}}_{1}=0, j<i,
$$

e a condição:

$$
\bar{p}_{l}^{T} \boldsymbol{B} p_{i}=\boldsymbol{p}_{t}^{T} \overline{\boldsymbol{B}}^{T} \bar{p}_{,}=0, j<i
$$

Esses vetores são reciprocamente ortogonais: 


$$
\overrightarrow{\boldsymbol{r}}_{i}^{T} \boldsymbol{p}_{i}=\boldsymbol{r}_{1}^{T} \overline{\boldsymbol{p}}_{1}=0, j<i
$$

A prova destas propriedades é feita por indução (Fletcher, 1974). Se nenhum dos denominadores for zero, o algoritmo termina em $s$ passos com $s<n$ (Press et al, 1992) e $r_{m \cdot l}=\bar{r}_{m ! l}=0$.

Para utilizar o método, um valor inicial deve ser fornecido para o vetor $\boldsymbol{x}_{l}$. Escolha $\boldsymbol{r}_{\boldsymbol{l}}$ como o resíduo inicial,

$$
\boldsymbol{r}_{l}=\boldsymbol{b}-\boldsymbol{B} \boldsymbol{x}_{1}
$$

com $\vec{r}_{l}=\boldsymbol{r}_{l}$. O novo vetor da seqüência de aproximações é calculado por:

$$
\boldsymbol{x}_{k, 1}=\boldsymbol{x}_{k}+\alpha_{k} \boldsymbol{p}_{k}
$$

A equação (4.10) garante que $r_{k}$, das fórmulas de recorrência em (4.8) é de fato o resíduo $\boldsymbol{b}-\boldsymbol{B} \boldsymbol{x}_{k+1}$ correspondente a $\boldsymbol{x}_{k}$. Assim, se $\boldsymbol{r}_{\boldsymbol{m}, /}=0, \boldsymbol{x}_{k, 1}$ é a solução do sistema $\boldsymbol{B} \boldsymbol{x}=\boldsymbol{b}$.

Um critćrio de parada para o método do gradiente bi-conjugado é

$$
\frac{\left|\boldsymbol{b}-\boldsymbol{B} \boldsymbol{x}_{k+1}\right|}{|\boldsymbol{b}|}<t o l
$$

cm que tol é uma tolerância escolhida de acordo com o problema

Mesmo que não exista garantia de que este método convirja ou torne-se instável, para qualquer matriz $\boldsymbol{B}$, na prática isto é raro (Press ét al, 1992).

Como o método é mais eficiente se a matriz $\boldsymbol{B}$ for bem-condicionada (Quarteroni et al. 2000), pode-se utilizar um método de pré-condicionamento (Golub e Loan, 1989), isto é, o sistema linear original é transformado para:

$$
\left(\tilde{\boldsymbol{B}}^{-l} \boldsymbol{B}\right) \boldsymbol{x}=\tilde{\boldsymbol{B}}^{-l} \boldsymbol{b}
$$

em que $\tilde{\boldsymbol{B}}$ é chamada matriz pré-condicionadora (Quarteroni et al, 2000). A idéia é deixar a nova matriz dos coeficientes mais próxima da matriz identidade, fazendo com que a seqüência gerada 
convirja em poucas iterações. Este procedimento incorporado ao método GBC é conhecido como método do Gradiente Bi-conjugado Pré-condicionado (GBCP). A matriz $\tilde{\boldsymbol{B}}$ pode ser tomada como a diagonal de $\boldsymbol{B}$ (Oishi, 2004).

Para se utilizar esse procedimento, os vetores $z_{k} \mathrm{e} \bar{z}_{k}$, definidos como

$$
\tilde{\boldsymbol{B}} z_{k}=\boldsymbol{r}_{k} \text { e } \tilde{\boldsymbol{B}}^{T} \bar{z}_{k}=\overline{\boldsymbol{r}}_{k}
$$

são introduzidos nas fórmulas de recorrência (4.8). Note que as equações (4.12) representam sistemas lineares que devem ser resolvidos a cada iteração. Desta forma, a matriz $\tilde{\boldsymbol{B}}$ dever ser escolhida de forma que esses sistemas tenham soluções triviais.

Logo, a definição dos valores $\alpha_{k}$ e $\beta_{k}$ dos vetores $\boldsymbol{p}_{k}$ e $\overline{\boldsymbol{p}}_{k}$ serão modificados para

$$
\begin{gathered}
\alpha_{k}=\frac{\overline{\boldsymbol{r}}_{k}^{T} z_{k}}{\overline{\boldsymbol{p}}_{k}^{T} \boldsymbol{B} \boldsymbol{p}_{k}} ; \\
\beta_{k}=\frac{\overline{\boldsymbol{r}}_{k \cdot z_{k-1}^{T}}^{T}}{\overline{\boldsymbol{r}}_{k}^{T} z_{k}} ; \\
\boldsymbol{p}_{k, 1}=\boldsymbol{z}_{k, 1}+\beta_{k} \boldsymbol{p}_{k} ; \\
\overline{\boldsymbol{p}}_{k, 1}=\overline{\boldsymbol{z}}_{k, 1}+\beta_{k} \overline{\boldsymbol{p}}_{k} .
\end{gathered}
$$

O critério de parada para o $(\mathrm{GBCP})$ pode ser expresso como

$$
\frac{\left|\tilde{\boldsymbol{B}}^{-i}\left(\boldsymbol{b}-\boldsymbol{B} \boldsymbol{x}_{k+1}\right)\right|}{\left|\tilde{\boldsymbol{B}}^{-l} \boldsymbol{b}\right|}<t o l .
$$

Embora trabalhamos apenas com este método iterativo, existe outros métodos que podem ser testados e aplicados no método DSBU, como o Generalized Minimal Residual Algorithm - GMRES (Saad e Schultz, 1986 e Saad, 2003) que tem algumas propriedades teóricas interessantes e o Método do Gradiente Bi-Conjugado Estabilizado (Van der Vorst, 1992). 
Capitulo 5 - Ciclagem, Degeneração e Variantes do Método Simplex

Neste capítulo são apresentadas algumas técnicas utilizadas para impedir ciclagem, e outras para tratar com a degeneração e o fenômeno de estagnação. Além disso, citamos algumas variantes simplex com ênfase na regra de Dantzig Normalizada adaptada para o método dual simplex com busca unidimensional.

\subsection{Introdução}

Os métodos do tipo simplex percorrem soluções básicas, digamos, $\boldsymbol{B}^{0}, \boldsymbol{B}^{1}, \boldsymbol{B}^{2}, \ldots, \boldsymbol{B}^{t}, \ldots$ $\left(\boldsymbol{B}^{t}\right.$, base na iteração $\left.t\right)$, de modo que: $f\left(x^{\prime \prime}\right) \geq f\left(x^{1}\right) \geq f\left(x^{2}\right) \ldots \geq f\left(x^{\prime}\right) \geq \ldots \mathrm{cm}$ que $x^{\prime}$ é a solução básica associada à base $\boldsymbol{B}^{\prime}$ (no caso dual: $h\left(\pi^{0}\right) \leq h\left(\pi^{\prime}\right)<\ldots \mathrm{h}\left(\boldsymbol{\pi}^{\prime}\right)<\ldots \mathrm{cm}$ que $h$ é a função objetivo dual $\mathrm{e} \pi^{t}$ é a solução básica dual associada à base $\boldsymbol{B}^{\prime}$ ). Se as soluções básicas são não-degeneradas então: $f\left(x^{0}\right)>f\left(x^{1}\right) \ldots>f\left(x^{t}\right)>\ldots$ de modo que as soluções básicas são distintas e, portanto, nunca uma base é repetida. Entretanto, se soluções degeneradas ocorrem (as quais podem ser representadas por partiçôes básicas distintas), pode-se ter um seqüência de soluções básicas associadas as partições básicas distintas tal que:... $f\left(x^{\prime}\right)<f\left(x^{\prime-1}\right)=f\left(x^{l+2}\right)=\ldots=f\left(x^{\prime \prime k}\right)<f\left(x^{l+k+1}\right)$.

Dizemos que há uma "estagnação" do método (stalling) em $k$ iterações (um mesmo vértice é representado por distintas partições básicas. que ocorre somente se a solução for degenerada). Neste caso, embora ocorra estagnação, o método evolui c encontra um novo vértice na iteração $k+1$. 
Entretanto, poder-sc-ia ocorrer que $f\left(x^{i+1}\right)=\ldots=f\left(x^{l+k}\right)$ e as partições básicas associadas à $x^{t+1}$ e $\boldsymbol{x}^{t+k}$ serem as mesmas, de modo que o ciclo se repetiria infinitamente. Este fenômeno é chamado ciclagem

Embora degeneração abra a possibilidade de ocorrer ciclagem ela dificilmente ocorre na prática (Maros, 2003) . Este problema foi reconhecido e demonstrado por Beale (1955). Várias ferramentas foram desenvolvidas para evitar ciclagem, as duas mais conhecidas são o método simplex lexicográfico (Charnes. 1952) que é equivalente) à técnica de perturbação de Dantzig et al. (1955) segundo Tcrlaky (2000) e o método mais popular para evitar ciclagem é conhecido pela regra de Bland (1977) que foi discutida cm Sousa (2000).

Bland demonstrou que o método simplex é finito com esta regra. Entretanto, o desempenho computacional do método simplex, sob esta regra, decai ( $\Lambda$ vis e Chvátal, 1978).

Para a escolha da variável que entra na base a regra de Bland seleciona o menor índice entre as variáveis não básicas com custo reduzido negativo. Sc não existe tal variável a soluçâo atual é ótima. Para a escolha da variável que sai, o menor índice é escolhido tal que após a mudança, a base permaneça factível e o índice desta variável seja o menor entre todos aqueles com tal possibilidade.

\subsection{Ciclagem em Problemas de Otimização Linear}

O trabalho recente de Gass e Vinjamuri (2004) apresenta uma coleção de problemas que produzem ciclagem quando resolvidos manualmente pelo método simplex com os dados fracioinários e, por curiosidade, aplicamos o método dual simplex com busca unidimensional para estes problemas, cujos os resultados serão apresentados a seguir.

Segundo Gass e Vinjamuri, existem algumas condições necessárias para se construir um excmplo capaz de produzir o fenômeno de ciclagem no método simplex. Assim, considere um problema de otimização linear na forma padrão com $m$ restrições e $n$ variáveis, com empate na escolha da variável que entra ou sai da base (pela regra usual de Dantzig) e a escolha se faz pelo menor índice das candidatas. Com essa regra, para ciclagem ocorrer deve-se ter $m \geq 2, n \geq m+3$, e $n \geq 6$. Para ciclagem ocorrer em um vértice que não seja a solução ótima, deve-se ter $m \geq 3, n \geq$ $m+3$ e $n \geq 7$. Assim, um exemplo com somente duas variáveis não pode ciclar; um exemplo de ciclagem deve ter pelo menos 6 variáveis, o mínimo de duas restrições de igualdade e pelo menos três variáveis não básicas. Uma solução básica factível com degeneração simples (somente uma variável básica é zero) não pode ciclar (Gass, 1993) c. o 'comprimento' mínimo de um ciclo é de 6 iterações (Yudin e Golshtein, 1965). 
O primeiro exemplo de um problema em que o método simplex cicla foi construído por Hoffman (1953). Todos os exemplares a seguir estão no formato . I p (veja apêndice A).

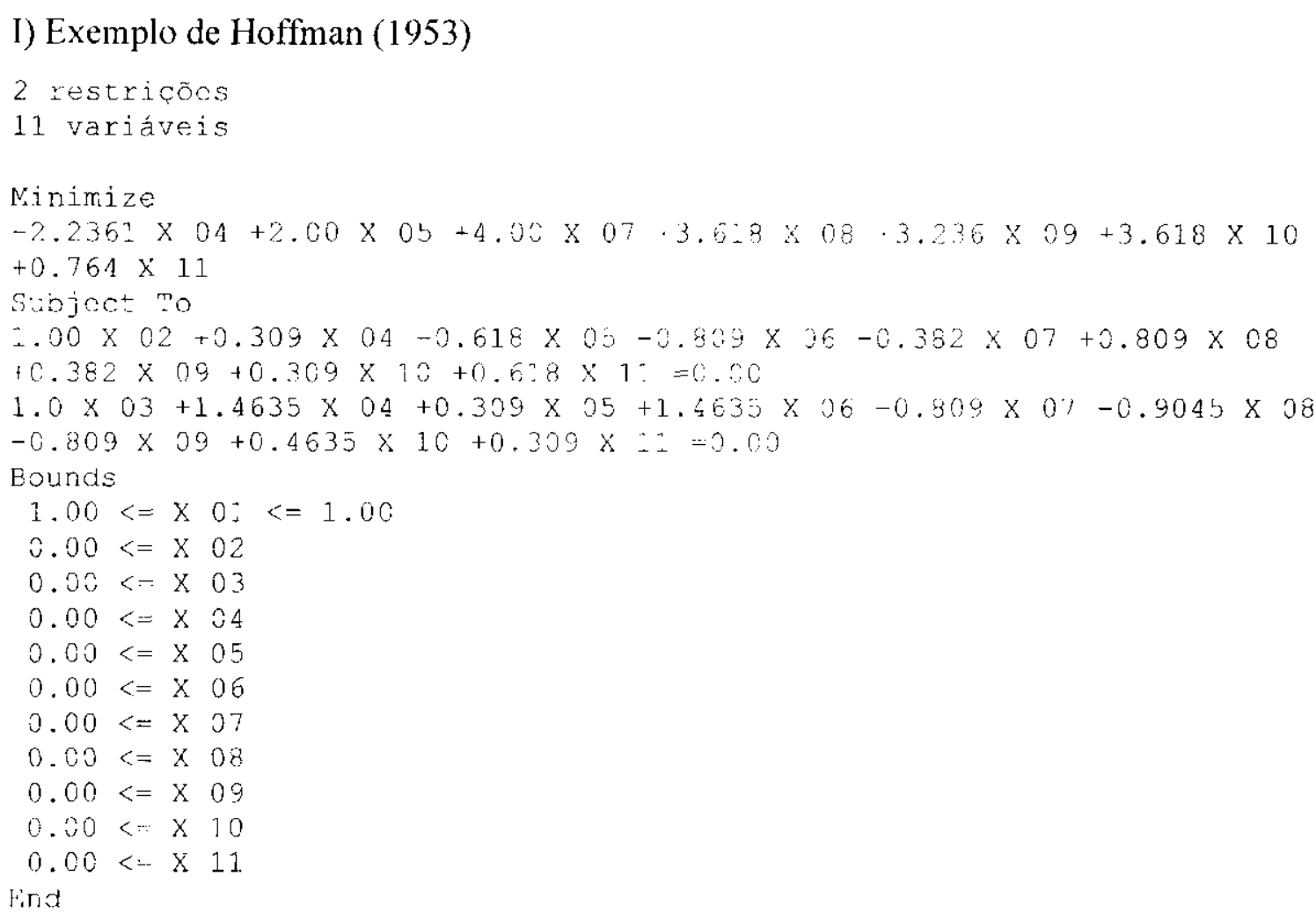

O método primal simplex cicla em 10 iterações sem reconhecer que a solução básica inicial $(\mathrm{X} 1=\mathrm{X} 2=\mathrm{X} 3-0)$ e todas as bases subseqüentes asseguram o mínimo à função objetivo (solução ótima).

Apresentamos agora, todos os problemas e as soluções, que foram relatados no trabalho de Gass e Vinjamuri, para $\mathrm{cm}$ seguida dar os resultados obtidos com o CPLEX e com o método dual simplex com busca unidimensional (Tabela 6.1).

II) Exemplo de Beale (Gass, 1985)

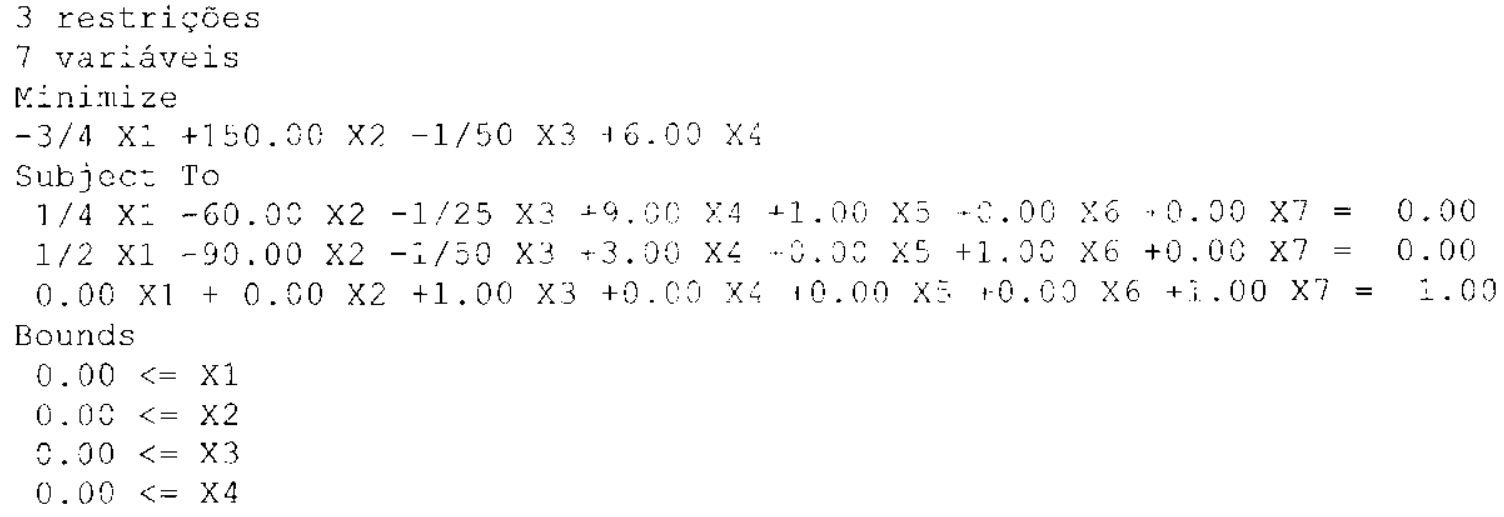




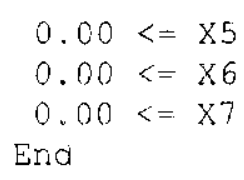

Solução: $x 1$ : $/ 25, \quad x 3=1, \quad x \leq=3 / 100$; função objetivo=-1/20; cicla 6 iterações

III) Exemplo de Yudin e Goldshtein (1965)

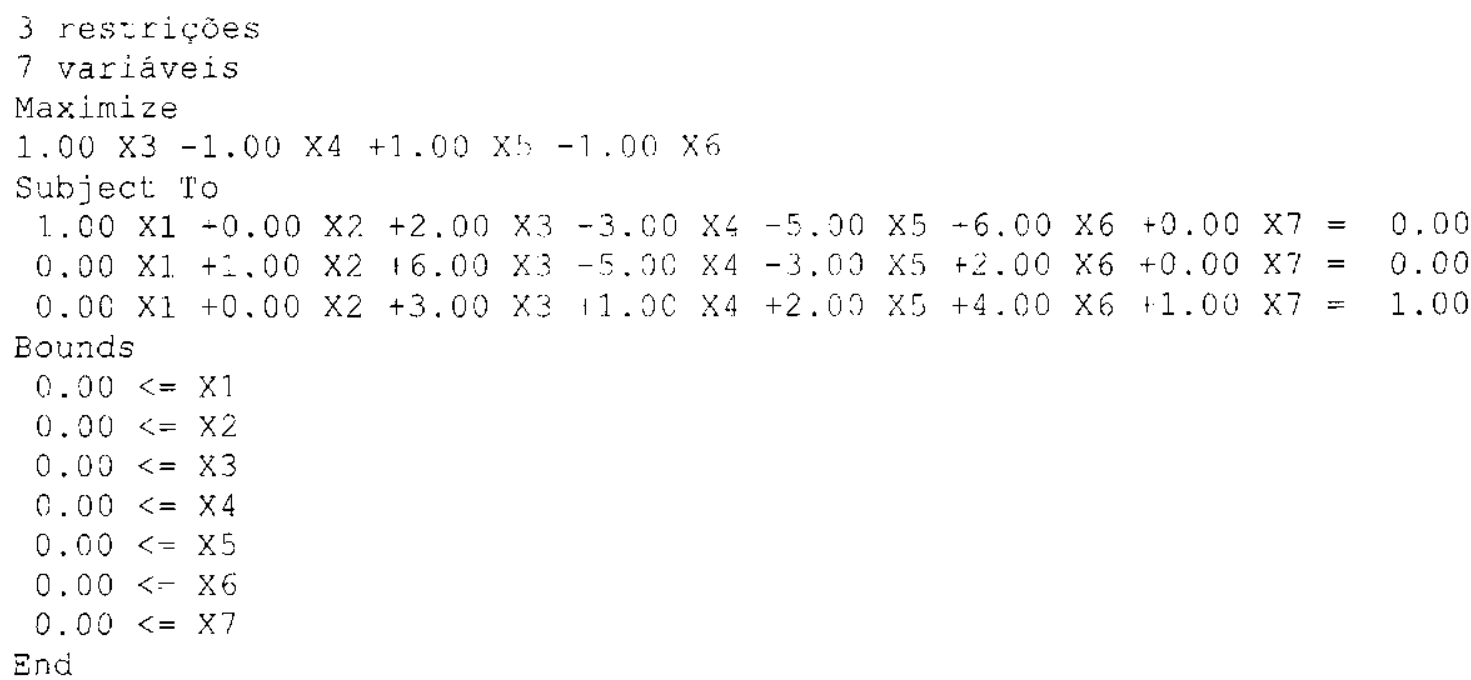

Solução: $x 1=2.5, \times 2 \cdots 1.5, \times 5 \quad 0.5$; função objetivo=0.5; cicla 6 iterações

IV) Exemplo de Yudin c Goldshtcin (1965)

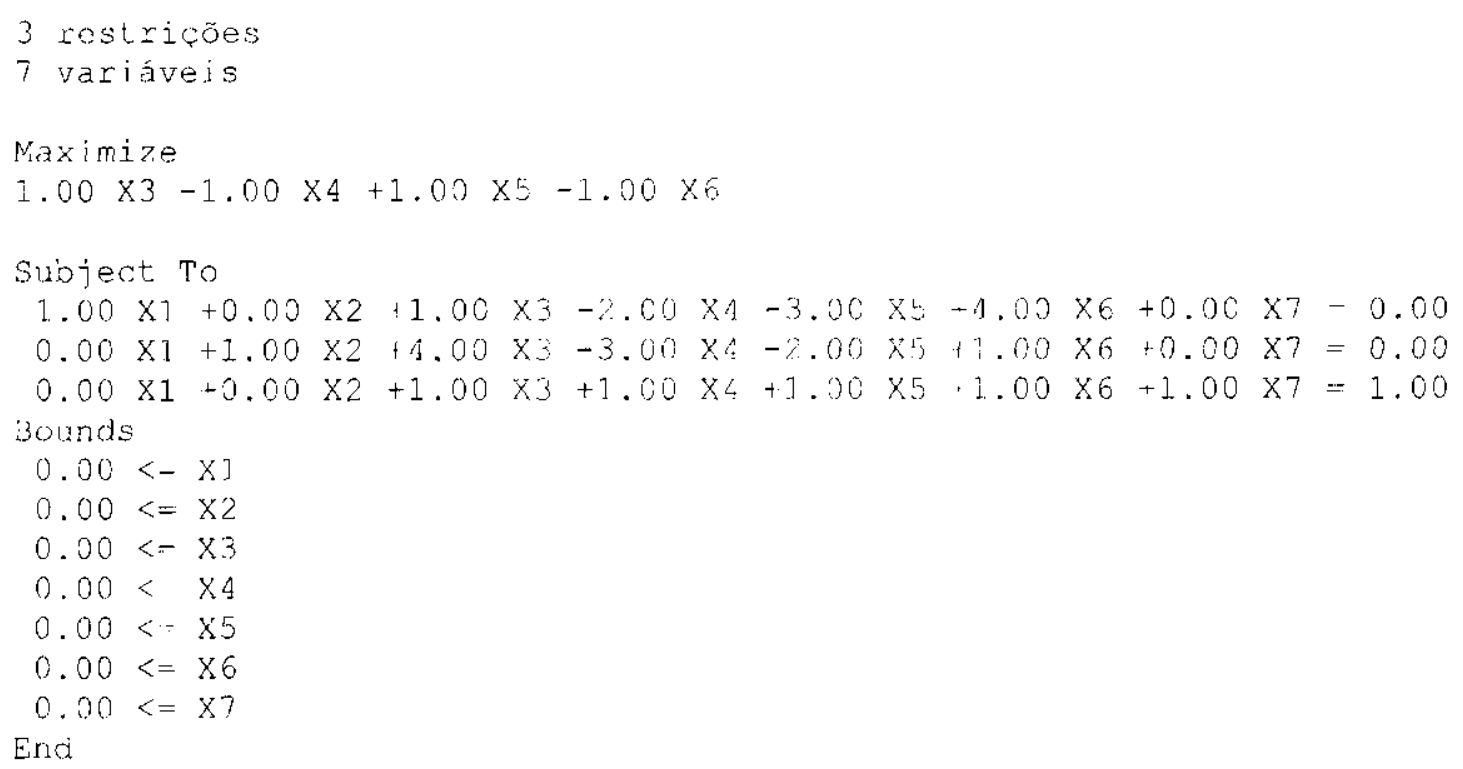

Solução: $\times 1=3, \quad \times 2:: 2, \quad x 5=1$; função objetivo- 1 ; cicla 6 iterações

V) Exemplo de Kuhn (Balinski e Tucker, 1997)

3 restriçós

7 variávels 


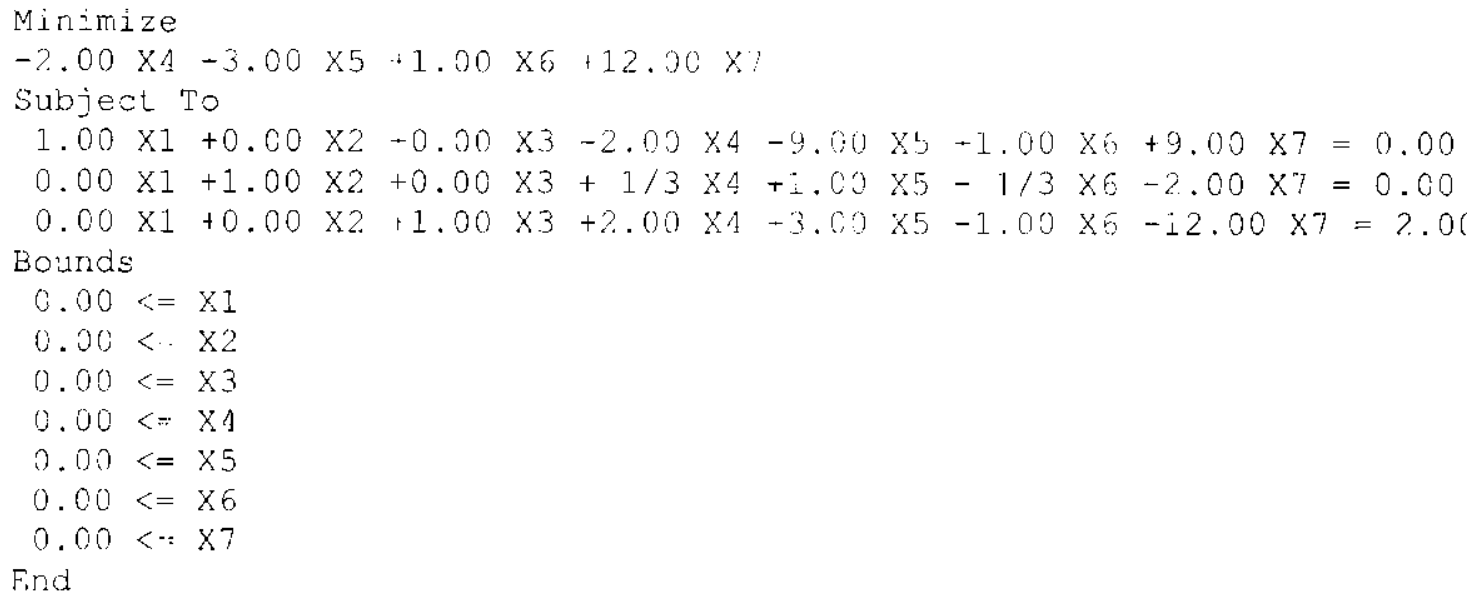

Solução: $x_{\perp}=2, \quad x_{4}=2, \quad x_{6}=2 ;$ função objetivo=-2: cicla 6 iterações

VI) Exemplo de Marshall e Suurballe (1969)

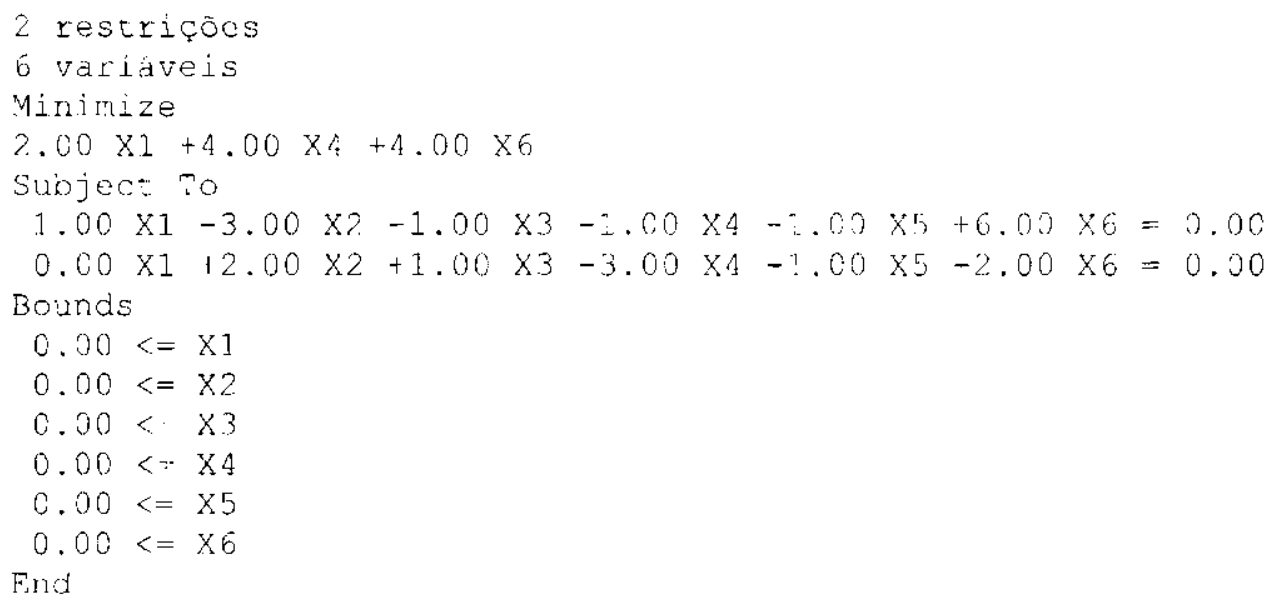

Solução: $x 1=x 2=x 3=x 4: x b=x 6 \cdot 0 ;$ Iunção objetivo $=()$; cicla 6 iterações

VII) Exemplo de Marshall e Suurballe (1969)

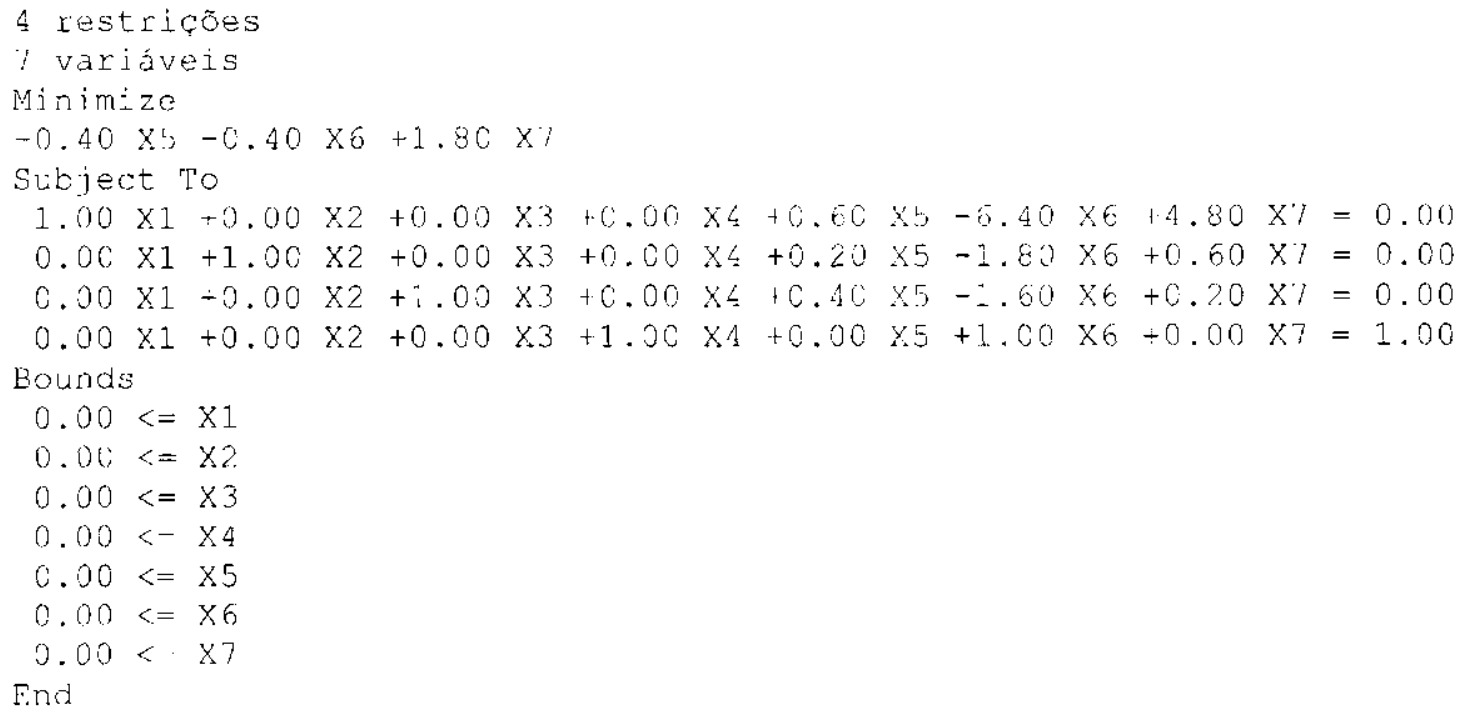


Solução: $x_{1}=4, \quad \times 2=1, \quad \times 5=4, \quad \times 6=1$; função objetivo=-2; cicla 6 iterações

VIII) Exemplo de Solow (1984)

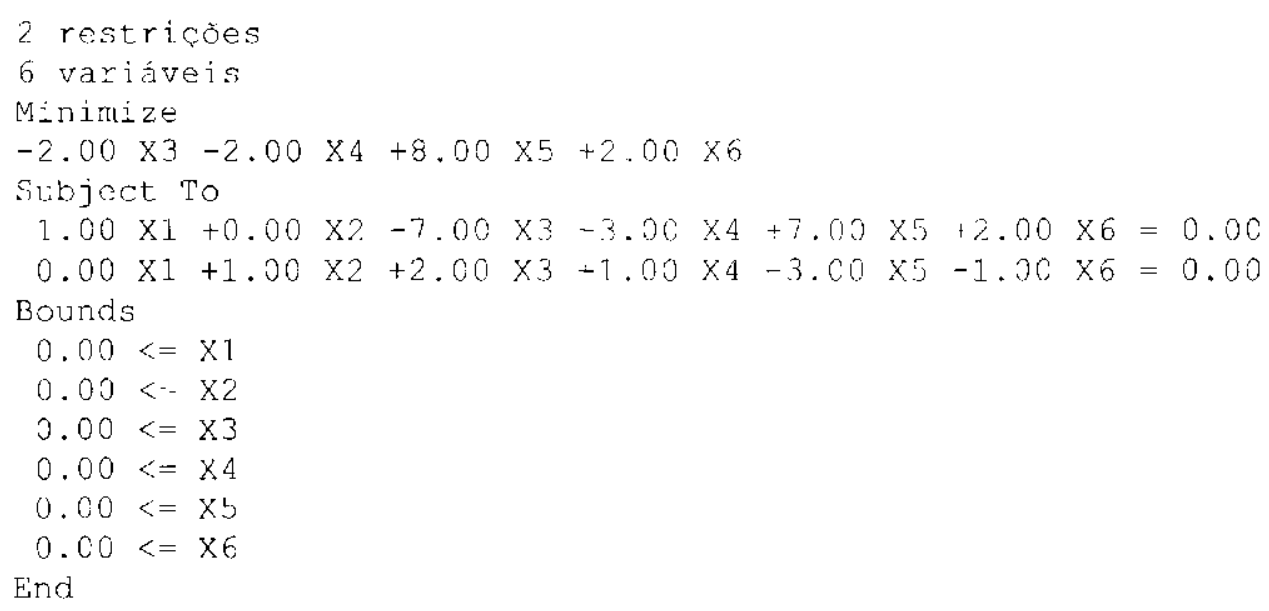

Solução: $\mathrm{x} 1=\mathrm{X} 2=\mathrm{X} 3=\mathrm{X} 4=\mathrm{X} 5=\mathrm{X} 6=\mathrm{c}$; função objetivo $=0$; Cicla 6 iterações

\section{IX) Excmplo de Sierksma (1996)}

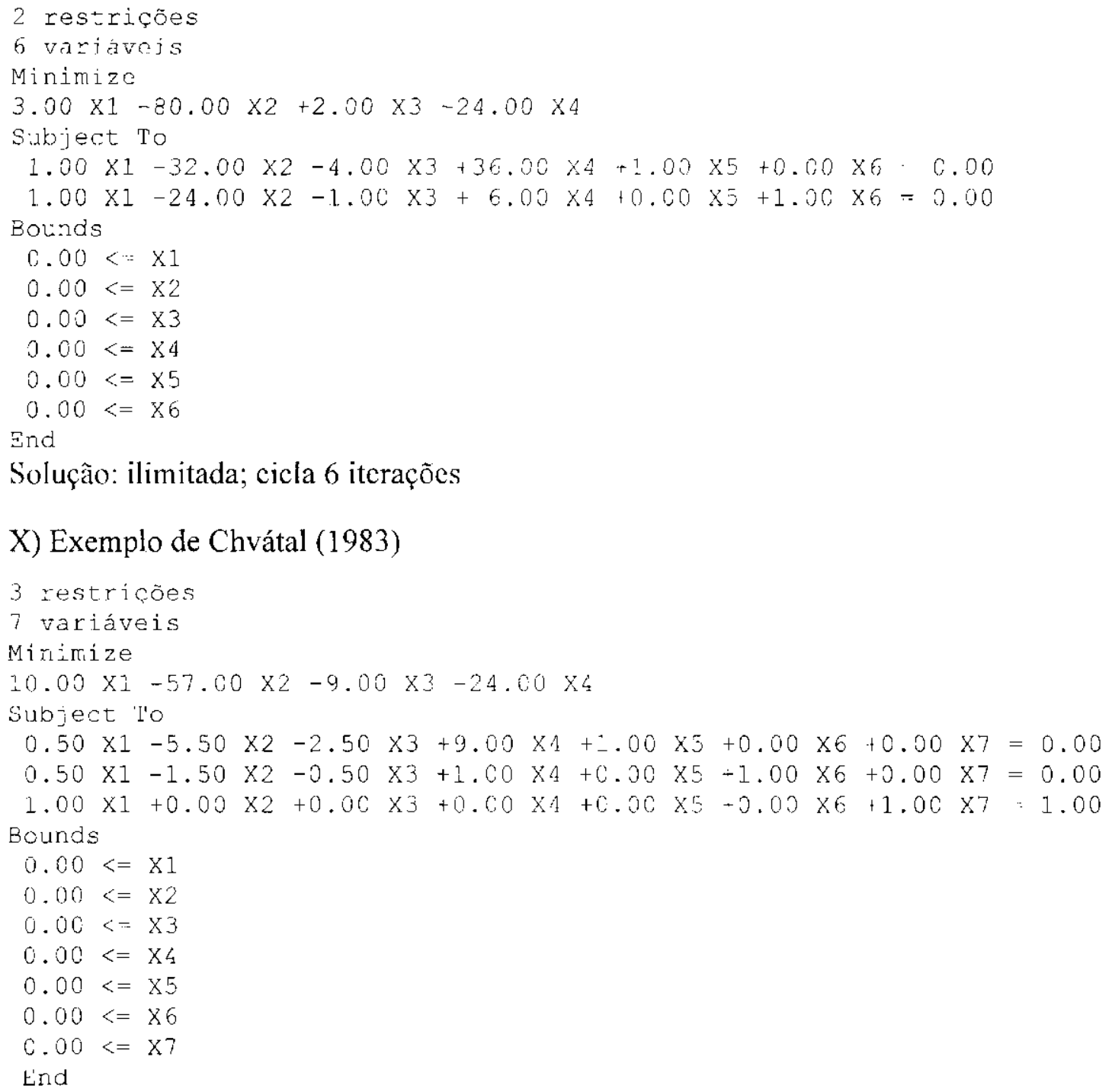


Solução: $\times 1=1, \quad x 3=1, \quad x 5=2$; função objetivo=1; cicla 6 iterações

XI) Excmplo de Nering e Tucker (1993)

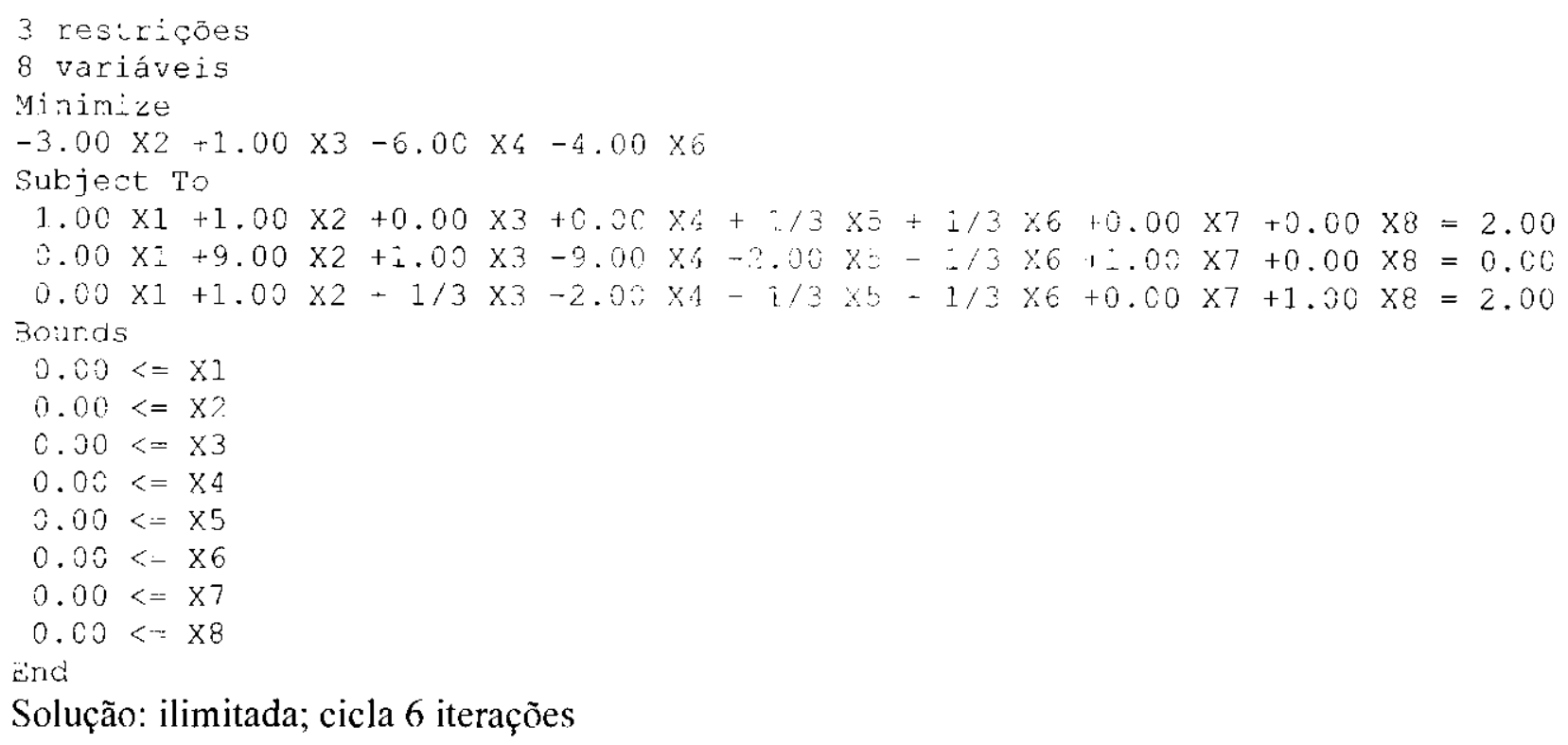

Solução: ilimitada; cicla 6 iterações

Observe que para alguns problemas há empate na escolha da variável que entra na base segundo a regra de Dantzig. Mas para haver ciclagem, depende da base inicial escolhida.

Na Tabela 5.1 são apresentados os resultados computacionais obtidos com o CPLEX e método dual simplex com busca unidimensional

\begin{tabular}{|c|c|c|c|c|}
\hline \multirow[b]{2}{*}{ prob/ema } & \multirow[b]{2}{*}{ nome } & \multicolumn{2}{|c|}{ iteraçōes } & \multirow[b]{2}{*}{$F O^{(4)}$} \\
\hline & & $C P L E X^{(2)}$ & $D S B U^{(3)}$ & \\
\hline 1 & Hoffman & 2 & 2 & 0.00 \\
\hline 2 & $B e a / e^{(t)}$ & 3 & 3 & -0.05 \\
\hline 3 & Yudin & 3 & 3 & 0.50 \\
\hline 4 & Yudin2 & 6 & 4 & -1.00 \\
\hline 5 & Kuhn & 4 & 5 & -2.00 \\
\hline 6 & Marshall & 0 & 0 & 0.00 \\
\hline 7 & Marshall2 & 4 & 4 & -2.00 \\
\hline 8 & Solow & 3 & 2 & 0.00 \\
\hline 9 & Sierksma & 2 & 2 & ilimitado \\
\hline 10 & Chvátal & 4 & 3 & 1.00 \\
\hline 11 & Nering $^{(1)}$ & 4 & 3 & ilimitado \\
\hline
\end{tabular}

\footnotetext{
${ }^{(1)}$ Problemas com os dados fracionários:

(2) CPLEX 7.5 com o método dual simplex;

${ }^{(3)}$ DSBU: Método dual simplex com busca unidimensional;

${ }^{(4)}$ FO: função objetivo.
} 
Observamos que, apesar do método dual simplex com busca unidimensional não se utilizar de nenhum procedimento anti-ciclagem, a soluçâo de cada problema foi cncontrada em número menor de iterações do que o CPLEX (exceto exemplar 5). Os autores também já haviam relatado que os principais pacotes de otimização resolvem todos os exemplos construídos sem ciclagem.

Nas duas próximas seções são descritos dois procedimentos anti-degeneração que adaptamos para o método dual simplex com busca unidimensional.

\subsection{Métodos Anti-Degeneração}

\subsubsection{Introdução}

Em problemas altamente degenerados, isto é. muitas soluções básicas com variáveis $\mathrm{em}$ seus limitantes, tendem a provocar no método tipo simplex o fenômeno de estagnação.

Podemos observar tal fenomeno quando, usando o método dual simplex com busca unidimensional, realizamos testes com alguns problemas da Netlib. A principio, pensávamos que a busca unidimensional fosse capaz de evitar ou, pelo menos, reduzir a estagnação, pois quando na seqüência os pontos de não-diferenciação se acumulassem em zero, a busca linear por partes fosse capaz de "saltar" os zeros inconvenientes por redefinir os limites das restrições ativas (por exemplo, $\boldsymbol{a}_{B_{i}}^{T} \boldsymbol{x}=d_{B_{i}}$ por $\boldsymbol{a}_{B_{i}}^{T} \boldsymbol{x}=e_{B_{1}}$, já que $\lambda_{B_{i}}=0$, solução degenerada, veja capítulo 3 ). Convém notar que um esforço grande foi despendido elaborando algoritmos e implementações para estas redefinições dos limites das restriçôes ativas (vale lembrar também (veja capítulo 3) que se $\lambda_{B_{i}}>0$

então $\boldsymbol{a}_{B_{i}}^{T} \boldsymbol{x}=d_{B_{i}}$ ou se $\lambda_{B_{i}}<0$ então $\boldsymbol{a}_{B_{i}}^{T} \boldsymbol{x}=\mathcal{c}_{\beta_{i}}$, mas se $\hat{\lambda}_{B_{i}}=0$ há uma indefinição que pode ser revista a qualquer momento, em particular na busca unidimensional, quando passos nulos são obtidos). Concluímos, ao final, que a busca unidimensional realiza estas redefinições, mas a estagnação persistiu. Isto nos motivou a rever a literatura sobre estagnação e adaptá-la ao método dual simplex com busca unidimensional.

\subsubsection{Método de Expansão}

Nesta seção apresentamos uma técnica desenvolvida por Gill et al. (1989) para tratar a estagnação no método simplex. Os autores realizaram experimentos computacionais com 53 problemas da Netlib c obtiveram uma melhora significativa no número de iterações do método simplex. 
Para o desenvolvimento do método de expansão de Gill et al. (1989), considere o método primal simplex (veja apêndice B).

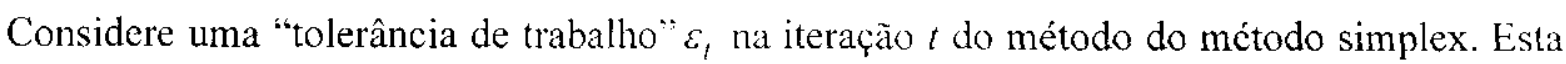
tolerância é ligeiramente incrementada antes de cada passo do método de simplex.

Escolhida a variável para entrar na base, digamos $x_{\varphi}$, e calculada as coordenadas básicas da direção primal simplex $\alpha_{q}=-\boldsymbol{B}^{-1} a_{N_{q}}$. o método de expansão inicia atualizando a tolerância de trabalho por: $\varepsilon_{t}=\varepsilon_{t-1}+\tau$. Com esta nova tolcrância calcula-se a seguinte razão:

$$
\delta^{*}=\min \left\{-\frac{x_{B_{i}}+\varepsilon_{i}}{\alpha_{i q}}, i \in B, \text { tal que } \alpha_{i q}<-\varepsilon_{z}\right\},
$$

$\mathrm{cm}$ que $\varepsilon_{z}$ é a tolerância para verificar se a i-ésima componente da direção primal simplex é menor do que zero. Note que $\delta^{*}>0$. Calcule também os valores que anulam as variáveis básicas,

$$
\hat{\delta}_{i}=\left\{-\frac{\hat{x}_{B_{i}}}{\alpha_{i q}}, i \in B . \text { ul que } \alpha_{i q}<-\varepsilon_{z}\right\} \text {. }
$$

Defina $\mathrm{S}$ o conjunto dos indices das razões em (5.2) menores do que $\delta^{*}$, isto é, $S=\left\{i \in B: \hat{\delta}_{i} \leq \delta^{*}\right\}$.

Escolha um índice $p \in S$ (que define o índice da variável que sai da base) tal que $\left|\alpha_{p q}\right| \geq\left|\alpha_{i q}\right| \forall i \in S$. Esta escolha opta pelo pivô maior $\left(\alpha_{p q}\right)$ quando há empate na escolha da variável que sai da base (Isto tende a melhorar a estabilidade numérica do método simplex).

Seja $O=\max \left\{\hat{\delta}_{p}, 0\right\}$. Observe que $\hat{\delta}_{p}$ é o passo necessário para zerar $x_{B_{p}}$. Até este ponto, 0 procedimento é conhecido como o Teste das Razóes de Harris (1972), desenvolvido para que o pivô scja mais bem escolhido, quando houver possibilidade. () procedimento de expansão assegura que o tamanho do passo (menor passo aceitável) seja sempre difcrente de zero e a função objetivo decresce, evitando a estagnação (mesmo que simbolicamente). Para isso, faça $\theta_{\min }=\frac{\tau}{\alpha_{p q}}>0$ e o tamanho do passo $\theta_{E}$ é determinado por: 


$$
\theta_{i}=\max \left\{\theta, 0_{\operatorname{man}}\right\}
$$

A seguir, o resumo do procedimento.

Algoritmo - Método de Expansão (inicio da iteração t):

Passo 0: INCREMENTO DA TOLERÂNCIA

Faça $\varepsilon_{l}=\varepsilon_{t-l}+\tau$;

Passo 1: PASSO EXPANDIDO

Calcule $\delta^{*}=\min \left\{-\frac{x_{B_{1}}+\varepsilon_{t}}{\alpha_{i q}}, i \in B\right.$, tal que $\left.\alpha_{i q}<-\varepsilon_{z}\right\}$;

Passo 2: PASSO PIVÔ

$p \leftarrow 0 ; \alpha_{\max } \leftarrow 0 ;$

Para $i=1$ ate $m$ faça

Se $\alpha_{i q}<-\varepsilon_{z}$ cntão

$$
\hat{\delta}_{i}=-\frac{x_{B_{1}}}{\alpha_{19}}
$$

se $\hat{\delta}_{i} \leq \delta^{*}$ e $\left|\alpha_{i q}\right|>\alpha_{\max }$ então

$$
\begin{aligned}
& p \quad i \\
& 0=\hat{\delta} \\
& \alpha_{\max }=\left|\alpha_{i q}\right| ;
\end{aligned}
$$

fim do se

fim do para

\section{Passo 3: PASSO NÃO NULO}

Faça $O_{\min }=\frac{\tau}{\alpha_{p q}}$ e determine $\theta_{k}=\max \left\{0,0_{\min }\right\}$ (tamanho do passo)

Se o passo usual para o maior clemento pivô é suficientemente maior do que zero, este é escolhido, senão um pequeno passo positivo é forçado. 
Como em qualquer iteração do método, a variável não básica é aumentada por uma quantidade positiva na direção que melhora a função objetivo logo o valor da função objetivo nunca retorna a um valor anterior.

No método dual simplex com busca unidimensional (DSBU) as razões anulam uma variável básica dual e podem ser ultrapassadas, promovendo uma troca no sinal da variável básica $\mathrm{e}$ conseqüentemente mudança na taxa de variação na lunção objetivo dual (veja capítulo 3). Implementamos um procedimento simples, para o qual obtivemos os melhores resultados.

No desenvolvimento original do DSBU. caso ocorra violação primal no limitante superior, as razões $\frac{\lambda_{B_{i}}}{\eta_{i q}}$ (veja o desenvolvimento no capitulo 3) são calculadas se $\lambda_{B_{i}}$ c $\eta_{i q}$ têm sinais opostos. Para o caso de ocorrer a violação prinal no limitante inferior, as razões são calculadas se $\lambda_{B_{i}}$ e $\eta_{i q}$ têm mesmo sinal.

A única mudança que realizamos no desenvolvimento original de DSBU foi calcular essas razões utilizando-se a tolerância de trabalho $\varepsilon_{t}$ (incrementado por 1e-7) a cada iteração do método e a busca unidimensional como definido no capitulo 3 faz a escolha de qual variável deixa base.

Tentamos outras variações neste procedimento para que tivéssemos um método de expansão como o original, como por exemplo, ao realizar a busca unidimensional, fosse levado em conta o tamanho do elemento pivô, mas não obtivemos bons resultados.

\subsubsection{O Método de Perturbação}

O método de perturbação, segundo Maros (2003), é um procedimento heurístico muito simples que pode ser eficiente se implementado com alguns cuidados.

Para cada posição degenerada, faça uma perturbação aleatória nas variáveis que são degeneradas mas que permaneçam factíveis após essa perturbação. Se uma variável degenerada deixa a base, atualize somente as posições que são degeneradas. Caso contrário um passo não nulo é realizado, neste caso restaure os valores das variáveis originais (elimine as perturbações feitas) antes de atualizar a base.

Para o método dual simplex com busca unidimensional, fizcmos apenas perturbações nas razões próximas de zero, iguais a num $\times \mathrm{e}-5$, em que num é gerado aleatoriamente no intervalo $1 \leq n u m \leq 9$ para cada posição nula. Com isso, eliminamos o passo nulo na direção dual simplex. 
Um outro método, também baseado em perturbações, é o método de Wolfe (1963) que é detalhado em Maros (2003). Ryan c Osborne (1988) mostraram que o método tem um ótimo desempenho $\mathrm{cm}$ alguns problemas de otimização combinatória. Este método foi estendido para o DSBU, sem que o efeito da estagnação fosse atenuado e, portanto, não discutiremos neste trabalho.

\subsection{Breve Revisão de Variantes do Método Simplex}

Nesta seção relatamos algumas variantes do método simplex com algumas características com ênfase na regra de Dantzig Normalizada, que foi estudada e adaptada para o método dual simplex com busca unidimensional. Para um estudo mais detalhado dessas variantes citadas $\mathrm{c}$ apresentadas a seguir, apontamos os trabalhos de Terlaky e Zhang (1993).

\subsubsection{Introdução}

Tipicamente, métodos tipo simplex caminham nas arestas do conjunto de soluções, de vértice em vértice, em busca da solução ótima. Esse movimento é chamado de pivoteamento, que é a operação básica em todos métodos simplex e consiste na mudança de base, em que a nova base difere da anterior exatamente em uma coluna (linha).

Este processo pode requerer muitos passos $\mathrm{cm}$ um poliedro (região factível) com muitas faces. Intuitivamente, um caminho mais direto passando pelo interior desse poliedro é atraente, já que existe a possibilidade de se chegar à solução ótima em poucos passos (Terlaky, 2000), procedimento que os métodos de pontos interiores exccutam.

\subsubsection{Considerações Sobre Métodos Tipo Simplex}

Terlaky (2000) considera três classes de métodos tipo simplex: métodos primais (soluções primais factíveis são percorridas), métodos dulais (soluções duais factíveis são percorridas) e métodos primal-dual exterior (soluções infactíveis).

A vasta literatura de otimização linear contém uma família expressiva de métodos simplex. $\Lambda$ flexibilidade inerente de escolher as variáveis que entram e saem da base permite desenvolver vários critérios para a escolha destas variáveis, como por exemplo, a regra de Dantzig Normalizada descrita na seção 5.4.5. 
A maioria dos desenvolvimentos nos últimos anos de técnicas e programação nos softwares de otimização linear, é de implementações de métodos tipo dual simplex, pois esses métodos são muitos eficientes, especialmente em resolver problemas de otimização combinatória (Terlaky, 2000 e Bixby 2001). Algumas variantes do método dual simplex podem ser encontrados em Dantzig (1963) e Terlaky e Zhang (1993).

Com relação à complexidade dos métodos tipo simplex, é sabido que, como relatado no capítulo 2, no estudo do pior caso as principais variantes do método simplex são exponencial (Klee e Minty, 1972), Goldfarb e Sit (1979) e Terlaky e Zhang (1993). Um fato curioso é que o método dual simplex resolve o problema de Klee e Minty em número polinomial de iterações (Murty, 1983). E outro exemplo exponencial é dado por Clausen (1979) e Murty (1983).

Havia um enorme 'abismo' entre a complexidade polinomial teórica e o desempenho na prática dos métodos tipo simplex (Todd, 2001). Era óbvio que nenhum método simplex implementado na prática usaria o caminho exponencial de Klee e Minty. De fato, os pacotes de otimização linear comercial resolvem o problema $\mathrm{cm}$ uma única iteração, assim como o DSBU. Fntão a necessidade de explicar a eficiência dos métodos simplex tornou-se cvidente (Todd, 2001). Para isto foi demonstrado que. sob certos modelos probabilísticos, o número médio esperado de iterações é linear (Borgwardt, 1987; Adller c Megiddo (1984).

Adler e Megiddo (1985) analisaram o número médio de itcrações realizadas pelo algoritmo simplex conhecido como método self-dual (Danztig, 1963). Eles mostraram que o número médio csperado de iteraçôes é limitado entre duas funções quadráticas da menor dimensão do problema $\mathrm{c}_{1}(\min (m, n))^{2}$ e $\mathrm{c}_{2}(\min (m, n))^{2}$ e sem nenhuma restriçào (exceto degeneração) no problema, $(m$ é o número de restrições e $n$ o número de variáveis).

A análise probabilística de algoritmos do tipo simplex cxige uma especificação na distribuição probabilística dos dados de entrada (lodd, 2001). Como por exemplo, distribuições esfericamente simétricas que foram utilizadas por Borgwardt (1982) e Smale (1983). Ambos autores trabalharam com algoritmos simplex paranétricos (Shamir, 1987) e, isto é aparentemente uma propriedade chave para realizar uma análise probabilística (Todd, 2001).

O trabalho de Borgwardt (1982) apresentou un limitante superior que é $O\left(n^{4} m^{1 / n-1}\right)$ para um modelo restrito e o algoritmo resolve somente problemás de subclasses particulares.

Smale (1983) analisou um algoritmo menos restrito e mostrou que existe uma constante $\mathrm{K}$ tal que o número esperado de iterações é menor do que $\mathrm{K}(\ln n)^{m(m-1)}$.

Assim, segundo Adller e Megiddo (1984). ten sido um desafio para os matemáticos confirmar teoricamente o bom desempenho de algoritnos do tipo simplex para a otimização linear. 
Um outro desenvolvimento relevante, de novo especialmente eficiente quando resolve problemas de otimização combinatória (Terlaky. 2002). é a implementação da regra de Dantzig Normalizada (Forrest e Goldfarb. 1992; Bixby. 1994). Além desses trabalhos o estado da arte computacional dos métodos tipo simplex pode ser encontrado nos artigos de Bixby (1994), Maros e Mitra (1996) e Maros (2003).

\subsubsection{Métodos Primal-Dual Exterior (Criss-cross)}

Optamos por apresentar os métodos primal-dual exterior, conhecido na literatura inglesa por Criss-cross, pois pertencem a uma classe de métodos tipo simplex diretamente relacionado a outras variantes simplex que têm propriedades teóricas interessantes que comentamos na próxima seção.

Esses métodos podem ser inicializados por uma solução básica qualquer, $\mathrm{cm}$ seguida a otimalidade é verificada. Caso a solução bésica não seja a ótima, uma variável primal (dual) infactível é escolhida para sair (entrar) da base. Este processo é repetido até a solução ótima (Terlaky, 1985) e (Fukuda e Terlaky, 1997) ou identilicando a infactibilidade primal ou dual.

Apresentaremos aqui uma variante finita de um método primal-dual exterior (Terlaky, 1985; Fukuda e Terlaky, 1997) conhecido como a do menor índice, que é extremamente simples e resolve problemas de otimização linear.

$\Lambda$ s vantagens de métodos primal-dual exterior é que eles podem ser inicializados com qualquer solução básica e assim são métodos de uma só fase: não preservam factibilidade, logo nenhum trabalho é feito para garantir esta propriedade; somente os sinais das variávcis são verificados, sem considerar a magnitude delas.

$\Lambda$ seguir, damos o algorimo para esta variante do menor índice, desenvolvida por Terlaky (1985).

Algoritmo primal-dual exterior do menor indice

\section{Inicialização}

Seja $\boldsymbol{B}$ uma base inicial arbitrária.

\section{Enquanto pare $=$ falso faça}

Se $\boldsymbol{x}_{B} \geq 0$ e $c_{N} \geq 0$ então pare--verdade

A solução atual é ótima 


\section{Senão}

\section{Calcule:}

$$
\begin{aligned}
& p=\min \left\{i \text { tal que } x_{j}<0, i \in B\right\} ; \\
& q=\min \left\{j \text { tal que } c_{j}<0, j \in N\right\} ; \\
& r=\min \{p, q\} ;
\end{aligned}
$$

\section{Se $r=p$ então}

Se $p$-ésima variável primal é não negativa

Pare: o problema primal é infactível;

Senão

$$
\operatorname{Seja} q-\min \left\{j \in N, \alpha_{p}=\left(\boldsymbol{B}^{-1} \boldsymbol{a}_{j}\right)_{p}<0\right\} ;
$$

Senāo $(r=q)$

Se $q$-ésima variável dual é não positiva

Pare=verdadé: o problema dual é infactível;

\section{Senão}

$$
\text { Seja } p \cdot \min \left\{i \in B, \alpha_{i, f}=\left(\boldsymbol{B}^{-1} \boldsymbol{a}_{q}\right)_{i}>0\right\} \text {; }
$$

Realize a troca de base: $B=B \cup\{q\}\{p\}$;

Este é um método finito e a prova desta propriedade encontra-se em (Terlaky e 7hang, 1993). A demonstração de que esse método é linito permite flexibilidade na seleção do elemento pivô, embora a descrição acima pareça ser rígida na escolha das variáveis que entram e saem da base.

Uma outra variante para esse método é obtida pela regra de escolha da variável: primeira que entra é a primeira que sai. Para mais detalhe sobre esta regra, veja Zhang (1999).

$\Lambda$ pesar da elegância e simplicidade segundo Terlaky (2000) observa que os métodos de primal-dual exterior não são eficientes na prática c̀ optamos por não implementá-los

\subsubsection{Comentários Sobre Outras Variantes Simplex}

Relacionado com os métodos primal-dual exterior. Anstreicher e Terlaky (1994) publicaram o algoritmo do tipo simplex MBU (Monotonic Build-Up), que tem propriedades teóricas interessantes. Os autores apresentam a versão primal e dual do algoritmo MBU. 
A versão MBU-primal inicia com uma solıção básica primal factível e algum custo reduzido negativo. A regra produz uma seqüencia de iterações, tal que todos custos reduzidos que eram originalmente não negativos permanecem nĩo negativos. A regra então constrói monotonicamente (estritamente crescente) uma base dual facivel, e assim uma base ótima. Veja detalhes em Anstreicher e Terlaky (1994).

Existem duas propricdades interessantes no método MBU. Primeiro, há dois testes da razão a cada iteração - ambas as variáveis, de entrada e de saída, sâo determinadas por estes testes. A factibilidade dual (custos reduridos não-negativos) é preservada durante o algoritmo, devido ao segundo teste da razão, enquanto a primal factibilidade é temporariamente perdida e recuperada.

Como o método produz uma seqüência de iterações, que resulta $\mathrm{em}$ bases intermediárias que não são primal nem dual factíveis, pode ser visto como um método de pontos exteriores (Paparrizos, 1996).

É curioso notar que os métodos de Paparrizos (1989) e Goldfarb (1983) são casos especiais do método MBU, conhecidos por EPSA (Exverior Point Simplex Algorithm) sâo exponenciais no pior caso. E, como são casos especiais do MBI l. este é também exponencial no estudo do pior caso.

Esforços têm sido feitos para relaxar a necessidade de factibilidade (primal ou dual) nos métodos tipo simplex (Terlaky, 2002). O primciro passo foi o método simplex paramétrico self-dual de Dantzig (1963).

Como rclatamos na seçĩo anterior, os métodos primal-dual exterior de Zionts (1969). Terlaky (1987) podem ser iniciados com qualquer base. Além disso, ao contrário do MBU ( $\Lambda$ nstreicher e Terlaky, 1994), se a factibilidade primal ou dual acontece durante os passos do algoritmo, é puramente acidental. No método de Zionts a factibilidade é preservada uma vez obtida. mas no método (finito) de Terlaky não ocorre esta situação.

Existem diversas variantes de métodos tipo simplex e também para os métodos de pontos interiores, como existem também algumas combinações de métodos tipo simplex com métodos de pontos interiores (MPI). Como por exemplo, utilizar inicialmente MPI e, em seguida, aplicar o método simplex (Luh e Tsaih, 2002). Segindo estes autores esta combinação reduz o número de iterações em aproximadamente $40 \%$ nos problemas tratados por eles.

Segundo Luh e Tsaih (2002) experimentos apontam que métodos de pontos interiores superam o método tipo simplex para problemas de grande escala (de’enas de milhares de variáveis) c uma investigação ainda interessante a ser examinada, segundo Luh e Tsaih (2002), é de como combinar as melhores características desses dois tipos de métodos para produzir um algoritmo eficiente para problemas de tamanho médio. Mas, esse melhor desempenho para os métodos de 
pontos interiores afirmado pelos autores depende certamente também das classes de problemas. como por exemplo, problemas de corte de estoque. com centenas de milhares de variáveis $c$ relativamente poucas restrições o método simplex com geração de colunas se mostra muito eficiente.

Embora haja alguns relatos sobre o mellor desempenho de métodos de pontos interiores, não conhecemos na literatura trabalhos que comparam e apontam esta superioridade, sobre os métodos tipo simplex, em classes bem detinidas de problemas de otimização linear. Assim, como relatado $\mathrm{cm}$ Bixby et al. (2000), cada tipo de método é superior $\mathrm{em}$ um número significante de exemplares importantes de otimização linear. $\Lambda$ identificação de classes de problemas, para as quais um ou outro tipo de método é mais bem adequado, persiste como tópico a ser investigado.

O trabalho de Anderson e Ye (1996) indica que al combinação de métodos de pontos interiores e métodos tipo simplex tem garantia de convergência teórica e prática c propuseram um método que resolve problemas de otimização linear em $\left(n^{11.5} L\right)$ iterações em que $L$ é o número de bits para armazenar os didos dos problemas. ou seja. un algoritmo polinomial. Outros experimentos computacionais que combinam esses dois tipos de métodos são encontrados $\mathrm{cm}$ Bixby et al. (1992).

Um trabalho recente é de Vieira c Lins (2005) que propuseram uma técnica de encontrar uma base inicial factível que estẹa próxima da base otima para o método primal simplex.

O método dos autores foi superior em relação ao método simplex usual, realizando $33 \%$ menos iterações. No entanto, cles trabalharam com problemas muitos pequenos (máximo de 50 restrições e 50 variáveis). gerados aleatoriamente de uma forma bem particular, não tendo apresentado o tempo computacional. İ bem provível que o tempo computacional seja alto pelo fato do método calcular inicialmente o ângulo entre o gradiente da função objetivo e o gradiente de todas as restrições $\quad\left(\theta=\arccos \frac{c^{\mathrm{T}} a^{\prime}}{\|c\|_{i} a^{\prime} \mid}\right)$ o que caracteriza um procedimento caro computacionalmente paril problemas de grande porte.

\subsubsection{Regra de Dantzig . Vormalizada}

Nos métodos tipo simplex há. en guril, uma flexibilidade de escolha para a troca de base. Por excmplo, no método primal simplex qualquer variavel com custo relativo negativo pode ser escolhida para "entrar na base", ou no méludo dual simplex. tanto o clássico como os dos tipos desenvolvidos no capítulo 3, pode-se escollher qualquer restrição primal violada para "dcixar a 
base". Desta forma algumas regras heurísticas para esta escolha foram desenvolvidas (já que em um algoritmo os passos têm de ser bem determinados)

A regra de Dantzig ou regra usual escolhe uma direção simplex que tem o coeficiente mais negativo com o desejo de uma redução signilicante no valor da função objetivo, no entanto, esta escolha negligência o fato que o comprimento de cada aresta que determina esta direção pode ser diferente. Portanto, a regra de Dantzig normalizada esculhe uma variável que tcm o coeficiente normalizado mais negativo, ou seja, fornece o quanto cada aresta contribui a função objetivo por unidade desta direção.

A regra de Dantzig Normalizada (steepest-edge rule) é conhecida desde os experimentos de Kuhn e Quandt (1963) e Wolfe e Cuttler (1963), porém. por causa de sua implementação direta, foi inicialmente rejeitada como impraticável por demandar alto tempo computacional. No entanto, Goldfarb e Reid (1977) publicaram o primeiro algoritmo do tipo simplex com a regra de Dantzig Normalizada praticável. Os autores reportaram testes computacionais preliminares, mostrando a superioridade do algoritmo simplex com esta regra em relação a regra usual de Dantzig, que ficou mais evidente no trabalho de Forrest e Goldfarb (1992).

Forrest e Goldfarb (1992) apresentam um estudo mais abrangente para esta regra e fornecem fórmulas recursivas para atualização do cálculo das normas das direçôes primal e dual simplex. scja na forma padrão ou não. Realizaram testes computacionais com alguns problemas da Netlib e concluíram que, em geral, o algoritmo simplex com ssta regra realiza menos iterações em relação a regra usual, além da redução do tempo computacional.

Considerando o método dual simplex clássico (isto é, método dual simplex com a regra de Dantzig), a escolha da variável dual que vai sair da base é definida pcla maior violação primal. Assim, $\eta_{j}=-\left(\boldsymbol{B}^{T}\right)^{-1} \tilde{\boldsymbol{e}}_{j}$, em que $\tilde{\boldsymbol{e}}_{j}$ ć a j-ésima coluna da matriz identidade, é a direção dual simplex e a j-ésima restrição é a mais violada. Por excmplo, no método dual simplex clássico: $x_{B_{i}}=\min \left\{x_{B_{i}}<0\right.$ tal que $\left.i=1, \ldots, m\right\} \mathrm{cm}$ que $\boldsymbol{x}_{k}=\boldsymbol{B}^{-1} \boldsymbol{b}$, ou cm DSBU: $j$ ć o índice associado ao $\min \left\{d_{N_{i}}-y_{N_{i}}, y_{N_{i}}-e_{N_{i}}\right\}$ em que $y_{N_{1}}=\boldsymbol{a}_{N_{1}}^{T} \boldsymbol{x}$ e $\boldsymbol{x}=\boldsymbol{B}^{-1} y_{B_{3}}$ e $\left(y_{B_{1}}=d_{B_{1}}\right.$ se $\lambda_{B_{1}} \geq 0$ e $y_{B_{1}}=e_{B_{1}}$ se $\left.\lambda_{B_{1}} \leq 0\right)$.

Como o custo relativo é dado por: $\nabla h(\hat{\lambda}) \eta_{j} \cdot(\nabla h(\hat{\lambda})$ é o gradiente da função objetivo dual na solução dual básica, o qual é constante no caso clássico, $\nabla h(\hat{\lambda})=\boldsymbol{b}^{T}$, já que $h(\lambda)=\boldsymbol{b}^{T} \lambda$, diferentemente do caso canalizado, veja capítulo 3). Portanto, o custo relativo depende da norma da 
direção simplex e, se quisermos a menor derivada direcional devemos então normalizar a direção simplex e devemos escolher o indice $j$ tal que: $V h(\hat{\hat{\lambda}}) \frac{\eta_{i}}{\left\|\eta_{i}\right\|}=\min \left\{\nabla h(\hat{\lambda}) \frac{\eta_{i}}{\left\|\eta_{i}\right\|}, i=1, \ldots, m\right\}$.

Porém, se por um lado $V h\left(\hat{\lambda}_{3}\right) \eta$ é lacilmente calculado pela violação de uma restrição primal, sem a necessidade explícila de $\eta_{i}$. cujo cálculo envolve a resolução de um sistema linear: $\eta_{t}=-\left(\boldsymbol{B}^{\gamma}\right)^{\prime} \tilde{\boldsymbol{e}}_{i}$ por outro lado, $\nabla h(\hat{\lambda}) \frac{\eta_{t}}{\|\left.\eta_{i}\right|_{1}}$ necessita explicitamente de $\eta_{i}$, tornando a regra de Dantzig normalizada computacionalmente cara (isto inviabilizou as primeiras implementações).

Entretanto, Forrest c Goldfarb (1992) derivam förmulas recursivas para atualizar as direções primal e dual simplex de uma iteraçào para al outlai.

Para o desenvolvimento das fómulas de atualização das direções dual simplex, considere que a variável de índice $q$ substitui a variável de indice p na base. Sabemos $\bar{B}^{-1}=\boldsymbol{E}^{1} \boldsymbol{B}^{-1}$, em que $B^{1}$ é a base atual e $\dddot{B}^{1}$ é a base na iteração subseqüiente e $E^{-1}$ é obtida pelo produto de matrizes elementares que correspondem à operação de pivoleamento e que a nova base pode ser escrita em função da base anterior por: $\bar{B} \cdot \boldsymbol{B}_{i}\left(\boldsymbol{a}_{4}-\boldsymbol{a}_{n}\right) \tilde{\boldsymbol{e}}_{i}$. Assim. de umil itcração para outra, a inversa dil nova base é dada por:

$$
\bar{B}^{-1}=B^{-1}-\frac{w^{\prime}-\bar{c}_{p}}{w_{p}} \ddot{e}_{\eta}^{\prime} \boldsymbol{B}^{\prime} . \quad \text { em que } \boldsymbol{w}=\boldsymbol{B}^{-1} \boldsymbol{a}_{q}
$$

Para o desenvolvimento das Cómulas de recortêneia ou atualização para a regra de Dantzig Normalizada (Coldfarb a Reid. 1977), considere o problema otimização linear no formato geral (3.1) e o mélodo dual simplex com busca unidimensional com as mesmas hipóteses do capítulo 3.

Para o método dual simplex especializado ao problema (3.1), a escolha da variável que vai entrar na base, segundo a regra de Dantzigg Normalizada. é delinida pela maior violação primal no limite inferior ou superior (que são os custos relativos para o método dual) dividida pela norma da direção dual simplex. ou seja. escolhe um índice $j$ não básico que fornece a maior das seguintes razões em valor absuluto: $\begin{gathered}d,-\hat{y}, \\ \left\|\eta_{l}\right\|\end{gathered}$

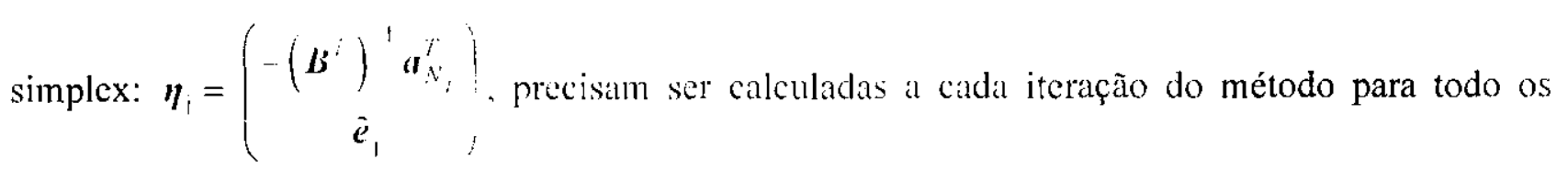
indices não básicos. 
Com o propósito de atualizar as direções duais simplex de uma iteração para outra, considere que a variável de índice q $(q \in\{1, \ldots, m-n\})$ substitui a variável de índice $p$ $(p \in\{1, \ldots, n\})$ na base. Sabemos que de uma iteração para outra. a inversa da nova base transposta é dada por:

$$
\left(\bar{B}^{1}\right)^{\prime}=\left(\boldsymbol{B}^{1}\right)^{\prime}-\frac{\left(w^{\prime}-\hat{\mathfrak{e}}_{p}\right)}{w_{p}} \tilde{\boldsymbol{e}}_{p}^{T}\left(\boldsymbol{B}^{-1}\right)^{\prime}
$$

cm que $B^{-1}$ é a base atual, $\bar{B}^{\prime}$ a base na itcração subseçüente e $w=\left(B^{l}\right)^{T} \boldsymbol{a}_{q}^{T}$. A identidade acima decorre de: $(\overline{\boldsymbol{B}})^{\mathrm{T}}=(\boldsymbol{B})^{\mathrm{T}}+\left(\boldsymbol{a}_{q}-\boldsymbol{a}_{p}\right) \tilde{\boldsymbol{e}}_{p}^{T}$.

As novas direções duais simplex são dadas por:

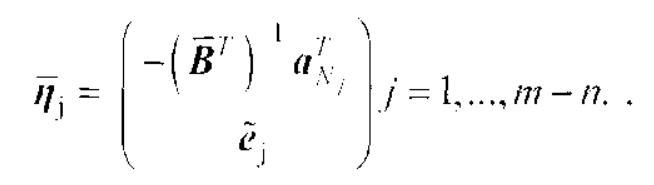

Para obter as novas normas das direções cm função das anteriores, desenvolvemos estas normas ao quadrado, pois facilita a manipulação algébrica.

Portanto, $\left\|\overline{\boldsymbol{\eta}}_{B_{i}}\right\|^{2}=\boldsymbol{a}_{N_{J}} \overline{\boldsymbol{B}}^{-1}\left(\overline{\boldsymbol{B}}^{T}\right)^{\prime} \boldsymbol{a} !$. Lsando (5.3) temos que:

$$
\left\|\overline{\boldsymbol{\eta}}_{B_{1}}\right\|^{2}=\left\|\boldsymbol{\eta}_{B_{3}}\right\|^{2}-\bar{\alpha}_{j} \boldsymbol{a}_{N_{1}} \boldsymbol{B}^{-1}\left(\boldsymbol{w}-\tilde{\boldsymbol{e}}_{p}\right)-\bar{\alpha}_{i}\left(w-\tilde{\boldsymbol{e}}_{p}\right)^{T} \boldsymbol{B}^{T} \boldsymbol{a}_{N_{j}}^{T}+\left(\bar{\alpha}_{j}\right)^{2}\left(w-\tilde{e}_{p}\right)^{\mathrm{T}}\left(\boldsymbol{w}-\tilde{\boldsymbol{e}}_{p}\right) .
$$

em que $\bar{\alpha}_{j}=\frac{\alpha_{j}}{\alpha_{q}}$, com $\alpha_{j}=\tilde{e}_{p}^{T} B^{-7} a_{N_{i}}^{T}$. Pela delinição de $w$,

$$
\bar{\alpha}_{t}=\frac{\tilde{e}_{p}^{T} B{ }^{\eta} \boldsymbol{a}_{N_{j}}^{r}}{w_{q}}
$$

Logo,

$$
\begin{aligned}
& \left\|\bar{\eta}_{B_{j}}\right\|^{2}=\left\|\eta_{B_{i}}\right\|^{2}-2 \bar{\alpha}, a_{Y}, B^{-1}\left(w-\tilde{e}_{p}\right)+\left(\bar{\alpha}_{j}\right)^{2}\left(1+w^{2} w-2 \bar{e}_{p}^{T} w\right) \\
& \left\|\overline{\boldsymbol{\eta}}_{B_{i}}\right\|^{2}=\left\|\boldsymbol{\eta}_{B_{i}}\right\|^{2}-2 \bar{\alpha}_{i} a_{x_{1}} B^{-1} \boldsymbol{w}+\left(\bar{\alpha}_{j}\right)^{2}\left(1+\boldsymbol{w}^{T} \boldsymbol{w}\right) .
\end{aligned}
$$


Agora, se denotarmos $\boldsymbol{v}=\boldsymbol{B}^{-1} \boldsymbol{w}$ e $\gamma_{j}=\left\|\left.\boldsymbol{\eta}_{1}\right|^{2}=1+\right\| \boldsymbol{\eta}_{B_{i}} \|^{2}$, então os quadrados das normils das novas direções duais simplex, são dadas por:

$$
\bar{\gamma}_{j}=\gamma_{j}-2 \bar{\alpha}_{i} a_{j,}+u_{i} \ddot{\gamma}_{4} \quad j=1, \ldots, m-n, \quad j \neq q .
$$

Se $j=q$, temos:

$$
\bar{\gamma}_{q}=\frac{\gamma_{q}}{\alpha_{q}^{2}}
$$

Observe que na equação (5.4) existem dois cálculos a fazer, o qual não estaria disponível se estivéssemos cxccutando uma iteração do método simplex com a regra de Dantzig: resolver o sistema $\boldsymbol{v}=\boldsymbol{B}^{-1} \boldsymbol{w}$ e o produto interno das linhas não básicas da matriz $\boldsymbol{A}$ com o vetor $\boldsymbol{v}$.

Segundo Forrest e (joldlarb (1992), devido alo cálculo inicial dos quadrados das normas das direções e depois suas atualizações com esse cálculo extra, leva a um aumento de tempo em tomo de $8 \%$ a mais por iteração do que o método simplex com a regra de Dantzig na implementação dos autores.

Tendo também como ponto de partida a identidade (5.3), de uma mancira similar esta técnica pode ser aplicada no cálculo das novas direções primal simplex.

Os autores trabalharam com alguns problemas da Netlib que em média tiveram 3412 linhas e 12492 colunas, sendo que o maior deles foi de $10662 \times 76090$. Para os 20 problcmas utilizados pelos autores, o tempo (em horas) para resolvê-los usando o algoritmo de Dantzig primal e dual foi respectivamente de $110,19 \mathrm{~h}$ e $177.78 \mathrm{~h}$, enquanto o algoritmo primal e dual proposto foi respectivamente de $4,89 \mathrm{~h}$ e $6,36 \mathrm{~h}$. Uma diferença muito impressionante. Excluindo cinco problemas en que o número de iteraçòes foi expressivamente alto, a média para o algoritmo de Dantzig foi $6,3 m$ enquanto o algoritmo com a regra de Dantzị Vormalizada realizou em média $1,9 m$ iterações. Um exemplar desses cinco problemas é o AIR2 (Netlib) com 5654 linhas, 8730 colunas e 271082 elementos diferentes de zero. () método primal simplex com regra de Dantzig Normalizada resolveu o problema com 24.158 iterações em 4.294 .9 segundos ao passo que o método primal simplex com a regra de Dantzig (primal) réalizou 1.345.993 itcraçốes em 195.207,0 segundos.

Portanto, o variante simplex com a regra de Dantzig Normalizada foi claramente superior as método simplex coma regra de Dantzig, para resolver alguns problemas difíceis de otimizaçio linear de grande porte da Netlib. 
Motivado principalmente pelo trabalho de Forrest e Goldfarb (1992) e no ótimo desempenho do método dual simplex com busca unidimensional comparado alo pacote CPLEX, rclativo ao número de iterações (Sousa, 2000), implementamos a regra de Dantzig Normalizada para o DSBU, para resolver alguns problemas gerados alcatoriamente de pequeno porte. Os resultados computacionais para os problemas que resolvemos se encontram no capitulo 7, os quais entretanto não revelam um bom desempenho para a regra de Dantzig . Vormalizada, evidenciando-se assim, uma classe de problemas em que esta regra tem um desempenho similar a regra usual de Danztig. Este scrá um tópico a ser mais bem investigado no futuro. 


\section{Capítulo 6 - Esparsidade e Estrutura de Dados}

Neste capítulo apresentamos as estruturas de dados que utilizamos em nossas implementações visando um melhor desempenho para o método dual simplex com busca unidimensional.

\subsection{Introdução}

Esparsidade é simplesmente a característica mais importante para os problemas de otimização linear de grande porte, o que torna viável resolvê-los em tempo aceitável. Uma questão lundamental para o sucesso de uma implementação do método simplex é como os dados são armazenados e usados no algoritmo de solução. Isto aumenta a necessidade de utilizar uma estrutura de dados apropriada.

Não existe uma estrutura de dados que seja a melhor para todos os propósitos. De fato, estrutura de dados deve ser adequada às operações a serem realizadas.

Existem dois tipos de estrutura de dados: a estática, que não muda sua estrutura durante as execuções e a dinâmica, que altera seu tamanho de acordo com a necessidade.

Não é surpresa que as estruturas dinâmicas sejam mais complicadas e os acessos individuais dos elementos consuma mais tempo do que as estruturas cstáticas. No entanto, ambas desempenham bem o seu papel. É importante utilizar também estruturas apropriadas para armazenar e acessar 
informações extras, como tipo e status de variáveis (básica, factível, etc.), limitante inferiores e superiores, vetores básicos, solução básicas, etc.

Nas próximas seções apresentamos as estruturas utilizadas no método dual simplex com busca unidimensional para cada técnica (heurísticas de decomposição e atualização da base, método do gradiente bi-conjugado, veja capítulo 3 ).

\subsection{Estrutura de Dados sugerida por Reid (1982)}

Para implementar de forma eficiente as heurísticas de decomposição e os métodos de atualização da base descritos no capítulo 3, foram utilizadas listas duplamente encadeadas sendo que o acesso a cada elemento da matriz pode ser feito tanto por linha quanto por coluna. Esta estrutura foi sugerida por Reid (1982) e amplamente estudada e implementada conjuntamente com Silva (2002), por fazer parte deste trabalho.

Assim. para armazenar uma matriz esparsa (seja a matriz de restrições ou a base) foram usados 2 vetores de ponteiros (rows e cols) alocados dinamicamente, cujos tamanhos são, respectivamente, o número de linhas $(n r)$ e o número colunas $(n c)$ da matriz. Um destes vetores contém ponteiros que apontam para listas (list) duplamente encadeadas que representam as linhas da matriz e o outro vetor, também contém ponteiros que apontam para listas duplamente encadeadas que representam as colunas da matriz. Estas listas, por sua vez, contêm ponteiros que apontam para o primeiro elemento não nulo de cada linha ou coluna (head) e um inteiro (count) que indica quantos elementos não nulos existem na linha ou coluna. E finalmente, cada elemento não nulo da matriz é representado por um nó (list_element) que contém um ponteiro que aponta para o elemento anterior (prev), um ponteiro que aponta para o próximo elemento (next), um ponteiro para o valor do elemento (value) e um inteiro (pos) que guarda a posição que o elemento ocupa na linha ou coluna.

Na Figura 6.1, temos a representação gráfica desta estrutura de dados accssando os elementos por coluna: 


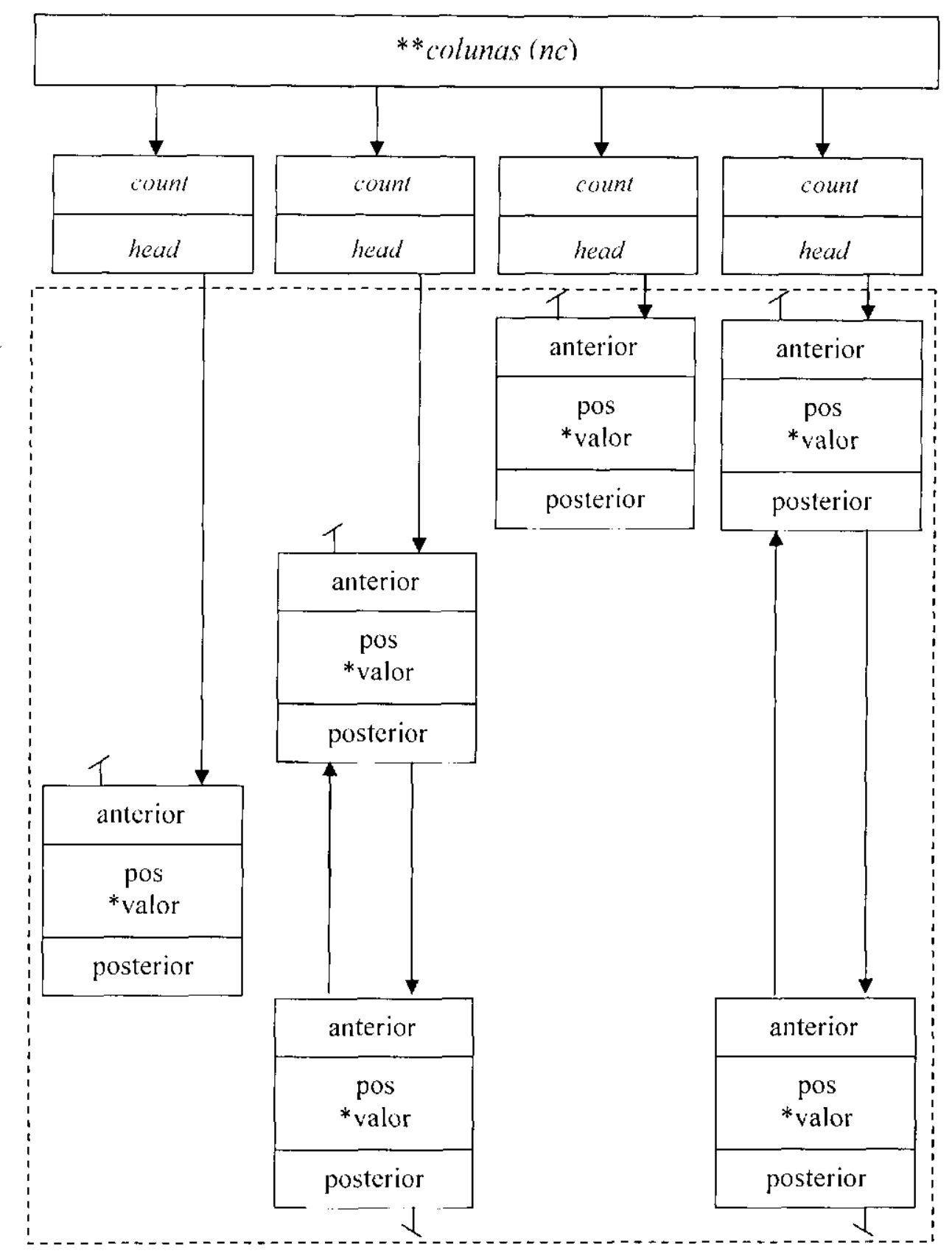

Figurara 6.1 - Representação da matriz acessando os elementos por coluna

Já a representação gráfica da estrutura de dados quando o acesso aos elementos é feito por linha é scmelhante à Figura $6.1 \mathrm{com}$ pequenas alterações no vetor de ponteiros e no nó.

\subsection{Estrutura de Dados para o Método GBC}

Utilizamos inicialmente, a estrutura de dados sugerida por Press et al. (1992) para o método do gradiente bi-conjugado ( $\mathrm{GBC}$, veja capítulo 3) e incorporamos à implementação do método dual 
simplex com busca unidimensional. Ressalta-se que esta estrutura serve somente para o armazenamento de matrizes quadradas. Assim, cla foi utilizada inicialmente somente para armazenar a matriz básica para aplicar o método $\mathrm{GBC}$ c para então verificar se o método convergia na resolução dos sistemas lincares, fato que foi confirmado.

A primeira estrutura é um tipo de esquema de armazenamento indexado (Press et al, 1992), que armazena somente os elementos não nulos da matriz, junto com uma informação auxiliar suficiente para determinar a posição de um elemento na matriz.

Assim, para representar uma matriz $\boldsymbol{A} \boldsymbol{I}$ de dimensão $n \times n$, o esquema chamado de indexação por linhas utiliza dois vetores unidimensionais, sa e $i j$ a. O primeiro deles armazena os valores de cada elemento da matri\% e o segundo armazena números inteiros. Apresentamos a seguir, um resumo de como é este esquema.

1. As primeiras $n$ posiçōes do vetor sa armazenam os elementos da diagonal principal da matriz A1 na ordem em que cles aparecem;

2. A posição $n+1$ do vetor sa é um valor qualquer, aqui denotado por $\otimes$;

3. As posições maiores que $n+1$ do vetor sa armazenam os demais elementos (fora da diagonal) da matriz, percorrendo-a por linha;

4. $\Lambda$ primcira posição do vetor i ja armazena sempre o valor $n+2$ :

5. As posições 2 até $n$ do vetor i ja armazenam os índices do vetor sa os quais indicam o início de armazenagem dos elementos (fora da diagonal) da linha 1 até linha $n$. Ou scja, essas posiçõcs apontam para o início de cada linha no vetor sa;

6. A posição $n+1$ do vetor $i j$ a é o último índice do vetor sa adicionando 1:

7. As posições maiores que $n+1$ do vetor ija armazenam o número da coluna em que os elementos fora da diagonal pertencem, percorrendo a matriz por linha.

Para um exemplo deste esquema de armazenagem, considere a seguinte matriz esparsa:

$$
\boldsymbol{A} I=\left(\begin{array}{cccc}
-2 & 0 & 0 & 1 \\
7 & 0 & 5 & 0 \\
3 & 0 & 1 & 0 \\
0 & -4 & 0 & 3
\end{array}\right)
$$


Então, temos que os vetores i ja e sa dados pela Figura 6.2.

\begin{tabular}{|l|c|c|c|c|c|c|c|c|c|c|}
\hline indice $[i]$ & 1 & 2 & 3 & 4 & 5 & 6 & 7 & 8 & 9 & 10 \\
\hline $\mathrm{ij \textrm {a } [}[i]$ & 6 & 7 & 9 & 10 & 11 & 4 & 1 & 3 & 1 & 2 \\
\hline $\mathrm{sa}[i]$ & -2.0 & 0.0 & 1.0 & 3.0 & $\otimes$ & 1.0 & 7.0 & 5.0 & 3.0 & -4.0 \\
\hline
\end{tabular}

Figura 6.2 - A matriz Al representada pelos vetores i ja e sa

Notc que, de acordo com as regras de armazenamento, o valor de $n$ (isto é 4) é i ja[1] - 2, e o tamanho (número de posições requisitadas para armazenagem) de cada vetor é ija[ija[1]-1]-1, isto é, 10. O elemento da diagonal na linha $i$ é dado por sa[i], e os elementos fora da diagonal nesta linha estão em $\mathrm{s} a[\mathrm{k}], \mathrm{k}=i j a[i]$ a $i j a[i+1]-1$. Por cxcmplo, considere a linha 2, o elemento da diagonal está em $s a[2]=0$, e os elementos fora da diagonal estão em $s a[7]=7 \mathrm{c}$ sa[8] $=5$, pois i ja[2]=7 e i ja[3]-1=8. Além disso, ija [7]=1 e i ja [8]=3 definem as colunas destes elementos.

Segundo Press et al (1992), o uso mais importante do armazenamento utilizando indexação por linhas de uma matriz quadrada é multiplicá-la por um vetor, operação principal no algoritmo do gradiente bi-conjugado. Mas, como precisávamos utilizar uma estrutura de dados que fosse capaz de armazenar não somente matrizes quadradas, e também adequada tanto para o método do gradiente bi-conjugado e quanto para as operações do método dual simplex com busca unidimensional, utilizamos a estrutura de dados sugerida por Maros (2003) que será apresentada na próxima seção, mas que já conhecida desde o livro de Duff et al em 1986.

\subsection{Uma Estrutura de Dados para o Método Simplex}

A estrutura de dados sugerida por Duff et. al (1986) e Maros (2003), não requer que a matriz em questão seja quadrada e pode ser utilizada também nas rotinas que compõem o método do gradicnte bi-conjugado.

$\Lambda$ maneira de armazenar uma matriz (Maros, 20003) não necessariamente quadrada é armazená-la como uma coleção de vetores colunas (linhas). Os elementos não nulos das colunas (linhas) podem ser armazenados consecutivamente colocando os índices (índices das linha) no vetor rind e os elementos no vetor val. A posição inicial (começo) de cada coluna deve ser guardada $\mathrm{em}$ um vetor, digamos cbeg. Se tivermos $n$ colunas, precisamos de $n+1$ posições, o último aponta 
para a primeira posição depois do último elemento da última linha. Desta forma o tamanho da coluna $j$ (cm termos de não-nulos) é clen $[j]=\operatorname{cbeg}[j+1]-c b e g l j]$. Em geral, o vetor clen não é necessário. No entanto, se as colunas não estão armazenadas consecutivamentc, é nccessário o uso deste vetor. Neste caso, a coluna é acessada pelos vetores cbeg e clen. Utilizar o vetor clen torna a cstrutura mais flexívcl. Alternativamente para o término das colunas, o vetor cend[j] pode ser usado, o qual dá uma flexibilidade similar.

Considere a matriz $\boldsymbol{A} 2$ abaixo como exemplo,

$$
\boldsymbol{A} 2=\left[\begin{array}{ccccc}
-1 & 0 & 0 & 2 & 0 \\
0 & 0 & -1 & 0 & 0 \\
1 & -1 & 0 & 0 & 2 \\
0 & 0 & 0 & 4 & -1 \\
0 & 0 & 0 & -3 & 1
\end{array}\right]
$$

Para a matriz A2 armazenada como uma colcção de vetores colunas esparsos, temos que os vetores que a determinam são dados pela Figura 6.3 .

\begin{tabular}{|l|c|c|c|c|c|c|c|c|c|c|}
\hline indice $[i]$ & 1 & 2 & 3 & 4 & 5 & 6 & 7 & 8 & 9 & 10 \\
\hline $\operatorname{cbeg}[i]$ & 1 & 3 & 4 & 5 & 8 & $(11)$ & & & & \\
\hline $\operatorname{clen}[i]$ & 2 & 1 & 1 & 3 & 3 & & & & & \\
\hline $\operatorname{rind}[i]$ & 3 & 1 & 3 & 2 & 4 & 1 & 5 & 3 & 4 & 5 \\
\hline $\operatorname{val}[i]$ & 1.0 & -1.0 & -1.0 & -1.0 & 4.0 & 2.0 & -3.0 & 2.0 & -1.0 & 1.0 \\
\hline
\end{tabular}

Figura 6.3 - $\Lambda$ matriz A2 representada por uma coleção de vetores coluna

O elemento cbeg[6]=11 está entre parênteses para indicar que este elemento do vetor cbeg é necessário somente se o vetor clen não é usado. Note que algumas colunas não estão em ordem. ou seja, os índices dos elcmentos não estão em ordem ascendente. Observe em cbeg $\lfloor i\rfloor=k$, que $k$ define a posição no vetor val $\mathrm{e} i$ o início da coluna $i$ da matriz. Por exemplo, para sabermos onde começa a coluna 4 temos que cbeg[4]=5, ou seja, a coluna começa na posição 5 do vetor $\mathrm{r}$ ind $\mathrm{c}$ val, com o clemento 4.0 armazenado como primeiro desta coluna.

Esta estrutura de representação é estática, assim seu principal problema torna-se cvidente quando um novo elemento precisa ser inserido ou eliminado. Mas, para a implementação do método 
dual simplex com busca unidimensional utilizando o método do gradiente bi-conjugado, ela é adequada, pois na resolução dos sistemas lineares, não ocorre a inserção de um novo elemento e nem a eliminação.

Esta estrutura de coleção de vetores coluna (linha) é, segundo Saad (1994) uma variação da estrutura de dados conhecida como compressed sparse column (row) a qual é composta por 3 vetores e armazena os clementos por coluna (linha) sem se preocupar com a posição que cles ocupam dentro de cada coluna (linha).

Saad (1994) descnvolveu um aplicativo computacional livre para se trabalhar com diversos tipos de matrizes esparsas denominado sparskit. Este aplicativo foi feito $\mathrm{cm}$ linguagem de programação Fortran e fornece rotinas para converter estruturas de dados, esboçar a estrutura de matrizes, realizar operações elementares de álgebra linear e etc.

Embora um dos objetivos do sparskit, segundo o autor, é fornecer uma ferramenta que facilita a troca de programas e dados entre os pesquisadores. não podemos utilizar tal ferramenta devido à incompatibilidade da linguagem de programação (pois implementamos em linguagem C), mas que pode ser estudado futuramente.

\subsection{Considerações na Implementação}

Para armazenar as restrições (matriz $N$ ) e a matriz base (matriz $\boldsymbol{B}$ ) na forma (3.1), (veja ligura 6.4) foi utilizada a estrutura de dados sugerida por Maros (2003), o método GBC foi implementado inicialmente com a estrutura de dados sugerida por Press et al. (1992) para verificação inicial da convergência, mas como precisávamos adotar uma estrutura única que atendesse as operações de cada parte dos métodos utilizados, decidimos trabalhar com a estrutura de dados sugerida por Maros (2003) $\mathrm{cm}$ todas as rotinas da implementação do método dual simplex com busca unidimensional. 


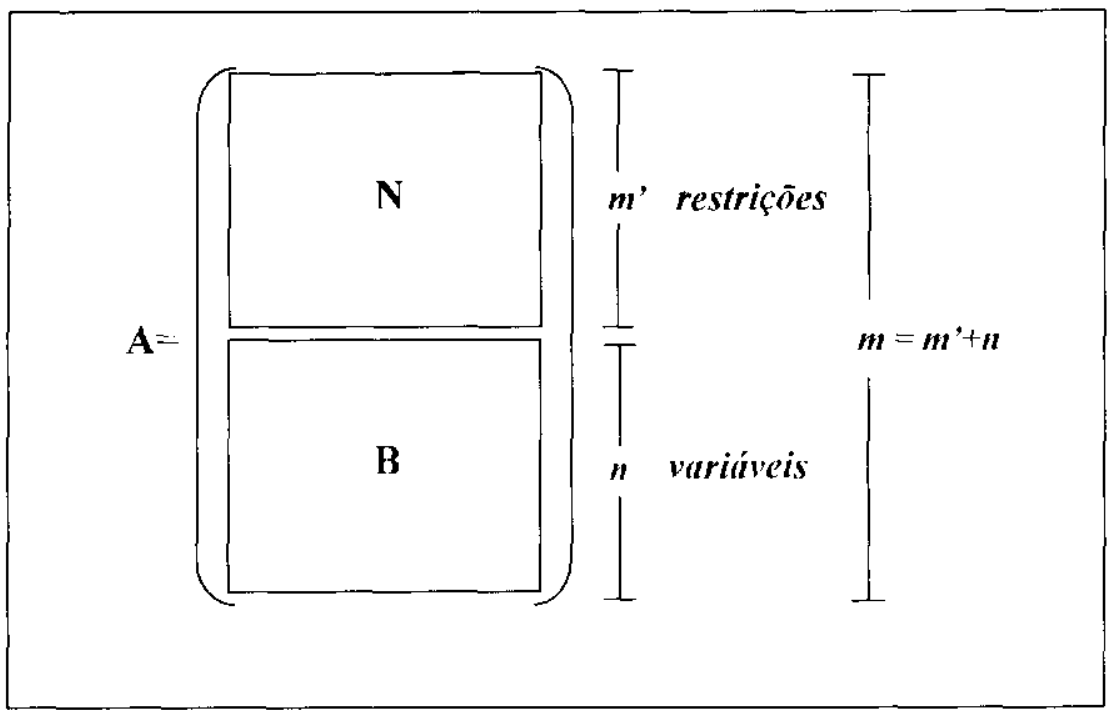

Figura 6.4-Representação da matriz na forma geral

Então, para que a implementação do método dual simplex ficasse com uma só estrutura, primeiramente o método do gradiente bi-conjugado (para resolver os sistemas lineares) foi adaptado para a estrutura sugerida por Maros (2003), caso contrário, teríamos uma implementação com rotinas de conversão de estruturas a cada iteração do método simplex, o que seria um procedimento muito caro.

Tendo em vista as operações que são realizadas no método do gradiente bi-conjugado c do DSBU, como por exemplo, a seleção da $i$-ésima linha da matriz de restrições aqui representada por $\boldsymbol{N}$ e a forma de atualizar a matriz base $\boldsymbol{B}$, foi escolhida a estrutura de dados que armazena os elementos da matriz por linha e não por coluna como está descrito na seção anterior

Lembrando que o método dual simplex com busca unidimensional resolve três sistemas lineares a cada iteração, sendo dois sistemas com a base transposta e o outro não (veja o algoritmo do capitulo 3). Assim, foi utilizada uma variável binária para identificar tal situação c fazer as alteraçõcs no método do gradiente bi-conjugado sem tornar tal procedimento caro.

Logo, tivemos que adaptar o gerador utilizado nos testes e o procedimento de leitura, para que na medida em que eram fornecidos os elementos da matriz de restrições (por linha) os vetores eram construidos. Em seguida, como no teste de otimalidade é preciso fazer o produto de uma linha da matriz de restrições por um vetor, então utilizamos a mesma rotina feita para o GBC já adaptado para esta estrutura.

Identificada a variável que entra na base pelo teste de otimalidade (restrição mais violada) determina-se a direção dual simplex que é a linha da matriz de restrições que fornece a maior 
violação primal (veja o algoritmo do capítulo 3). Como a armazenagem é realizada por linha, o vetor que determina a direção dual simplex é facilmente obtido.

Realizados os testes das razões e em seguida a busca unidimensional para determinar a variável que sai da base, faz-se à atualização da base. Para atualizar os três vetores que compõem a matriı base (primeiramente construídos no procedimento de inicialização) foi preciso construir um algoritmo bem estruturado para realizar as operações de inscrção e a remoção de uma nova coluna.

Para isto, foram tratadas situaçôes em que a coluna que substitui a outra na base, hora era uma linha da matriz de restrições (violação na matriz $N$ ) hora era uma linha da matriz identidade (violação primal nos limitantes das variáveis, matriz $\boldsymbol{B}$ ) e ainda, se o número de clementos para a linha que entra era maior, menor ou igual ao número de elementos da coluna que sai da base. Tudo isso com o intuito de se obter uma atualização mais econômica em termos de memória sem comprometer o tempo computacional. 


\section{Capítulo 7 - Experimentos Computacionais}

\subsection{Introdução}

Neste capítulo apresentamos os resultados computacionais para método dual simplex com busca unidimensional (DSBU), sendo que na seção 7.2 relatamos os resultados para as heurísticas de decomposição c os métodos de atualização. Na seção 7.3 apresentamos os experimentos com o método DSBU com as extensões na busca unidimensional propostas no capitulo 3. A seção 7.4 apresenta os resultados para problemas degenerados da Netlib e gerados aleatoriamente. Os resultados para a regra de Dantzig normalizada são apresentados na seção 7.5. E finalmente na seção 7.6 damos os resultados obtidos com o método do gradiente bi-conjugado para o DSBU.

\subsection{Heurísticas e Métodos de Atualização da Base}

Nesta scção, apresentamos os testes realizados com as heurísticas de decomposição: Grau Mínimo e Markowitz e os métodos de atualização de Forrest e Tomlin (1972) e Reid (1982), que foram implementados e desenvolvidos também por Silva (2002), como parte desta pesquisa.

Os testes foram realizados sobre um conjunto de exemplares gerados aleatoriamente, os quais tinha a estrutura escada seguinte: 


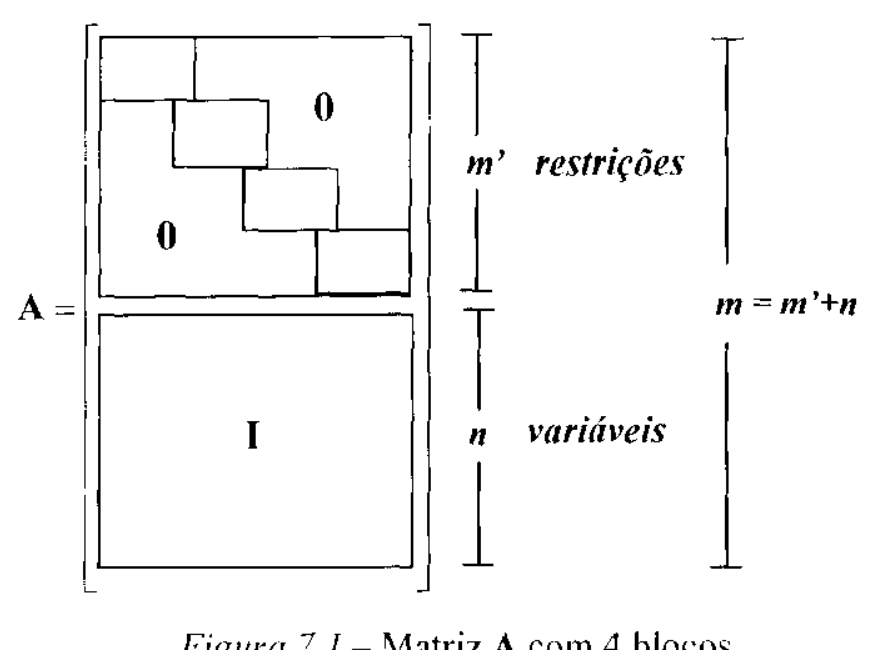

Assim, $m^{\prime}$ ou $(m-n)$ define o número de restrições da matriz $\boldsymbol{A}$ e $n$ o número de variáveis, para o problema canalizado na forma (3.1).

Para gerar um problema cuja matriz dos coeficientes tem a forma representada acima, o seguintes 4 parâmetros devem ser informados: a quantidade de blocos, o número de linhas, número de colunas de cada bloco e número de colunas de interseção.

Os testes realizados nesta seção foram feitos usando os problemas gerados aleatoriamente de tamanhos pequeno e médio, o número de linhas $\left(m^{\prime}\right)$ e colunas $(n)$ variam entre 50 e 3000 aproximadamente e a densidade (\%) varia entre $0,2 \%$ e $6 \%$ aproximadamente. Para cada conjunto de parâmetro fixado, foram resolvidos 10 exemplares e calculada a média dos resultados obtidos.

Para todos os resultados reportados nesta seção, os testes foram realizados $\mathrm{cm}$ um computador AMD Athon 1800:- (1500 MHz) com 1GB de RAM. A linguagem C foi utilizada nas implementações.

Considere que a decomposição (ou fatoração) refere-se a decompor a matriz base em $\boldsymbol{L} \boldsymbol{U}$ e a atualização, uma vez identificada as colunas que entra e sai da base, é a maneira de atualizar a matriz triangular superior $\boldsymbol{U}$ em função da anterior.

$\Lambda$ scguir, são apresentados nas Tabelas de 7.1 a 7.4 os resultados dos experimentos, variandose a freqüencia de refatoração (isto é, número de iterações feitas antes de uma nova decomposiçâo LU da matriz básica: $100, \sqrt{m^{\prime}}, 3 \sqrt{m^{\prime}} / 2$ e $\left.0.1 m^{\prime}\right)$. Os itens a serem analisados são: número de iterações realizadas, tempo total de resolução, preenchimento (inserção de novos elementos não nulos feito por atualização, tempo gasto por atualização, preenchimento por decomposição e tempo gasto por decomposição.

Com relação ao número de iterações realizadas, foi observado que não houve diferença significante para todos os parâmetros, mas, o valor 100 para a refatoração da base (isto é, a cada 
100 iterações a decomposição LU é feita) foi ligeiramente melhor para a maioria dos problemas (Silva, 2002).

$\Lambda$ Tabela 7.1 apresenta o tempo total de resolução variando-se a frequêencia de refatoração.

Tabela 7.1 - Comparação entre o tempo de resolução

\begin{tabular}{|c|c|c|c|c|c|}
\hline \multicolumn{6}{|c|}{ tempo de resolução em segundos } \\
\hline \multirow[b]{2}{*}{$m^{\prime} \times n$} & \multirow[b]{2}{*}{$\%$} & \multicolumn{4}{|c|}{ freqüência de refatoração $L U$} \\
\hline & & 100 & $\sqrt{m^{\prime}}$ & $1.5^{\sqrt{m^{\prime}}}$ & $0.1 m^{\prime}$ \\
\hline $50 \times 401$ & 4.24 & 0.17 & 0.27 & 0.23 & 0.32 \\
\hline $150 \times 401$ & 2.24 & 0.86 & 0.82 & 0.75 & 0.79 \\
\hline $200 \times 401$ & 1.25 & 0.81 & 0.64 & 0.60 & 0.59 \\
\hline $100 \times 1001$ & 2.10 & 3.29 & 7.35 & 5.86 & $7 . \overline{35}$ \\
\hline $250 \times 1001$ & 0.90 & 8.04 & 11.89 & 9.78 & 9.57 \\
\hline $500 \times 1001$ & 0.50 & 12.32 & 17.15 & 14.63 & 12.84 \\
\hline $500 \times 2001$ & 0.45 & 137.08 & 187.62 & 162.40 & 156.18 \\
\hline $1000 \times 2001$ & 0.25 & 240.34 & 290.70 & 266.72 & 240.34 \\
\hline $750 \times 3001$ & 0.43 & 938.18 & 1117.60 & 1028.1 & 955.76 \\
\hline $400 \times 51$ & 5.88 & 0.02 & 0.02 & 0.01 & 0.02 \\
\hline $400 \times 151$ & 1.49 & 0.21 & 0.15 & 0.15 & 0.14 \\
\hline $400 \times 201$ & 2.97 & 0.32 & 0.20 & 0.20 & 0.21 \\
\hline $1000 \times 101$ & 2.65 & 0.10 & 0.07 & 0.07 & 0.10 \\
\hline $1000 \times 251$ & 0.60 & 0.89 & 0.63 & 0.68 & 0.89 \\
\hline $1000 \times 501$ & 0.60 & 3.89 & 3.43 & 3.44 & 3.89 \\
\hline $2000 \times 501$ & 0.30 & 5.80 & 5.19 & 5.41 & 6.33 \\
\hline $2000 \times 1001$ & 0.53 & 37.29 & 39.99 & 37.58 & 40.07 \\
\hline $3000 \times 751$ & 1.00 & 26.75 & 27.50 & 26.64 & 28.46 \\
\hline
\end{tabular}

Pela Tabela 7.1, o tempo de resolução para o valor 100 foi menor na maioria dos problemas quando $n>m^{\prime}$ e o maior quando $m^{\prime}>n$. Para o valor $\sqrt{m^{\prime}}$ ocorreu cxatamente o oposto, ou seja, $\sqrt{m^{\prime}}$ foi o melhor quando $m^{\prime}>n$ e pior quando $n>m^{\prime}$. Para os valores $1.5 \sqrt{m^{\prime}}$ e $0.1 m^{\prime}$ os resultados foram próximos em quase todos os problemas quando $n>m^{\prime}$ enquanto que para $m^{\prime}>n$, os resultados com $\sqrt{m^{\prime}}$ e $1.5 \sqrt{m^{\prime}}$ foram próximos.

A Tabela 7.2 a seguir traz um resumo do desempenho do método, variando-se a freqüência de refatoração, sendo do melhor para o pior desempenho percorrendo as colunas da esquerda para a direita, ou scja, na primeira coluna estão os valores que apresentaram melhor desempenho, depois o valor que apresentou o segundo melhor desempenho e assim sucessivamente. 
Tabela 7.2 - Comparação entre alguns valores para o número de atualizaçōes

\begin{tabular}{|c|c|c|c|c|}
\hline \multicolumn{5}{|c|}{ Classificaçāo dos valores para o número de atualizaçóes } \\
\hline & \multicolumn{4}{|c|}{$n>m^{\prime}$} \\
\hline Número de iterações & 100 & $\sqrt{m^{\prime}}$ & $0.1 m^{\prime}$ & $1.5 \sqrt{m^{\prime}}$ \\
\hline Tempo de resolução & 100 & $0.1 m^{\prime}$ & $1.5 \sqrt{m^{\prime}}$ & $\sqrt{m^{\prime}}$ \\
\hline Preenchimento/atualização & $\sqrt{m^{4}}$ & $1.5 \sqrt{m}$ & 100 & $0.1 m^{\prime}$ \\
\hline Tempo/atualização & 100 & $1.5 \sqrt{m^{\prime}}$ & $0.1 m^{\prime}$ & $\sqrt{m^{\prime}}$ \\
\hline Preenchimento/decomposiçāo & 100 & $\sqrt{m^{\prime}}$ & $1.5 \sqrt{m^{\prime}}$ & $0.1 \mathrm{~m}$ \\
\hline \multirow[t]{2}{*}{ Tempo/decomposiçāo } & 100 & $1.5 \sqrt{m^{\prime}}$ & $0.1 m^{\prime}$ & $\sqrt{m^{\prime}}$ \\
\hline & \multicolumn{4}{|c|}{$m^{\prime}>n$} \\
\hline Número de iteraçōes & 100 & $0.1 m^{\prime}$ & $\sqrt{m}$ & $1.5 \sqrt{m}$ \\
\hline Tempo de resolução & $\sqrt{m^{\prime}}$ & $1.5 \sqrt{m^{\prime}}$ & $0.1 \mathrm{~m}^{\prime}$ & 100 \\
\hline Preenchimento/atualização & 100 & $1.5 \sqrt{m^{\prime}}$ & $\sqrt{m^{\prime}}$ & $0.1 \mathrm{~m}$ \\
\hline Tempo/atualização & $100,1.5 \sqrt{m^{\prime}}, \sqrt{m^{\prime}}$ e $0.1 m$ & & & \\
\hline Preenchimento/decomposição & 100 & $1.5 \sqrt{m^{\prime}}$ & $\sqrt{m^{\prime}}$ & $0.1 \mathrm{~m}^{\prime}$ \\
\hline Tempo/decomposição & 100 & $1.5 \sqrt{m^{\prime}}$ & $\sqrt{m^{\prime}}$ & $0.1 \mathrm{~m}^{\prime}$ \\
\hline
\end{tabular}

Obscrvando a Tabela 7.2 vemos que o valor 100 foi o melhor em quase todos itens analisados para a maioria dos problemas $\left(n>m^{\prime} \mathrm{e} m^{\prime}>n\right)$.

Com relação ao número de iterações, o melhor valor foi 100 para todos os problemas e o pior foi $3 \sqrt{m^{\prime}} / 2$. Para o tempo total de resolução, o valor 100 foi melhor quando $n>m$ ' e o valor $\sqrt{m^{\prime}}$ quando $m^{\prime}>n$. Em se tratando do preenchimento por atualização o valor $\sqrt{m^{\prime}}$ foi o melhor quando $n>m^{\prime}$ e o valor 100 foi melhor para $m^{\prime}>n$

O preenchimento por decomposição foi menor para o valor 100 nos casos em que $m$ ' $>n$ e $n>m$ ' e maior em todos os problemas para o valor $0.1 m^{\prime}$. O tempo por atualização foi menor para o valor 100 quando $n>m$ ' e igual para todos os valores quando $m$ ' $>n$. Finalmente, o tempo por decomposição foi menor para o valor 100 tanto no caso em que $n>m$ ' quanto no caso $\mathrm{cm}$ que $m^{\prime}>n$.

Foram feitos outros testes para valores menores que $\sqrt{m^{\prime}}$ e maiores que $0.1 m^{\prime}$ e os resultados obtidos foram piores que estes apresentados em todos os itens analisados. Por ter apresentado um melhor desempenho na maioria dos itens o valor 100 foi utilizado para os demais testes.

Para os exemplos que tratamos, fizemos algumas combinações das heurísticas de Grau Mínimo e Markowitz com os métodos de atualização de Forrest e Tomlin (1972) e Reid (1982). Para csses métodos de atualização de Forrest e Tomlin (1972) e Reid (1982), a Tabcla 7.3 apresenta os resultados para o tempo total de resolução combinando as heuristicas e as variantes. 
Tabela 7.3 - Tempo total de resolução de problemas usando gerador escada

\begin{tabular}{|c|c|c|c|c|c|c|}
\hline \multicolumn{7}{|c|}{ tempo de resolução em segundos } \\
\hline \multirow[b]{2}{*}{$m \times n$} & \multirow[b]{2}{*}{ iterações } & \multirow[b]{2}{*}{$\%$} & \multicolumn{2}{|c|}{ Grau Mínimo } & \multicolumn{2}{|c|}{ Markowitz } \\
\hline & & & $F \& T$ & Reid & $F \& T$ & Reid \\
\hline $50 \times 401$ & 70.5 & 4.24 & 0.51 & 0.17 & 0.51 & 0.17 \\
\hline $150 \times 401$ & 180.1 & 2.24 & 1.55 & 0.86 & 1.76 & 1.02 \\
\hline $200 \times 401$ & 211.3 & 1.25 & 1.53 & 0.81 & 1.69 & 0.85 \\
\hline $100 \times 1001$ & 148.2 & 2.10 & 7.18 & 3.29 & 10.05 & 11.87 \\
\hline $250 \times 1001$ & 307.6 & 0.90 & 14.02 & 8.04 & 20.53 & 16.26 \\
\hline $500 \times 1001$ & 519.4 & 0.50 & 23.05 & 12.32 & 32.24 & 21.18 \\
\hline $500 \times 2001$ & 617 & 0.45 & 318.0 & 137.08 & 537.56 & 243.30 \\
\hline $1000 \times 2001$ & 1050.6 & 0.25 & 544.2 & 240.34 & 950.40 & 354.38 \\
\hline $750 \times 3001$ & 1008 & 0.43 & 1826.2 & 938.18 & 2746.2 & 1877.20 \\
\hline $400 \times 51$ & 94.3 & 5.88 & 0.02 & 0.02 & 0.02 & 0.02 \\
\hline $400 \times 151$ & 222.8 & 1.49 & 0.36 & 0.21 & 0.39 & 0.22 \\
\hline $400 \times 201$ & 253 & 2.97 & 0.54 & 0.32 & 0.59 & 0.33 \\
\hline $1000 \times 101$ & 196 & 2.65 & 0.17 & 0.10 & 0.17 & 0.09 \\
\hline $1000 \times 251$ & 401.5 & 0.60 & 1.45 & 0.89 & 1.59 & 0.94 \\
\hline $1000 \times 501$ & 618.5 & 0.60 & 7.20 & 3.89 & 8.83 & 4.49 \\
\hline $2000 \times 501$ & 797.6 & 0.30 & 9.60 & 5.80 & 11.44 & 6.63 \\
\hline $2000 \times 1001$ & 1248.8 & 0.53 & 58.85 & 37.29 & 79.96 & 55.45 \\
\hline $3000 \times 751$ & 1245.8 & 1.00 & 40.16 & 26.75 & 52.02 & 34.36 \\
\hline
\end{tabular}

De acordo com a Tabela 7.3, para a maioria dos problemas $\left(n>m^{\prime}\right.$ e $\left.m^{\prime}>n\right)$ o tempo total de resolução das combinações Grau Mínimo e Reid e Markowitz e Reid apresentaram melhor desempenho, sendo que a primeira combinação foi a melhor entrc as duas, assim como para o tempo por atualização (Silva, 2002).

A Tabela 7.4 apresenta o tempo de decomposição com as combinações das heurísticas. 
Tabela 7.4 - Tempo por decomposição

\begin{tabular}{|c|c|c|c|c|c|c|}
\hline \multicolumn{7}{|c|}{ tempo por decomposição (segundos) } \\
\hline \multirow[b]{2}{*}{ problemas } & \multirow[b]{2}{*}{ iterações } & \multirow[b]{2}{*}{$\%$} & \multicolumn{2}{|c|}{ Grau Mínimo } & \multicolumn{2}{|c|}{ Markowitz } \\
\hline & & & F\&T & Reid & $F \& T$ & Reid \\
\hline $50 \times 401$ & 70.5 & 4.24 & 0.00 & 0 & 0.00 & 0 \\
\hline $150 \times 401$ & 180.1 & 2.24 & 0.01 & 0.01 & 0.02 & 0.02 \\
\hline $200 \times 401$ & 211.3 & 1.25 & 0.01 & 0.01 & 0.02 & 0.02 \\
\hline $100 \times 1001$ & 148.2 & 2.10 & 0.25 & 0.21 & 0.38 & 0.33 \\
\hline $250 \times 1001$ & 307.6 & 0.90 & 0.34 & 0.31 & 0.50 & 0.44 \\
\hline $500 \times 1001$ & 519.4 & 0.50 & 0.38 & 0.31 & 0.51 & 0.45 \\
\hline $500 \times 2001$ & 617 & 0.45 & 2.17 & 2.04 & 2.87 & 2.71 \\
\hline $1000 \times 2001$ & 1050.6 & 0.25 & 2.27 & 2.23 & 3.03 & 2.83 \\
\hline $750 \times 3001$ & 1008 & 0.43 & 6.78 & 6.60 & 9.63 & $9 . \overline{18}$ \\
\hline $400 \times 51$ & 94.3 & 5.88 & 0.00 & 0.00 & 0.00 & 0.00 \\
\hline $400 \times 151$ & 222.8 & 1.49 & 0.00 & 0.00 & 0.00 & 0.00 \\
\hline $400 \times 201$ & 253 & 2.97 & 0.00 & 0.00 & 0.00 & 0.01 \\
\hline $1000 \times 101$ & 196 & 2.65 & 0.00 & 0.00 & 0.00 & 0.00 \\
\hline $1000 \times 251$ & 401.5 & 0.6 & 0.00 & 0.00 & 0.01 & 0.01 \\
\hline $1000 \times 501$ & 618.5 & 0.6 & 0.03 & 0.03 & 0.06 & 0.05 \\
\hline $2000 \times 501$ & 797.6 & 0.3 & 0.03 & 0.03 & 0.08 & 0.08 \\
\hline $2000 \times 1001$ & 1248.8 & 0.53 & 0.44 & 0.38 & 0.70 & 0.62 \\
\hline $3000 \times 751$ & 1245.8 & 1.0 & 0.26 & 0.21 & $0 . \overline{56}$ & 0.51 \\
\hline
\end{tabular}

Com relação ao tempo por decomposição, de acordo com a Tabela 7.5 , houve uma troca nos pares de combinações na maioria dos problemas considerando os casos em que $n>m$ e $m>n$, pois as combinações Grau Mínimo com F\&T 1 e Grau Mínimo com Reid apresentaram resultados próximos e melhores que as combinações Markowitz com F\&T e Markowitz com Reid. As difcrenças no tempo gasto por decomposição entre todas combinações, nos casos onde $n>m$ e $m>$ $n$, foram menores para os problemas pequenos.

Silva (2002) também observou que independentemente do método de atualização, a heurística de Markowit\% apresentou menos preenchimento por atualização. A combinação que menos introduz preenchimentos foi de Markowitz com Forrest \& Tomlin.

Em geral, tcmos que a melhor combinação foi a heurística de Grau Mínimo com Reid, e a pior combinação foi com a heurística de Markowitz com Forrest \& Tomlin. Para mais detalhes desses resultados veja Silva (2002). Os resultados a seguir utilizam a combinação da heurística Markowity com Reid.

Comparamos agora o método DSBU com o pacote CPLEX $7.5 \mathrm{com}$ relação ao número de itcrações e tempo de resolução, utilizando os mesmos problemas e as melhores combinações cntre heurísticas e variantes para cada gerador, identificadas anteriormente. O CPI.EX foi utilizado sem ativar o pré-processador (veja apêndice A), foi usado também o método dual simplex (tranopt) para 
resolver os problemas e a freqüência de refatoração foi 100 (mas, quando não é informado o intervalo, o pacote CPLEX determina dinamicamente a frequiência da refatoração da base, com o objetivo de minimizar o tempo por iteração), o mesmo usado pelo DSBU.

A Tabela 7.5 apresenta os resultados, em que $n b$ representa o númcro de blocos, $m 1$ e $n 1$ representam os números de linhas e colunas, respectivamente em cada bloco. O número de colunas em comum a cada dois blocos consecutivos fixado $\mathrm{em} 1$.

Tabela 7.5 - Comparação entre DSBU e CPLEXX

\begin{tabular}{|c|c|c|c|c|c|c|c|c|}
\hline \multicolumn{5}{|c|}{ problemas } & \multicolumn{2}{|c|}{ iteraçōes } & \multicolumn{2}{|c|}{ tempo } \\
\hline$n b$ & $m 1$ & $n 1$ & $\%$ & $m^{\prime} \times n$ & CPLEX & $D S B U$ & CPLEX & $D S B U$ \\
\hline 20 & 2 & 17 & 4.24 & $50 \times 401$ & 70.4 & 70.5 & 0 & 0.17 \\
\hline 50 & 3 & 9 & 2.24 & $150 \times 401$ & 192.3 & 180.1 & 0.02 & 0.86 \\
\hline 100 & 2 & 5 & 1.25 & $200 \times 401$ & 221.3 & 211.3 & 0.02 & 0.81 \\
\hline 50 & 2 & 21 & 2.10 & $100 \times 1001$ & 147.1 & 148.2 & 0.02 & 3.29 \\
\hline 125 & 2 & 9 & 0.90 & $250 \times 1001$ & 309.4 & 307.6 & 0.03 & 8.04 \\
\hline 250 & 2 & 5 & 0.50 & $500 \times 1001$ & 540.7 & 519.4 & 0.06 & 12.32 \\
\hline 250 & 2 & 9 & 0.45 & $500 \times 2001$ & 631.6 & 617.2 & 0.09 & 137.08 \\
\hline 500 & 2 & 5 & 0.25 & $1000 \times 2001$ & 1084.5 & 1050.6 & 0.25 & 240.34 \\
\hline 250 & 3 & 9 & 0.43 & $750 \times 3001$ & 1047.3 & 1008.1 & 0.32 & 938.18 \\
\hline 25 & 16 & 3 & 5.88 & $400 \times 51$ & 97.6 & 94.3 & 0.03 & 0.02 \\
\hline 50 & 8 & 4 & 1.49 & $400 \times 151$ & 261.9 & 222.8 & 0.04 & 0.21 \\
\hline 100 & 4 & 3 & 2.97 & $400 \times 201$ & 273.1 & 253.4 & 0.04 & 0.32 \\
\hline 50 & 20 & 3 & 2.65 & $1000 \times 101$ & 231.4 & 196.7 & 0.07 & 0.10 \\
\hline 125 & 8 & 3 & 0.60 & $1000 \times 251$ & 430.9 & 401.5 & 0.13 & 0.89 \\
\hline 250 & 4 & 3 & 0.60 & $1000 \times 501$ & 859.2 & 618.5 & 0.16 & 3.89 \\
\hline 250 & 8 & 3 & 0.30 & $2000 \times 501$ & 842.8 & 797.6 & 0.46 & 5.80 \\
\hline 500 & 4 & 3 & 0.53 & $2000 \times 1001$ & 1333.4 & 1248.8 & 0.65 & 37.29 \\
\hline 250 & 12 & 4 & 1.00 & $3000 \times 751$ & 1407.2 & 1245.8 & 1.35 & 26.75 \\
\hline
\end{tabular}

Analisando a Tabela 7.5, vemos que na maioria dos problemas o DSBU realizou um número menor de iteraçōes. $\Lambda$ superioridade do método DSBU foi maior nos problemas com $m^{\prime}>n$. O tempo de resolução do CPLEX foi muito inferior ao tempo do DSBU, principalmente quando $n>m^{\prime}$. Os problemas com $m^{\prime}>n$ exigiram um número maior de iterações do que com $n>m$, porém o tempo total de resolução foi menor.

\subsection{Extensões na Busca Unidimensional}

Daremos nesta seção, os resultados computacionais obtidos para o método DSBU com as extensões propostas na busca unidimensional descritas no capítulo 3 e para o método dual simplex padrão, em que são feitas algumas comparações para três tipos de estrutura na matriz $\mathbf{A}$. 
Para cstes resultados o método DSBU foi implementado utilizando a forma produto da inversa. Para cada tamanho (dimensão) do problema, que define um exemplar com $m$ ' restriçôes e $n$ variáveis (veja Figura 7.1), foram rodados 20 exemplos com os dados gerados aleatoriamente. Os testes desta seção em diante, foram realizados em uma máquina de 2.80 (jHz com 512 Mbytes de RAM em ambiente Windows 2000 .

O tempo (em segundos) e o número de iterações, reportados a seguir, são as médias dos 20 exemplos para cada tamanho do problema. Para a apresentação dos resultados, considere:

- BC_1: método dual simplex com busca exata (completa) com ordenação preliminar:

- BC_2: método dual simplex com busca exata com ordenação $\mathrm{cm}$ processo;

- SB: método dual simplex padrão (usual) sem busca;

- BP: mćtodo dual simplex com busca inexata (parcial);

- AR: método dual simplex com a regra de Armijo (busca inexata).

Para a regra de Armijo, utilizamos $\varepsilon=0.2$ (veja capítulo 3, um valor usual, segundo Luenbeger, 1984). Testamos outros valores, mas o desempenho foi pior.

Lembre-se que os valores de $\delta$ devem ser ordenados em ordem crescente $\mathrm{e}$ indicam os pontos de não-diferenciabilidade de $h(\hat{\hat{\lambda}}+\delta \boldsymbol{\eta})$. A primeira questão que surge, é sobre a grandeza de $r$ (comparada com $n$, veja capítulo 3), pois isto pode levar a ordenações de vetores de dimensões elevadas para problemas de grande porte: Como esta grandeza $r$ é influenciada pela esparsidade da matriz de restrições? Uma outra questão é sobre a grandeza de $\ell$ (comparada com $r$ ): Será necessário ordenar todo o vetor de pontos de não diferenciação? E. por último, compensa a busca unidimensional exata? Isto é, após um avanço na direção dual simplex, não seria melhor mudar de direção? Para responder estas questões, apresentamos os resultados.

\subsubsection{Problemas Densos}

Nesta seção apresentamos os resultados obtidos para problemas com praticamente $100 \%$ dos elementos das $(m-n)$ restrições diferentes de zero. A Tabela 7.6 apresenta os resultados para o método dual simplex linear com busca completa $\left(\mathrm{BC}_{-} 1\right) \mathrm{e}$ método dual simplex padrão (SB). 


\begin{tabular}{|c|c|c|c|c|c|}
\hline \multirow[b]{2}{*}{ exemplar } & \multirow[b]{2}{*}{$m^{\prime} \times n$} & \multicolumn{2}{|c|}{ iteraçōes } & \multicolumn{2}{|c|}{ tempo } \\
\hline & & $B C \_1$ & $S B$ & $B C_{1} 1$ & $S B$ \\
\hline 1 & $100 \times 100$ & 133.2 & 419.3 & 0.06 & 0.11 \\
\hline 2 & $200 \times 100$ & 205.0 & 653.7 & 0.08 & 0.24 \\
\hline 3 & $300 \times 100$ & 235.5 & 785.8 & 0.11 & 0.42 \\
\hline 4 & $200 \times 200$ & 301.3 & 914.7 & 0.38 & 1.83 \\
\hline 5 & $300 \times 200$ & 389.6 & 1200.6 & 0.60 & 2.43 \\
\hline 6 & $20 \times 400$ & 63.5 & 432.7 & 0.23 & 1.31 \\
\hline 7 & $100 \times 200$ & 175.0 & 642.7 & 0.19 & 0.66 \\
\hline 8 & $100 \times 400$ & 241.1 & 1150.3 & 1.04 & 4.61 \\
\hline 9 & $200 \times 400$ & 401.2 & 1618.1 & 1.98 & 8.56 \\
\hline 10 & $400 \times 200$ & 475.2 & 1481.4 & 0.94 & 3.20 \\
\hline 11 & $400 \times 400$ & 622.8 & 1980.1 & 3.21 & 11.27 \\
\hline & Média & 294.8 & 1025.4 & 0.86 & 3.18 \\
\hline
\end{tabular}

A Tabela 7.6, mostra que a busca unidimensional completa faz reduzir consideravelmente o número de iterações com relação ao método dual simplex usual, executando aproximadamente, $\mathrm{cm}$ média, apenas $28 \%$ das iterações de SB. Observe que o tempo por iteração, cm média, foi praticamente o mesmo para os dois métodos.

No excmplar 6, em que o número de variáveis é muito maior do que o número de restrições, obscrvamos a maior diferença no número de iterações. Portanto, testamos mais 4 exemplares com esta característica. Os resultados estão na Tabela 7.7 .

\begin{tabular}{|c|c|c|c|c|c|}
\hline \multirow[b]{2}{*}{ exemplar } & \multirow[b]{2}{*}{$m \times n$} & \multicolumn{2}{|c|}{ iteraçôes } & \multicolumn{2}{|c|}{ tempo } \\
\hline & & $B C 1$ & $S B$ & $B C \_1$ & $S B$ \\
\hline 12 & $10 \times 400$ & 35.2 & 300.8 & 0.12 & 0.83 \\
\hline 6 & $20 \times 400$ & 63.5 & 432.7 & 0.24 & 1.39 \\
\hline 13 & $30 \times 400$ & 89.3 & 567.1 & 0.32 & 1.82 \\
\hline 14 & $40 \times 400$ & 109.1 & 661.8 & 0.45 & 2.17 \\
\hline 15 & $50 \times 400$ & 132.0 & 790.8 & 0.54 & 2.93 \\
\hline & Média & 85.8 & 550.6 & 0.33 & 1.82 \\
\hline
\end{tabular}

Notamos da Tabela 7.7, que a maior diferença foi para o exemplar 12, com o número de variáveis é 40 vezes o número de restrições. Fista diferença no número de iteraçôes diminui à medida que o número de restrições aumenta.

Apresentamos agora os resultados para o método de busca unidimensional inexata (regra de Armijo - AR) descrito no capítulo 3, comparando com BC_1. $\Lambda$ Tabela 7.8 exibe os resultados obtidos. 


\begin{tabular}{|c|c|c|c|c|c|}
\hline \multirow[b]{2}{*}{ exemplar } & \multirow[b]{2}{*}{$m^{\prime} \times n$} & \multicolumn{2}{|c|}{ iteraçōes } & \multicolumn{2}{|c|}{ tempo } \\
\hline & & $B C \_1$ & $A R$ & $B C 1$ & $A R$ \\
\hline 1 & $100 \times 100$ & 133.2 & 171.2 & 0.06 & 0.06 \\
\hline 2 & $200 \times 100$ & 205.0 & 285.3 & 0.08 & 0.11 \\
\hline 3 & $300 \times 100$ & 235.5 & 341.2 & 0.11 & 0.18 \\
\hline 4 & $200 \times 200$ & 301.3 & 494.9 & 0.38 & 0.70 \\
\hline 5 & $300 \times 200$ & 389.6 & 751.4 & 0.60 & 1.18 \\
\hline 6 & $20 \times 400$ & 63.5 & 107.6 & 0.23 & 0.39 \\
\hline 7 & $100 \times 200$ & 175.0 & 225.8 & 0.19 & 0.24 \\
\hline 8 & $100 \times 400$ & 241.1 & 342.3 & 1.04 & 1.42 \\
\hline 9 & $200 \times 400$ & 401.2 & 562.0 & 1.98 & 2.78 \\
\hline 10 & $400 \times 200$ & 475.2 & 924.5 & 0.94 & 1.72 \\
\hline \multirow[t]{2}{*}{11} & $400 \times 400$ & 622.8 & 1351.6 & 3.21 & 6.9 \\
\hline & Média & 294.8 & 505.2 & 0.86 & 1.42 \\
\hline
\end{tabular}

A clássica regra de Armijo foi muito inferior do que a busca completa, fazendo em média $70 \%$ mais iteraçōes e gastando aproximadamente $53 \%$ mais tempo. No entanto, a regra de Armijo ć melhor do que o método dual simplex padrão (veja Tabela 7.6), a qual reduz em média o númcro de iterações pela metade.

No método dual simplex com busca unidimensional, uma vez calculados os $\delta$ s. estes precisam ser ordenados. Uma questão que surge é se este procedimento de ordenação pode consumir muito tempo? Assim, relatamos o tempo de ordenação para o método BC_1, apresentados na Tabela 7.9, em que TT indica o tempo de resolução e TO o tempo de ordenação (método bolha) para todas as iterações. Fizemos os testes também com o método heapsort, para o ordenar o vetor de $\delta^{\prime}$ s, e a melhora no tempo não foi significante.

Tabela 7.9- Tempo Total $\times$ Tempo de Ordenação
\begin{tabular}{|c|c|c|c|}
\hline & & \multicolumn{2}{|c|}{ tempo } \\
\hline exemplar & $\boldsymbol{m}^{\prime} \times \boldsymbol{n}$ & $T T$ & TO \\
\hline 1 & $\mathbf{1 0 0 \times 1 0 0}$ & 0.06 & 0.00 \\
\hline 2 & $\mathbf{2 0 0 \times 1 0 0}$ & 0.08 & 0.00 \\
\hline 3 & $\mathbf{3 0 0 \times 1 0 0}$ & 0.11 & 0.01 \\
\hline 4 & $\mathbf{2 0 0 \times 2 0 0}$ & 0.38 & 0.03 \\
\hline 5 & $\mathbf{3 0 0 \times 2 0 0}$ & 0.60 & 0.05 \\
\hline 6 & $\mathbf{2 0 \times 4 0 0}$ & 0.23 & 0.03 \\
\hline 7 & $\mathbf{1 0 0 \times 2 0 0}$ & 0.19 & 0.02 \\
\hline 8 & $\mathbf{1 0 0 \times 4 0 0}$ & 1.04 & 0.08 \\
\hline 9 & $\mathbf{2 0 0 \times 4 0 0}$ & 1.98 & 0.07 \\
\hline 10 & $\mathbf{4 0 0 \times 2 0 0}$ & 0.94 & 0.04 \\
\hline 11 & $\mathbf{4 0 0 \times \mathbf { 4 0 0 }}$ & 3.21 & 0.10 \\
\hline & Média & $\mathbf{0 . 8 6}$ & $\mathbf{0 . 0 3}$ \\
\hline
\end{tabular}


Podemos observar que o custo de ordenação não é muito elevado, pois em média, corresponde a menos de $5 \%$ do tempo total. Apenas para os exemplares 6,7 e 8 esta porcentagem foi de quase $10 \%$, casos em que os números de variáveis são maiores do que os númcros de restrições. Reportamos agora o número de deltas $(r)$ em função de $n$ para as primeiras iterações (cerca de $10 \%$ do total de iterações) e o valor de $\ell r$ para as duas primeiras iteraçôes, já que para as outras, os valores diminuem suavemente. Na Tabela 7.10 são apresentados os resultados.

\begin{tabular}{|c|c|c|c|c|}
\hline \multirow[b]{2}{*}{ exemplar } & \multirow[b]{2}{*}{$m^{\prime} \times n$} & \multirow[b]{2}{*}{$r / n$} & \multicolumn{2}{|c|}{ elr iteração } \\
\hline & & & $1^{a}$ & $2^{a}$ \\
\hline 1 & $100 \times 100$ & 0.86 & 0.36 & 0.18 \\
\hline 2 & $200 \times 100$ & 0.86 & 0.36 & 0.18 \\
\hline 3 & $300 \times 100$ & 0.86 & 0.36 & 0.18 \\
\hline 4 & $200 \times 200$ & 0.86 & 0.36 & 0.18 \\
\hline 5 & $300 \times 200$ & 0.87 & 0.33 & 0.18 \\
\hline 6 & $20 \times 400$ & 0.81 & 0.35 & 0.18 \\
\hline 7 & $100 \times 200$ & 0.88 & 0.34 & 0.17 \\
\hline 8 & $100 \times 400$ & 0.84 & 0.33 & 0.17 \\
\hline 9 & $200 \times 400$ & 0.84 & 0.36 & 0.17 \\
\hline 10 & $400 \times 200$ & 0.87 & 0.59 & 0.58 \\
\hline \multirow[t]{2}{*}{11} & $400 \times 400$ & 0.86 & 0.56 & 0.59 \\
\hline & Média & 0.85 & 0.39 & 0.25 \\
\hline
\end{tabular}

Da Tabela $7.10, r \approx 0.85 n$. $\Lambda$ pós as primeiras iterações, notamos que este valor tende a $0.5 n$ independente do número de variáveis. Com relação à razão $\ell r$, a média foi a mesma para a primeira e a segunda iteração nos exemplares 1-9 e nos exemplares 10 e 11 os valores foram bem diferentes. Ou seja, para estes problemas (densos) o número de deltas é muito alto, sendo em média $85 \%$ do número de variáveis e que para determinar o índice $\ell$ (busca exata) são necessários apenas $39 \%$ dos $\delta$ s (total de $r$ ) e tendem a diminuir com as iterações.

Diante dos resultados apresentados nas Tabelas 7.9 e 7.10 , investigamos as modificações propostas no capitulo 3. As Tabelas 7.11 e 7.12 fornecem os resultados.

Para a busca parcial (BP), utilizamos o parâmetro $\alpha$ (veja capítulo 3) sendo 0.35 , já que pclos resultados da Tabela 7.10 a busca foi feita utilizando em média $0.39 r$ ou $0.33 n$, dos valores de $\delta \mathrm{s}$. 


\begin{tabular}{|c|c|c|c|c|c|}
\hline \multirow[b]{2}{*}{ exemplar } & \multirow[b]{2}{*}{$m^{\prime} \times n$} & \multicolumn{2}{|c|}{ iteraçōes } & \multicolumn{2}{|c|}{ tempo } \\
\hline & & $B C 1$ & $B P$ & $B C \quad 1$ & $B P$ \\
\hline 1 & $100 \times 100$ & 133.2 & 133.2 & 0.06 & 0.05 \\
\hline 2 & $200 \times 100$ & 205.0 & 205.0 & 0.08 & 0.08 \\
\hline 3 & $300 \times 100$ & 235.5 & 235.5 & 0.11 & 0.10 \\
\hline 4 & $200 \times 200$ & 301.3 & 301.3 & 0.38 & 0.36 \\
\hline 5 & $300 \times 200$ & 389.6 & 389.6 & 0.60 & 0.59 \\
\hline 6 & $20 \times 400$ & 63.5 & 63.5 & 0.23 & 0.21 \\
\hline 7 & $100 \times 200$ & 175.0 & 175.0 & 0.19 & 0.19 \\
\hline 8 & $100 \times 400$ & 241.1 & 241.1 & 1.04 & 1.03 \\
\hline 9 & $200 \times 400$ & 401.2 & 401.2 & 1.98 & 1.96 \\
\hline 10 & $400 \times 200$ & 475.2 & 475.2 & 0.94 & 0.92 \\
\hline \multirow[t]{2}{*}{11} & $400 \times 400$ & 622.8 & 630.0 & 3.21 & 4.08 \\
\hline & Média & 294.8 & 295.5 & 0.86 & 0.87 \\
\hline
\end{tabular}

Neste caso, a busca parcial (BP) não fez praticamente nenhuma diferença no tempo computacional. No exemplar 11, a busca parcial (inexata) foi pior, pois fer mais iterações.

Fizemos testes para diferentes valores de alfa e não observamos nenhuma melhora significativa.

Na tabela 7.12, reportamos os resultados para a segunda modificação (busca exata) realizada na busca unidimensional, descrita pelo segundo procedimento de busca.

Tabela 7.12-Busca Completa $1 \times$ Busca Completa 2
\begin{tabular}{|c|c|c|c|}
\hline \hline & & \multicolumn{2}{|c|}{ tempo } \\
\hline exemplar & $m^{\prime} \times n$ & $B C 1$ & $B C 2$ \\
\hline 1 & $100 \times \mathbf{1 0 0}$ & 0.06 & 0.05 \\
\hline 2 & $200 \times \mathbf{1 0 0}$ & 0.08 & 0.07 \\
\hline 3 & $300 \times 100$ & 0.11 & 0.10 \\
\hline 4 & $200 \times 200$ & 0.38 & 0.36 \\
\hline 5 & $300 \times \mathbf{2 0 0}$ & 0.60 & 0.59 \\
\hline 6 & $20 \times \mathbf{4 0 0}$ & 0.23 & 0.22 \\
\hline 7 & $\mathbf{1 0 0 \times 2 0 0}$ & 0.19 & 0.17 \\
\hline 8 & $\mathbf{1 0 0 \times 4 0 0}$ & 1.04 & 0.95 \\
\hline 9 & $\mathbf{2 0 0 \times 4 0 0}$ & 1.98 & 1.80 \\
\hline 10 & $\mathbf{4 0 0 \times 2 0 0}$ & 0.94 & 0.91 \\
\hline 11 & $\mathbf{4 0 0 \times 4 0 0}$ & 3.21 & 3.24 \\
\hline & Média & $\mathbf{0 . 8 6}$ & $\mathbf{0 . 7 6}$ \\
\hline
\end{tabular}

Da tabela 7.12, observamos para estes problemas densos, uma suave melhora no tempo computacional, para $\mathrm{BC}_{-} 2 \mathrm{~cm}$ relação a $\mathrm{BC}_{-}$, em que conseguimos uma redução no tempo (em média) em torno de $6 \%$. Note que não apresentamos o número de iterações para $B C_{-} 1$, que é o mesmo para $\mathrm{BC}_{-} 2$, pois ambas são buscas exatas. 
Lembre-se que o que muda da busca completa 1 para a busca completa 2 , é o método de ordenação. No $2^{\circ}$ caso é o método 'bolha' que parcce ser melhor, pois $\ell$ é bem menor que $r$ c não precisa ordenar todo o vetor, já que a ordenação pode ser interrompida assim que $\ell$ é determinado.

A seguir, a Figura 7.2 apresenta o desempenho computacional (tempo) dos quatro procedimentos de busca $\mathrm{c}$ do método dual simplex padrão, para os 11 exemplares de problemas densos que trabalhamos.

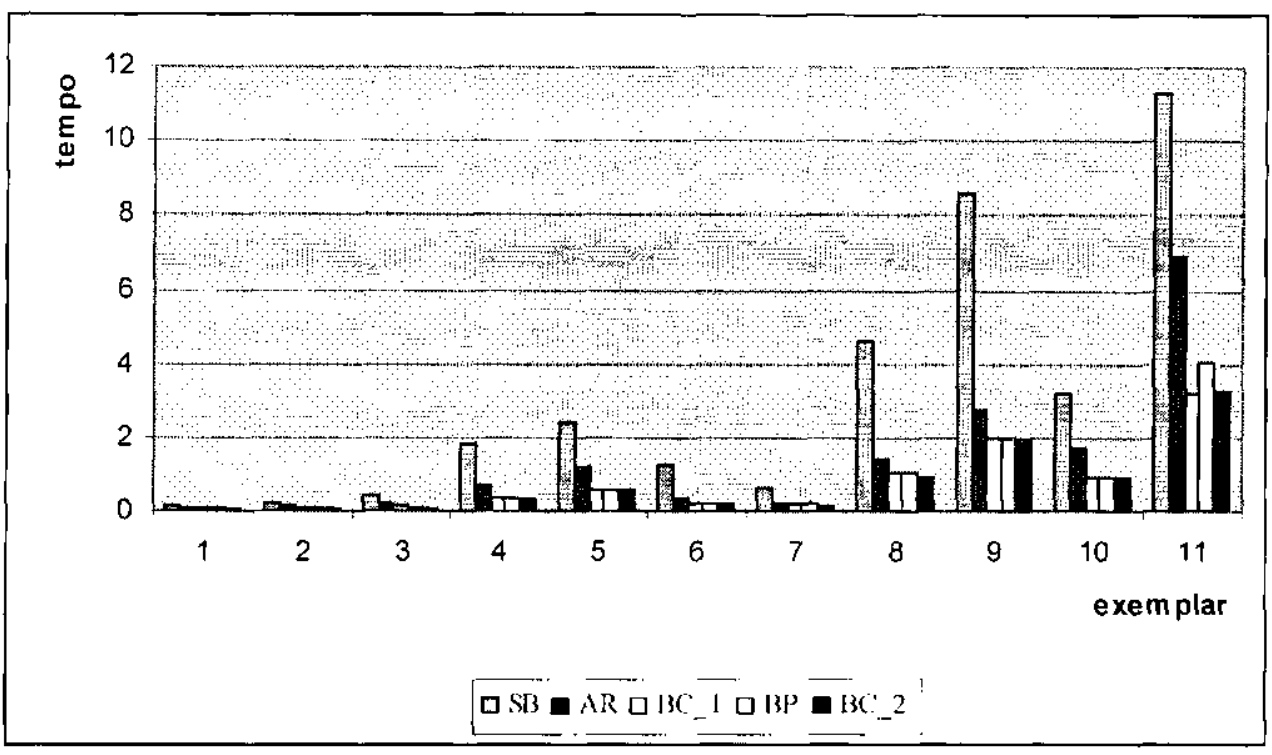

Figura 7,2 - Tempo de Resolução - Problemas Densos

Da Figura 7.2, podemos dizer que de um modo geral, a busca completa com ordenação em processo ( $\mathrm{BC}_{-}$2) foi um pouco mclhor do que a busca parcial (BP), do que a busca completa com ordenação preliminar $\left(\mathrm{BC}_{-} 1\right)$ e, principalmente do que a busca com a regra de Armijo (AR), ou seja, o tempo computacional foi favorável ao método dual simplex com busca linear por partes utilizando o segundo procedimento de ordenação. Fica evidente a superioridade de se introduzir algum tipo de busca, pois o método dual simplex usual (SB) foi extremamente inferior em relação ao outros.

\subsubsection{Problemas Esparsos}

Nesta seção apresentamos os resultados obtidos para uma classe esparsa de problemas, para os quais a matriz de restrição é da forma escada. (Veja Figura 7.1). 
Nas Tabelas 7.13 e 7.15 estão os resultados obtidos para o método dual simplex com busca completa com ordenação preliminar (BC_1) e para o método dual simplex usual (SB) c nas Tabclas 7.16 e 7.18 estão os resultados para $\mathrm{BC}_{-} 1$ e para a regra de Armijo (AR), com a matriz de restrições tendo 4 blocos c 20 blocos, respectivamente. O símbolo \% indica a porcentagem do número de elementos diferentes de zero na matriz de restrições definida pelos blocos e, ce indica o número de colunas em comum para cada 2 blocos consecutivos, aproximadamente $20 \%$.

Tabela 7.13 - Busca Completa_ $1 \times$ Sem Busca - Problemas Esparsos 4 Blocos

\begin{tabular}{|c|c|r|r|r|r|r|r|}
\hline & & & \multicolumn{2}{c|}{ iteraçóes } & \multicolumn{2}{c|}{ tempo } \\
\hline exemplar & $c c$ & $\%$ & $m^{\prime} \times n$ & $B C-1$ & \multicolumn{1}{c|}{$S B$} & $B C-1$ & \multicolumn{1}{c|}{$S B$} \\
\hline 1 & 5 & 28 & $\mathbf{1 0 0 \times 1 0 1}$ & 128.9 & 374.2 & 0.06 & 0.10 \\
\hline 2 & 5 & 28 & $\mathbf{2 0 0 \times 1 0 1}$ & 209.5 & 615.1 & 0.11 & 0.26 \\
\hline 3 & 5 & 28 & $\mathbf{3 0 0 \times 1 0 1}$ & 259.6 & 793.1 & 0.13 & 0.43 \\
\hline 4 & 11 & 29 & $\mathbf{2 0 0 \times 2 0 3}$ & 343.2 & 1295.0 & 0.60 & 2.41 \\
\hline 5 & 11 & 29 & $\mathbf{3 0 0 \times 2 0 3}$ & 428.4 & 1747.6 & 0.98 & 3.61 \\
\hline 6 & 23 & 29 & $\mathbf{2 0 \times 4 0 3}$ & 20.2 & 101.8 & 0.09 & 0.28 \\
\hline 7 & 11 & 29 & $\mathbf{1 0 0 \times 2 0 3}$ & 93.2 & 248.7 & 0.17 & 0.40 \\
\hline 8 & 23 & 29 & $\mathbf{1 0 0 \times 4 0 3}$ & 95.6 & 335.7 & 0.62 & 1.18 \\
\hline 9 & 23 & 29 & $\mathbf{2 0 0 \times 4 0 3}$ & 221.6 & 666.8 & 2.47 & 8.90 \\
\hline 10 & 11 & 29 & $\mathbf{4 0 0 \times 2 0 3}$ & 522.8 & 2180.2 & 1.77 & 5.70 \\
\hline 11 & 23 & 29 & $\mathbf{4 0 0 \times 4 0 3}$ & 908.3 & 4284.8 & 8.99 & 33.4 \\
\hline & & & Média & 293.7 & $\mathbf{1 1 4 9 . 3}$ & $\mathbf{1 . 4 5}$ & $\mathbf{5 . 1 5}$ \\
\hline
\end{tabular}

A Tabela 7.13 mostra que, na média, o método dual simplex usual realizou 3.91 mais iterações do que o método dual simplex com a busca unidimensional completa e gastando 3.55 mais tempo. Note que a maior diferença para este caso, é observada para o exemplar 6 , $\mathrm{cm}$ que o número de variáveis é muito maior do que o número de restrições. Ou seja, mesmo para esta classe de problemas esparsos a busca unidimensional, faz uma diferença muito grande, apresentando um desempenho ainda melhor do que em problemas densos (veja Tabela 7.6).

Observa-se com relação ao caso denso (veja Tabela 7.6), que para os exemplares 3,5 e 10 $\left(m^{\prime}>n\right)$ o número de iterações é maior para os problemas esparsos com 4 blocos. Isto talvez seja explicado por ocorrer várias restrições redundantes para o caso denso, o que não deve acontecer para a estrutura escada.

Note também nas Tabelas 7.6 e 7.12, como o número de restrições $(m-n)$ tem maior impacto no número de itcraçõcs. Veja, por exemplo, os exemplares 9 e 10 da Tabela $7.6(200 \times 400$ e $400 \times 200$, respectivamente). Entretanto, o tempo computacional é bem menor para o exemplar 10, pois as matrizes básicas são da ordem de $200 \times 200$ enquanto para o exemplar 9 as matrizes básicas 
são da ordem $400 \times 400$. Obviamente, estas matrizes $400 \times 400$ têm uma estrutura particular que poderia ser explorada.

Na Tabcla 7.14 estão os resultados para a busca completa (BC_1) e para a regra de $\Lambda$ rmijo (AR).

\begin{tabular}{|c|c|c|c|c|c|c|c|}
\hline \multirow[b]{2}{*}{ exemplar } & \multirow[b]{2}{*}{$c c$} & \multirow[b]{2}{*}{$\%$} & \multirow[b]{2}{*}{$m^{\circ} \times n$} & \multicolumn{2}{|c|}{ iterações } & \multicolumn{2}{|c|}{ Tempo } \\
\hline & & & & $B C_{-} 1$ & $\overline{A R}$ & $B C_{1} 1$ & $A R$ \\
\hline 1 & 5 & 28 & $100 \times 101$ & 128.9 & 173.7 & 0.06 & 0.06 \\
\hline 2 & 5 & 28 & $200 \times 101$ & 209.5 & 269.9 & 0.11 & 0.13 \\
\hline 3 & 5 & 28 & $300 \times 101$ & 259.6 & 360.7 & 0.13 & $0 . \overline{19}$ \\
\hline 4 & 11 & 29 & $200 \times 203$ & 343.2 & 504.2 & 0.60 & 0.92 \\
\hline 5 & 11 & 29 & $300 \times 203$ & 428.4 & 709.9 & 0.98 & 1.55 \\
\hline 6 & 23 & 29 & $20 \times 403$ & 20.2 & 27.4 & 0.09 & 0.10 \\
\hline 7 & 11 & 29 & $100 \times 203$ & 93.2 & 103.4 & 0.17 & 0.29 \\
\hline 8 & 23 & 29 & $100 \times 403$ & 95.6 & 117.8 & 0.62 & 0.69 \\
\hline 9 & 23 & 29 & $200 \times 403$ & 221.6 & 278.1 & 2.47 & 4.57 \\
\hline 10 & 11 & 29 & $400 \times 203$ & 522.8 & 910.3 & 1.77 & 2.69 \\
\hline 11 & 23 & 29 & $400 \times 403$ & 908.3 & 1639.5 & $8 . \overline{99}$ & 14.89 \\
\hline & & & Média & 293.7 & 463.1 & 1.45 & 2.31 \\
\hline
\end{tabular}

Observamos que a busca completa (BC_1) foi ainda muito superior à regra de Armijo, apesar da diferença ter diminuído $\mathrm{em}$ relação ao caso denso (veja Tabela 7.8). A busca fez $\mathrm{cm}$ média 35\% menos iterações do que a regra de Armijo.

A Tabela 7.15 apresenta os resultados para a matriz de restrições tendo agora 20 blocos.

Tabela 7.15 - Busca Completa $1 \times$ Sem Busca - Problemas Esparsos 20 Blocos

\begin{tabular}{|c|c|c|c|c|c|c|c|}
\hline & & & & \multicolumn{2}{|c|}{ iterações } & \multicolumn{2}{|c|}{ Tempo } \\
\hline exemplar & $c c$ & $\%$ & $m^{\prime} \times n$ & $B C_{1} 1$ & $S B$ & $B C_{1} 1$ & $S B$ \\
\hline 1 & 1 & 5 & $100 \times 101$ & 87.1 & 145.6 & 0.05 & 0.07 \\
\hline 2 & 1 & 5 & $200 \times 101$ & 217.7 & 550.7 & 0.18 & 0.29 \\
\hline 3 & 1 & 5 & $300 \times 101$ & 272.9 & 735.0 & 0.26 & 0.52 \\
\hline 4 & 2 & 5 & $200 \times 202$ & 207.1 & $430 . \overline{1}$ & 1.45 & 2.42 \\
\hline 5 & 2 & 5 & $300 \times 202$ & 452.5 & 1618.5 & 1.61 & 5.01 \\
\hline 6 & 5 & 6 & $20 \times 405$ & 3.5 & 8.2 & 0.01 & 0.03 \\
\hline 7 & 2 & 5 & $100 \times 202$ & 15.3 & 26.5 & 0.04 & 0.12 \\
\hline 8 & 5 & 6 & $100 \times 405$ & 14.2 & 32.0 & 0.17 & 0.41 \\
\hline$\overline{9}$ & 5 & 6 & $200 \times 405$ & 35.6 & 67.0 & 0.60 & 1.15 \\
\hline 10 & 2 & 5 & $400 \times 202$ & 584.8 & 2121.8 & 2.13 & 6.05 \\
\hline$\overline{11}$ & 5 & 5 & $400 \times 405$ & 582.3 & 1779.0 & 6.80 & 14.5 \\
\hline & & & Média & 224.8 & 683.1 & 1.20 & 2.77 \\
\hline
\end{tabular}

Neste caso, muito mais esparso do que o caso com 4 blocos (veja Tabela 7.13), o número de iterações caiu significantemente para os dois métodos, o que indica que para problemas muito esparsos e com a matriz de restrições tendo vários blocos pequenos, o númcro de iterações tende a 
não passar de 1.5 do número de restrições para o método dual simplex com a busca e 5.4 para o método sem a busca. De uma maneira geral, para este caso também, o método dual simplex usual foi muito inferior em relação ao método DSBU.

$\Lambda$ Tabela 7.16 apresenta agora os resultados para a regra de Armijo e para a busca completa $\left(\mathrm{BC}_{-} 1\right)$.

\begin{tabular}{|c|c|c|c|c|c|c|c|}
\hline \multirow[b]{2}{*}{ exemplar } & \multirow[b]{2}{*}{$c c$} & \multirow[b]{2}{*}{$\%$} & \multirow[b]{2}{*}{$m^{\prime} \times n$} & \multicolumn{2}{|c|}{ iteraçōes } & \multicolumn{2}{|c|}{ Tempo } \\
\hline & & & & $B C_{1} 1$ & $A R$ & $B C_{1} 1$ & $A R$ \\
\hline 1 & 1 & 5 & $100 \times 101$ & 87.1 & 90.5 & 0.05 & 0.06 \\
\hline 2 & 1 & 5 & $200 \times 101$ & 217.7 & 276.1 & 0.18 & 0.20 \\
\hline 3 & 1 & 5 & $300 \times 101$ & 272.9 & 356.7 & 0.26 & 0.39 \\
\hline 4 & 2 & 5 & $200 \times 202$ & 207.1 & 224.5 & 1.45 & 1.70 \\
\hline 5 & 2 & 5 & $300 \times 202$ & 452.5 & 701.2 & 1.61 & 1.98 \\
\hline 6 & 5 & 6 & $20 \times 405$ & 3.5 & 4.0 & 0.01 & 0.01 \\
\hline 7 & 2 & 5 & $100 \times 202$ & 15.3 & 15.3 & 0.04 & 0.05 \\
\hline 8 & 5 & 6 & $100 \times 405$ & 14.2 & 15.4 & 0.17 & 0.18 \\
\hline 9 & 5 & 6 & $200 \times 405$ & 35.6 & 34.5 & 0.60 & 0.57 \\
\hline 10 & 2 & 5 & $400 \times 202$ & 584.8 & 867.9 & 2.13 & 3.78 \\
\hline 11 & 5 & 5 & $400 \times 405$ & 582.3 & 722.3 & 6.80 & 12.67 \\
\hline & & & Média & 224.8 & 300.76 & 1.41 & 1.96 \\
\hline
\end{tabular}

Observamos que, para este caso muito mais esparso, a regra de Armijo diminuiu consideravelmente a desvantagem em relação a BC_1 (vcja Tabela 7.9), conseguindo em alguns casos descmpenho equivalente a busca completa, veja os exemplares 1,7.8 e 9. Contudo, a busca completa foi ainda supcrior, fazendo em média $25 \%$ menos itcraçōes.

$\Lambda$ seguir nas Tabelas 7.17 c 7.18 apresentamos os resultados para o tempo de resolução $\mathrm{c}$ ordenação (com busca preliminar- $\mathrm{BC}_{-} 1$ ) para a matriz com 4 e 20 blocos respectivamente. onde $T T$ indica o tempo de resolução e $T O$ o tempo de ordenação para todas as iterações.

Tabela $7.17-$ Tempo Total $\times$ Tempo de Ordenaçăo

\begin{tabular}{|c|c|c|c|c|c|}
\hline & & & & \multicolumn{2}{|c|}{ tempo } \\
\hline exemplar & $c c$ & $\%$ & $\boldsymbol{m}^{\prime} \times \boldsymbol{n}$ & $T T$ & TO \\
\hline 1 & 5 & 28 & $100 \times 101$ & 0.06 & 0.00 \\
\hline 2 & 5 & 28 & $200 \times 101$ & 0.11 & 0.01 \\
\hline 3 & 5 & 28 & $\mathbf{3 0 0 \times 1 0 1}$ & 0.13 & 0.02 \\
\hline 4 & 11 & 29 & $200 \times 203$ & 0.60 & 0.03 \\
\hline 5 & 11 & 29 & $300 \times 203$ & 0.98 & 0.04 \\
\hline 6 & 23 & 29 & $20 \times 403$ & 0.09 & 0.00 \\
\hline 7 & 11 & 29 & $100 \times 203$ & 0.17 & 0.00 \\
\hline 8 & 23 & 29 & $100 \times 403$ & 0.62 & 0.01 \\
\hline 9 & 23 & 29 & $200 \times 403$ & 2.47 & 0.02 \\
\hline 10 & 11 & 29 & $\mathbf{4 0 0 \times 2 0 3}$ & 1.77 & 0.01 \\
\hline 11 & 23 & 29 & $\mathbf{4 0 0 \times 4 0 3}$ & 8.99 & 0.10 \\
\hline & & & Média & 1.45 & 0.02 \\
\hline \hline
\end{tabular}


Para este caso em que a matriz de restrições tem 4 blocos, o tempo de ordenação foi, em média, inferior a $2 \%$ do tempo total e, portanto, para problemas esparsos o tempo de ordenação é também irrelevante para o desempenho do método dual simplex com busca unidimensional.

Na Tabela 7.18 são exibidos os resultados dos tempos de ordenação e resolução para o caso da matriz de restrições tendo agora 20 blocos.

Tabela 7.18 - Tempo Total $\times$ Tempo de Ordenação

\begin{tabular}{|c|c|c|c|c|c|}
\hline & & & \multicolumn{3}{|c|}{ tempo } \\
\hline exemplar & $c c$ & $\%$ & $m^{\circ} \times n$ & $T T$ & $T O$ \\
\hline 1 & 1 & 5 & $100 \times 101$ & 0.05 & 0.00 \\
\hline 2 & 1 & 5 & $200 \times 101$ & 0.18 & 0.01 \\
\hline 3 & 1 & 5 & $300 \times 101$ & 0.26 & 0.01 \\
\hline 4 & 2 & 5 & $200 \times 202$ & 1.66 & 0.00 \\
\hline 5 & 2 & 5 & $300 \times 202$ & 1.61 & 0.02 \\
\hline 6 & 5 & 6 & $20 \times 405$ & 0.01 & 0.00 \\
\hline 7 & 2 & 5 & $100 \times 202$ & 0.04 & 0.00 \\
\hline 8 & 5 & 6 & $100 \times 405$ & 0.17 & 0.00 \\
\hline 9 & 5 & 6 & $\mathbf{2 0 0 \times 4 0 5}$ & 0.60 & 0.00 \\
\hline 10 & 2 & 5 & $\mathbf{4 0 0 \times 2 0 2}$ & 2.13 & 0.01 \\
\hline 11 & 5 & 5 & $\mathbf{4 0 0 \times 4 0 5}$ & 8.80 & 0.02 \\
\hline & & & Média & 1.41 & 0.00 \\
\hline
\end{tabular}

O mesmo pode ser observado da Tabela 7.18 para o caso anterior (veja Tabela 7.17). Ou scja, para os dois casos esparsos (matriz tendo 4 e 20 blocos) o tempo de ordenação foi insignificante em relação ao tempo total.

Agora, nas Tabela 7.19 e 7.20 são apresentados os resultados da busca completa com ordenação preliminar (BC_1) para as razões: $r / n$ e $\ell / r$, para os casos de 4 e 20 blocos da matriz de restrições, respectivamente. 
Tabela 7.19 - Número de $\delta$ 's e Número de $\ell ' s-4$ blocos

\begin{tabular}{|c|c|c|c|c|c|}
\hline exemplar & $c c$ & $\%$ & $m^{\prime} \times n$ & $r / n$ & $\ell / r$ \\
\hline 1 & 5 & 28 & $100 \times 101$ & 0.51 & 0.22 \\
\hline 2 & 5 & 28 & $200 \times 101$ & 0.49 & 0.27 \\
\hline 3 & 5 & 28 & $300 \times 101$ & 0.54 & 0.22 \\
\hline 4 & 11 & 29 & $200 \times 203$ & 0.49 & 0.22 \\
\hline 5 & 11 & 29 & $300 \times 203$ & 0.50 & 0.24 \\
\hline 6 & 23 & 29 & $20 \times 403$ & 0.13 & 0.62 \\
\hline 7 & 11 & 29 & $100 \times 203$ & 0.17 & 0.65 \\
\hline 8 & 23 & 29 & $100 \times 403$ & 0.17 & 0.53 \\
\hline 9 & 23 & 29 & $200 \times 403$ & 0.17 & 0.53 \\
\hline 10 & 11 & 29 & $400 \times 203$ & 0.49 & 0.98 \\
\hline \multirow[t]{2}{*}{11} & 23 & 29 & $400 \times 403$ & 0.50 & 0.98 \\
\hline & & & Média & 0.37 & 0.49 \\
\hline
\end{tabular}

Da Tabela 7.19, o número de $\delta^{\prime}$ s $(r)$ diminuiu em relação ao caso denso (veja Tabela 7.10) e pelos nossos testes notamos que esta fração tende a diminuir gradativamente com a esparsidade. Para o caso $\mathrm{cm}$ que o número de variáveis é maior do que o número de restrições, notamos que o número de $\delta$ 's diminuiu significantemente. Observamos ainda que, para os exemplares maiores 10 e 11 a razão $\not r$ (observada somente para a primeira iteração) foi muito alta, ou seja, o número de buscas cresceu em relação ao caso denso.

Tabela 7.20 - Número de $\delta$ 's e Número de $\ell ' s-20$ blocos

\begin{tabular}{|c|c|c|c|c|c|}
\hline exemplar & $c c$ & $\%$ & $m^{\prime} \times n$ & $r / n$ & $\ell / r$ \\
\hline 1 & 1 & 5 & $100 \times 101$ & 0.06 & 0.33 \\
\hline 2 & 1 & 5 & $200 \times 101$ & 0.49 & 0.43 \\
\hline 3 & 1 & 5 & $300 \times 101$ & 0.50 & 0.46 \\
\hline 4 & 2 & 5 & $200 \times 202$ & 0.05 & 0.20 \\
\hline 5 & 2 & 5 & $300 \times 202$ & 0.46 & 0.43 \\
\hline 6 & 5 & 6 & $20 \times 405$ & 0.03 & 0.30 \\
\hline 7 & 2 & 5 & $100 \times 202$ & 0.03 & 0.33 \\
\hline 8 & 5 & 6 & $100 \times 405$ & 0.04 & 0.25 \\
\hline 9 & 5 & 6 & $200 \times 405$ & 0.04 & 0.25 \\
\hline 10 & 2 & 5 & $400 \times 202$ & 0.50 & 0.86 \\
\hline \multirow[t]{2}{*}{11} & 5 & 5 & $400 \times 405$ & 0.06 & 0.11 \\
\hline & & & Média & 0.20 & 0.35 \\
\hline
\end{tabular}

Da Tabela 7.20, notamos que o número de $\delta$ 's diminuiu em relação ao caso de 4 blocos (Tabela 7.19), o que era de se esperar, pois este caso é muito mais esparso. Obscrva-se ainda que o número de $\delta$ 's foi próximo de $50 \%$ do valor de $n$ somente para os casos em que o númcro de 
restrições é maior do que o número de variáveis. Em relação à fração $₫ r$, obscrvamos que foi pequena para os exemplares 6,8 e 9 , tendo em vista que o número de deltas foi muito pequeno. Em relação ao caso denso, temos que o número de $\delta$ 's (i.e., $r$ ) diminuiu assim como a fração que determina a quantidade de $\delta^{\prime \prime}$ s utilizados na busca (i.e., $\emptyset$.

Diante destes resultados (Tabelas 7.19 e 7.20), utilizamos apenas a segunda modificação (veja capítulo 3) na busca unidimensional para os casos de 4 e 20 blocos da matriz de restrições, já que o númcro de $\delta^{\prime \prime}$ s foi pequeno. Os resultados são apresentados nas Tabclas 7.21 e 7.22.

\begin{tabular}{|c|c|c|c|c|c|}
\hline \multirow{2}{*}{ exemplar } & \multirow{2}{*}{$c c$} & \multirow{2}{*}{$\%$} & \multirow{2}{*}{$m^{\prime} \times n$} & \multicolumn{2}{|c|}{ tempo } \\
\hline & & & & $B C_{-} 1$ & $B C \_2$ \\
\hline 1 & 5 & 28 & $100 \times 101$ & 0.06 & 0.08 \\
\hline 2 & 5 & 28 & $200 \times 101$ & 0.11 & 0.10 \\
\hline 3 & 5 & 28 & $300 \times 101$ & 013 & 0.13 \\
\hline 4 & 11 & 29 & $200 \times 203$ & 0.60 & 0.57 \\
\hline 5 & 11 & 29 & $300 \times 203$ & 0.98 & 0.92 \\
\hline 6 & 23 & 29 & $20 \times 403$ & 0.09 & 0.09 \\
\hline 7 & 11 & 29 & $100 \times 203$ & 0.17 & 0.18 \\
\hline 8 & 23 & 29 & $100 \times 403$ & 0.62 & 0.61 \\
\hline 9 & 23 & 29 & $200 \times 403$ & 2.47 & 2.48 \\
\hline 10 & 11 & 29 & $400 \times 203$ & 1.77 & 1.69 \\
\hline 11 & 23 & 29 & $400 \times 403$ & 8.99 & 8.20 \\
\hline & & & Média & 1.45 & 1.36 \\
\hline
\end{tabular}

Neste caso o método dual simplex (BC_2) foi ligeiramente melhor do que o método dual simplex (BC_1). Note que a maior diferença é observada para o exemplar 11 de maior dimensão. Logo é de se esperar que o procedimento (BC_2) tenha um desempenho mais promissor para problemas de grande porte. 


\begin{tabular}{|c|c|c|c|c|c|}
\hline \multirow[b]{2}{*}{ exemplar } & \multirow[b]{2}{*}{$c c$} & \multirow[b]{2}{*}{$\%$} & \multirow[b]{2}{*}{$m \times n$} & \multicolumn{2}{|c|}{ tempo } \\
\hline & & & & $B C_{-} 1$ & $B_{2} 2$ \\
\hline 1 & 1 & 5 & $100 \times 101$ & 0.05 & 0.05 \\
\hline 2 & 1 & 5 & $200 \times 101$ & 0.18 & 0.17 \\
\hline 3 & 1 & 5 & $300 \times 101$ & 0.26 & 0.23 \\
\hline 4 & 2 & 5 & $200 \times 202$ & 1.66 & 1.65 \\
\hline 5 & 2 & 5 & $300 \times 202$ & 1.61 & 1.52 \\
\hline 6 & 5 & 6 & $20 \times 405$ & 0.01 & 0.01 \\
\hline 7 & 2 & 5 & $100 \times 202$ & 0.04 & 0.04 \\
\hline 8 & 5 & 6 & $100 \times 405$ & 0.17 & 0.18 \\
\hline 9 & 5 & 6 & $200 \times 405$ & 0.60 & 0.61 \\
\hline 10 & 2 & 5 & $400 \times 202$ & 2.13 & 2.01 \\
\hline 11 & 5 & 5 & $400 \times 405$ & 8.80 & 8.92 \\
\hline & & & Média & 1.41 & 1.39 \\
\hline
\end{tabular}

Notamos da Tabela 7.22 que na média houve praticamente um empate para os dois procedimentos de busca exata. Com uma ligeira vantagem para BC_2 na maioria dos excmplares. mas nada significante.

Contudo, podemos observar que o procedimento BC_2 teve um bom desempenho para problemas densos e para problemas com a matriz A tendo 4 Blocos, no entanto esta vantagem foi menos acentuada para o caso mais esparso, ou seja, a matriz A com 20 blocos e com densidade em torno de $6 \%$, pois o número de $\delta^{\prime \prime}$ s é menor em relação ao número de variáveis

Com relação a outras técnicas de ordenação, como relatado anteriormente (caso denso), realizamos os testes com o método de ordenação heapsort, mas não obtivemos uma melhora significante. E nota se ainda que, o efeito da ordenação tem pouco impacto no tempo total.

\subsection{Problemas Degenerados}

Nesta seção apresentamos os resultados para o método dual simplex com busca unidimensional DSBL da seção anterior, utilizando a forma produto da inversa para resolver alguns problemas degenerados da Netlib e outros gerados aleatoriamente na forma canalizada (forma (3.1), veja capítulo 3). Os problemas da Netlib que trabalhamos são problemas pequenos mas são problemas dificeis de serem resolvidos, no sentido que são muitos sensíveis a qualquer perturbação. Para o gerador utilizado, $80 \%$ dos elementos do vetor custo foram iguais a zero, de tal forma que várias soluções básicas duais sejam degeneradas.

A Tabela 7.23 apresenta os resultados obtidos para os problemas da Netlib para o método DSBU usual (sem nenhum procedimento anti-degeneração) e com os aplicativos compulacionais 
CPLEX e XPRESS (veja apêndice $\Lambda$ ), utilizando-se a regra de Dantzig (ou regra usual). O símbolo $\%$ representa a porcentagem de elementos não-nulos (ou densidade como usado anteriormente) na matriz de restrições que definida por $m$ 'restrições e $n$ variáveis. Para o pacote CPLEX 7.5, o préprocessador (veja apêndice A) não foi utilizado, para que a comparação seja 'mais justa'. Vale observar que o pré-processamento ć uma ferramenta poderosa para eliminar redundâncias (por conseguinte, degeneração), fixxar variávcis, apertar limitantes de modo que o problema resultante é mais fácil de ser resolvido. Por não ter sido explorado nesta tese, 'desligamos' o pré-processamento dos pacotes.

Tabela 7.23 - Problemas da Netlib*

\begin{tabular}{|c|c|c|c|c|c|c|c|c|}
\hline \multicolumn{3}{|c|}{ problemas } & \multicolumn{2}{c|}{ DSBU (usual) } & \multicolumn{2}{c|}{ XPRESS } & \multicolumn{2}{c|}{ CPLEX } \\
\hline nome & $\%$ & $\boldsymbol{m}^{\prime} \times \boldsymbol{n}$ & iteração & tempo & iteração & tempo & iteração & tempo \\
\hline afiro & 8.30 & $\mathbf{2 5 \times 3 9}$ & 18 & 0.00 & 15 & 0.01 & 11 & 0.00 \\
\hline adlitlle & 2.49 & $\mathbf{5 3 \times 1 9 6}$ & 109 & 0.10 & 32 & 0.02 & 125 & 0.00 \\
\hline $\boldsymbol{k b 2}$ & 12.79 & $\mathbf{4 3 \times 5 2}$ & 227 & 0.01 & 20 & 0.01 & 28 & 0.00 \\
\hline sc50a & 5.52 & $\mathbf{4 9 \times 4 8}$ & 52 & 0.00 & 59 & 0.01 & 45 & 0.00 \\
\hline sc50b & 5.12 & $\mathbf{4 8 \times 4 8}$ & 50 & 0.00 & 56 & 0.02 & 53 & 0.00 \\
\hline
\end{tabular}

*A biblioteca Netlib possui diversos problemas, porém estes foram os únicos que conseguimos 'limpar' ruídos (apêndice $A$ )

Podemos observar que o método DSBU (usual) teve o pior desempenho, sendo que o pacote CPLEX foi na média ligeiramente o melhor, no entanto o aplicativo XPRESS realizou apenas 32 iterações para o problema adlittle, um ganho muito significante.

$\mathrm{Na}$ Tabela 7.24 reportamos o número de iterações para o método DSBU usual, DSBU com o método expansão para tratar degeneração (veja capítulo 5) e para o CPLEX, sendo que o número de iterações é relatado também para a regra Dantzig Normalizada (DN), descrita no capítulo 5 ..

Tabela 724 - Problemas da Netlib

\begin{tabular}{|c|c|c|c|c|c|c|}
\hline & \multicolumn{2}{|c|}{ DSBU usual } & \multicolumn{2}{c|}{ DSBU expansão } & \multicolumn{2}{c|}{ CPLEX } \\
\hline nome & $D$ & $D N$ & $D$ & $D N$ & $D$ & $D N$ \\
\hline afiro & 18 & 20 & 15 & 16 & 11 & 11 \\
\hline adlitlle & 109 & 77 & 108 & $138(94)^{*}$ & 125 & 86 \\
\hline kb2 & 227 & 126 & 31 & 144 & 28 & 29 \\
\hline sc50a & 52 & 48 & 51 & 48 & 45 & 45 \\
\hline sc50b & 50 & 50 & 50 & 50 & 53 & 55 \\
\hline
\end{tabular}


*O valor entre parênteses é o número de iterações que foi obtido com uma pequena mudança $\mathrm{cm}$ um parâmetro do método de expansão, mas cssa mudança foi testada para os outros problemas, sem aprescntar melhora.

Podemos observar da Tabela 7.24, que a regra de Dantzig Normalizada foi muito superior à regra usual somente para os problemas adlittle e $k b 2$, com o método DSBU usual, ocorrendo o oposto para DSBU-expansâo. Observamos tambćm que as regras D (regra usual) e DN se mostraram praticamente equivalentes no CPLEX, com exceção do problema adlittle, realizando $70 \%$ das iterações da regra de Dantzig.

Contudo, o método de expansão fez uma pequena redução no número de iterações $\mathrm{cm}$ relação ao método DSBU usual, embora tenha piorado o desempenho do método DSBU com a regra DN para o problema adlittle. O tempo para DSBU com o método de expansão, foi praticamente o mesmo que DSBU usual.

$\mathrm{Na}$ próxima seção, apresentamos os resultados com a regra $\mathrm{DN} \mathrm{cm}$ problemas gerados aleatoriamente, para verificar seu desempenho.

As Tabelas 7.25 e 7.26 apresentam os resultados com DSBU usual e DSBU expansão, respectivamente, para os problemas degenerados, que foram gerados aleatoriamente, para que possamos comparar melhor se o método de expansão diminui a estagnação no número de iteraçñes.

Tabela 7.25 - Problemas degenerados resolvidos pelo DSBU usual

\begin{tabular}{|c|c|c|c|c|c|c|c|c|c|}
\hline \multicolumn{6}{|c|}{ problemas } & \multicolumn{2}{|c|}{ iterações } & \multicolumn{2}{|c|}{ tempo } \\
\hline$n b$ & $\overline{m 1}$ & $n 1$ & $\overline{c c}$ & $\%$ & $m^{\prime} \times n$ & $D$ & $D N$ & $D$ & $D N$ \\
\hline 20 & 1 & 20 & $\overline{0}$ & 5.00 & $20 \times 400$ & 19.8 & 20.3 & 0.11 & 0.16 \\
\hline 100 & 2 & 5 & 3 & 2.46 & $200 \times 203$ & 199.6 & 182.3 & 0.78 & 1.92 \\
\hline 25 & 8 & 16 & 4 & 5.26 & $200 \times 304$ & 36.3 & 34.4 & 0.40 & 0.56 \\
\hline 50 & 4 & 12 & 3 & 2.64 & $200 \times 453$ & 12.8 & 14.1 & 0.33 & 0.46 \\
\hline 200 & 2 & 6 & 4 & 1.48 & $400 \times 404$ & 532.2 & 478.2 & 24.6 & 39.6 \\
\hline
\end{tabular}

Da Tabela 7.25 , notamos que a regra DN teve no geral uma pequena vantagem em relação a regra usual, principalmente nos problemas de dimensão $400 \times 404$, em que cla realizou $10 \%$ menos iterações do que a regra D, mas por outro lado o tempo foi muito alto, devido aos cálculos extras (veja capítulo 5) que foram realizados em cada iteração. No entanto, é possível que uma implementação refinada da regra de Dantzig normalizada possa reduzir o tempo por iteração. 
Tabela 7.26 - Problemas degenerados resolvidos pelo DSBU expansão

\begin{tabular}{|c|c|c|c|c|c|c|c|c|c|}
\hline \multicolumn{6}{|c|}{ problemas } & \multicolumn{2}{|c|}{ iteraçōes } & \multicolumn{2}{|c|}{ tempo } \\
\hline$n b$ & $m 1$ & $n 1$ & $C C$ & $\%$ & $m^{\prime} \times n$ & $\bar{D}$ & $D N$ & $\bar{D}$ & $D N$ \\
\hline 20 & 1 & 20 & 0 & 5.0 & $20 \times 400$ & 19.8 & 20.3 & 0.11 & 0.17 \\
\hline 100 & 2 & 5 & 3 & 2.46 & $200 \times 203$ & 189.8 & 161.2 & 0.71 & 1.65 \\
\hline 25 & 8 & 16 & 4 & 5.26 & $200 \times 304$ & 31.8 & 30.3 & 0.36 & 0.51 \\
\hline 50 & 4 & 12 & 3 & 2.64 & $200 \times 453$ & 12.4 & 12.5 & 0.32 & 0.42 \\
\hline 200 & 2 & 6 & 4 & 1.48 & $400 \times 404$ & 486.0 & 405.2 & 18.63 & 23.18 \\
\hline
\end{tabular}

A Tabela 7.26 mostra que o método de expansão faz reduzir significantemente o número de iterações para estes problemas degenerados (veja a Tabela 7.25 para comparação) tanto para a regra usual quanto para a regra DN, como conseqüência, obviamente o tempo de resolução foi menor.

Esta redução fica mais evidente nos problemas $(400 \times 404)$, em que o número de iterações foi $10 \%$ e $15 \%$ menor (em relação DSBU usual) para as regras D e DN, respectivamente.

Diante destes resultados, utilizamos o pacote CPLEX para resolver esta mesma classe de problemas, cujos resultados estão na Tabela 7.27 .

\begin{tabular}{|c|c|c|c|c|c|c|c|c|c|}
\hline \multicolumn{6}{|c|}{ problemas } & \multicolumn{2}{|c|}{ iteração } & \multicolumn{2}{|c|}{ tempo } \\
\hline$n b$ & $m 1$ & $n 1$ & $c c$ & $\%$ & $m^{\prime} \times n$ & $\bar{D}$ & $D N$ & $D$ & $D N$ \\
\hline 20 & 1 & 20 & 0 & 5.00 & $20 \times 400$ & 73.5 & 73.2 & 0.00 & 0.00 \\
\hline 100 & 2 & 5 & 3 & 2.46 & $200 \times 203$ & 257.2 & 246.2 & 0.00 & 0.00 \\
\hline 25 & 8 & 16 & 4 & 5.26 & $200 \times 304$ & 50.1 & 49.5 & 0.00 & 0.00 \\
\hline 50 & 4 & 12 & 3 & 2.64 & $200 \times 453$ & 17.8 & 19.5 & 0.00 & 0.00 \\
\hline 200 & 2 & 6 & 4 & 1.48 & $400 \times 404$ & 603.4 & 512.9 & 0.04 & 0.03 \\
\hline
\end{tabular}

Notamos da Tabela 7.27 que, a regra DN para o CPLEX teve uma melhora significante somente para os problemas de dimensão $400 \times 404$, tendo seu desempenho equivalente a regra usual nos demais problemas.

Comparando os resultados das Tabelas 7.25, 7.26 e 7.27, lica clara a superioridade do método DSBU frente ao CPLEX, principalmente quando se faz o uso do método de expansão, embora o tempo não seja favorável. Por exemplo, para os problemas $20 \times 400$, o pacote CPLEX realizou aproximadamente 3 vezes mais iterações do que o método DSBU, logo é importante observar que nestes problemas tendo apenas 20 restrições, $80 \%$ dos custos são iguais a zero, caracterizando-se, assim, problemas muitos degenerados como os problemas $400 \times 404$, mas que para estes problemas não há uma diferença (de 3 vezes mais iterações) por se tratar de exemplares 
bem mais restrito, ou seja, a degeneração é um problema que deve ser tratado com métodos eficientes.

Segundo informações do manual do CPLEX 7.5 (veja apêndice A), problemas degencrados são tratados com perturbação, mas notamos que o método de expansão desenvolvido para o DSBI apresentou resultados melhores para os problemas degenerados gcrados.

Embora descrevemos o procedimento de perturbação (capítulo 5). o número de iterações foi ligeiramente menor do que o DSBU usual e, em muitos casos, as soluções para estes problemas foram infactíveis, logo não apresentamos tais resultados.

Portanto, fica evidente a necessidade de se utilizar algum procedimento anti-degeneração no método simplex para resolver problemas degenerados.

\subsection{O Método DSBU com Regra de Dantzig Normalizada}

Nesta seção, apresentamos os experimentos computacionais para a regra de Dantzig Normalizada para problemas densos e esparsos com o método DSBU.

A Tabela 7.28 apresenta os resultados obtidos para o método DSBU com as regras de Danzig e Dantzig normalizada, com quase $100 \%$ dos elementos na matriz de restrições diferentes de zero.

\begin{tabular}{|c|c|c|c|c|c|}
\hline & \multicolumn{2}{|c|}{ TSBU } & \multicolumn{2}{c|}{ DSBU } \\
\hline Exemplar & $\boldsymbol{m}^{\mathbf{2} \times \boldsymbol{n}}$ & $\boldsymbol{D N}$ & $\mathbf{t e m p o}$ & $\boldsymbol{D}$ & Tempo \\
\hline 1 & $\mathbf{1 0 0 \times \mathbf { 1 0 0 }}$ & 140.3 & 0.05 & 128.2 & 0.06 \\
\hline 2 & $\mathbf{2 0 0 \times \mathbf { 1 0 0 }}$ & 220.7 & 0.19 & 206.2 & 0.17 \\
\hline 3 & $\mathbf{3 0 0 \times \mathbf { 1 0 0 }}$ & 235.6 & 0.25 & 235.3 & 0.11 \\
\hline 4 & $\mathbf{2 0 0 \times \mathbf { 2 0 0 }}$ & 302.5 & 0.84 & 294.4 & 0.38 \\
\hline 5 & $\mathbf{3 0 0 \times \mathbf { 2 0 0 }}$ & 395.5 & 1.00 & 382.3 & 0.60 \\
\hline 6 & $\mathbf{2 0 \times \mathbf { 4 0 0 }}$ & 58.2 & 0.31 & 57.5 & 0.23 \\
\hline 7 & $\mathbf{1 0 0 \times \mathbf { 2 0 0 }}$ & 191.3 & 0.23 & 174.5 & 0.18 \\
\hline 8 & $\mathbf{1 0 0 \times 4 0 0}$ & 270.8 & 2.36 & 236.9 & 1.04 \\
\hline 9 & $\mathbf{2 0 0 \times 4 0 0}$ & 435.9 & 3.17 & 395.5 & 1.03 \\
\hline 10 & $\mathbf{4 0 0 \times \mathbf { 2 0 0 }}$ & 492.3 & 2.20 & 475.7 & 0.94 \\
\hline 11 & $\mathbf{4 0 0 \times 4 0 0}$ & 620.5 & 8.33 & 632.9 & 3.91 \\
\hline
\end{tabular}

Na Tabela 7.28, apenas no exemplar $11(400 \times 400)$ a regra de Dantzig Normalizada reduziu o número de iterações, enquanto o tempo computacional foi sensivelmente favorável à regra de Dantzig. 
Nas Tabelas 7.29 e 7.30, comparamos para estes problemas densos, o desempenho do método DSBU com o pacote CPLEX, utilizando-se as regras D e DN, respectivamente.

\begin{tabular}{|c|c|c|c|c|c|}
\hline \multirow[b]{2}{*}{ exemplar } & \multirow[b]{2}{*}{$m^{\prime} \times n$} & \multicolumn{2}{|c|}{$D S B U$} & \multicolumn{2}{|c|}{ CPLEX } \\
\hline & & $D N$ & tempo & $D N$ & tempo \\
\hline 1 & $100 \times 100$ & 140.3 & 0.05 & 213.7 & 0.09 \\
\hline 2 & $200 \times 100$ & 220.7 & 0.19 & 283.5 & 0.28 \\
\hline 3 & $300 \times 100$ & 235.6 & 0.25 & 333.4 & 0.48 \\
\hline 4 & $200 \times 200$ & 302.5 & 0.84 & 455.2 & 1.19 \\
\hline 5 & $300 \times 200$ & 395.5 & 1.00 & 690.1 & 2.20 \\
\hline 6 & $20 \times 400$ & 58.2 & 0.31 & 48.7 & 0.02 \\
\hline 7 & $100 \times 200$ & 191.3 & 0.23 & 256.4 & 0.19 \\
\hline 8 & $100 \times 400$ & 270.8 & 2.36 & 338.6 & 0.42 \\
\hline 9 & $200 \times 400$ & 435.9 & 3.17 & 663.4 & 2.20 \\
\hline 10 & $400 \times 200$ & 492.3 & 2.20 & 801.1 & 3.19 \\
\hline 11 & $400 \times 400$ & 620.5 & 8.33 & 1578.6 & 13.52 \\
\hline
\end{tabular}

A Tabela 7.29 permite algumas observações interessantes. Por se tratarem de excmplares densos, as estruturas de dados do DSBU e CPLEX tendem a ser equivalentes (i.e. a exploração da esparsidade fica sem efeito), de modo que a comparação dos desempenhos dos algoritmos são 'mais justas' (já que o efeito da estrutura de dados é insignificante).

Como já observado na seção 7.2, os resultados de Silva (2002) mostram o impacto sobre o desempenho em termos de tempo, quando a esparsidade é explorada. Assim, sem a influência da estrutura de dados, a Tabela 7.7 mostra que o algoritmo DSBU é superior ao algoritmo implementado no pacote CPLEX.

Os exemplares de dimensão $400 \times 400$ mostram que o número de iterações pode ser reduzido em quase 1/3. O tempo computacional não acompanha esta razão, pois há uma 'parte'esparsa que vale a pena ser ainda pesquisada. Por exemplo, os exemplares $20 \times 400$ (foi a única classe em que 0 DSBU fe $\% 25 \%$ de iterações a mais que o CPLEX; algo curioso, que também merece investigação), têm as bases duais $400 \times 400$, sendo que poucas colunas (no máximo 20) são densas, as demais são colunas da matriz identidade. Esta 'esparsidade interna' torna o CPLEX muito mais cficiente, como mostra a Tabela 7.29 .

$A$ classe de exemplos $100 \times 400$, isto é ainda mais realçado, pois DSBU fez aproximadamente $80 \%$ do número de itcrações do CPLEX, entretanto o tempo é mais do que 3 vezes o tempo do CPLEX.

Investigamos também se este mesmo comportamento pode ser observado para regra usual. A Tabela 7.30 apresenta os resultados obtidos. 
Tabela 7.30 - Problemas densos

\begin{tabular}{|c|c|c|c|c|c|}
\hline & & \multicolumn{2}{|c|}{$D S B U$} & \multicolumn{2}{|c|}{ CPLEX } \\
\hline exemplar & $m^{\prime} \times n$ & $D$ & tempo & $D$ & tempo \\
\hline 1 & $100 \times 100$ & 128.2 & 0.06 & 210.6 & 0.09 \\
\hline 2 & $200 \times 100$ & 206.2 & 0.17 & 350.2 & 0.30 \\
\hline 3 & $300 \times 100$ & 235.3 & 0.11 & 428.1 & 0.55 \\
\hline 4 & $200 \times 200$ & 294.4 & 0.38 & 556.2 & 1.01 \\
\hline 5 & $300 \times 200$ & 382.3 & 0.60 & 796.5 & 2.12 \\
\hline 6 & $20 \times 400$ & 57.5 & 0.23 & 64.3 & 0.02 \\
\hline 7 & $100 \times 200$ & 174.5 & 0.18 & 354.6 & 0.21 \\
\hline 8 & $100 \times 400$ & 236.9 & 1.04 & 407.1 & 0.48 \\
\hline 9 & $200 \times 400$ & 395.5 & 1.93 & 901.3 & 2.44 \\
\hline 10 & $400 \times 200$ & 475.7 & 0.94 & 990.6 & 3.01 \\
\hline 11 & $400 \times 400$ & 632.9 & 3.91 & 1549.8 & 10.01 \\
\hline
\end{tabular}

Da Tabela 7.30. notamos praticamente o mesmo 'cenário' mostrado pela Tabela 7.29, sendo que, para os exemplares $20 \times 400$, o númcro de iterações foi quase o mesmo para DSBU e para o CPLEX e, para os exemplares $100 \times 400$, o método DSBU realizou 58\% das iterações do CPLEX, mas com o dobro do tempo, já que a maioria das colunas básicas são colunas da matriz identidade. Esta 'csparsidade interna' deve ser alvo de pesquisa futura.

Diante dos resultados obtidos com a regra de Dantzig Normalizada, investigamos uma classe esparsa de problemas (forma escada com 4 blocos e 20 blocos para cada problema), para podemos observar mais uma vez o comportamento da regra DN frente a regra usual

Nas Tabelas 7.31 e 7.32 estão os resultados obtidos para as regras de Dantzig e Dantzig Normalizada, com a matri\% de restrições tendo 4 blocos e 20 blocos respectivamente, em que ce indica o número de colunas em comum para cada 2 blocos, aproximadamente $20 \%$.

Tabela 7.31-Dantzig $\times$ Dantzig Normalizada - Problemas Esparsos 4 Blocos

\begin{tabular}{|c|c|c|c|c|c|c|c|}
\hline \multirow[b]{2}{*}{ exemplar } & \multirow[b]{2}{*}{ CC } & \multirow[b]{2}{*}{$\%$} & \multirow[b]{2}{*}{$m^{\prime} \times n$} & \multicolumn{2}{|c|}{$D S B U$} & \multicolumn{2}{|c|}{$D S B U$} \\
\hline & & & & $D N$ & tempo & $D$ & tempo \\
\hline 1 & 5 & 28 & $100 \times 101$ & 136.6 & 0.10 & 144.30 & 0.06 \\
\hline 2 & 5 & 28 & $200 \times 101$ & 240.5 & 0.29 & 205.40 & 0.13 \\
\hline 3 & 5 & 28 & $300 \times 101$ & 253.4 & 0.48 & 242.70 & 0.22 \\
\hline 4 & 11 & 29 & $200 \times 203$ & 361.1 & 1.50 & 331.40 & 0.69 \\
\hline 5 & 11 & 29 & $300 \times 203$ & 480.8 & 2.60 & 427.50 & 1.20 \\
\hline 6 & 23 & 29 & $20 \times 403$ & 22.1 & 0.02 & 21.6 & 0.01 \\
\hline 7 & 11 & 29 & $100 \times 203$ & 94.1 & 0.55 & 94.9 & 0.32 \\
\hline 8 & 23 & 29 & $100 \times 403$ & 98.6 & 1.92 & 95.9 & 0.97 \\
\hline 9 & 23 & 29 & $200 \times 403$ & 231.5 & 10.4 & 223.2 & 5.66 \\
\hline 10 & 11 & 29 & $400 \times 203$ & 534.7 & 3.81 & 519.8 & 1.77 \\
\hline 11 & 23 & 29 & $400 \times 403$ & 740.1 & 20.2 & 877.4 & 11.15 \\
\hline
\end{tabular}


Podemos observar da Tabela 7.31 com relação ao caso denso (Tabela 7.28), que para as duas regras, o número de itcrações cresceu quando o número de restrições é maior que o número de variáveis e diminuiu quando se têm a sua situação oposta. Isto ocorre, pois devem existir muitas restrições redundantes para o caso denso, o que não deve acontecer para a estrutura escada.

Nota-se ainda, um menor número de iterações para regra de Dantzig, porém a diferença diminuiu com relação aos resultados da Tabela 7.28, com exceção do exemplar 11, em que a regra de Dantzig Normalizada realizou aproximadamente $15 \%$ menos iterações com relação a regra usual.

A Tabela 7.32 apresenta os resultados para a matriz de restrições tendo agora 20 blocos.

\begin{tabular}{|c|c|c|c|c|c|c|c|}
\hline \multirow[b]{2}{*}{ Exemplar } & \multirow[b]{2}{*}{$\mathrm{Cc}$} & \multirow[b]{2}{*}{$\%$} & \multirow[b]{2}{*}{$m^{\prime} \times n$} & \multicolumn{2}{|c|}{$D S B U$} & \multicolumn{2}{|c|}{$D S B U$} \\
\hline & & & & $D N$ & tempo & $D$ & tempo \\
\hline 1 & 1 & 5 & $100 \times 101$ & 86.5 & 0.17 & 87.9 & 0.05 \\
\hline 2 & 1 & 5 & $200 \times 101$ & 230.6 & 0.33 & 204.3 & 0.17 \\
\hline 3 & 1 & 5 & $300 \times 101$ & 270.3 & 0.56 & 257.8 & 0.27 \\
\hline 4 & 2 & 5 & $200 \times 202$ & 199.7 & 2.01 & 211.5 & 1.41 \\
\hline 5 & 2 & 5 & $300 \times 202$ & 400.9 & 2.78 & 472.8 & 1.65 \\
\hline 6 & 5 & 6 & $20 \times 405$ & 3.4 & 0.02 & 3.3 & 0.03 \\
\hline 7 & 2 & 5 & $100 \times 202$ & 13.6 & 0.06 & 14.3 & 0.01 \\
\hline 8 & 5 & 6 & $100 \times 405$ & 15.1 & 0.25 & 14.8 & 0.17 \\
\hline 9 & 5 & 6 & $200 \times 405$ & 34.6 & 0.85 & 33.6 & 0.60 \\
\hline 10 & 2 & 5 & $400 \times 202$ & 560.5 & 5.61 & 550.2 & 2.40 \\
\hline 11 & 5 & 5 & $400 \times 405$ & 554.2 & 31.4 & 582.9 & 13.35 \\
\hline
\end{tabular}

Neste caso, muito mais esparso do que o caso com 4 blocos, o número de iterações caiu significantemente para as duas regras, o que indica que para problemas muito esparsos e com a matriz de restrições tendo vários blocos pequenos, o número de iterações tende a não passar de duas vezes do número de restrições. De uma maneira geral, para este caso também, a regra de Dantzig Normalizada foi inferior no número de iterações e no tempo computacional. A Figura 7.3 fornece o gráfico para o número de iterações. 


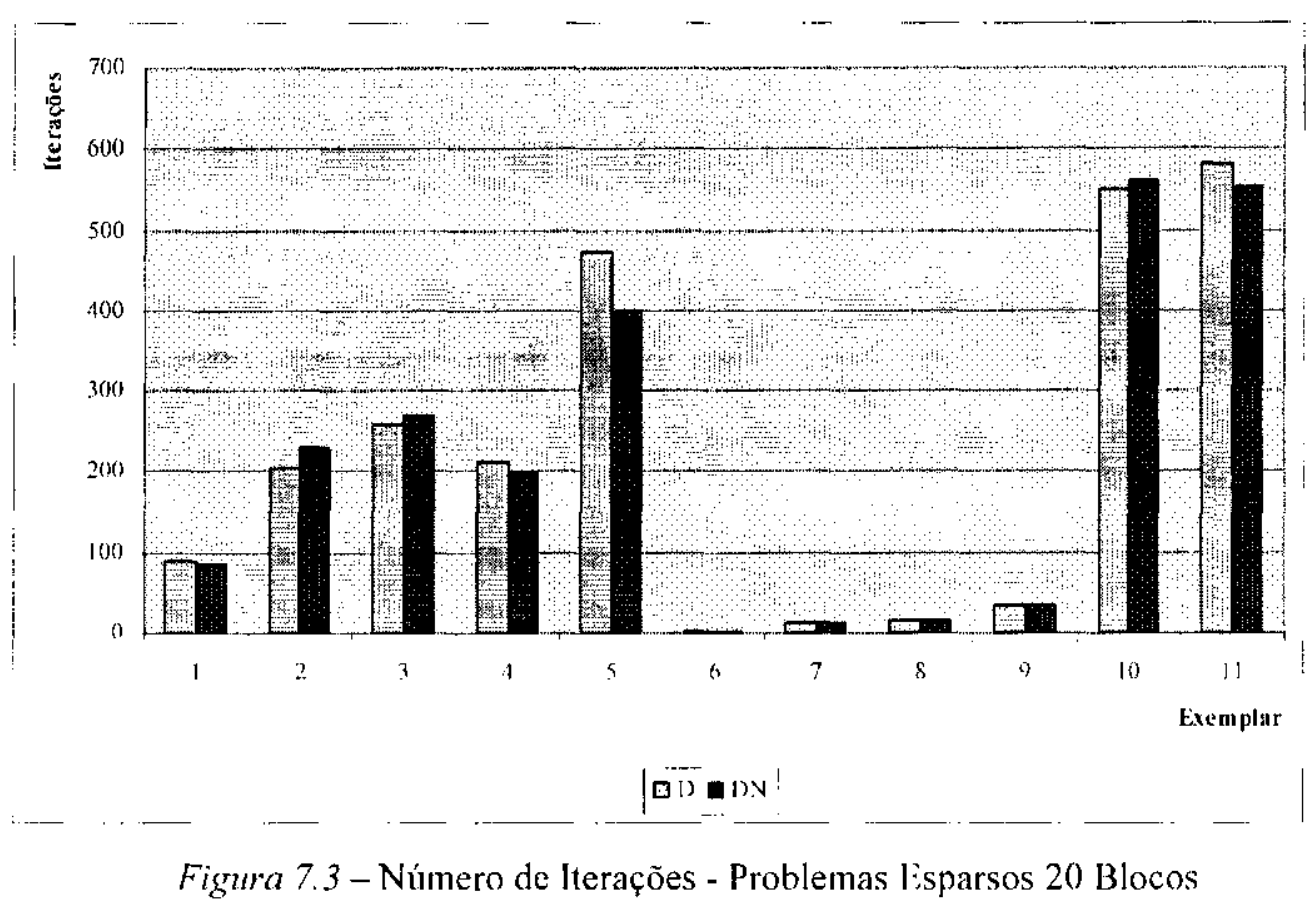

Da Figura 7.3, note que para os cxemplares $6,7,8$ e 9 em que o número de variáveis é pelo menos 2 vezes o número de restrições, o número de iteraçôes foi muito baixo c quando se tem a situação oposta, as iterações aumentam. mas não ultrapassando 1,5 do número de restrições.

Realizamos testes com os mesmos problemas com estrutura escada com 4 e 20 blocos para o pacote CPI.EX, mas iremos apresentar somente os resultados obtidos com os problemas tendo 20 blocos na Tabela 7.33 , pois os resultados foram similares.

Tabela 7.33 - Problemas esparsos para caso de 20 Blocos

\begin{tabular}{|c|c|c|c|c|c|c|c|c|}
\hline \multirow{2}{*}{$\frac{\text { exemp/ar }}{m^{\circ} \times n}$} & \multicolumn{2}{|c|}{ DSBU-iteração } & \multicolumn{2}{|c|}{$D S B U$-tempo } & \multicolumn{2}{|c|}{ CPLEX-iteração } & \multicolumn{2}{|c|}{ CPLEX-tempo } \\
\hline & $D$ & $D N$ & $\bar{D}$ & $D N$ & $D$ & $D N$ & $D$ & $D N$ \\
\hline $100 \times 101$ & 87.9 & 86.5 & 0.05 & 0.17 & 99.5 & 96.3 & 0.01 & 0.00 \\
\hline $200 \times 101$ & 204.3 & 230.6 & 0.17 & 0.33 & 368.5 & 256.3 & 0.07 & 0.07 \\
\hline $300 \times 101$ & 257.8 & 270.3 & 0.27 & 0.56 & 483.6 & 304.6 & 0.20 & 0.18 \\
\hline $200 \times 202$ & 211.5 & 199.7 & 1.41 & 2.01 & 268.5 & 231.8 & 0.02 & 0.02 \\
\hline $300 \times 202$ & 472.8 & 400.9 & 1.65 & 2.78 & 758.0 & 567.5 & 0.40 & 0.39 \\
\hline $20 \times 405$ & 3.3 & 3.4 & 0.03 & 0.02 & 2.0 & 2.0 & 0.00 & 0.00 \\
\hline $100 \times 202$ & 14.3 & 13.6 & 0.01 & 0.06 & 15.1 & 13.8 & 0.00 & 0.00 \\
\hline $100 \times 405$ & $14 . \overline{8}$ & 15.1 & 0.17 & 0.25 & 14.1 & 12.1 & 0.00 & 0.00 \\
\hline $200 \times 405$ & 33.6 & 34.6 & 0.60 & 0.85 & 34.1 & 31.3 & 0.00 & 0.00 \\
\hline $400 \times 202$ & 550.2 & 560.5 & 2.40 & 5.61 & 1055.2 & 661.5 & 1.13 & 0.90 \\
\hline $400 \times 405$ & 582 & 554.2 & 13.35 & 31.4 & 803.1 & 649.1 & 0.20 & 0.21 \\
\hline
\end{tabular}

De novo pode ser observado que a estrutura de dados faz diferença no tempo (como relatado para as Tabelas 7.29 e 7.30), pois para o método DSBU com a regra usual ou de Dantzig 
normalizada o tempo médio aumenta (proporcional ao número de iterações) quando os problemas passam da classe de 4 blocos para 20 blocos, ao contrário do que ocorre para o CPLEX

Realizamos também, testes com outros exemplares para cada tamanho do problema, para o método DSBU com a regra usual (D) e para o CPLEX, sendo que o número de blocos, linhas e colunas (de cada bloco) tivessem uma variação. $\Lambda$ Tabela 7.34 exibe os resultados.

Tabela 7.34 - Problemas esparsos com vários tamanhos de blocos

\begin{tabular}{|c|c|c|c|c|c|c|c|c|c|}
\hline \multicolumn{6}{|c|}{ Problema } & \multicolumn{2}{|c|}{$D S B U=D$} & \multicolumn{2}{|c|}{$\overline{C P L E X}-D$} \\
\hline$n b$ & $m 1$ & $n 1$ & $c c$ & $\%$ & $m^{\prime} \times n$ & iteração & tempo & iteração & tempo \\
\hline 6 & 2 & 2 & 0 & 16.66 & $12 \times 12$ & 9.1 & 0.00 & 8.3 & 0.00 \\
\hline 10 & 2 & 2 & 0 & 10.66 & $20 \times 20$ & 14.8 & 0.00 & 12.3 & 0.00 \\
\hline 12 & 4 & 4 & 0 & 8.33 & $48 \times 48$ & 38.7 & 0.01 & 38.0 & 0.01 \\
\hline 20 & 1 & 20 & 2 & 5.33 & $20 \times 362$ & 2.1 & 0.03 & 1.89 & 0.00 \\
\hline 100 & 2 & 3 & 1 & 1.49 & $200 \times 201$ & 149.1 & 1.61 & 156.8 & 0.02 \\
\hline 100 & 2 & 4 & 2 & 1.33 & $200 \times 202$ & 171.2 & 2.40 & 174.6 & 0.02 \\
\hline 200 & 2 & 2 & 0 & 0.73 & $400 \times 400$ & 277.3 & 9.01 & 275.6 & 0.02 \\
\hline
\end{tabular}

Da Tabela 7.34 notamos que o método dual simplex com busca unidimensional tem desempenho, em termos do número de iterações, equivalentes ao CPLEX.

Comparamos também o desempenho da regra de DN para esta mesma classe de problemas da Tabela 7.34, mas as conclusões foram semelhantes a este caso.

De uma maneira geral, a expectativa era do melhor desempenho para a Dantzig Normalizada com relação à regra de Dantzig. Mas, pelos resultados obtidos com os exemplares nas diferentes classes, isto ć um indicativo que para problemas na forma geral de pequeno porte a regra de Dantzig Normalizada é inferior à regra usual e em alguns casos equivalente, cmbora o trabalho de Forrest $\mathrm{e}$ Goldfarb (1992) aponta uma clara superioridade da regra de Dantzig Normaliza com relação à regra usual, em alguns problemas na Netlib. Porém, selecionamos alguns problemas da Netlib que não estão no trabalho de Forrest e Goldfarb (1992) e utilizamos o CPLEX para resolver esses problemas, cujos resultados estão na Tabela 7.35 . 
Tabela 7.35 - Problemas da Netlib resolvidos pelo CPLEX

\begin{tabular}{|c|c|c|c|c|c|}
\hline \multicolumn{2}{|c|}{ Problema } & \multicolumn{2}{|c|}{ iterações } & \multicolumn{2}{|c|}{ tempo } \\
\hline nome & $m^{\prime} \times n$ & $D N$ & $D$ & $D N$ & $D$ \\
\hline$a g g$ & $489 \times 163$ & 121 & 145 & 0.01 & 0.03 \\
\hline modszk1 & $688 \times 1620$ & 118 & 129 & 0.02 & $0 . \overline{02}$ \\
\hline maros & $847 \times 1443$ & 1457 & 1955 & 0.25 & 0.17 \\
\hline maros-r7 & $3137 \times 9408$ & 5468 & 6696 & 18.97 & 12.91 \\
\hline elamacro & $401 \times 688$ & 782 & 676 & 0.08 & 0.03 \\
\hline S ganges & $1310 \times 1681$ & 323 & 319 & 0.03 & 0.01 \\
\hline , osa 07 & $1118 \times 23949$ & 623 & 426 & 0.78 & 0.34 \\
\hline $0 s a-14$ & $237 \times 52460$ & 1926 & 989 & 6.42 & 2.25 \\
\hline $0 s a-30$ & $4350 \times 100024$ & 4801 & 1836 & 42.30 & 8.78 \\
\hline
\end{tabular}

Notamos Tabela 7.35 que, para os dois primeiros problemas o número de iterações para a regra DN teve uma pequena vantagem sobre a regra usual, mas a superioridade da regra de Dantzig normaliza, do ponto de vista do número de iterações, é observada somente neste caso nos problemas maros e maros-r7, embora o tempo seja $\mathrm{em}$ média $50 \%$ a mais, um fato curioso para tais problemas. Entretanto a regra $\mathrm{DN}_{2}$ teve um desempenho inferior à regra usual, principalmente para os dois últimos problemas, realizando mais do que o dobro de iterações.

$\Lambda$ Tabcla 7.35 revela as conclusões que já havíamos tirado com os exemplares gerados aleatoriamente, ou seja, a regra de Dantzig normalizada é inferior à regra usual para alguns problemas. Algumas vezes produz um número muito menor de iterações, mas o tempo computacional é maior.

\subsection{Método DSBU com o Gradiente Bi-Conjugado}

Nesta seção, apresentamos os resultados para o DSBU com o método do gradiente biconjugado (GBC), descrito no capítulo 4, para resolver os sistemas lineares básicos do método dual simplex. A estrutura de dados é dada na seção 6.4 , tanto para armazenar a matriz de restrições (matriz $N$, por linhas), quanto para armazenar a base (matriz $\boldsymbol{B}$. por colunas).

Na Tabela 3.6 apresentamos os resultados para DSBU com o método do gradiente biconjugado, em que reportamos a média do número máximo iteraçôes rcgistradas para este método iterativo. assim como o número de iterações realizadas pelo método dual simplex com busca unidimensional. 
Tabela 7.36-Problemas esparsos

\begin{tabular}{|c|c|c|c|c|c|c|c|c|}
\hline \multicolumn{6}{|c|}{ Característica dos problemas } & \multicolumn{2}{|c|}{$D S B U-G B C$} & \multirow{2}{*}{$\frac{G B C}{\text { it } \max }$} \\
\hline$n b$ & $m 1$ & $n I$ & $c c$ & $\%$ & $m^{\prime} \times n$ & Iteração & tempo & \\
\hline 10 & 5 & 7 & 2 & 13.46 & $50 \times 52$ & 42.2 & 0.00 & 75.3 \\
\hline 20 & 5 & 7 & 2 & 6.86 & $100 \times 102$ & 104.2 & 0.01 & 5.7 \\
\hline 20 & 5 & 12 & 2 & 5.94 & $100 \times 202$ & 15.1 & 0.00 & 15.3 \\
\hline 50 & 2 & 4 & 2 & 3.91 & $100 \times 102$ & 85.6 & 0.00 & 118.6 \\
\hline 100 & 2 & 4 & 2 & 1.98 & $200 \times 202$ & 172.6 & 0.05 & 317.5 \\
\hline 50 & 2 & 3 & 1 & 2.97 & $100 \times 101$ & 79.6 & 0.00 & 63.3 \\
\hline 100 & 1 & 2 & 1 & 1.98 & $100 \times 101$ & 70.8 & 0.00 & 20.7 \\
\hline 200 & 1 & 3 & 1 & 0.74 & $200 \times 401$ & 2.1 & 0.00 & 2.7 \\
\hline 200 & 1 & 2 & 1 & 0.99 & $200 \times 201$ & 142.3 & 0.00 & 32.6 \\
\hline 100 & 2 & 3 & 1 & 1.49 & $200 \times 201$ & 154.3 & 0.04 & 158.3 \\
\hline 20 & 1 & 25 & 5 & 6.17 & $20 \times 405$ & 2.2 & 0.00 & 2.6 \\
\hline 20 & 5 & 25 & 5 & 6.17 & $100 \times 405$ & 12.6 & 0.03 & 15.1 \\
\hline 200 & 1 & 2 & 0 & 0.50 & $200 \times 400$ & 165.5 & 0.09 & 3.0 \\
\hline 200 & 2 & 2 & 0 & 0.50 & $400 \times 400$ & 272.2 & 0.11 & 109.3 \\
\hline 140 & 3 & 3 & 0 & $0 . \overline{71}$ & $420 \times 420$ & 313.5 & 0.12 & 530.4 \\
\hline
\end{tabular}

A Tabela 7.36 mostra que, o método GBC resolveu, em média, os sistemas linearcs básicos em no máximo $1.5 n$ iterações (lembrando que $n$ a dimensão da base no DSBU).

É importante observar que no método GBC não existe preenchimentos (i.e., novos elementos não-nulos) ao contrário da decomposição LU, mesmo com o uso de heurísticas que tentam minimizar este efeito. Notamos também que o tempo de resolução do DSBU com GBC foi baixo.

Comparamos o desempenho do DSBU utilizando a decomposição $L U$ com a heurística de grau mínimo (GM) e o método de atualização de Reid (mclhor combinação, veja seção 7.2) com o DSBU utilizando o método do gradiente bi-conjugado. $\Lambda$ Tabela 3.37 apresenta os resultados.

Tabela 7.37 -Problemas esparsos

\begin{tabular}{|c|c|c|c|c|c|c|c|c|c|}
\hline \multicolumn{6}{|c|}{ Característica dos problemas } & \multicolumn{2}{|c|}{$D S B U-L U$} & \multicolumn{2}{|c|}{$\overline{D S B} U-G B C$} \\
\hline$n b$ & $m I$ & $n 1$ & $c c$ & $\%$ & $m^{\prime} \times n$ & iteração & tempo & iteração & tempo \\
\hline 50 & 2 & 21 & 1 & 2.10 & $100 \times 1001$ & 144.1 & 2.55 & 144.5 & 0.13 \\
\hline 250 & 2 & 5 & 1 & 0.49 & $500 \times 1001$ & 520.7 & 9.4 & 521.2 & 1.10 \\
\hline 250 & 2 & 9 & 1 & 0.45 & $500 \times 2001$ & 613.4 & 113.5 & 612.1 & 1.98 \\
\hline 500 & 2 & 2 & 0 & 0.20 & $1000 \times 1000$ & 698.5 & 13.6 & 697.8 & 1.34 \\
\hline 500 & 2 & 5 & 1 & 0.24 & $1000 \times 2001$ & 1078.5 & 197.4 & 1078.9 & 4.91 \\
\hline
\end{tabular}

Os resultados acima revelam que, para estes problemas de médio porte, o método do gradiente bi-conjugado é muito mais rápido do que a decomposição $\boldsymbol{L} U$ com a heurística GM e o método de atualização de Reid. A maior diferença é observada justamente para os problemas 
maiores, em especial os de dimensão (1000×2001), em que o tempo do DSBU-LU é 40 vezes mais do que o DSBU-GBC.

Vale observar que para os exemplares $(500 \times 1001)$ são feitas 5 decomposições da matriz base e os excmplares de dimensão $(1000 \times 2001)$ são feitas 10 decomposições, já que a frequiencia de refatoração utilizada foi 100 (veja seção 7.2).

A Tabela 7.38 apresenta os resultados da comparação entre o DSBU com o método GBC e o pacote CPLEX para resolver os problemas anteriores.

Tabela 7.38 - Problemas esparsos

\begin{tabular}{|c|c|c|c|c|c|c|c|c|c|}
\hline \multicolumn{6}{|c|}{ Característica dos problemas } & \multicolumn{2}{|c|}{$D S B U-G B C$} & \multicolumn{2}{|c|}{ CPLEX } \\
\hline$\overline{n b}$ & $m I$ & $n 1$ & $c c$ & $\%$ & $m^{\prime} \times n$ & iteração & tempo & iteração & tempo \\
\hline 50 & 2 & 21 & 1 & 2.10 & $100 \times 1001$ & 144.5 & 0.13 & 149.2 & 0.00 \\
\hline 250 & 2 & 5 & 1 & 0.49 & $500 \times 1001$ & 521.2 & $1 . \overline{10}$ & 542.3 & 0.03 \\
\hline 250 & 2 & 9 & 1 & 0.45 & $500 \times 2001$ & 612.1 & 1.98 & 635.7 & 0.05 \\
\hline 500 & 2 & 2 & 0 & 0.20 & $1000 \times 1000$ & 697.8 & 1.34 & 691.6 & 0.13 \\
\hline 500 & 2 & 5 & 1 & 0.24 & $1000 \times 2001$ & 1078.9 & 4.91 & 1094.7 & 0.29 \\
\hline
\end{tabular}

Apesar do DSBU-GBC ser muito superior ao DSBU-LU do ponto de vista do tempo computacional, o tempo do pacote CPLEX é ainda muito menor do que o DSBU-GBC, cerca de 20 a 30 vezes. Contudo, obtivemos uma melhora muito significante no tempo computacional do método dual simplex com busca unidimensional.

De uma maneira geral, o método iterativo do gradiente bi-conjugado se mostrou muito eficiente em resolver os sistemas lineares básicos do método DSBU, principalmente em relação à decomposição LU, mesmo com o uso de heurísticas e métodos de atualização para tratar a esparsidade.

Embora, para um número pequeno de exemplares, o método DSBU com GBC não foi capaz de resolvê-los, na maioria dos testes que realizamos, o método do gradiente bi-conjugado obteve sucesso. Fste fato é interessante, pois as matrizes básicas não têm nenhuma estrutura particular e, apesar do método do gradiente bi-conjugado não ter garantia teórica de convergência para os problemas que utilizamos, o método convergiu na prática.

Portanto, o método iterativo do gradiente bi-conjugado, deve ser alvo de pesquisa futura no contexto do método simplex, assim como outros métodos como, por exemplo, o GMRES (Saad, 2003). 
Capitulo 8 - Conclusões e Trabalhos Futuros

Neste trabalho foi estudada a classe de problemas canalizados ou forma geral (Vanderbei, 1997) de otimização linear que é de grande interesse prático. O problema dual é um problema linear por partes e o método simplex pode ser especializado ao problema dual linear por partes, executando uma busca unidimensional na direção simplex. A esta especialização chamamos de método dual simplex com busca unidimensional (DSBU). Uma abordagem similar foi desenvolvida recentemente por Maros (2003) para variáveis canalizadas.

Aprofundamos a investigação sobre o método dual simplex com busca unidimensional, propondo e analisando variantes para a busca unidimensional linear por partes, exatas ou inexatas, que inclui a adaptação da regra clássica de Armijo e outras específicas à função linear por partes. Concluímos que a busca exata ć superior.

Investigamos também alternativas para o método DSBU, como a direção simplex normalizada ou regra de Dantzig normalizada, que segundo (Forrest e Goldfarb, 1992) fez reduzir significantemente o número de iterações simplex $\mathrm{cm}$ alguns problemas de grande porte. Concluímos que, para a forma geral e para as classes de exemplares gerados e alguns exemplares da Netlib, a normalização da direção simplex pouco influencia no número de iterações e faz aumentar o tempo de resolver os problemas. $\mathrm{O}$ 
comportamento desta regra sobre outras classes de exemplares, grandes e esparsos, ainda merece investigação.

Algumas técnicas para tratar os sistemas lineares básicos foram adaptadas para o método dual simplex com busca unidimensional com o objetivo de explorar a esparsidade, que se faz presente em problemas práticos e de grande porte. Dentre essas técnicas abordamos o estudo da decomposição $L U$ com uso das heurísticas de grau mínimo c Markowitz (1957) e os métodos de alualização da base de Forrest c Tomlin (1977) e Reid (1982). Incluímos a investigação do método itcrativo do gradiente biconjugado. Vale observar que não temos conhecimento na literatura de trabalhos publicados com este tipo de abordagem. Na implementação do método do gradiente bi-conjugado para o método DSBU, surgiu a neccssidade de trabalhar com uma estrutura de dados que atendesse a as operações a serem feitas pelo método GBC e pelas rotinas do método dual simplex com busca unidimensional, de maneira eficiente para problemas esparsos. A estrutura de dados usada foi uma estrutura simples, sugerida recentemente por Maros (2003) na implementação do método simplex. Para isto, foi construído um algoritmo também, que faz a inserção e a remoção de uma coluna na matriz base de maneira eficiente, ainda passível de melhoria. Concluímos que o método do gradiente bi-conjugado acoplado ao método DSBU reduz significantemente o tempo de resolução, quando comparado às tradicionais decomposições LU com técnicas de atualização e heurísticas para manutenção de esparsidade. Os resultados comparados com o pacote CPLEX são promissores, já que em muitos exemplares o número de iterações foi reduzido.

O método dual simplex com busca unidimensional e técnicas de esparsidade, regra de Dantzig normalizada e o procedimento de expansão (anti-degeneração) foram implementados, testados e comparados entre si e com o pacote CPLEX. Pudemos concluir sobre a eficiência de introduzir algum tipo de busca unidimensional no método dual simplex para resolver os problemas canalizados, muito superior do que o método dual simplex usual. Pelos testes realizados com problemas degenerados da Netlib e gerados aleatoriamente, ficou evidente a necessidade também de se utilizar algum procedimento anti-degeneração, os quais influenciam positivamente no fenômeno de estagnação (mudanças de bases, sem alteraçâo do vértice).

Os resultados computacionais mostram que para problemas esparsos o tempo de resolução do CPLEX é bem menor do que o método DSBU, mesmo fazendo mais iterações, evidenciando que estruturas de dados mais adequadas devem ser investigadas. 
Como a regra de Dantzig Normalizada teve seu desempenho equivalente a regra usual para uma classe de problemas e alguns poucos da Netlib, torna-se interessante identificar classes de problemas em que a regra proposta por liorrest e Goldfarb (1992) seja de lato supcrior, como afirmam os autores.

Uma técnica muito eficiente que deve ser investigada, mas que envolve uma implementação bem estruturada e utilizada por muitos pacotes, é o pré-processamento que é aplicado ao problema antes de iniciar o método de resolução. O propósito é reduzir o problema para que possa ser resolvido mais rapidamente, que consiste em fixar variáveis, eliminar restrições redundantes e apertar limitantes, pois desta forma, o problema resultante fica mais fácil de ser resolvido.

É interessante salientar que, embora as matrizes básicas (utilizadas na resolução dos sistemas lineares) não tenham nenhuma estrutura particular, o método GBC obteve sucesso na maioria dos problemas que resolvemos. Portanto, torna-se interessante investir na resolução dos sistemas lineares (do método simplex) com o uso de métodos iterativos como, por exemplo, o GMRES (Saad, 2003) e rever pré-condicionamentos alternativos. 


\section{Referências Bibliográficas}

[1] Adler I., Resende, M., Veiga G. (1986) "An Implementation of Karmarkar's Algorithm for Linear Programming" Report ORC86-8, Operations Research, University of California, Berkeley.

[2] Adler, I., Karp, R., Shamir, R. (1987) "A Simplex Variant Solving an $m \times d$ linear Program in $O(\min (m 2, d 2))$ Expected Number of Steps" Journal of Complexity, 3, 372-387.

[3| Adler, 1. and Megiddo, N. (1985) "A Simplex Algorithm where the Average Number of Steps is Bounded Between Two Quadratic Functions of the Smaller Dimension" Journal of the ACM 32, 871. 895.

[4] Andersen, E. D. and Ye, Y. (1986) "Combining Interior-point and Pivoting Algorithms" Management Science., 42(12):1719-1731.

[5] Anstreicher, K. M. and Terlaky, T. (1994) "A Monotonic Build-up Simplex Algorithm for Linear Programming" Operations, Research 42, 556-561.

[6] Anstreicher, K. M. and Terlaky, T. (1996) "Potential Reduction Methods, in Interior Point Methods in Mathematical Programming" Kluwer Academic Publishers. 
[7] Arenales, M. N. (1984) "Otimizą̧ão Linear: Novos Métodos e Alguns Problemas Particulares" Tese de Doutorado, FEC-UNICAMP.

[8] Avis, D and Chvátal, V. (1978) "Notes on Bland's Pivoting Rule" Mathematical. Programming Study 8, 24-34.

[9] Balbo, A. R. (1991) "Método de Pontos Interiores com Mudança de Escala para Programação Linear" Dissertação de Mestrado, ICMC-USP.

[10] Balinski, M and Tucker, A. (1997) "Duality Theory of Linear Programs Constructive Approach with $\Lambda$ pplications" SIAM Review, 11,3.

[11] Bartels, R. H. and Golub, G. H. (1969) "The Simplex Method of Linear Programming Using LUDecomposition" Communications of the ACM 12,266-268 and 275-278.

[12] Bazaraa, M. S., Jarvis, J. J., Sherali, H. D. (1990) "Linear Programming and Network Flows" $2^{\text {nd }}$ Ed., New York.

[13] Beale, E. (1955) "Cycling in the Dual Simplex Method" Naval Research Logistics Quarterly, 2, 4, 269-275.

[14] Bertsimas, D. and Tsitsiklis, J. N. (1997) "Introduction to Linear Optimization" $2^{\text {nd }}$ Ed., Athena Scientific, Massachusetts.

[15] Bixby, R., Fenclon, M., Gu, Z., Rothberg, E., Wunderling, R. (2000) "MIP: Theory and Practice Closing the Gap" In: Powell, M.J.D., Scholtes, S., eds., Kluwer Publishers, System Modelling and Optimization: Methods, Theory, and Applications, 9-49.

[16] Bixby, R. E. (2001) "Solving Real-World Linear Programs: A Decade and More of Progress", II.OG, Inc and Rice University. http://www.caam.rice.edu/ bixby/default.htm 
[17] Bixby, R. E. (1994) "Progress in Lincar Programming" ORSA J. on Computing, 6, 1, 15-22.

118] Bixby, R. E.., Gregory, J., I ustig, I., Marsten, R., Shano, D. (1992) "Very Large-Scale Linear Programming: $\Lambda$ Case Study in Combining Interior Point and Simplex Methods" Operations Research, $40,885-897$

[19] Bland, R. (3. (1977) "New Finite Pivoting Rules For the Simplex Method" Mathematical Operations Research., 2, 2, 103-107.

[20] Borgwardt, K. H. (1987) "The simplex Method: A Probabilistic Analysis" Springer Verlag, Berlin.

[21] Borgwardt, K. H. (1982) "The Average Number of Pivot Steps Required by the Simplex Method is Polynomial" Zeitschrift fur Operations Research, 26, 155-177.

[22] Cavichia, M. C. and Arenales, M. N. (2000) "Piecewise Linear Programming via Interior Points" Computers \& Operations Research, 27, 13, 1303-1324.

[23] Charnes, $\Lambda$. Cooper, W., Mellon, R. (1952) "Blending Aviation Gasolines-Study in Programming Interdependent Activies in an Integrated Oil Company" Econometrica, 20, 2, 135-159.

[24] Chvátal, V. (1983) "Linear Programming" New York: W. II. Freeman and Company.

[25] Clausen, J. (1979) "A Tutorial Note on the Complexity of the Simplex Algorithm" Technical Report NR79/16, DIKU, Copenhagen University, Copenhagen, Denmark.

[26] Dantzig, G. B. (2002) "Linear Programming" Operations Research, 50, 1, $42-47$

127| Dantzig, G. B. and Wolfc, P. (1961) "The Decomposition Algorithm for Linear Programming" Econometrica, 29, 767-778. 
[28] Dantizg, G. B. (1951) "Maximization of a Linear Function of Variables Subject to Linear Inequalities", in Koopmans, T. C. (ed.), Activity Analysis of Production and Allocation, John Wilcy \& Sons, New York, 339-347.

[29] Dantizg, G. B. (1963) "Linear Programming and Extensions". Princeton University Press, Princeton, $\mathrm{NJ}$.

[30] Dantizg, G. B and Thapa, M. N. (1997) "Linear Programming" Ed. Springer Verlag.

[31] Dantizg, G., Orden, A., Wolfe, P. (1955) "A Generalized Simplex Method for Minimizing a Linear Form under Linear Inequality Constraints" Pacific Journal of Mathematics, 5, 2, 183-195.

[32] Dickson, J. C. and Frederick, F. P. (1960) "A Decision Rule for Improvement Efficiency in Solving Linear Programming Problems with the Simplex Algorithm" Comm. ACM3,509-512.

[33] Duff, I. S., Lirisman, A. M., Reid, J. K. (1986) "Direct Methods for Sparse Matrices" Clarendon Press. Oxford.

[34] Fletcher, R. (1974) "Numerical Analysis Dundee" Springer-Verlag- Lectures Notes in Mathematics, vol. 506 .

[35] Forrest, J. J. and Goldfarb, D. (1992) "Steepest-Fdge Simplex Algorithms for Lincar Programming" Math. Programming 57, 341-374.

[36] Forrest, J. J. and Tomlin, J. A. (1972) "Updated Triangular Factors of the Basis to Maintain Sparsity in the Product Form Soimplex Method" Mathematical Programming. 2, 263-278.

[37] Freund, R. M. and Mizuno, S. (2000) "Interior Point Methods: Current Status and Future Directions" High Performance Optimization, H. Prenk et al. (eds.), Kluwer $\Lambda$ cademic Publishers, 441466 . 
[38] Fukuda, K. and Terlaky, T. (1997) "Criss-cross methos: A fresh view on pivot algorithms" Mathematical Programming Series B, 79, 369-395.

[39] Fukuda, K. and Terlaky, T. (1998) "On the Existence of Short Admissible Pivot Sequences" Technical Report 98-48, Delft University of Technology, Delft, The Netherlands.

[40] Gass S. 1. (1985) "Linear Programming:Methods and Applications" 5th ed. New York, McGrawHill Book Company.

[41] Gass, S. I. (1993) "Encounters with Degeneracy: a Personal Vicw" Annals of Operations Research, 47, $335-42$.

[42] Gass, S. I. and Vinjamuri, S. (2004) "Cycling in linear Programming Problems" Computers and Operations Research, 31, 303-311.

[43] Gay, D. M. (1985) "Eletronic Mail Distribution of I.inear Programming Test Problems", Mathematical Programming Society COAL Newsletter, December.

[44] Gill, P. E., Murray, W., Saunders, M.A., Wright, M. H. (1989) " $\Lambda$ Practical Anti-cycling Procedure for Linearly Constrained Optimization" Math. Programming, 45, 437-474.

[45] Gilmore, P. and Gomory, R. (1965) "Multistage Cutting Stock Problems of Two and More Dimensions" Operations Research, 14, 94-120.

[46] Goldfarb, D. (1983) "A Numerically Stable Dual Method for Solving Strictly Convex Quadratic Programs" Mathematical Programming, 27, 1-33.

[47] Goldfarb, D. and Reid, J. K. (1977) "A Practicable Steepest-Edge Simplex Algorithm" Math. Programming, 12, 361-371. 
[48] Goldfarb, D. and Sit, W. Y. (1979) "Worst Case Behavior of the Steepest Edge Simplex Method" Discrete Appl. Math., 277-285.

[49] Golub, G. H. and Loan, C. V. (1989) "Matrix Computations" 2nd ed. Baltimore: Jonhs 1 lopkins Universaty Press.

[50] Gonzaga, C. (1992) "Path Following Methods for Linear Programming" SIAM Review, 34,

$2,167-227$.

[51] Harris, P.M. (1973) "Pivot Selection Method of the Devex L.P Code" Mathematical Programming $5,1-28$.

[52] Hoffman, A. J. (1953) "Cycling in the Simplex Algorithm" Washington, DC, National Bureau of Standards.

153] Kamarkar, N. (1984) "A New Polynomial-Time Algorithm for Linear Programming" Combinatorica, 4, 373-395.

[54] Khachiyan, L. G. (1979) "A Polynomial Algorithm for Linear Programming" Dokla. Akad. Nauk. USSR, 244, 5, 1093-96.

[55] Klec, V. and Minty, G. J. (1972) "How Good is the Simplex Algorithm?" in Shish, Proc. 3rd Sympos. Inequalities, AP, New York, 159-175.

[56] Kuhn, H. W. and Quandt, R. E. (1963) "An Experimental Study of the Simplex Method" in Metropolis et al. (Eds.), Proc. 15th Sympos. Appl. Math., Amer. Math. Soc., Providence, R.1., 107-124.

[57] Lanczos, C. (1952) "Solution of Systems of Linear Equations by Minimized Iterations" Journal of Research of the National Bureau of Standards, 49, 33-52. 
[58] Lemke, C. E. (1954) "The Dual Method of Solving the Linear Programming Problem" Naval Research Logistics Quarterly, 1, 36-47.

[59] Luenberger, D.G. (1984) "Linear and Nonlinear Programming" Addison-Wesley.

[60] Luh, H and Tsaih, R. (2002) "An Efficient Search Direction for linear Programming Problems", Computers \& Operations Research, 29, 195-203.

[61] Markowitz, H. M. (1957) "The Elimination Form of the Inverse and its $\Lambda$ pplications to Linear Programming" Management Science, 3, 255-269.

[62] Maros, I. (2003") "A Generalized Dual Phase-2 Simplex Algorithm" European Journal Operational Research, 149, 1-16.

[63] Maros, I. (2003) "Computational Techniques of the Simplex Method" Kluwer Academic Publishers.

[64] Maros, I. and Mitra, G. (1996) "Simplex Algorithms" In. J. Beasley, editor, Advances in Linear and Integer Programming. Oxford University Press, Oxford, England.

[65] Marshall, K. and Suurballe, J. (1969) "A note on Cycling in the Simplex Method" Naval Research Logistics Quarterly, 16, 1, 121-127.

[66] Murty, K. G. (1983) "Linear Programming”, John Wiley \& Sons.

[67] Nering, E. and Tucker, A. (1993) "Linear Programs and Related Problems" Boston, MA: $\Lambda$ cademic Press.

[68] Oishi, C. M. (2004) "Análise e Implementação de Métodos Implícitos no Sistema FreeFlow2D" Disscrtação de Mestrado, ICMC-USP. 
[69] Paparrizos, K. (1996) "Exterior Point Simplex Algorithm: Simple and short proof of correctness" Proceedings of SYMOPIS'96, 13-18.

[70] Paparrizos, K. (1989) "Pivoting Rules Directing the Simplex Method Through all Feasible Vertices of Klec-Minty Lxamples" Operations Research, 26, 77-95.

[71] Pinto, M. J. (1999) "O Prohlema de Corte de Estoque Inteiro" Dissertação de Mestrado, ICMCUSP.

[72] Press, W. H., Flannery, B. P., Teukolsky, S. A., Vetterling, W. T. (1992) "Numerical Recipes in C: The Art of Scientific Computing" 2nd ed. Cambridge-University Press.

[73] Quarteroni, A., Sacco, R., Saleri, F. (2000) "Numerical Mathematics" Springer.

[74] Reid, J. K. (1982) "A Sparsity-Exploiting Variant of the Bartels-Golub Decomposition for Linear Programming Bases", Mathematical Programming, 24, 55-69.

[75] Roos, C. and Terlaky, T. (1996) "Advances in Linear Optimization" Report Nr. 96-118, Faculty of" Technical Mathematics and Informatics, Delf, Netherland.

[76] Ryan, D. and Osborne, M. (1988) "On the Solution of Highly Degenerate Linear Programmes" Mathematical Programming, 41, 385-392.

[77] Saad, Y. (2003) "Iterative Methods for Sparse Linear System." Second Edition, Society lor Industrial and $A$ pplied Mathematics, Philadelphia, PA, USA.

[78] Saad, Y, and Schultz, M. (1986) "GMRES: A Generalized Minimal Residual Algorithm for Solving NonSymmetric Linear Systems" SIAM J. Sci. Stat. Comput., 7, 3, 856-869.

[79] Shamir, R. (1987) "The Efficiency of the Simplex Method: A Survey" Management Science, 3, $301-334$. 
[80] Sierksma, G. (1996) "Linear and Integer Programming" 2nd ed. New York: Marcel Dekker, Inc.

[81] Silva, C. T. I. (2002) "Problemas de Otimização Linear Canalizados e Esparsos" Dissertação de Mestrado, ICMC-USP.

[82] Smale, S. (1983) "On the Average Speed of the Simplex Method in Linear Programming" Mathematical Programming, 27, 241-262.

[83] Solow, D. (1984) "Linear Programming: an introduction to finite improvement algorithms" Amsterdan: North-Holland.

[84] Sousa, R. S. (2000) "Estudos em Otimização Linear" Dissertação de Mestrado, ICMC-USP.

[85] Stern, J. M. (1994) "Esparsidade, Estrutura, Estabilidade e Escalonamento em Álgebra Linear Computacional", IX Escola de Computação, Recife.

[86] Suhl, U. H. and Suhl L. M. (1990) "Computing Sparse (LU) Factorizations for Large-Scale Linear Programming" ORSA Journal on Computing, 2, 325-335.

[87] Terlaky, T. (2000) "Linear Programming. Simplex-Type Algorithms" Department of Computing and Software, McMaster University, I Iamilton, ON, Canada. http://www.cas.mcmaster.ca/ terlaky

[88] Terlaky, T. (1987) "A Finite Criss-Cross Method for Oriented Matroids" J. Combin. Theory (Ser. B) $42,319-327$.

[89] 'lerlaky, T. (1985) "Methods of 'Hungarian Type' for Solving Linear Programming Problems" Alkalmazolt Matematikai Lapok, 11, 1-14.

[90] Terlaky, T. and Zhang, S. (1993) "Pivot Rules for Linear Programming: A Survey on Recent Theorical Developments" Annals of Operation Research 46, 203-233. 
[91] Todd, M. (2001) "The Many Facets of Linear Programming" Mathematical Programming Ser. B.

[92] Todd, M. (1997) "Potential-reduction Methods in Mathematical Programming" Mathematical Programming, 76, 3-45.

[93] Vanderbei, R. J. (1997) "Linear Programming: Foundations and Extensions", Kluwer Academic Publishers.

[94] Van der Vorst, H. A. (1992) "Bi-cgstab: A Fast and Smoothly Converging Variant of Bi-cg for the Solution of Nonsymmetric Linear Systems" SIAM Journal of Science and Statistic Compuing, 13. $2,631-644$.

[95] Vieira, H. and Lins, M. P. (2005) "An Improved Initial Basis for the Simplex Algorithm" Computers \& Operations Research, 32, 1983-1993.

[96] Wolfe, P. (1963) "A Technique for Resolving Degeneracy in Linear Programming" SIAM Journal of Applied Mathematics, 11, 205-211.

[97] Wolfe, P. and Cutlcr, L. (1963) "Experiments in Linear Programming" in Graves and Wolfe (Eds.) Recent Advances in Mathematical Programming, McGraw Hill, New York, 177-200.

[98] Yudin, D. B. and Golshtein, E. G. (1965) "Linear Programming" Israel Program of Scientific Translations, Jerusalem.

[99] Zhang, S. (1999) "A New Variant of Criss-Cross Pivot Algorithm for Linear Programming" European Journal of Operations Research, 116,3, 607-614.

[100] Zionts, S. (1969) "The Criss-Cross Method for Solving Linear Programming Problems" Manag. $S_{\mathcal{C} i,}, 15,426-445$. 


\section{Apêndice A}

\section{A.1. Os Formatos de Arquivo LP e MPS}

O formato do arquivo de entrada lp (Linear Programming) é o formato em que geralmente surgem os modelos de problemas de otimização linear e são apresentados numa forma orientada por linhas (equações), veja o exemplo dado pela Figura A.1.

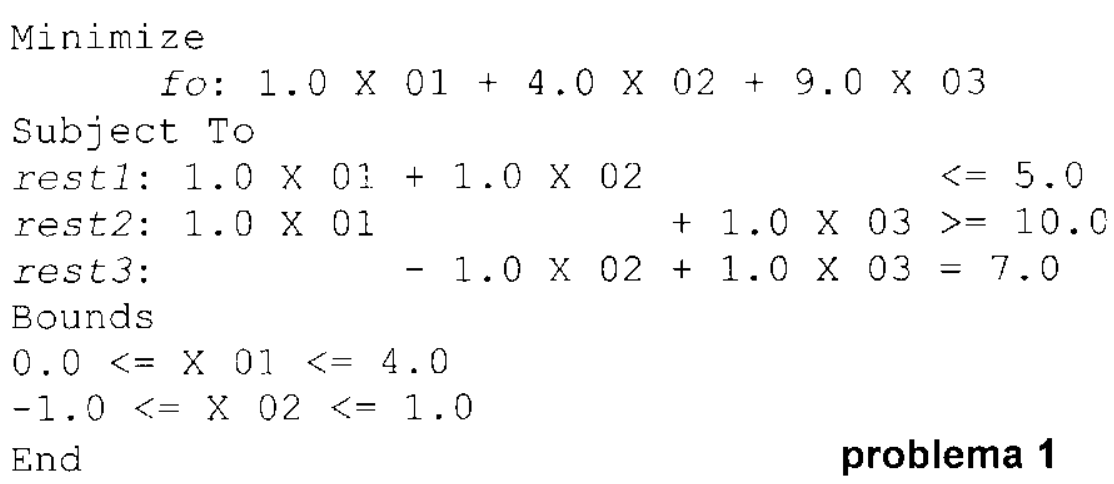

Figura A.1-Problema 1 no formato $1 \mathrm{p}$

Já o formato do arquivo de entrada mps (Mathematical Programming System) foi originalmente introduzido pela IBM para expressar problemas lineares inteiros em uma forma padrão. 
É um tipo de formato fixo para as colunas. Descreveremos uma pequena introdução para o formato mps de problemas de otimização linear e os exemplos. Para mais informações, veja Nazareth (1987)

O formato mps é a forma de apresentar os dados do problema orientado por coluna, contrário ao formato $1 p$. No formato mps não define para a funçào objetivo a maximização (MAX) ou minimização (MIN).

Dependendo do pacote de otimização utiliza-se MAX ou MIN , então deve se tomar o cuidado de saber qual objetivo.

Na Figura A.2 apresentamos o problema 1 (Figura A.1) no formato mps.

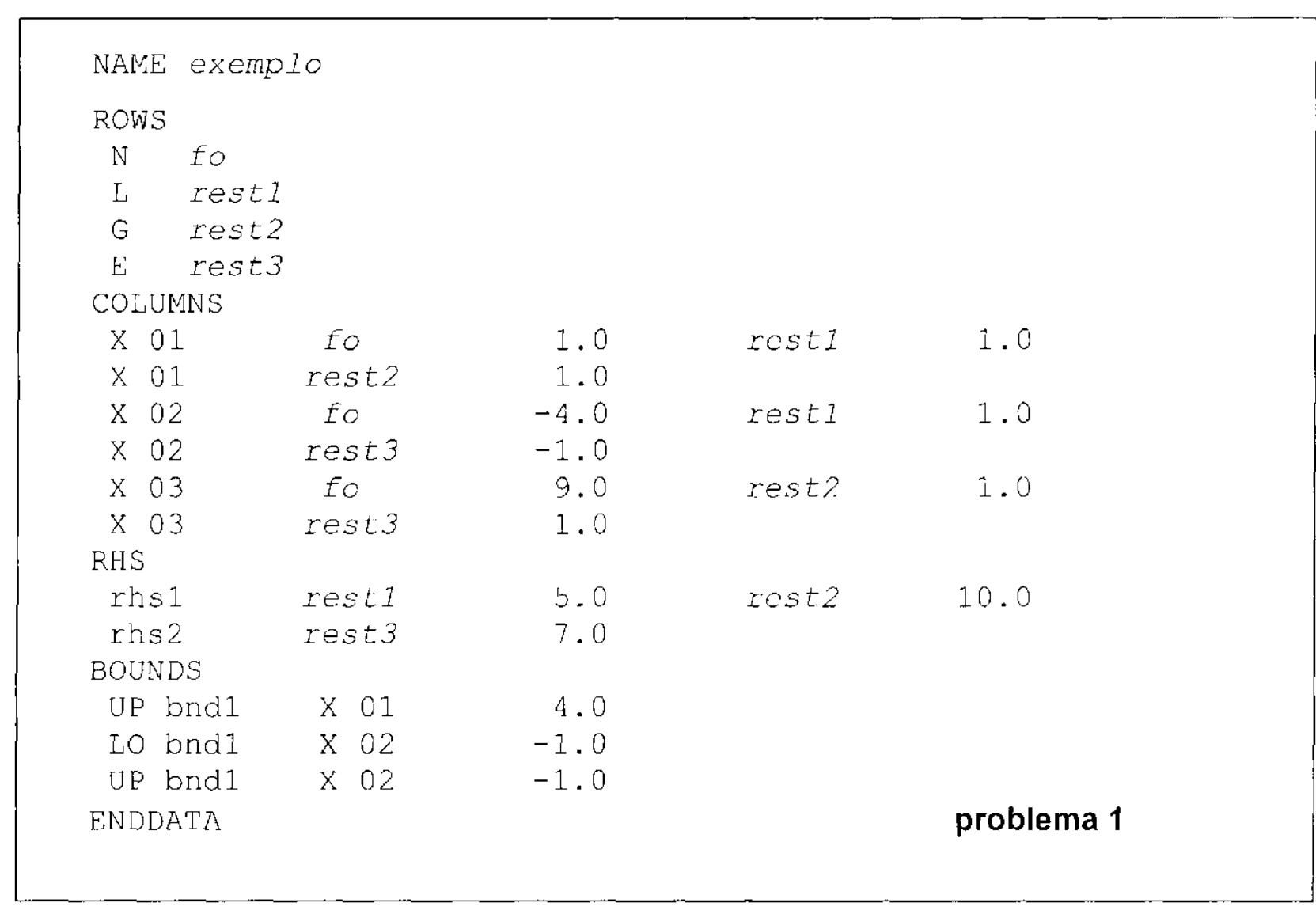

Figura A.2 $\cdots$ O problema 1 no formato mps

Assim, de uma maneira geral, na Figura A.3, apresentamos como os dados de um problema em formato mps devem estar dispostos. 


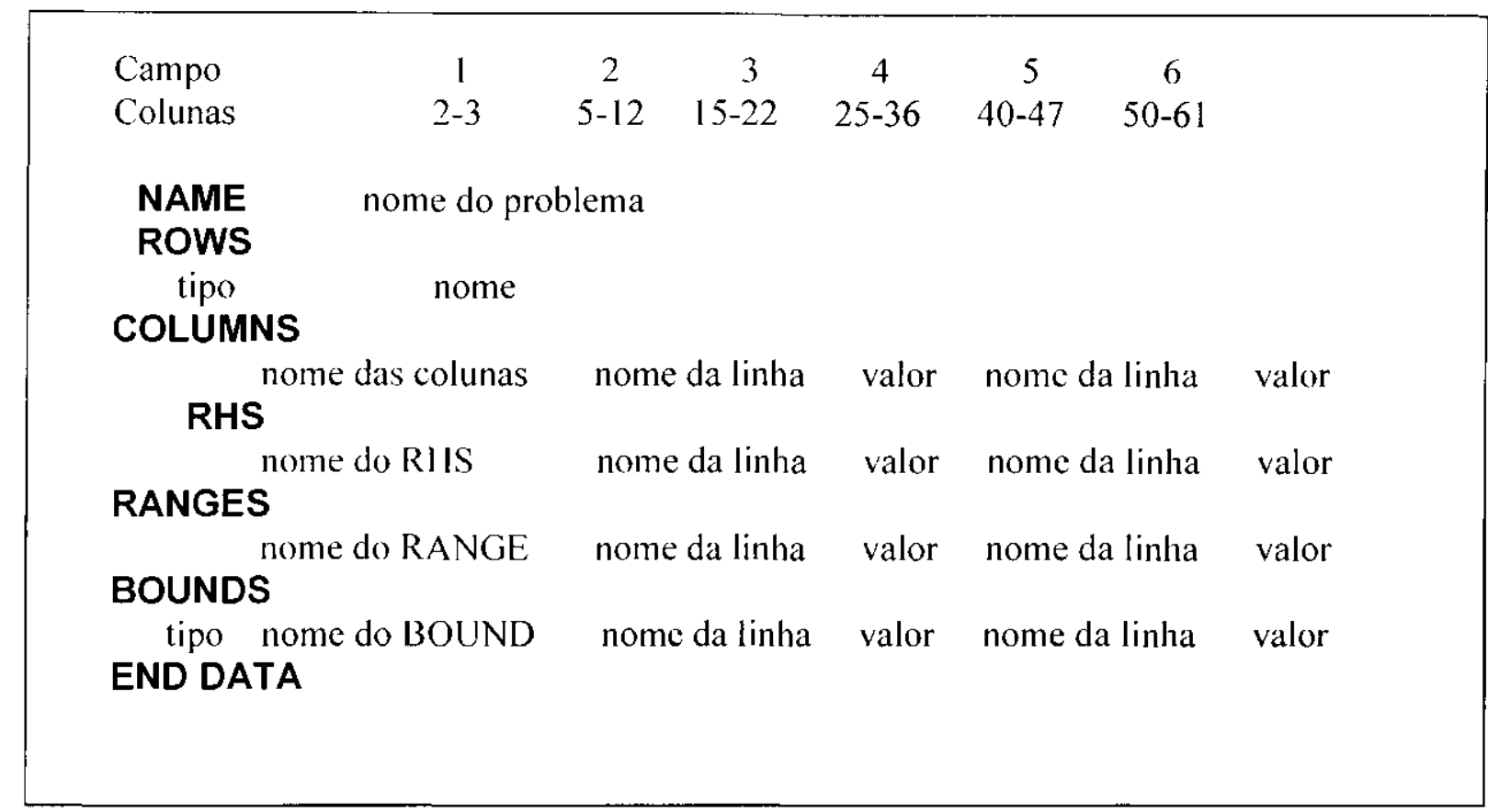

Figura A.3 - Modelo para os dados em formato mps

Na seção ROWS, cada linha deve ter um nome e o tipo de restrição, que é dado pela Tabela A.I.

Tubela A.1 - Tipo de restrição

\begin{tabular}{|c|c|}
\hline tipo & significado \\
\hline$E$ & Igualdade \\
\hline$L$ & Menor ou igual \\
\hline$G$ & Maior ou igual \\
\hline$N$ & Sem restrição \\
\hline
\end{tabular}

Na seção COLUMNS, os nomes das variáveis são definidos ao longo dos coeficientes da função objetivo e de todos os elementos diferentes de zero na matriz de restrições.

A seção RANGES é para as restrições da forma: $d_{i} \leq$ restrição $i \leq \boldsymbol{e}_{i}$. O RANGE da restrição é $r=\boldsymbol{e}-\boldsymbol{d}$. O valor de $\boldsymbol{r}$ é especificado na seção RANGES, c o valor de $\boldsymbol{d}$ ou $\boldsymbol{e}$ é especificado na seção RHS.

Se vetor o $b$ é colocado na seção RHS, e $\boldsymbol{r}$ é o valor 'colocado' na seção RANGES, então os componentes dos vetores $d$ e $e$ são determinados pela Tabela A.2. 
Tabela A.2 - Tipo de restrição e valores para os limitantes

\begin{tabular}{|c|c|c|c|}
\hline Tipo da restricăo & Sinal de $r_{1}$ & Valor de $d_{i}$ & Valor de $e_{i}$ \\
\hline$G$ & + ou & $b_{i}$ & $b_{i}+r_{i} \mid$ \\
\hline$L$ & $+o u-$ & $b_{i}-\mid r_{i}$ & $b_{i}$ \\
\hline$E$ & + & $b_{i}$ & $b_{i}{ }^{\prime} r_{i} \mid$ \\
\hline$E$ & - & $b_{i}-\mid r_{i}$ & $b_{i}$ \\
\hline
\end{tabular}

A seção BOUNDS, os limitantes nas variáveis são especificados. Quando os limitantes não são identificados, o valor usual é para as variáveis que são maiores do que zero, ou seja, $0 \leq x \leq+\infty$. A Tabela $A .3$ apresenta a legenda para o tipo de restrição.

Tabela A.3 - Tipo de restrição e o significado

\begin{tabular}{|c|c|c|}
\hline tipo & significado & relaçá \\
\hline$L O$ & limitante inferior & $b \leq \boldsymbol{x}$ \\
\hline$G P$ & limitante superior & $\boldsymbol{x} \geq \boldsymbol{b}$ \\
\hline$F X$ & variável fixa & $\boldsymbol{x}=\boldsymbol{b}$ \\
\hline$F R$ & variável livre & $-\infty \leq \boldsymbol{x} \leq+\infty$ \\
\hline$M I$ & limite inferior $-\infty$ & $-\infty \leq \boldsymbol{x}$ \\
\hline$B V$ & variável binária & $\boldsymbol{x}=\boldsymbol{0}$ ou $\boldsymbol{x}=1$ \\
\hline
\end{tabular}

Na seção RANGES e BOUNDS os campos 5 e 6 são opcionais. Veja o exemplo abaixo no formato lp em que apresentamos somente uma restrição com duas variáveis e seguir as duas possibilidades de representá-la no formato mps.

$$
\text { rest } 1: 2 x_{1}+3 x_{2}<=6
$$

Então as duas possibilidades de entrar com a variável $x_{1}$ na seção COLUMNS são dadas pela Figura A.4. 


\begin{tabular}{rrrrrr}
\hline (Ficld) & 2 & 3 & 4 & 5 & 6 \\
\hline possibilidadel & $x_{1}$ & restl & 2.0 & rest2 & 5.0 \\
\hline \multirow{2}{*}{ possibilidade2 } & $x_{1}$ & rest & 2.0 & & \\
& $x_{1}$ & rest2 & 5.0 & & \\
\hline
\end{tabular}

lïgura $A .4 \cdots A$ restrição restl representada de duas maneiras

Para deixar bem claro os formatos, apresentamos na Figura A.5 o mesmo exemplo da Figura A.1 alterando-se os nomes das variáveis e restrições nos formatos lp e mps.

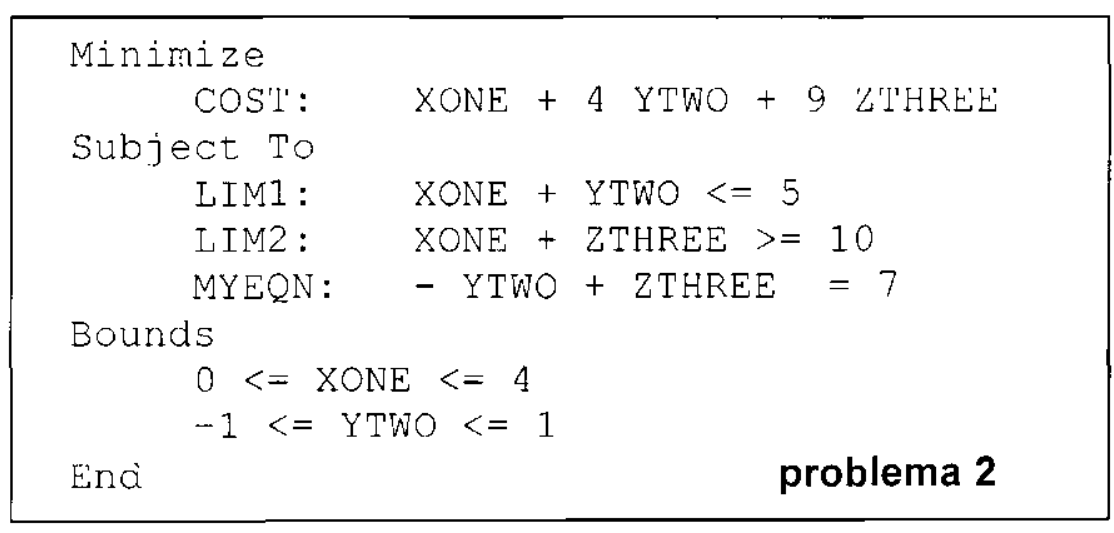

Figura A.5 - Problema 2 no formato lp

Logo o problema da Figura A.5 pode ser apresentado no formato mps dado pela Figura A.6 a seguir. 


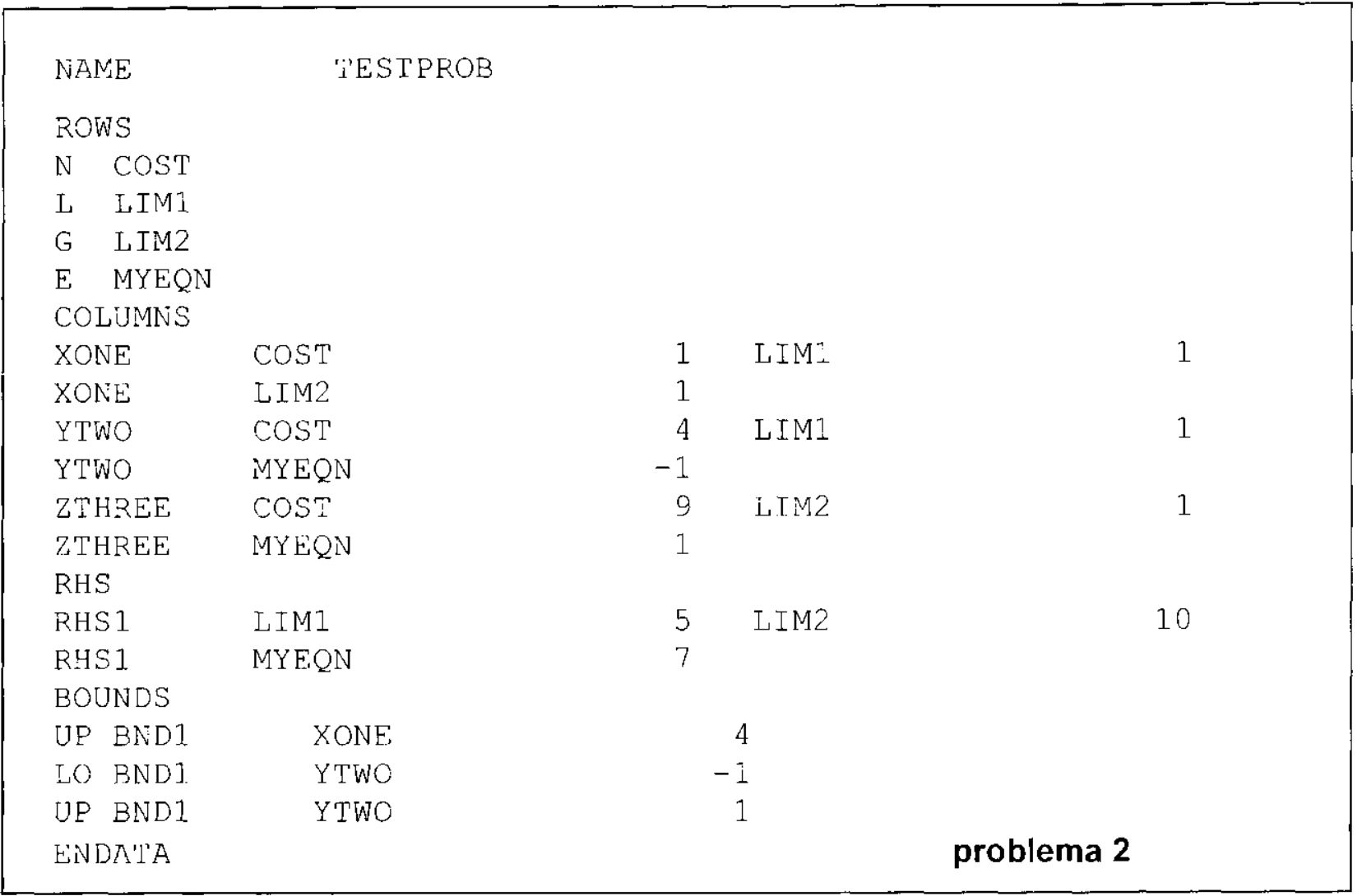

Figura A.6 - O problema 2 no formato mps

Existem na literatura diversos problemas de otimização linear que surgem de aplicações práticas $c$ alguns desses problemas encontram-se na biblioteca Netlib (Gay, 1985) (http:/www.netlib.org) que contém programas, documentos e bancos de dados de interesse para a computação numérica e outras comunidades, cujo domínio é público. A biblioteca é mantida pela AT\&T Bell Laboratories, a Universidade de Tennessee c Oak Ridge National Laboratory.

Os problemas de otimização lincar da Netlib, estão no formato mps compactado. Portanto, é preciso descompactá-los para obter o formato orientado por coluna, descrito anteriormente. A própria Netlib disponibiliza um arquivo executável para realizar tal tarefa. O procedimento para obtermos o problema no formato .lp é bem simples. Uma vez descompactado o problema, este é lido pelo CPLEX e gravado num arquivo com extensão .Ip. Porém, para cada problema as variávcis são formadas por conjunto de caracteres que variam muito em tamanho e na natureza (como combinações Ictras c/ou números). Infelizmente, este procedimento tem uma saída não uniforme, em que 
conseguimos recuperar os dados originais somente para poucos problemas (afiro, adlittle, kb2, sc50a e sc50b).

\section{A.2. Ilog CPLEX Optimizer e XPRESS Optimizer}

Existem diversos pacotes comerciais de otimização linear e relatamos aqui sucintamente dois bem difundidos que fizcram parte dos nossos experimentos computacionais, que são o CPLEX c o XPRLSS (licença para estudante).

XPRESS Optimizer

O pacote XPRESS-Optimizer contém o método simplex que inclui métodos primais e duais para resolver os problemas e um método de pontos interiores. O pacote XPRESS-MP trata de maneira eficiente matrizes esparsas, que permite resolver problemas grandes $\mathfrak{c}$ também é notável por sua habilidade para resolver problemas numericamente instáveis (Terlaky, 1997).

\section{ILOG CPLEX Optimizer}

O pacote CPLEX Simplex Optimizers tem uma implementação robusta dos métodos simplex primais e duais. Todos os algoritmos são integrados com pré-processamento que reduz o tamanho do problema. O método Barreira do CPLEX resolve problemas lineares e quadráticos e está baseado em um método tipo preditor-corretor primal dual. É muito eficiente na exploração de matrizes esparsas.

Mais informações podem ser obtidas nos sites relacionados abaixo.

- II OG CPIEX http://www.cplex.com

- XPRESS-MP http://www.dashoptimization.com

\section{A.3. Parâmetros do Pacote CPLEX 7.5}

Apresentamos aqui, informações sobre alguns parâmetros do CPLEX 7.5. que foram obtidas do manual.

CPLEX's Dual Simplex Optimizer

Recentes avanços computacionais no método dual simplex o têm colocado como a primcira escolha para resolver um problema de otimização linear. Isto é o caso especialmente para problemas 
primal degenerados com pouca variação no termo independente, mas com significante variação no vetor de custos.

\section{CPLEX's Primal Simplex Optimizer}

$\Lambda$ pesar dos recentes avanços no método dual simplex, o método primal simplex terá um desempenho melhor em problemas em que o número de variáveis excede o número de restrições significantemente, ou em problemas que têm pouca variação no vetor de custos. Como poucos problemas têm desempenhos numéricos deficientes em ambas as formas primal $\mathfrak{c}$ dual, o método primal simplex deve ser usado quando ocorre dificuldade numérica no uso do método dual simplex. Pré-processamento

O pré-processamento de um problema é realizado por duas ferramentas bem poderosas: presolve e aggregator que simplificam restrições eliminam variáveis e conseqüentemente reduzem o tamanho do problema.

O presolve tenta reduzir o tamanho de um problema, identificando restrições redundantes e variáveis fixas. O aggregator tenta eliminar linhas e variáveis por substituição. Ocasionalmente as substituições realizadas pelo aggregator aumentam a densidade da matriz. Mas existe um parâmetro que limita as substituições para minimizar o surgimento de um novo elemento diferente de zero.

Refatoração

O CPLEX determina automaticamente a freqüencia em que a base de um problema é refatorada a fim de maximizar a velocidade de cada iteração. Em problemas muito grandes o CPLFX refatora a matriz base raramente. Uma maneira de conservar memória é aumentar a lreçüência de refatoração. Então, se existe pouca memória disponível o CPLEX automaticamente irá diminuir o intervalo de refatoração para usar menos memória em cada iteração.

Degeneração

Problemas altamente degenerados tendem a provocar o fenômeno de estagnação. Quando ocorre esta situação usando o método primal, o pacote CPLEX perturba automaticamente os limitantes das variáveis e quando se usa o método dual, ele perturba a função objetivo. A perturbação cria um problema próximo do original, menos degencrado. Resolvido o problema com as perturbações, o CPIEX remove-as e encontra a solução do problema original. 


\section{Apêndice B}

\section{B.1. O Método Primal Simplex}

Considere o problema (primal) padrão de otimização linear:

$$
\begin{aligned}
& \text { Minimizar } f(x)=c^{\mathrm{T}} x \\
& \text { Sujeito a: } \boldsymbol{A x}=\boldsymbol{b} \\
& x \geq 0,
\end{aligned}
$$

em que $A$ é uma matriz $m \times n$. Suponha que o posto $(A)=m$. Esta hipótese elimina restrições redundantes e casos triviais de inconsistência do sistema $\boldsymbol{A} \boldsymbol{x}=\boldsymbol{b}$. Considere uma partição nas colunas da matriz $\boldsymbol{A}$, chamada partição básica, definida por $\boldsymbol{A}=[\boldsymbol{B}, N]$, cm que $\boldsymbol{B}$ é uma matriz $m \times m$ nãosingular chamada matriz básica e $N$ é uma matriz $m \times(n-m)$ chamada matriz não-básica. Esta partição é possível, se e somente se, a hipótese posto $(\boldsymbol{A})=m$ é verificada. A mesma partição é feita no vetor das variáveis $\boldsymbol{x}=\left[\begin{array}{l}\boldsymbol{x}_{B} \\ \boldsymbol{x}_{N}\end{array}\right]$ de modo que o sistema $\boldsymbol{A x}=\boldsymbol{b}$, pode ser rescrito por: $\boldsymbol{x}_{i 3}=\boldsymbol{B}^{-1} \boldsymbol{b}-\boldsymbol{B}^{-1} \boldsymbol{N} \boldsymbol{x}_{N}$. As variáveis em $\boldsymbol{x}_{B}$ são chamadas variáveis básicas e as variáveis em $\boldsymbol{x}_{N}$, variáveis não-basicas. 
Definição 1. A solução particular $x$ obtida por: $x_{B}=B^{-1} b$ e $x_{N}=0$, é chamada solução básica. Se $\boldsymbol{x}_{B}=\boldsymbol{B}^{-1} b \geq 0$, então a solução básica é primal factivel c dizemos que a partição básica é primal factivel. Se $\boldsymbol{x}_{B}=\boldsymbol{B}^{-1} \boldsymbol{b}>\mathbf{0}$ a solução é dita não degenerada.

Teorema 1. Uma solução básica factível corresponde a um vértice da região factível.

Teorema 2. Se existe solução ótima, então existe um vértice ótimo (solução básica ótima).

Suponha que fosse feita uma lista com todas as submatrizes $\boldsymbol{B}_{m \times \times m}$ de $\boldsymbol{A}$. Algumas dessas submatrizes produziriam soluções básicas factíveis, enquanto outras não, a saber, as que são singulares e aquelas associadas a soluções básicas infactíveis. Portanto, o número de soluções básicas factíveis é menor ou igual a:

$$
\left(\begin{array}{l}
n \\
m
\end{array}\right)=\frac{n !}{(n-m) ! m !} \text {. }
$$

É interessante observar que, embora o teorema 2 nos diga que basta procurar a solução ótima entre os vértices da região de factibilidade, esta procura nos remete a um conjunto de cardinalidade não-polinomial em termos dos dados do problema, podendo introduzir uma complexidade que o problema original não tem.

Definição 2. Chamamos o vetor $\pi \in R^{m}$, dado por:

$$
\pi^{\mathrm{T}}-c_{B}^{\mathrm{T}} B^{-1}
$$

de vetor multiplicador simplex.

Teorema 3. Se a partição básica $\boldsymbol{A}=[\boldsymbol{B}, N]$ é primal factível e $c_{j}-\pi^{T} \boldsymbol{a}_{j} \geq 0, j=1, \ldots, n$, então a solução básica associada (definição 1) é ótima.

Observe que, pela definição de $\pi, c_{i}-\pi^{\mathrm{T}} \boldsymbol{a}_{j}=0$ para $\boldsymbol{a}_{i} \in \boldsymbol{B}$.

Considere uma solução básica primal factível não degenerada $\mathbf{x}^{0}-\left(\begin{array}{c}\boldsymbol{x}_{B}^{0} \\ \boldsymbol{x}_{N}^{0}\end{array}\right)=\left(\begin{array}{c}\boldsymbol{B}^{-1} \boldsymbol{b} \\ \mathbf{0}\end{array}\right)$ cujo o valor da função objetivo $f\left(x^{0}\right)$ é dado por: 


$$
f\left(\boldsymbol{x}^{\mathrm{O}}\right)=\left(\boldsymbol{c}_{B}^{\mathrm{T}}, c_{N}^{\mathrm{T}}\right)\left(\begin{array}{c}
\boldsymbol{B}^{-1} \boldsymbol{b} \\
\mathbf{0}
\end{array}\right)=c_{B}^{\mathrm{T}} \boldsymbol{B}^{-1} \boldsymbol{b}=\boldsymbol{c}_{B}^{\mathrm{T}} \boldsymbol{x}_{B}^{0} .
$$

O sistema $A \boldsymbol{x}=\boldsymbol{b}$ pode ser reescrito por:

$$
\begin{aligned}
& \boldsymbol{x}_{B}=\boldsymbol{B}^{-1} \boldsymbol{b}-\boldsymbol{B}^{-1} \boldsymbol{N} \boldsymbol{x}_{N} \\
& \boldsymbol{x}_{B}=\boldsymbol{B}^{-1} \boldsymbol{b}-\sum_{j \subset N} \boldsymbol{B}^{-1} \boldsymbol{a}_{j} x_{j} \\
& \boldsymbol{x}_{B}=\mathbf{x}_{\mathrm{B}}^{0}+\sum_{j \in N} \boldsymbol{a}_{j} x_{j}
\end{aligned}
$$

em que $N$ é o conjunto dos índices das colunas (ou variáveis) não básicas e $\boldsymbol{\alpha}_{j}=-\boldsymbol{B}^{-1} \boldsymbol{a}_{j}$.

A solução geral do sistema $\boldsymbol{A} \boldsymbol{x}=\boldsymbol{b}$ dada por (B.2) permite escrever também a função objetivo em termos das variávcis não básicas, ou scja,

$$
\begin{aligned}
& f(x)=c^{\mathrm{T}} x \\
& f(x)=c_{B}^{\mathrm{T}} x_{B}+c_{N}^{\mathrm{T}} x_{N} \\
& f(x)=c_{B}^{T}\left(x_{B}^{0}+\sum_{j \in N} \alpha_{j} x_{j}\right)+\sum_{j \in N} c_{j} x_{j} \\
& f(x)=f\left(x^{0}\right)+\sum_{j \in N}\left(c_{j}-\pi^{\mathrm{T}} a_{j}\right) x_{j},
\end{aligned}
$$

que mostra como a função objetivo, restrita ao sistema $A \boldsymbol{x}=\boldsymbol{b}$, se altera quando fazemos perturbações na solução básica factível, por alterar os valores das variáveis não-básicas.

O valor $\left(c_{j}-\pi^{\top} a_{j}\right)$ é referido algumas vezes como custo reduzido ou custo relativo, já que ele é o cocficiente da variável não básica $j$ no espaço reduzido, ou no espaço relativo às variáveis não básicas.

Note que o problema (B.1) pode ser reescrito como: 


$$
\begin{gathered}
\text { Minimizar } f(x)=f\left(x^{0}\right)+\sum_{j \in N}\left(c_{j}-\pi a_{j}\right) x_{j} \\
\text { Sujeito a: }-\sum_{j \in N} \alpha_{j} x_{j}+x_{B}=x_{B}^{0} \\
x_{j} \geq 0, j \in N, \text { e } x_{B} \geq \mathbf{0}
\end{gathered}
$$

As variáveis $\boldsymbol{x}_{B}$ simplesmente fazem o papel das variáveis de folga na equação (B.3). $\Lambda$ ssim, podemos equivalentemente escrever o problema (B.I) no espaço das variáveis não básicas:

$$
\begin{gathered}
\text { Minimizar } f(x)=f\left(x^{0}\right)+\sum_{j \in N}\left(c_{j}-\pi^{\mathrm{T}} a_{j}\right) x_{j} \\
\text { Sujcito a: }-\sum_{j \in N} \alpha_{j} x_{j} \leq \mathbf{x}_{\mathrm{13}}^{0} \\
x_{j} \geq 0, j \in N
\end{gathered}
$$

Como o número de variáveis não básicas é $p=(n-m)$ podemos representar o programa linear num espaço $p$-dimensional. Isto é esperado, já que existem $p$ variáveis independentes ou $p$ graus de liberdade no sistema $\boldsymbol{A x}=\boldsymbol{b}$.

Definição 3. Chamamos de Estratégia Primal Simplex a seguinte perturbação da solução básica inicial:

- Faça $x_{q}-\varepsilon \geq 0$, para algum $q \in N$ tal que $c_{q}-\pi^{\top} \boldsymbol{a}_{q}<0$,

- Faça $x_{j}=0$ para todo $j \neq q, j \in N$.

A estratégia simplex produz uma nova solução dada por:

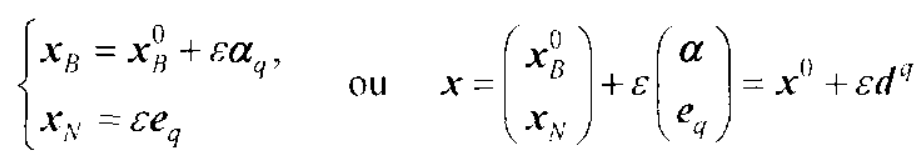

em que $e_{q}=(0, \ldots, 1, \ldots, 0)^{\mathrm{r}} \in R^{n-m}$, com 1 na $q$-ésima componente. 
Note que a direção $d^{q} \in R^{n}$, dada por $d^{q}=\left(d_{B}^{q}, d_{N}^{q}\right)^{\mathrm{T}}=\left(\alpha, e_{q}\right)^{\mathrm{T}}$, define uma perturbação da solução básica e é chamada direção simplex. Se a solução básica for não degenerada, ou seja, $\boldsymbol{x}_{B}^{0}>\mathbf{0}$, de (B.4) segue que $d^{q}$ é uma direção factível em $x^{0}$, uma vez que produzirá soluções factíveis para $\varepsilon>$ 0 c pequeno. Note ainda, $c^{\mathrm{T}} d^{4}=c_{q}-\pi^{\mathrm{T}} a_{q}$.

Usando (B.4) podemos escrever o valor da função objetivo para a solução perturbada por:

$$
f(x)=c^{\mathrm{T}}\left(x^{0}+\varepsilon d^{g}\right)=f\left(x^{0}\right)+\varepsilon c^{\mathrm{T}} d^{4}=f\left(x^{11}\right)+\left(c_{q}-\pi^{\mathrm{T}} a_{q}\right) \varepsilon
$$

Considerando que o índice $q$ foi escolhido tal que $c^{T} d^{q}<0$, então de (B.5) a direção $d^{q}$ é de descida.

De (B.4), podemos determinar o maior valor de $\varepsilon$, mantendo $x_{B} \geq \mathbf{0}$. $\Lambda$ partir dai, temos dois casos:

- Se $\alpha_{i q} \geq 0, i=1, \ldots, m$, então não há limitante para $\varepsilon, f(x) \rightarrow \infty$ (a direção $d^{q}$ é um raio de descida) e o problema (B.1) não tem solução ótima.

- Senão, para $i$ tal que $\alpha_{i q}<0$, tem-se:

$$
x_{B_{i}}^{0}+\varepsilon \alpha_{i q} \geq 0 \Rightarrow \varepsilon \leq \frac{x_{B_{i}}^{0}}{\alpha_{i q}},
$$

em que $x_{B_{1}}^{0}$ é a i-ésima componente de $x_{B}^{0}$.

Portanto, o maior valor para $\varepsilon$ é dado por:

$$
\varepsilon^{0}=\frac{x_{\beta_{p}}^{0}}{\alpha_{p q}}=\text { mínimo }\left\{\frac{x_{B_{i}}^{0}}{\alpha_{i q}} \mid \alpha_{i q}<0, i=1, \ldots, m\right\} \text { (critério da menor razão). (B.6) }
$$

Para esta escollha de $\varepsilon$, a $p$-ésima componente de $x_{B}$ se anula $\left(x_{B_{p}}=x_{B_{p}}^{0}-\frac{x_{B_{p}}^{0}}{\alpha_{p q}} \alpha_{p q}=0\right)$, enquanto que apenas uma variável de $x_{N}$ tornou-se positiva: $x_{q}=\varepsilon^{0} \geq 0$. Isto sugere uma nova partição básica, conforme o seguinte teorema. 
Teorema 4. Considere a nova partição $\boldsymbol{A}=\left[\boldsymbol{B}^{\prime}, N^{\prime}\right]$, em que a p-ésima coluna de $\boldsymbol{B}^{\prime}$ é dada por $a_{q} \in N$. A nova partição é básica factível, cuja solução básica associada é dada por: $x^{1}=x^{0}+\varepsilon^{0} d$.

De (B.5), podemos escolher qualquer $q$ tal que $c_{q}-\pi^{\mathrm{T}} \boldsymbol{a}_{q}<0$ para que um novo vértice $\left(x^{\mathrm{I}}\right)$ seja gerado com um valor menor para a função objetivo. Um critério (conhecido como a regra de Dantzig ou regra usual) para a escolha do índice $q$ da coluna que deverá tornar-se básica é dado por:

$$
c_{q}-\pi^{\mathrm{T}} \boldsymbol{a}_{q}=\operatorname{minimo}\left\{c_{j}-\pi^{\top} \boldsymbol{a}_{j}, j \in N\right\}
$$

Se $c_{q}-\pi^{\top} a_{q} \geq 0$ então, pelo teorema 3 , a solução básica factível ć uma solução ótima.

Desta forma, enquanto a condição de otimalidade do teorema 3 não for verificada, podemos repetir o procedimento descrito acima. Neste caso, os conjuntos de índices $B$ e $N$ são atualizados por:

$$
\begin{aligned}
& B \leftarrow(B \cup\{q\})-\{p\} \\
& N \leftarrow(N \cup\{p\})-\{q\}
\end{aligned}
$$

em que $B$ é o conjunto dos índices das colunas (ou variáveis) básicas. Tal procedimento descreve o Método Primal Simplex.

O método primal simplex gera, a partir de uma solução básica inicial factível, uma seqüência de soluçôes básicas factíveis do problema primal, convergente para uma solução ótima.

Caso uma solução básica factível não esteja disponivel para inicializar o método simplex, considere-se o seguinte problema artificial: (sem perda de generalidade, supomos $b_{i} \geq 0, i=1, \ldots, m$ ).

$$
\begin{aligned}
& \text { Minimizar } \quad f^{a}(\boldsymbol{x})=\sum_{i=n+1}^{n+m} x_{i} \\
& \text { Sujeito a }: \quad \sum_{j=1}^{n} a_{i j} x_{j}+x_{n+i}=b_{i} \quad i=1, \ldots, m \\
& x_{j} \geq 0, j=1, \ldots, n, \quad x_{n+i} \geq 0, i=1, \ldots, m
\end{aligned}
$$


Teorema 5. Seja $\left(x_{1}^{*}, \ldots, x_{n}^{*}, x_{n+1}^{*}, \ldots, x_{n+m}^{*}\right)$ uma solução ótima do problema artificial. Então se $\left(x_{1}^{*}, \ldots, x_{n}^{*}, x_{n+1}^{*}, \ldots, x_{n+m}^{*}\right)$ é uma solução factível para o programa original, se $\mathrm{c}$ somente se, $\left(x_{n+1}^{*}=\ldots=x_{n+n}^{*}=0\right)$.

O problcma artificial tem uma solução básica factível inicial óbvia (a matriz identidade, fornecida pelas colunas das variáveis artificiais), a partir da qual o algoritmo simplex inicia-se. Entretanto, nem sempre $x^{*}=\left(x_{1}^{*}, \ldots, x_{n+m}^{*}\right)$ é uma solução básica factível para o problema original. Isto acontece porque ao término da fase I, a base pode conter alguma variável artificial nula, digamos $x_{n+i}^{*}=0$, portanto uma degeneração. Com isto, a solução encontrada ao final da fase I, é factível (supondo $x_{n+i}^{*}=0, i=1, \ldots, m$ ), mas não é básica factível para o problema linear original. Ocorrências dessa natureza não impedem o uso da solução encontrada para gerar, a partir dela, uma solução básica factível para o problema original. Em geral, isto é feito pela substituição da variável artificial básica por outra variável não básica do problema original, com valor zero, por força da degencração. $\Lambda$ impossibilidade desta substituição significa que $i$-ésima restrição é combinação linear das demais e, portanto, pode ser eliminada do problema.

É dado a seguir o algoritmo primal simplex.

Algoritmo Primal Simplex

\section{Fase I}

Encontre uma partição básica primal-factível: $\boldsymbol{A}=(\boldsymbol{B}, N)$.

Faça $P A R E=F A L S O, I T=0$

\{A variável lógica P'ARE será falso até que a condição de otimalidade seja verificada e IT indica o número da iteração atual\}

\section{Fase II}

Enquanto PARE-FALSO faça:

Passo 1: Determine a solução básica primal factível: $\boldsymbol{x}_{B}=\boldsymbol{B}^{-1} \boldsymbol{b}$.

Passo 2: Teste a otimalidade

Passo 2.1: Determine a solução básica dual: $\pi^{\mathrm{T}}=\boldsymbol{c}_{B}^{\mathrm{T}} \boldsymbol{B}^{-1}$.

Passo 2.2: Determine o menor custo relativo:

$$
c_{q}-\pi^{\mathrm{T}} a_{q}=\text { mínimo }\left\{c_{j}-\pi^{\mathrm{T}} a_{j}, j \in N\right\} . \quad \text { / Regra de Dantzig } / /
$$


Passo 2.3: Se $c_{j}-\pi^{\mathrm{T}} a_{j} \geq 0 \quad \forall j \in N$, então a solução na itcração $T T$ é ótima.

\section{$P A R E=V E R D A D E$.}

Senão vá ao passo 3 .

Passo 3: Determine as coordenadas básicas da direção simplex: $\alpha_{q}=-\boldsymbol{B}^{-1} \boldsymbol{a}_{q}$

Passo 4: Se $\alpha_{i q} \geq 0, i=1, \ldots, m$, então o problema não tem solução ótima finita, o problema ć ilimitado. $P A R E=V E R D A D E$.

Senão

Determine o passo:

$$
\varepsilon^{0}=\frac{x_{B_{p}}^{0}}{\alpha_{p q}}=\text { minimo }\left\{\frac{x_{B_{i}}^{0}}{\alpha_{i q}} \mid \alpha_{i q}<0, i=1, \ldots, m\right\} .
$$

Passo 5: Atualize a partição básica: $a_{B} \leftrightarrow a_{q}, I T \leftarrow I T+1$.

\section{B.2. O Problema Dual}

Associado a cada problema de otimização lincar, existe um outro problema também linear chamado problema dual. Tipicamente usam-se duas formas (equivalentes) para definir o problema dual, dadas a seguir.

Suponha que o problema linear primal é dado na forma canônica:

$$
\begin{array}{ll}
\text { Minimizar } & c^{\mathrm{T}} \boldsymbol{x} \\
\text { Sujcito a: } & \boldsymbol{A} \boldsymbol{x} \geq \boldsymbol{b} \\
& \quad \boldsymbol{x} \geq \mathbf{0}
\end{array}
$$

então o problema linear dual é definido por:

$$
\begin{aligned}
& \text { Maximizar } \boldsymbol{b}^{\mathrm{T}} \boldsymbol{w} \\
& \text { Sujeito a: } \boldsymbol{A}^{\mathrm{T}} \boldsymbol{w} \leq \boldsymbol{c}
\end{aligned}
$$

$$
w \geq 0 .
$$

Há cxatamente uma variável dual para cada restrição primal e exatamente uma restrição dual para cada variável primal.

Outra definição equivalente da dualidade pode ser dada com o problema primal na seguinte forma padrão: 
Minimizar $c^{\mathrm{T}} \boldsymbol{x}$

Sujeito a: $\boldsymbol{A x}=\boldsymbol{b}$

$\boldsymbol{x} \geq \mathbf{0}$,

então o problema linear dual é definido por:

\section{Maximizar $\boldsymbol{b}^{\mathrm{T}} \boldsymbol{w}$ \\ Sujeito a: $\boldsymbol{A}^{\mathrm{T}} \boldsymbol{w} \leq \boldsymbol{c}$}

$w$ irrestrito de sinal.

\section{B.3. Relações Primal-Dual}

Considerando-se o para de problemas primal e dual (forma canônica ou padrão) pode-se mostrar uma série de relaçõcs entre os problemas que pode ser resumido no teorema fundamental da dualidade.

\section{Teorema 6. (Teorema fundamental da Dualidade)}

Considere o par de problemas (Pl) e (D l) (analogamente $\left(\mathrm{P}_{2}\right)$ e $\left(\mathrm{D}_{2}\right)$ ). Uma e somente uma das afirmativas seguintes é verdadeira.

a) Nenhum dos problemas tem solução factivel;

b) Um problema tem a função objetivo ilimitada, o outro problema associado é infactível;

c) $\Lambda$ mbos possucm soluções ótimas $x^{*}$ e $w^{*} \operatorname{com} c^{T} \boldsymbol{x}^{*}=w^{*} b$.

Apresentaremos agora, um importante teorema da teoria de dualidade, que é o teorema das folgas complementares, relacionando os problemas primal e dual.

Teorema 7. (Teorema das Folgas Complementares) Dado o par de problemas $\left(P_{1}\right)$ e $\left(D_{1}\right)$. Sejam $x^{*}$ e $w^{*}$ quaisquer soluções factíveis para $\left(\mathrm{P}_{1}\right)$ e $\left(\mathrm{D}_{1}\right)$ (analogamente $\left(\mathrm{P}_{2}\right)$ e $\left(\mathrm{D}_{2}\right)$ ) respectivamente, uma condição necessária e suficiente para que sejam soluções ótimas é que elas satisfaçam as relações abaixo: 


$$
\begin{aligned}
& \left(c_{j}-\boldsymbol{w}^{*} \boldsymbol{a}_{j}\right) x_{j}^{*}=0 \quad j=1, \ldots, n \\
& w_{i}^{*}\left(\boldsymbol{a}^{i} \boldsymbol{x}^{*}-b_{i}\right)=0 \quad i=1, \ldots, m .
\end{aligned}
$$

$\mathrm{Na}$ otimalidade se uma variável num problema é estritamente positiva, então a restrição correspondente no outro problema deve ser ativa, ou seja, ser satisfeita com igualdade. E se uma restrição num problema não é ativa, então a variável correspondente no outro problema deve ser zero. (No caso da forma padrão, a igualdade $w_{i}^{*}\left(\boldsymbol{a}^{i} \boldsymbol{x}^{*}-b_{i}\right)=0$ é sempre satisfeita, portanto redundante). 